\author{
Universidade de São Paulo \\ Instituto de Astronomia, Geofísica e Ciências Atmosféricas \\ Departamento de Astronomia
}

Fabio Cafardo

\title{
Exploring Sagittarius A* in gamma-rays with Fermi LAT Telescope
}

São Paulo 

Fabio Cafardo

\section{Exploring Sagittarius A* in gamma-rays with Fermi LAT Telescope}

Thesis presented to the Astronomy department of The Institute of Astronomy, Geophysics and Atmospheric Sciences at the University of São Paulo as partial requisite to obtain the $\mathrm{PhD}$ in Sciences title.

Concentration Area: Astronomy

Advisor: Prof. Dr. Rodrigo Nemmen

Versão Corrigida. O original encontra-se disponível na Unidade.

São Paulo 

to

[insert your name here] 



\section{Acknowledgements}

I have been helped by so many people my entire life. I take this moment to acknowledge how profoundly grateful I am.

To Julia, without whom this would be utterly impossible. Thank you very much for everything. Don't fret: fortunately, there is much more to come.

To my family for the support you gave me trough all my life.

To Flavia, who allowed for this adventure to start.

To all my friends, old and new, for the good moments. If you consider yourself a friend of mine, thank you!

To my advisor, Prof. Rodrigo, and the Black Hole Group: Prof. Roderik, Roberta, Raniere, Gustavo, Ivan, Artur, Maria Lucia, Douglas and Lucas. It was a pleasure working with you.

To all my teachers and professors, who helped to shape me into who I am.

To the Fermi-LAT Collaboration for providing an environment in which I could learn and get to know wonderful people.

To the people of São Paulo and Brazil for their sacrifice in funding the University of São Paulo, IAG and CNPq (who financed part of this PhD under grant number 142320/2016-1).

And to anyone that would like to see their name in this page. If you think you deserve my acknowledgment, here, take this one.

This thesis was written in $\mathrm{IAT}_{\mathrm{E}} \mathrm{X}$ using IAGTESE template. 

"An epigraph does more than set the tone for a poem-it raises the stakes before the poem even begins."

Heather Bowlan

"Eu não quero aprender mais nada, nunca mais, na minha vida."

@FalhamosBilu

"What?"

Richard M. Nixon

"The universe is a big place, perhaps the biggest."

Kilgore Trout 



\section{Resumo}

Sagitário A* — o buraco negro supermassivo no centro de nossa galáxia — já foi identificado na maior parte do espectro eletromagnético, de rádio a raios X. Em raios gama, se observa emissão difusa ao redor de Sagitário A* e uma fonte pontual foi detectada coincidindo com a posição do buraco negro supermassivo, mas ainda não há uma associação definitiva entre eles. Neste trabalho, usamos $\sim 11$ anos de observações da fonte pontual 4FGL J1745.6-2859 pelo Fermi Large Area Telescope para realizar análises detalhadas em quatro bandas de energia. Nossa meta é elucidar a origem da emissão de raios gama do centro da galáxia e investigar sua possível associação com o buraco negro supermassivo. Nós identificamos que os centróides das emissões se aproximam de Sagitário A* em energias mais altas e que eles estão associados espacialmente à distribuição de gás no centro da galáxia. Supondo que a fonte pontual de raios gama encontra-se no centro da galáxia, estimamos sua luminosidade em $2.61 \times 10^{36} \mathrm{erg} \mathrm{s}^{-1}$ no intervalo de energia entre $100 \mathrm{MeV}$ e $500 \mathrm{GeV}$. Este valor é consistente com a luminosidade bolométrica de Sagitário A*. Com base nas propriedades da fonte pontual, vários potenciais candidatos para esta emissão foram descartados, favorecendo uma interpretação em que os raios cósmicos são acelerados por - ou próximos de - Sagitário A*. Também elaboramos curvas de luz, com resolução temporal de 15 dias, em busca de variabilidade no fluxo de raios gama de 4FGL J1745.6-2859. Ao contrário do observado em comprimentos de onda mais longos, nós detectamos que a distribuição do seu fluxo de raios gama é compatível com uma Gaussiana, representativa de um processo aleatório normal, um indicativo de que o mecanismo de emissão de raios gama é distinto do regime de menores energias. Finalmente, a distribuição espectral de energia de 4FGL J1745.6-2859 apresenta um "pion-decay bump", característica de emissão de raios gama com origem hadrônica. Sua distribuição espectral 
de energia é, também, compatível com vários modelos hadrônicos para a emissão de raios gama de Sagitário A*. Nossos resultados indicam que a fonte pontual no centro da galáxia corresponde à contrapartida em raios gama de Sagitário $\mathrm{A} *$ em energias da ordem de GeV . As características desta emissão — sua coincidência espacial com reservatórios de gás, sua "energética", a falta de variabilidade e sua distribuição espectral de energia — sugerem que processos hadrônicos estejam por trás de sua origem. 


\section{Abstract}

Sagittarius A* $(\operatorname{Sgr} \mathrm{A} *)$ - the supermassive black hole $(\mathrm{SMBH})$ in the center of our galaxy - has been identified in most of the electromagnetic spectrum, from radio to $\mathrm{X}$ rays. Diffuse gamma-ray emission has been observed around Sgr A* and a gamma-ray point source has been detected coinciding with the SMBH's position, although there is still no definitive association between the two. In this work, we have used $\sim 11$ years of Fermi Large Area Telescope (LAT) observations of the point source 4FGL J1745.6-2859 and performed a detailed analysis across four energy bands. Our goal is to elucidate the nature of the gamma-ray emission at the Galactic Center (GC) and whether it is associated with the SMBH. We find that the centroids of the emission approach Sgr A*'s location as the energy increases and they are spatially associated with gas-rich regions in the GC. Assuming that the gamma-ray point source is located at the GC, we estimate a luminosity of $2.61 \times 10^{36} \mathrm{erg} \mathrm{s}^{-1}$ in the $100 \mathrm{MeV}$ to $500 \mathrm{GeV}$ energy range. This is consistent with Sgr A*'s bolometric luminosity. Based on the point source properties, we ruled out several potential candidates for its nature and favor a cosmic ray origin accelerated by - or in the vicinity of - the SMBH. We also created light curves (LCs), with time bins as short as 15 days, in search of variability in the 4FGL J1745.6-2859 gamma-ray flux. In contrast with Sgr A*'s flaring behavior in longer wavelengths, we detect that its gamma-ray flux distribution is compatible with a Gaussian, representative of a normal random process, hinting that the gamma-ray emission mechanism differs substantially from the low-energy regime. Finally, 4FGL J1745.6-2859's spectral energy distribution (SED) shows a "piondecay bump" characteristic of gamma-ray hadronic emission. Its SED is also compatible with several hadronic models for Sgr A*'s gamma-ray emission. Our results indicate that the point source at the GC is indeed the gamma-ray counterpart of $\mathrm{Sgr} \mathrm{A} *$ in the $\mathrm{GeV}$ 
range. The characteristics of this emission - its spatial coincidence with gas reservoirs, energetics, lack of variability and SED — suggest that hadronic processes are likely behind its origin. 


\section{List of Figures}

1.1 Multiwavelength observations of the inner few parsecs of the Galaxy . . . . 32

1.2 Cartoonistic depiction of the inner two parsecs of the Galaxy . . . . . . . 33

1.3 The Fermi gamma-ray sky . . . . . . . . . . . . . . . . . . . . . 34

1.4 The S-star cluster . . . . . . . . . . . . . . . . . . . . . . 38

1.5 Twenty six years observations of S2 star orbiting Sgr A* . . . . . . . . 39

1.6 Sgr A*'s broad band spectrum . . . . . . . . . . . . . . . 46

1.7 Two bright X-rays flares from Sgr A* fit with a hotspot model . . . . . . . 48

1.8 The Fermi Bubbles . . . . . . . . . . . . . . . . . . . . . . . . . . . . . . 52

1.9 Sensitivity of different gamma-ray detectors . . . . . . . . . . 55

1.10 Earth's atmospheric opacity . . . . . . . . . . . . . . . . . 59

1.11 The Fermi Gamma-ray Space Telescope . . . . . . . . . . . . . . . . . . 60

1.12 The Fermi-LAT instrument and its constituents . . . . . . . . . . . . 63

1.13 Fermi-LAT's effective area as function of energy and incidence angle . . . . 67

1.14 Fermi-LAT's PSF as function of energy and incidence angle . . . . . . . 69

1.15 Fermi-LAT's systematic uncertainty on the effective area . . . . . . . . . 70

1.16 Fermi-LAT's PSF systematic uncertainty . . . . . . . . . . . . 71

1.17 Spatial distribution and classification of 4FGL sources . . . . . . . . . 74

1.18 Central source's spectral model in 4FGL . . . . . . . . . . . . . . . 78

2.1 Energy bands used in the analysis . . . . . . . . . . . . . . 84

2.2 Residuals distribution examples for the low energy model . . . . . . . . . 100

2.3 Residuals map of the Universal Model . . . . . . . . . . . . . . . . . . . . 102

2.4 Residuals maps in the four tighter energy bands . . . . . . . . . . . . . . . 103

2.5 TS map of the Universal Model . . . . . . . . . . . . . . . . . . . . 105 
2.6 TS maps of the four tighter energy bands . . . . . . . . . . . . . . 106

2.7 TS map of the Universal Model after removing the central source . . . . . 107

2.8 TS maps of the four energy bands after removing the central source . . . . 108

2.915 days energy flux LC of 4FGL J1747.2-2957 . . . . . . . . . . . . 115

2.10 Statistical uncertainties distribution of 4FGL J1747.2-2957 15 days energy flux LC . . . . . . . . . . . . . . . . . . . 116

2.11 Relative dispersion distribution of 4FGL J1747.2-2957 15 days energy flux

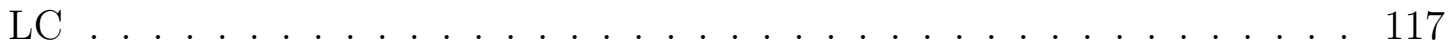

2.1215 days bins LCs of 4FGL J1745.6-2859 . . . . . . . . . . . . . . . . . 119

3.1 Best-fit spectral models . . . . . . . . . . . . . . . . . . . 126

3.2 Central source position as a function of energy . . . . . . . . . . . . 128

3.3 Distance between the central source and $\operatorname{Sgr} A *$ as a function of energy . . 129

3.4 UL on the spatial extension of the central source as a function of energy . . 130

3.5 Distributions of the error radii in the different Fermi Catalogues . . . . . . 131

3.615 days LCs of 4FGL J1745.6-2859 versus average flux . . . . . . . . . . 132

3.715 days LCs of 4FGL J1745.6-2859 versus 5 bins rolling average flux . . . 133

3.8 The flux distribution of the 15 days bins LCs . . . . . . . . . . . . . . . . 135

3.915 days LCs of 4FGL J1745.6-2859 fitted with linear functions . . . . . 136

3.1090 days LCs of 4FGL J1745.6-2859 versus average flux in the $300 \mathrm{MeV}-3$ GeV energy band . . . . . . . . . . . . . . . . . . . . 137

3.1190 days LCs of 4FGL J1745.6-2859 versus average flux in the 3-10 GeV energy band . . . . . . . . . . . . . . . . . . . . . . . 138

3.12180 days LCs of 4FGL J1745.6-2859 versus average flux in the 10-500 GeV energy band . . . . . . . . . . . . . . . . . . . . . 139

3.13 90 days LCs of 4FGL J1745.6-2859 versus 5 bins rolling average flux in the $300 \mathrm{MeV}-3 \mathrm{GeV}$ energy band . . . . . . . . . . . . . . . . . 140

3.1490 days LCs of 4FGL J1745.6-2859 versus 5 bins rolling average flux in the 3-10 GeV energy band . . . . . . . . . . . . . . . . . . . . . 141

3.15180 days LCs of 4FGL J1745.6-2859 versus 5 bins rolling average flux in the $10-500 \mathrm{GeV}$ energy band . . . . . . . . . . . . . . . . . . 142

3.16 Linear fit to the 90 days bins LCs in the $300 \mathrm{MeV}-3 \mathrm{GeV}$ energy band . . 143 
3.17 Linear fit to the 90 days bins LCs in the 3-10 GeV energy band . . . . . . 144

3.18 Linear fit to the 180 days bins LCs in the 10-500 GeV energy band . . . . 145

3.19 The resulting SED for the Universal Model . . . . . . . . . . . . . . . . . . 147

4.1 Source position and gas distribution in the GC . . . . . . . . . . . 151

4.2 The 4FGL blazars energy flux distribution . . . . . . . . . . . . . 156

4.3 Trap et al. (2011) observations of a NIR and flare from $\operatorname{Sgr} \mathrm{A} *$. . . . . . 160

4.415 days energy flux LC of 4FGL J1745.6-2859 versus remarkable events . 162

4.5 15 days photon flux LC of 4FGL J1745.6-2859 versus remarkable events . 163

4.6 A simple model for the different energy bands emission . . . . . . . . . . 164

4.7 The combination of the narrower energy bands energy flux LCs . . . . . . 165

4.8 The combination of the narrower energy bands photon flux LCs . . . . . 166

4.9 4FGL J1745.6-2859 SED compared with different models for Sgr A*'s emis-

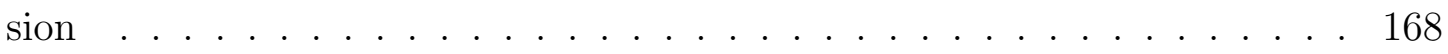

D.1 Examples of discontinuous models not used in this work . . . . . . . . . 232

E.1 Residual maps for previous analyses for the 3 highest bands . . . . . . . 234

E.2 TS maps with the source for previous analyses for the 3 highest bands . . . 235

E.3 TS maps without the source for previous analyses for the 3 highest bands . 236

G.1 Event types tests for the 60-300 MeV interval: central source's position . . 242

H.1 Residuals distribution for the Universal Model offspring . . . . . . . . . . . 246

I.1 15 days photon flux LC of 4FGL J1747.2-2957 . . . . . . . . . . 248

I.2 Statistical uncertainties distribution of 4FGL J1747.2-2957 15 days photon flux LC . . . . . . . . . . . . . . . . . . . 249

I.3 Relative dispersion distribution of 4FGL J1747.2-2957 15 days photon flux

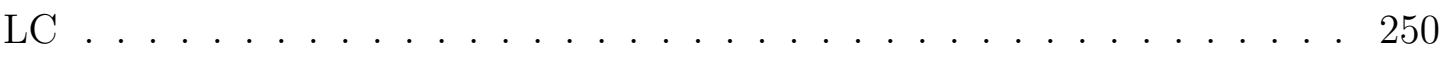

J.1 45 days energy flux LC of 4FGL J1747.2-2957 in the $100 \mathrm{MeV}-500 \mathrm{GeV}$ energy range . . . . . . . . . . . . . . . . . 252

J.2 Statistical uncertainties distribution of 4FGL J1747.2-2957 45 days energy flux LC in the $100 \mathrm{MeV}-500 \mathrm{GeV}$ energy range . . . . . . . . . . . 252 
J.3 Relative dispersion distribution of 4FGL J1747.2-2957 45 days energy flux LC in the $100 \mathrm{MeV}-500 \mathrm{GeV}$ energy range . . . . . . . . . . . . . 253

J.4 45 days photon flux LC of 4FGL J1747.2-2957 in the $100 \mathrm{MeV}-500 \mathrm{GeV}$ energy range . . . . . . . . . . . . . . . . . . 2 253

J.5 Statistical uncertainties distribution of 4FGL J1747.2-2957 45 days photon flux LC in the $100 \mathrm{MeV}-500 \mathrm{GeV}$ energy range . . . . . . . . . . 254

J.6 Relative dispersion distribution of 4FGL J1747.2-2957 45 days photon flux LC in the $100 \mathrm{MeV}-500 \mathrm{GeV}$ energy range . . . . . . . . . . . . . 254

J.7 90 days energy flux LC of 4FGL J1747.2-2957 in the $300 \mathrm{MeV}-3 \mathrm{GeV}$ energy range . . . . . . . . . . . . . . . . . . . 255

J.8 Statistical uncertainties distribution of 4FGL J1747.2-2957 90 days energy flux LC in the $300 \mathrm{MeV}-3 \mathrm{GeV}$ energy range . . . . . . . . . . . . . . . 256

J.9 Relative dispersion distribution of 4FGL J1747.2-2957 90 days energy flux LC in the $300 \mathrm{MeV}-3 \mathrm{GeV}$ energy range . . . . . . . . . . . . 256

J.10 90 days photon flux LC of 4FGL J1747.2-2957 in the 300 MeV-3 GeV energy

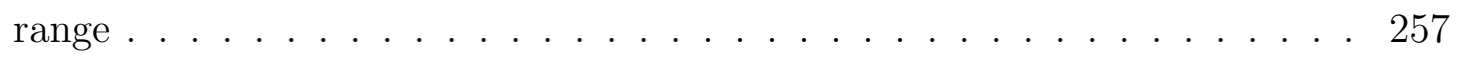

J.11 Statistical uncertainties distribution of 4FGL J1747.2-2957 90 days photon flux LC in the $300 \mathrm{MeV}-3 \mathrm{GeV}$ energy range . . . . . . . . . . . . . 257

J.12 Relative dispersion distribution of 4FGL J1747.2-2957 90 days photon flux LC in the $300 \mathrm{MeV}-3 \mathrm{GeV}$ energy range . . . . . . . . . . . 258

J.13 90 days energy flux LC of 4FGL J1747.2-2957 in the 3-10 GeV energy range 259

J.14 Statistical uncertainties distribution of 4FGL J1747.2-2957 90 days energy flux LC in the $3-10 \mathrm{GeV}$ energy range . . . . . . . . . . . . 259

J.15 Relative dispersion distribution of 4FGL J1747.2-2957 90 days energy flux LC in the $3-10 \mathrm{GeV}$ energy range . . . . . . . . . . . . . . . . 260

J.16 90 days photon flux LC of 4FGL J1747.2-2957 in the 3-10 GeV energy range260

J.17 Statistical uncertainties distribution of 4FGL J1747.2-2957 90 days photon flux LC in the $3-10 \mathrm{GeV}$ energy range . . . . . . . . . . . . . . . 261

J.18 Relative dispersion distribution of 4FGL J1747.2-2957 90 days photon flux LC in the $3-10 \mathrm{GeV}$ energy range . . . . . . . . . . . . . . 261

K.1 45 days LCs of 4FGL J1745.6-2859 versus average flux . . . . . . . . . . 264 
K.2 45 days LCs of 4FGL J1745.6-2859 versus 5 bins rolling average flux . . . 265

K.3 The flux distribution of the 45 days bins LCs . . . . . . . . . . . . . 266

K.4 45 days LCs of 4FGL J1745.6-2859 fitted with a linear function . . . . . . 268

L.1 Central point position as a function of energy . . . . . . . . . . 270

M.1 TS for the 15 days LC of 4FGL J1745.6-2859 (100 MeV-500 GeV energy

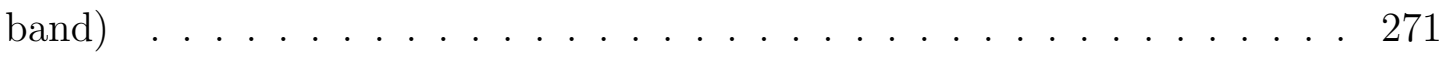

M.2 TS for the 90 days LC of 4FGL J1745.6-2859 (300 MeV-3 GeV energy band)272

M.3 TS for the 90 days LC of 4FGL J1745.6-2859 (3-10 GeV energy band) . . 272

M.4 TS for the 180 days LC of 4FGL J1745.6-2859 (10-500 GeV energy band) 273

M.5 TS for the 45 days LC of 4FGL J1745.6-2859 (100 MeV-500 GeV energy

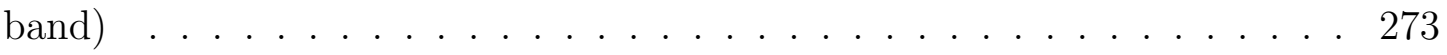





\section{List of Tables}

1.1 Accretion rate as a function of distance from $\operatorname{Sgr} A * \ldots . . . . . .42$

1.2 Central source positions in Fermi-LAT's catalogs . . . . . . . . . . 777

2.1 Systematic uncertainties of the light curves used in this work . . . . . . . 117

3.1 Central source gamma-ray flux . . . . . . . . . . . . . . . . . 127

3.2 Results of the Shapiro-Wilk and the D'Agostino's $\mathrm{K}^{2}$ normality tests for the 15 days $\mathrm{LCs}$. . . . . . . . . . . . . . . . . . . . . . . 134

3.3 Results of the Anderson-Darling normality test for the 15 days LCs . . . . 134

3.4 Results of a linear fit to the 15 days bins LCs data . . . . . . . . . . 136

3.5 Results of linear fits to the for the narrower energy bands LCs . . . . . . . 145

C.1 New sources found in the $100 \mathrm{MeV}$ to $500 \mathrm{GeV}$ energy range. . . . . . . . . 230

E.1 Central source gamma-ray flux in previous analyses . . . . . . . . . . 237

F.1 New sources found in the 60 to $300 \mathrm{MeV}$ energy range. . . . . . . . . . . 239

G.1 Event types tests for the 60-300 MeV interval: central source's flux . . . . 243

K.1 Results of the Shapiro-Wilk and the D'Agostino's K² normality tests for the 45 days $\mathrm{LCs}$. . . . . . . . . . . . . . . . . . . . . . . . 267

K.2 Results of the Anderson-Darling normality test for the 45 days LCs . . . . 267

K.3 Results of a linear fit to the 45 days bins LCs data . . . . . . . . . . 268 



\section{Abbreviations}

- 1FGL: First Fermi-LAT Catalog

- 1FLE: First Fermi Low Energy Catalog

- 2D: Two-dimensional

- 2FGL: Second Fermi-LAT Catalog

- 3D: Three-dimensional

- 3FGL: Third Fermi-LAT Catalog

- 4FGL: Fourth Fermi-LAT Catalog

- 4FGL-DR2: Fermi-LAT 10-year Catalog

- AIC: Akaike Information Criterion

- AGN: Ative Galactic Nuclei

- BH: Black Hole

- CND: Circum-Nuclear Disk

- CR: Cosmic Rays

- CTA: Cherenkov Telescope Array

- DM: Dark Matter

- EAS: Extensive Air Showers 
- EHT: Event Horizon Telescope

- ELT: Extremely Large Telescope

- GC: Galactic Center

- Gamma-ray Bursts: GRB

- GRMHD: General Relativistic Magneto Hydrodynamics

- GW: Gravitational Waves

- H.E.S.S.: High Energy Stereoscopic System

- IACT: Imaging Air Cherenkov Technique

- IC: Inverse Compton

- IRFs: Instrument Response Functions

- ISCO: Innermost Stable Circular Orbit

- LAT: Large Area Telescope

- LC: Light Curve

- GTI: Good Time Intervals

- MCMC: Markov chain Monte Carlo

- NIR: Near Infrared

- $N_{\text {Pred }}$ : Predicted Number of Counts

- PWN: Pulsar Wind Nebulae

- PSF: Point Spread Function

- RIAF: Radiatively Inefficient Accretion Flow

- RoI: Region of Interest

- Sgr A*: Sagittarius A* 
- SED: Spectral Energy Distribution

- SMBH: Supermassive Black Hole

- SNR: Supernova Remnants

- SSC: Synchrotron Self Compton

- UL: Upper Limit

- VLT: Very Large Telescope

- WIMP: Weakly Interacting Massive Particle 



\section{Contents}

1. Introduction . . . . . . . . . . . . . . . . . . 29

1.1 The motivations and goals of this thesis . . . . . . . . . . . 29

1.2 The Galactic Center . . . . . . . . . . . . . . . . . . . . . 30

1.2.1 The Galactic Center in gamma-rays . . . . . . . . . . . . 34

1.3 Sagittarius A $\ldots \ldots \ldots \ldots \ldots \ldots \ldots$

1.3.1 A historical perspective . . . . . . . . . . 36

1.3.2 The Galactic Center black hole . . . . . . . . . . . . . . . 37

1.3.2.1 Basic facts about $\operatorname{Sgr} \mathrm{A} * \ldots \ldots \ldots$

1.3.3 Multiwavelength emission ................. 42

1.3.3.1 Sagittarius A* in gamma-rays . . . . . . . . . . . 43

1.3.3.2 The two states of Sagittarius A* . . . . . . . 45

1.3.3.3 Variability and flares . . . . . . . . . . 47

1.3.3.4 Hints of past activity of Sagittarius A* emission . . . . . 51

1.3.4 Models for Sagittarius A* emission . . . . . . . . . . . 52

1.4 Other gamma-ray telescopes . . . . . . . . . . . . . . 54

1.4.1 Space-based instruments . . . . . . . . . . . . . 555

1.4.2 Ground-based Cherenkov telescopes . . . . . . . . . . . 56

1.4.2.1 The Imaging Air Cherenkov Technique . . . . . . . . . . . 57

1.4.2.2 The Extensive Air Showers Technique . . . . . . . . 58

1.5 Fermi Gamma-ray Space Telescope . . . . . . . . . . . . . . . . . . 58

1.5.1 The Fermi Large Area Telescope . . . . . . . . . . . . . . . . . . . 61

1.5.2 Fermi-LAT data products . . . . . . . . . . . . 64 64

1.5.3 Fermi-LAT performance . . . . . . . . . . . . . 66 
1.5.4 Caveats and systematics . . . . . . . . . . . . 68

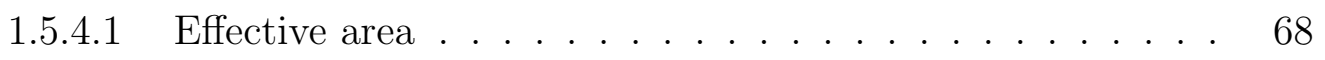

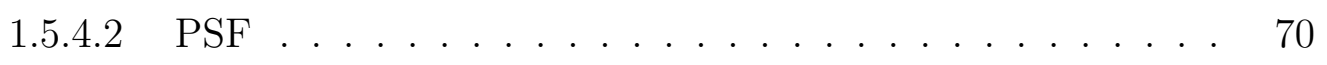

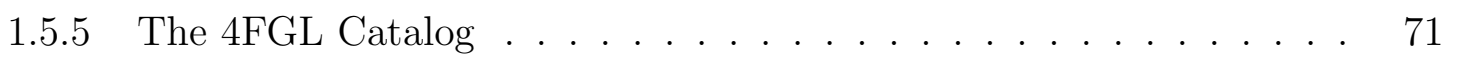

1.5.5.1 The diffuse models . . . . . . . . . . . . . 75

1.5.5.2 The 4FGL J1745.6-2859 source . . . . . . . . . . . 77

1.6 The production of astrophysical gamma-rays . . . . . . . . . 78

1.6.1 Leptonic emission . . . . . . . . . . . . . . . . . . . . . 79

1.6.2 Hadronic emission . . . . . . . . . . . . . . . . 79

1.6.3 Dark matter self annihilation . . . . . . . . . . . 80

1.7 Publication resulting from this work . . . . . . . . . . . . . . 80

2. Data and Methods .................... 83

2.1 Data . . . . . . . . . . . . . . . . . 83

2.1.1 Data selection: Universal Model . . . . . . . . . . . . . 8 85

2.1.2 Data selection: low energy custom model . . . . . . . . . . 8 86

2.1 .3 Energy boundaries . . . . . . . . . . . . . . . . 87

2.2 Maximum likelihood modeling . . . . . . . . . . . . . . 87

2.2 .1 The likelihood function . . . . . . . . . . . . . . 88

2.2.1.1 Likelihood model fitting with Fermitools . . . . . . . . 90

2.2.1.2 Model selection . . . . . . . . . . . . . . . . . . . . 91

2.3 Defining models with the Fermitools _ . . . . . . . . . . . . 92

2.3.1 Universal Model . . . . . . . . . . . . . . . . . . . . . . . . . . . . 93

2.3.2 Custom model $(60-300 \mathrm{MeV}) \quad \ldots \ldots \ldots$

2.3.3 Evaluating the quality of the fitting process $\ldots \ldots \ldots$

2.4 Assessing the source position . . . . . . . . . . . . . . . . 109

2.4.1 Identifying the source position with the Fermitools . . . . . . . 109

2.4 .2 Systematic uncertainties . . . . . . . . . . . . . 111

2.5 Variability of the GC . . . . . . . . . . . . 112

2.5 .1 Creating a light curve $\ldots \ldots \ldots \ldots \ldots \ldots$

2.5.2 Systematic uncertainties . . . . . . . . . . . . . . 114

2.5.3 Searching for variability in the LCs . . . . . . . . . . . 118 
2.6 Spectral Energy Distribution . . . . . . . . . . . . . . . . . . 122

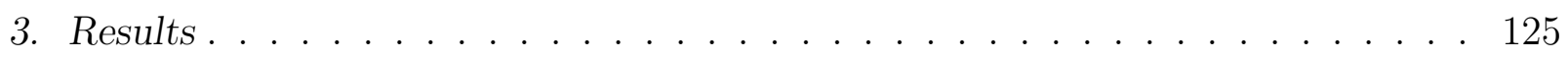

3.1 Spectral model . . . . . . . . . . . . . . . . . . . . . . 125

3.2 Position in different energy bands . . . . . . . . . . . . . . . . . . 127

3.3 Light curves . . . . . . . . . . . . . . . . . . . . . 131

3.3 .115 days bins, Universal Model . . . . . . . . . . . . . 132

3.3.2 Separate energy intervals . . . . . . . . . . . . . 137

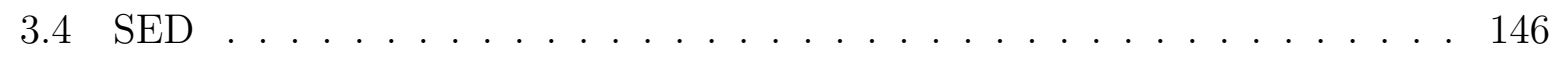

4. Discussion . . . . . . . . . . . . . . . . . . . . . . . 149

4.1 Energetics and centroid . . . . . . . . . . . . . 149

4.1.1 Nature of emission . . . . . . . . . . . . . . . . 151

4.2 On the temporal flux evolution . . . . . . . . . . . . 157

4.2.1 The 15 days bins LC . . . . . . . . . . . . . . . . . 157

4.2.2 The LCs created for the narrower energy bands . . . . . . . . 163

4.3 SED and emission models . . . . . . . . . . . . . . . 167

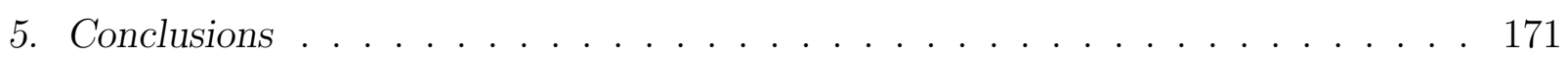

5.1 The evidence points to Sagittarius A . . . . . . . . . . 171

5.2 Future perspectives . . . . . . . . . . . . . . . 173

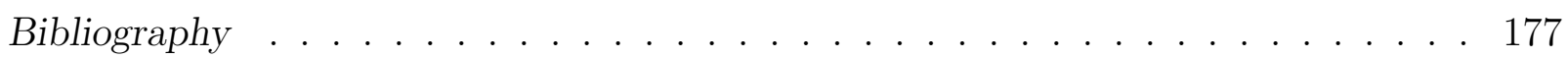

Appendix 201

A. Article: Fermi LAT observations of Sagittarius A*: I - Imaging Analysis . . . . 203

B. Additional Publications . . . . . . . . . . . . . . . . . . 223

C. New sources found in the analysis between $100 \mathrm{MeV}$ and $500 \mathrm{GeV}$. . . . . . 229

D. Examples of discontinuous models . . . . . . . . . . . . . . . . . . 231

E. Comparing the results obtained through the minimal fitting method to a more standard approach . . . . . . . . . . . . . . . . . . 233 
F. New sources found in the analysis between 60 and $300 \mathrm{MeV}$. . . . . . . . 239

G. Comparing different event type selection for the low energy model . . . . . . . . 241

H. Residuals distribution for the Universal Model offspring . . . . . . . . . . . . . . 245

I. Estimating the systematic uncertainties in the 15 days photon flux LC . . . . 247

J. Estimating the systematic uncertainties in the LCs . . . . . . . . . . . . . . . 251

J.1 Systematic uncertainties in the 45 days LC . . . . . . . . . . . . . . . . 251

J.1.1 Energy flux . . . . . . . . . . . . . . . . 252

J.1.2 Photon flux . . . . . . . . . . . . . . . . . . . 253

J.2 Systematic uncertainties in the 90 days, $300 \mathrm{MeV}-3 \mathrm{GeV}$ LC . . . . . . . 255

J.2.1 Energy flux . . . . . . . . . . . . . . . 255

J.2.2 Photon flux . . . . . . . . . . . . . . . . . . . . . . . . . . . . 257

J.3 Systematic uncertainties in the 90 days, 3-10 GeV LC . . . . . . . . . . . 258

J.3.1 Energy flux . . . . . . . . . . . . . . . . . 259

J.3.2 Photon flux . . . . . . . . . . . . . . . . . . . . . 260

K. The 45 days bins LC created based on the Universal Model (100 MeV-500 GeV) 263

L. The $95 \%$ confidence level central point position as a function of energy . . . . . 269

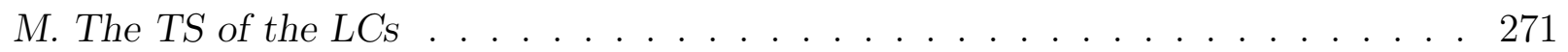


Chapter 1

\section{Introduction}

In 1915, Albert Einstein published the theory of general relativity, forever changing the way we understand gravity. Only a few months later, Karl Schwarzschild found a solution to the Einstein field equations that would characterize a black hole $(\mathrm{BH})$ as a region of space from which nothing can escape. The 1960's and 1970's are brimful of theoretical works that developed and solidified the concept of BHs in the science literature and, also, in the zeitgeist.

More recently, the interest in these objects was propelled by extraordinary observational results, such as the confirmation of the presence of a SMBH in the center of Our Galaxy, Sgr A*, inferred from stellar orbits; the detection of gravitational waves from the merger of two black holes in 2015; and the first image of a BH silhouette captured by the Event Horizon Telescope (EHT) in 2019. Two of these exceptional results were rightfully awarded with Nobel prizes: in 2017, Rainer Weiss, Kip S. Thorne and Barry C. Barish shared the Nobel Prize in Physics for the detection of gravitational waves and in 2020, Andrea Ghez and Reinhard Genzel were awarded the Nobel Prize in Physics for the showing that Sgr $\mathrm{A} *$ contains a $\mathrm{SMBH}$.

This thesis is the result of over 4 years of work to provide our small contribution to the scientific effort of understanding these alluring objects.

\subsection{The motivations and goals of this thesis}

Sgr $A *$ is a interesting laboratory to investigate the behavior and environment of SMBHs in the center of galaxies. Although it has been observed in most of the electro- 
magnetic spectrum, there is still no definitive association between the gamma-ray emission from the Galactic Center (GC) and Sgr A*.

The Fermi-LAT source 4FGL J1745.6-2859 is coincident with Sgr A* position and hence an appealing subject to our research of Sgr A* gamma-ray emission. We focused our work in this source, using $\sim 11$ years observations with Fermi-LAT in the $60 \mathrm{MeV}$ to $500 \mathrm{GeV}$ energy range, with the aim of elucidating the physical nature of the high energy processes associated with the source and investigating the relation of the gammaray emission with Sgr A*. Therefore, we characterized its image, position and extension; studied its flux variability, searching for flaring events; and measured the SED.

\subsection{The Galactic Center}

The GC is an extraordinary laboratory to investigate the physical processes happening in the vicinity of a SMBH. Since it is at least 5 orders of magnitude closer than the nearest quasar the GC is subject to high-resolution observations, with much more detail than possible in any other galaxy nucleus.

It is believed that every sufficiently massive galaxy harbors a SMBH in its center (Lynden-Bell, 1969; Kormendy and Richstone, 1995; Miyoshi et al., 1995; Heckman and Best, 2014). Including the Mily Way, these objects dominate the dynamics and hence much of the physical processes, occurring in the innermost regions of the nuclei. In this thesis, we focus on the GC's SMBH, Sgr A*, behavior in gamma-rays with Fermi-LAT observations. But, before we delve in Sgr A* (described in Section 1.3), we will present a brief review of the GC picture. The focus is on the central few parsecs of the Galaxy, which contain a dense star cluster, several gaseous components and the SMBH.

The central $1.5 \mathrm{pc}$ is a mostly ionized, low density "cavity". It is pervaded by Sagittarius A West (also referred as the mini-spiral), a region of H II composed of filaments with the appearance of a three-arm spiral, from the point of view of the Earth Lo and Claussen, 1983; Christopher et al., 2005); and a concentration of extremely hot gas, detected in Xrays (Baganoff et al., 2001, 2003, Muno et al., 2004). At $\sim 1.5$ pc from the GC there is a sharp transition between the central cavity and the circumnuclear disk (CND). The CND has a $\sim 1.5-4$ pc radius and is comprised of orbiting dense molecular clouds and warm 
dust (Becklin et al., 1982; Guesten et al., 1987, Jackson et al., 1993, Christopher et al., 2005; Armillotta et al., 2019). In Figure 1.1, adapted from Genzel et al. (2010), we show multiwavelength observations of the central 3 pc of our Galaxy. The structures mentioned are highlighted in the right panels, along with other features.

Not only gas inhabits the CND. A dense star cluster is also found in the inner parts of the Galaxy. The stellar density inside this cluster increases inward, from a scale of tens of parsecs to within the GC (Becklin and Neugebauer, 1968; Genzel et al., 1994, Do et al., 2013; Calderon et al., 2020). Many of these are young massive stars (Genzel et al., 1996; Blum et al., 1997; Paumard et al., 2003, 2006; Tanner et al., 2006). In the inner 1" of the Galaxy, there is a concentration of mainly B stars: the S-star cluster, discussed in more details in Section 1.3.2 (see, also, Figure 1.4) (Schödel et al., 2009; Habibi et al., 2017). The nuclear star cluster is one of the highest concentrations of massive young stars in the Galaxy. The presence of such young stars in the extreme environment so close to a SMBH offers a challenge for the current understanding of star formation and the dynamics close to a SMBH.

Inside its sphere of influence - a region around a SMBH in which its gravitational potential dominates (see Section 1.3.2.1), in the case of Sgr A* it is delimited by a radius of 2-3 pc (Genzel et al., 2010; Eckart et al., 2017) —Sgr A* dominates the mass distribution. Additional contribution arises from stars, stellar remnants (stellar mass BHs and neutron stars) (Freitag et al., 2006; Ghez et al., 2008; Gillessen et al., 2009; Gillessen et al., 2009), gas (Christopher et al., 2005) and dark matter (Navarro et al., 1997; Vasiliev and Zelnikov, 2008) though the later two components do not contribute significantly. Figure 1.2, obtained from Murchikova et al. (2019), shows a cartoonistic depiction of the region inside the CND. Other than the structures already described in this Section, they show the orbits of the $\mathrm{S} 2$ star and the G2 object (both described in Section 1.3.2); a $10^{4} \mathrm{~K}$ ionized gas disk within $2 \times 10^{4} R_{S}$ detected by the authors using the $1.3 \mathrm{~mm}$ recombination line $\mathrm{H} 30 \alpha$ (that they interpret in terms of a rotating disk of mass $10^{-4}$ to $10^{-5} M_{\odot}$ ), the clockwise stellar system (a concentration of stars, inside the nuclear star cluster, arranged in a Keplerian disk around Sgr A* Genzel et al. 2000) and the Keplerian fall-off radius (the radius of 0.3 pc at which the point mass of Sgr A* visibly dominates the velocity dispersion of the stars in the surrounding cluster and the contribution of an extended component, related to the stars in the nuclear star cluster, is negligible, Schödel et al. 2009). 


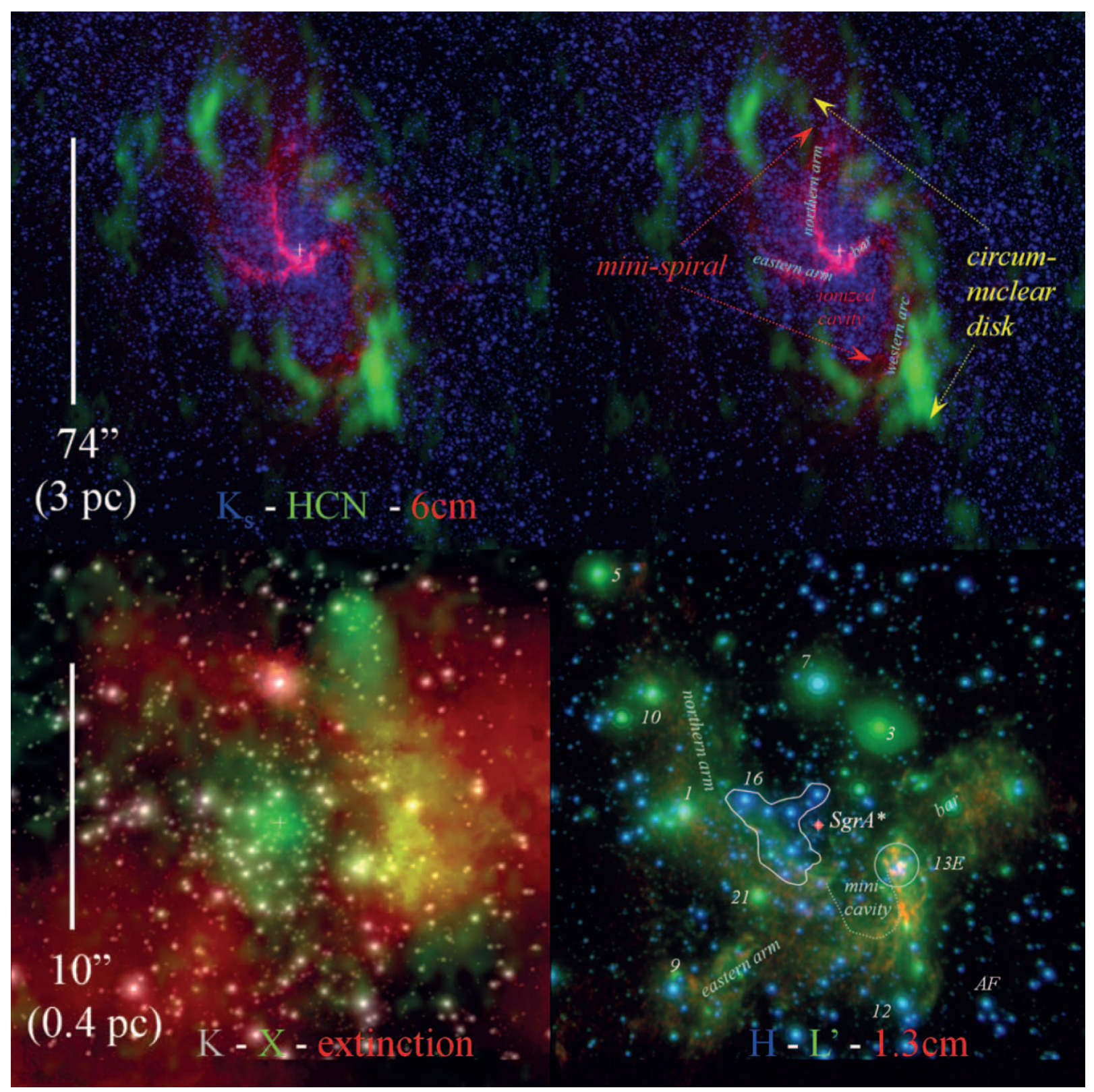

Figure 1.1: The central few parsecs of the Galaxy. Top row: multiwavelength overview of the central parsecs. Top left panel: $6 \mathrm{~cm}$ emission (pink: Yusef-Zadeh et al. 1986, Roberts and Goss 1993), HCN 1-0 emission (green: Christopher et al. 2005), and NIR K-band (blue: Schödel et al. 2007). Top right panel: the same as the top left panel, with interstellar features marked. Bottom row: zoom into the central region. Bottom left panel: K-band (white: Genzel et al.|2003), derived dust extinction (red-yellow: Schödel et al. 2007), and x-ray emission (green: Baganoff et al. 2003). Bottom right: NIR adaptive optics H-band and L'-band (blue and green, respectively: Genzel et al. 2003), and $1.3 \mathrm{~cm}$ radio continuum (red: Zhao and Goss 1998), some of the interstellar features are marked. Sgr A*'s position is marked with a faint white cross in every panel. North is up and East is to the left. The galactic plane runs at a position angle of $32^{\circ}$ southwest-northeast. The lengths in pc correspond to structures at the GC distance. Source: adapted from Genzel et al. (2010). 


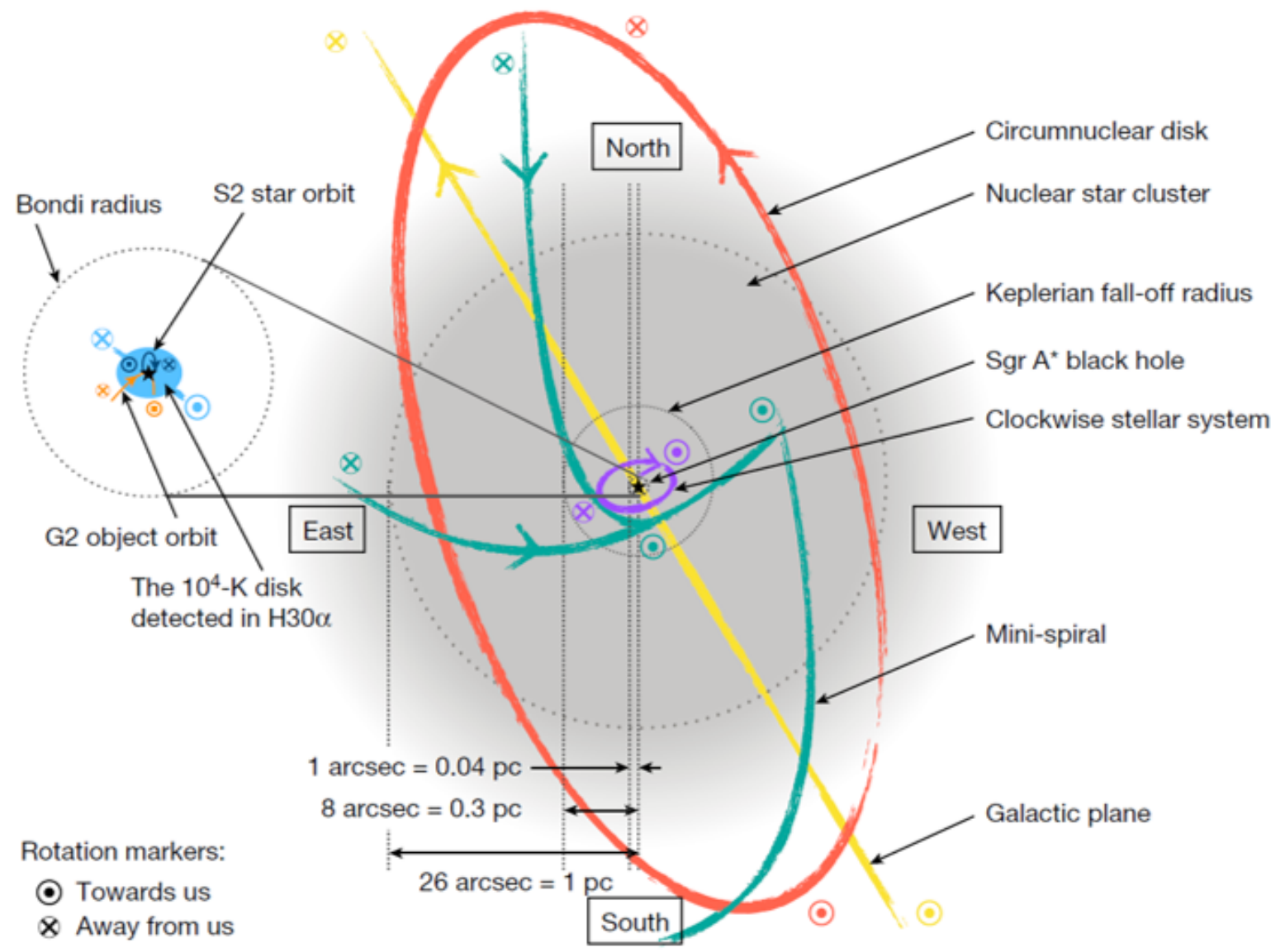

Figure 1.2: Schematic plot, to scale, of the inner two parsecs of the Galaxy. The main structures are indicated by different colors. The structures within the $R_{B}$ are plotted in the zoomed-in region on the left. The direction of rotation of the structures are shown with arrows, the circled cross indicates recession from Earth, and the circled dot indicates approach to Earth. The projected distances in parsecs were calculated based on the GC distance (at this distance, $1 " \approx 0.04 \mathrm{pc}$ ). Source: Murchikova et al. $(2019)$. 
Beyond the East edge of the CND, lies Sagittarius A East, a young supernova remnant. Also, the region is surrounded by several massive and dense molecular clouds with scale of 5-100 pc. (Gusten and Downes, 1980; Mezger et al., 1996).

Despite the wealthy reservoir of gas in its surroundings, $\operatorname{Sgr} A *$ is remarkably faint. This is the result of a combination of low efficiency converting the accreting material into radiative energy and a decrease of the accretion rate toward the SMBH (see Section 1.3.4).

\subsubsection{The Galactic Center in gamma-rays}

The GC is the brightest region of the gamma-ray sky. In Figure 1.3 we show 5 years of Fermi-LAT observations of the whole sky in energies $>1 \mathrm{GeV}$. Since the image is in Galactic coordinates, the GC is located at its center. The most prominent feature is the bright band of diffuse glow along the map's center, which marks the central plane of the Galaxy. This is the result of cosmic rays (CR) collisions with the interstellar gas (more details in Sections 1.5.5.1 and 1.6). The GC is embedded in this gamma-ray component.

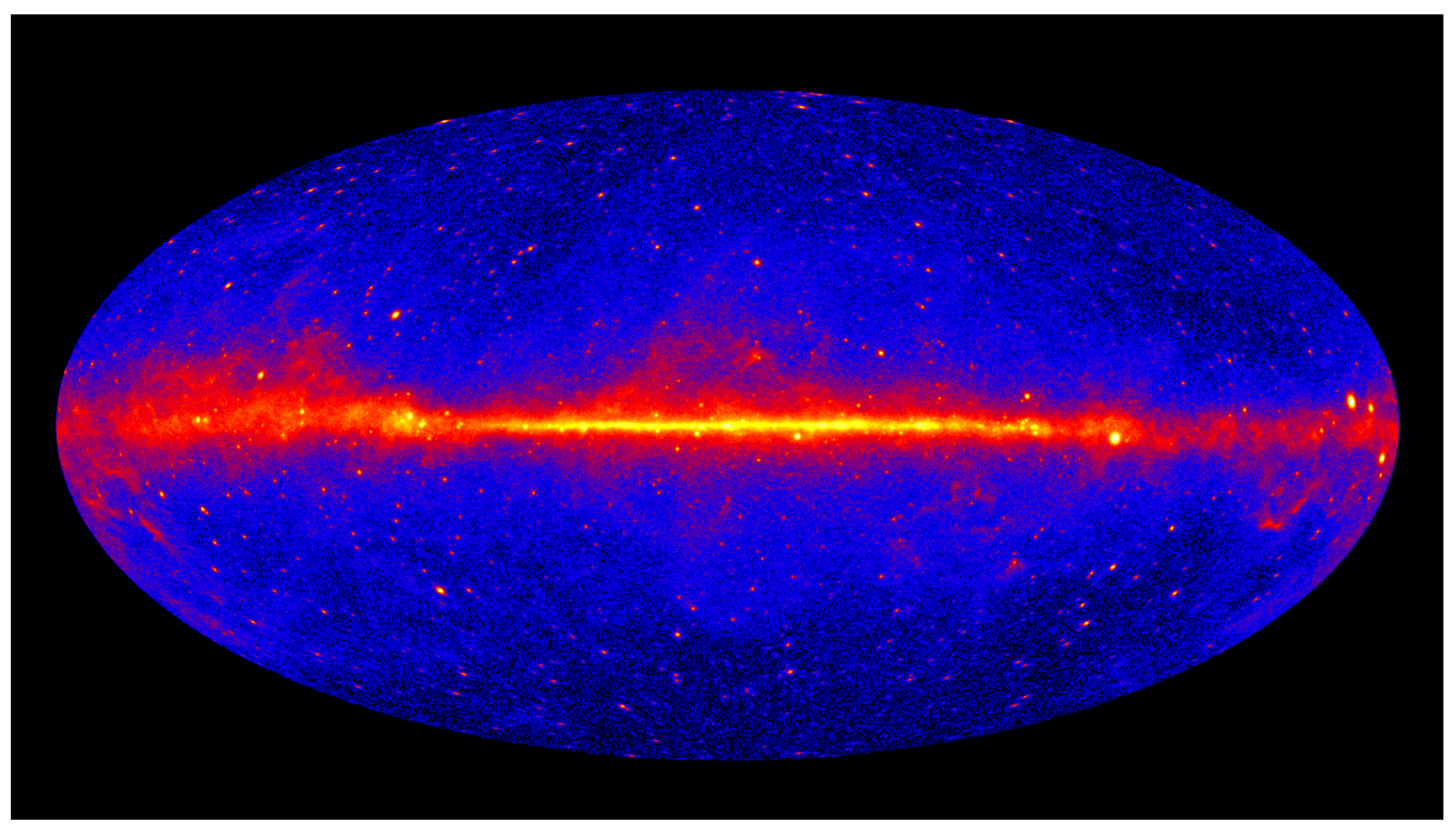

Figure 1.3: This is a Fermi-LAT all-sky view at energies $>1 \mathrm{GeV}$ created with five years of data. For better angular resolution, the map shows only gamma rays converted at the front of the instrument's tracker (See Section 1.5). Brighter colors indicate brighter gamma-ray sources. The map is shown in galactic coordinates, centralized in the GC. Source: NASA/DOE/Fermi LAT Collaboration (svs.gsfc.nasa.gov/11342).

In the most recent catalog of Fermi-LAT sources, there are 36 cataloged sources in the inner $3^{\circ}$ of the Galaxy, the most crowded region of the Fermi sky. 
Even when the emission associated with the interaction of $\mathrm{CR}$ with the interstellar gas and the cataloged point sources are taken into account, there is an excess of diffuse gammaray emission in the GC that can not be explained by the current models (Goodenough and Hooper, 2009; Ajello et al., 2016; Ackermann et al., 2017). Several interpretations for this phenomenon were sugested in the literature: self-annihilating dark matter (Calore et al. 2015; Ackermann et al. 2017, but see Abazajian et al. 2020), an unresolved population of millisecond pulsars (Abazajian and Kaplinghat, 2012; Hooper and Linden, 2016), an unresolved population of young pulsars (O'Leary et al., 2015; The Fermi-LAT Collaboration et al. 2017), or a series of periods of enhanced activity form Sgr A* caused by accretion phenomena (Petrovic et al., 2014; Cholis et al., 2015). Even interpretations related to imperfections in the GC gas distribution have been suggested (Macias et al., 2018).

In even higher energies, $\gtrsim 1 \mathrm{TeV}$, H.E.S.S. Collaboration et al. (2016) observe an extended emission centered in Sgr A* that was explained as the result of the interaction of $\mathrm{PeV}$ protons with the gas content in the central $10 \mathrm{pc}$ of the Galaxy. They also report a centrally-peaked profile for the $\mathrm{CR}$ density in the GC. They propose the presence of a petaelectronvolt accelerator ('PeVatron') of CR in the GC to explain the observations. Later, these results were confirmed by H.E.S.S. Collaboration et al. (2018), that also describe a new point source, HESS J1746-285, in the vicinity of the GC and argue that it is produced by a pulsar wind nebula candidate. MAGIC Collaboration et al. (2020) also corroborate the centrally-peaked CR profile, although their data are only marginally compatible with the PeVatron scenario, suggesting CRs with slightly lower energies. The three works, in their spatial modeling of the GC gamma-ray sky, require the inclusion of a point source coincident with Sgr A*'s position. They also suggest a more active phase of Sgr $\mathrm{A} *$ in the past as the origin of the high energy CRs.

\subsection{Sagittarius $A *$}

The SMBHs that lie in the center of every sufficiently massive galaxy manifest themselves in a myriad of different and complex ways. The majority is in a quiet state, not revealing itself in any dramatic way. Others are really conspicuous and detected as Active Galactic Nuclei (AGNs, Ho 2008). 
The center of our galaxy hosts the nearest SMBH with a mass of $4.02 \pm 0.20 \times 10^{6} M_{\odot}$ (Boehle et al., 2016) located at a distance of $8.2 \mathrm{kpc}$ (GRAVITY Collaboration et al. 2019). It is, therefore, an attractive laboratory to investigate SMBHs, even though it is in a tranquil state.

In this Section we present more details about this alluring object, Sgr A*. We start with a historical perspective about its discovery. Then, we present its current and past, characteristics.

\subsubsection{A historical perspective}

The history of the discovery of Sgr A* and its association with a SMBH is heartwarmingly recounted by Goss et al. (2003). They focus on the period up to 1985. The first observations of a source that would later be associated with Sgr A* were made by Balick and Brown (1974) in radio wavelengths.

The first use of the name Sgr A* to describe the GC compact radio source was by Brown (1982). In Goss et al. (2003), Bob Brown gives the following rationale for the name:

"Scratching on a yellow pad one morning I tried a lot of possible names. When I began thinking of the radio source as the 'exciting source' for the cluster of $\mathrm{H}_{I I}$ regions seen in the VLA maps, the name Sgr A* occurred to me by analogy brought to mind by my $\mathrm{PhD}$ dissertation, which is in atomic physics and where the nomenclature for excited state atoms is He*, or Fe* etc." (Goss et al., 2003)

By the end of the 1970s and beginning of 1980s, the discovery that the line of sight velocities of ionized gas increased to a few hundred $\mathrm{km} \mathrm{s}^{-1}$ in the central parsec of the Galaxy (Wollman et al., 1977) suggested the presence of a mass concentration in the GC (with $2-4 \times 10^{6} M_{\odot}$ ). This virial analysis of the gas lead to the conclusion that this mass concentration might be a massive black hole plausibly associated with the compact radio source Sgr A* (Lacy et al., 1980, 1982). During the 1980s, more detailed measurements of the dynamics of gas (ionized as well as of atomic and molecular) in the GC improved the case. But, since gas is sensitive to forces other than gravity, further progress required stellar dynamics. Through the 1980s, stellar velocity dispersion measurements in the GC confirmed and refined these conclusions. Genzel et al. 2010 presents a nice review about these works in Section IV-b. 
As the resolution of the observations and the quality of the dynamics tracers (diffuse gas, in the beginning, stellar velocity dispersion in the 1980s) increased, the space volume allowed for the central mass in the GC got smaller and smaller. The case for the presence of a SMBH associated with the radio source Sgr A*'s position became stronger.

The next big leap came from the detection of the first stellar proper motions. This, along with other stronger evidences of the presence of a SMBH in the center of the Milky Way, is described in Section 1.3.2.

\subsubsection{The Galactic Center black hole}

In this section, we review the most recent evidence for the presence of a SMBH in the center of Our Galaxy.

Using adaptive optics at near-infrared (NIR) wavelengths has allowed the identification and study of several stars orbiting Sgr A* in the inner arcsecond of Our Galaxy. These high velocity stars, referred as the the S-star cluster, have highly eccentric and inclined orbits. Their reconstructed orbital parameters can be used to infer Sgr A*'s mass and distance (Eisenhauer et al., 2005; Ghez et al., 2008; Gillessen et al., 2009; Boehle et al., 2016; GRAVITY Collaboration et al., 2019), test General Relativistic effects in the surroundings of a SMBH (Zucker et al., 2006; GRAVITY Collaboration et al., 2018b) and, also, study the mass distribution in the GC (Rubilar and Eckart, 2001; Mouawad et al., 2005).

The GRAVITY Collaboration has been monitoring stellar orbits in the GC in NIR wavelenghts for more than 20 years (e.g., Eisenhauer et al. 2005; Gillessen et al. 2009; GRAVITY Collaboration et al. 2019), mainly with the SINFONI and NACO adaptive optics instruments at the ESO Very Large Telescope (VLT), and since 2016 with the GRAVITY instrument. Complete orbits of the S-star cluster members have already been observed. In Figure 1.4 we show an image of the S-star cluster from Gillessen et al. (2009) with dozens of identified members.

Among these stars, S2 is one of the brightest. It has a very short orbital period around Sgr $\mathrm{A} *$ of just $\approx 16$ years and, hence, is a sensitive probe of the gravitational field in the GC. GRAVITY Collaboration et al. (2018b) reported results of $\approx 26$ years of S2 observations. Figure 1.5, shows several detections of the star through the years. Near pericenter (at $\approx 1400$ Schwarzschild radii from Sgr A*), the star has an orbital speed of $\approx 7650 \mathrm{~km} \mathrm{~s}^{-1}$, such that special relativity effects were detected. The S2 data are inconsistent with pure 


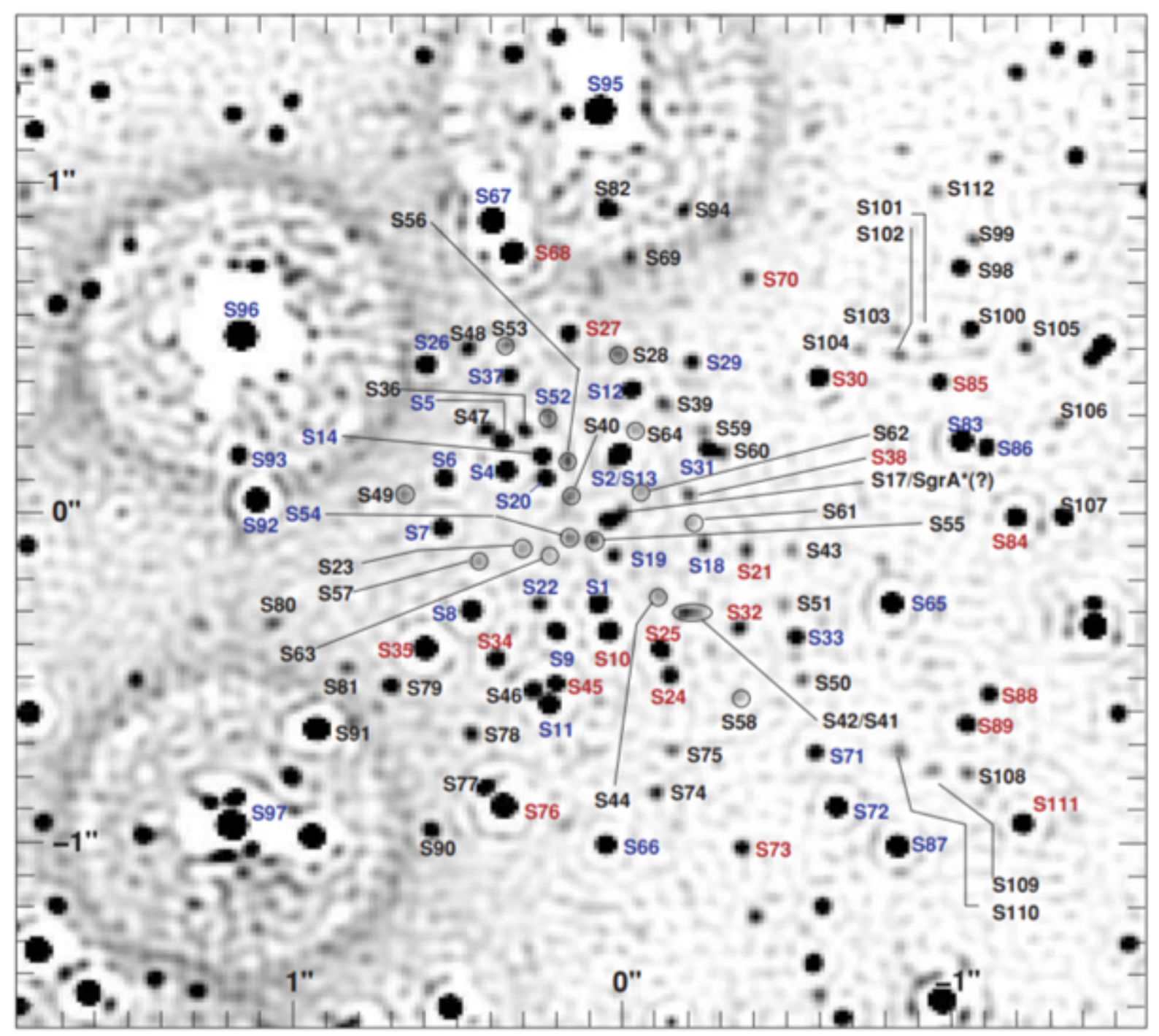

Figure 1.4: The S-star cluster. This figure is based on an image in the $\mathrm{H}$ band obtained using the NACO system mounted at the VLT array. Only stars that are unambiguously identified in several images have designated names, ranging from S1 to S112. Blue labels indicate early-type stars, red labels late-type stars. Stars with unknown spectral type are labeled in black. At the GC distance, this image is $\sim 0.14 \times 0.14$ pc. Source: Gillessen et al. (2009). 

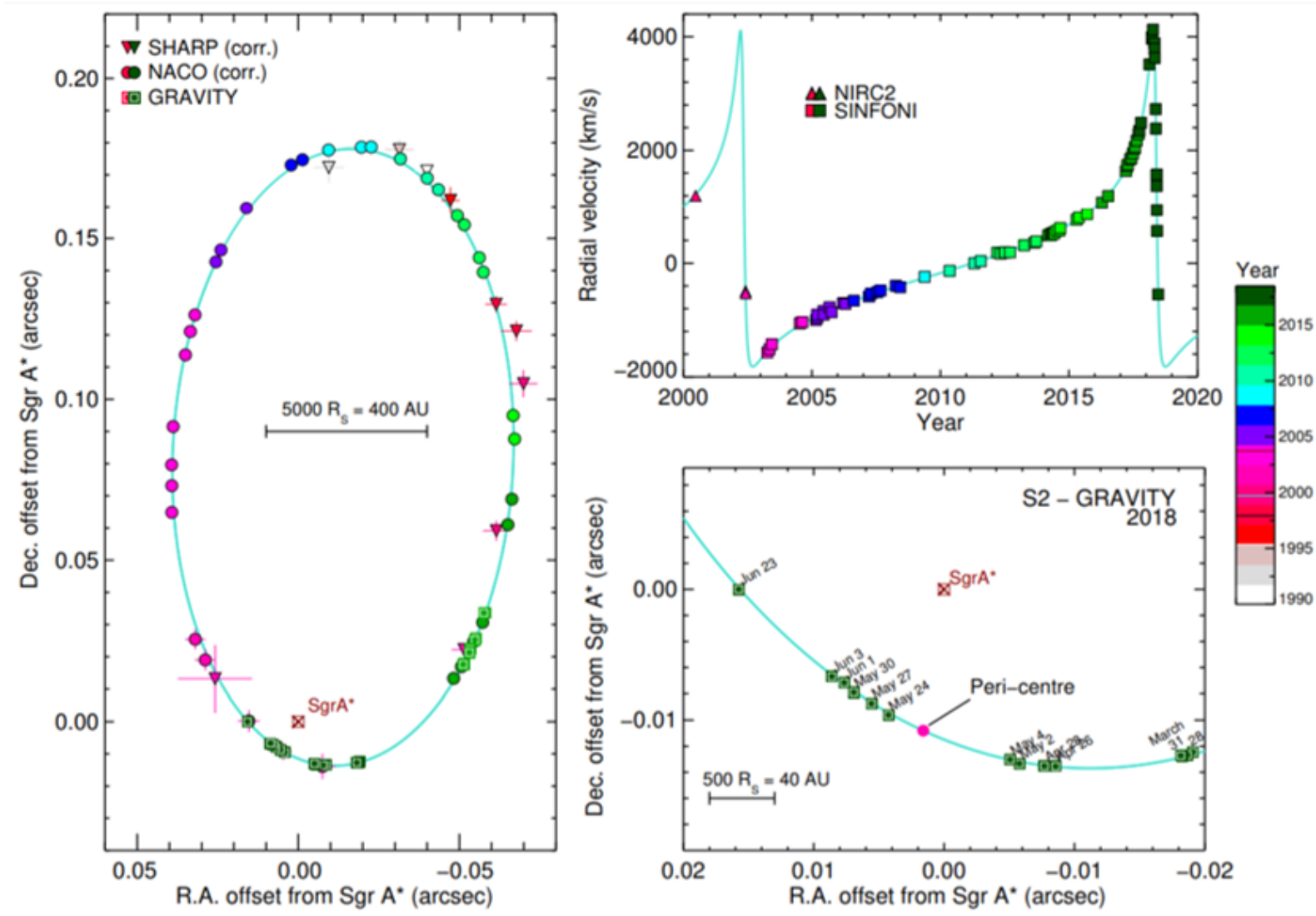

Figure 1.5: Summary of the observational results of monitoring the S2 orbit from 1992 to 2018. Left: projected orbit of the star S2 on the sky (J2000) relative to the position of the compact radio source Sgr A*. Triangles and circles denote the position measurements with different instruments, color-coded for time (color bar on the right side). The bottom right panel shows a zoom around pericenter in 2018. Top right: radial velocity of S2 as a function of time. The cyan curves show the best-fitting S2 orbit to all these data, including the effects of General Relativity. Source: GRAVITY Collaboration et al. (2018b).

Newtonian dynamics.

Recently, Peißker et al. (2020) reported indications of a population of faint fast-moving stars even closer to Sgr A* than S2. They were detected with the SINFONI and NACO instruments in the NIR. One of these stars, S4714, has an orbital period of just $~ 12$ years, an eccentricity of 0.985 and has an orbital speed of up to $\approx 24000 \mathrm{~km} \mathrm{~s}^{-1}(\approx 8 \%$ of the light speed).

Gillessen et al. (2012) detected, with VLT's instruments NACO and SINFONI observations, a dense gas cloud, with approximately three times the mass of Earth, falling inside the sphere of gravitational influence of $\mathrm{Sgr} A *$. The pericenter of this orbit was predicted to occur in the beginning of 2014 (Gillessen et al., 2013; Phifer et al., 2013). The GC community was eagerly expecting this close encounter, with hopes of observing a tidal disruption event in the heart of Our Galaxy and that G2's fragments could potentially 
increase Sgr A*'s accretion rate (Schartmann et al., 2012; Saitoh et al., 2012) leading to bursts and outflows. Several observing campaigns were organized to witness this event. Fermi, for instance, changed its observing strategy during 2014 to increase its exposure of the GC1. Unfortunately, Nature did not favor the GC enthusiasts: pericenter and postpericenter observations showed that the G2 object, after a maximum approximation of 1500 Schwarzschild radii from Sgr A*, escaped relatively unscathed. The scenario for the G2 object, thus, is more complicated then a simple gas cloud, although it was elongated by the encounter (Plewa et al., 2017).

The GRAVITY Collaboration has published several other interesting discoveries regarding the GC and Sgr A*. Among them the detection of orbital motions of "hot spots" in the accretion flow near the last stable circular orbit of Sgr A* GRAVITY Collaboration et al. 2018a), which is the observation probing the closest distances to the event horizon. These "hot spots" move at about $30 \%$ the speed of light, exhibit continuous rotation of the polarization angle with about the same period as that of their motions and are consistent with a near face-on, circular orbit of a compact polarized "hot spot" of infrared synchrotron emission at about 6 to 10 gravitational radii of Sgr A*.

Even closer observations of Sgr A* are still anxiously anticipated. Results of the EHT observational campaign to reveal the image of the shadow of Sgr A* on the accretion flow were not revealed still, due to the much shorter dynamical timescales of Sgr A* (compared do M87*) which leads to higher flux variability and to the scattering effects caused by the interstellar medium (Event Horizon Telescope Collaboration et al., 2019).

\subsubsection{Basic facts about Sgr A*}

In this Section, we report useful data about $\operatorname{Sgr} A *$. These are the values and definitions we use throughout this work, unless clearly stated:

- $\underline{\text { Mass: }} M=(4.02 \pm 0.20) \times 10^{6} M_{\odot}$ (Boehle et al., 2016).

- Distance: $d=8.2$ kpc (GRAVITY Collaboration et al., 2019).

- Schwarzschild radius: $R_{S} \approx 1.2 \times 10^{12} \mathrm{~cm}=1.2 \times 10^{7} \mathrm{~km}$.

\footnotetext{
${ }^{1}$ fermi.gsfc.nasa.gov/ssc/observations/types/exposure/
} 
- ISCO: the Innermost Stable Circular Orbit (ISCO) is defined as the smallest circular orbit in which a particle can stably orbit a black hole without falling through the event horizon. The ISCO defines the inner edge of the accretion disk around a black hole. For a non-rotating black, $R_{I S C O}=3 R_{S}$. For a black hole with Sgr A* mass, we have: $R_{I S C O} \approx 3.6 \times 10^{12} \mathrm{~cm}=3.6 \times 10^{7} \mathrm{~km}$ (it is smaller in the case of a rotating black hole).

- Bondi radius: defined as the radius where the gravitational energy owing to the black hole surpass some material's kinetic energy and, therefore, it is bound to the black hole, in a spherical accretion context. The Bondi radius, hence, gives an approximate idea of how far from the black hole an object (like stellar winds) is likely to be drawn in and accreted. For $\operatorname{Sgr} \mathrm{A} *$, the reported Bondi radius is: $R_{B} \sim 10^{5} R_{S}$ Genzel et al., 2010).

- Sphere of influence: the sphere of influence is a region around a SMBH in which the gravitational potential of the SMBH dominates that of the host galaxy. The radius of the sphere of influence is the Gravitational Influence Radius: $R_{i n f}=\frac{G M_{B H}}{\sigma_{*}^{2}}$, where G is the Gravitational Constant, $M_{B H}$ is the $\mathrm{BH}$ mass and $\sigma_{*}$ is the the stellar velocity dispersion of the galaxy bulge. For Sgr $\mathrm{A} *: R_{\text {inf }} \approx 2-3$ pc Genzel et al., 2010; Eckart et al., 2017).

- Luminosity: assuming isotropical emission, $L_{b o l} \sim 10^{36} \mathrm{erg} \mathrm{s}^{-1}$ (from radio to X-rays, Genzel et al. 2010).

- Eddington luminosity: defined as the maximum luminosity a generic object can reach before the radiation pressure generated by the emitting material surpass the gravitational force towards the center of mass. The Eddington luminosity assumes spherical accretion, hence, is used only as an order of magnitude approximation in the case of BH. For a SMBH with Sgr A* mass: $L_{E d d} \sim 5 \times 10^{44} \mathrm{erg} \mathrm{s}^{-1}$.

- Accretion rate: a characteristic of Radiatively Inefficient Accretion Flow models (used to explain Sgr A* accretion, see Section 1.3.4) is that the mass accretion rate varies with the distance to the SMBH. Genzel et al. (2010) collects several observational measurements of the accretion rate in $\operatorname{Sgr} \mathrm{A} *$. The innermost measurement $(\lesssim$ 
$\left.10^{2} R_{S}\right)$ is: $10^{-9} \lesssim \dot{M}<10^{-7} M_{\odot} / y r$. We included other values reported by Genzel et al. (2010) in Table 1.1.

- Eddington mass accretion rate: this value can obtained by solving the relation $L_{E d d}=$ $\eta_{r} \dot{M}_{E d d} c^{2}$, where $\eta_{r}$ is the radiative efficiency of the acretion process. If we consider $\eta_{r} \sim 0.1$, we get: $\dot{M}_{E d d} \approx 9 \times 10^{-2} M_{\odot}$.

Table 1.1 - Overview of the accretion rate as a function of distance from the Galactic Center black hole.

\begin{tabular}{ccc}
\hline \hline Region & Radius & $\begin{array}{c}\text { Mass accretion rate } \\
\left(M_{\odot} / y r\right)\end{array}$ \\
\hline Giant molecular clouds & Tens to few hundreds of parsec & $10^{-2}$ \\
Circum nuclear disk & $1.7-7 \mathrm{pc}$ & $10^{-3}-10^{-4}$ \\
Central cavity, minispiral, and stellar cluster & $<1.7 \mathrm{pc}$ & $10^{-3}-10^{-4}$ \\
Stellar winds at Bondi radius & $0.05 \mathrm{pc}=10^{5} R_{S}$ & $\mathrm{~A}$ few $10^{-6}$ \\
Outer accretion zone & $10^{2}-10^{3} R_{S}$ & $<10^{-6}$ \\
Inner accretion zone & $\mathrm{A}$ few to $10^{2} R_{S}$ & $\mathrm{~A}$ few $10^{-9}-10^{-7}$ \\
\hline
\end{tabular}

Source: Genzel et al. (2010)

\subsubsection{Multiwavelength emission}

The GC is the closest example of a galactic nucleus and hence has been the subject of observational scrutiny with hopes of understanding the environment close to the SMBH. The electromagnetic radiation from Sgr A* has been detected in several wavelengths (e.g., Genzel et al. 2010; Morris et al. 2012; Eckart et al. 2018), with the exceptions being the visible band and gamma-rays.

The obstacle to observe the GC in visible spectrum is the presence of considerable amount of dust and gas in the Galactic disk, which is in the line of sight. Seminal spectrophotometric studies show that the GC is shrouded by $\sim 30$ magnitudes in visible wavelengths as light travels through the interstellar medium (Becklin and Neugebauer, 1968 Becklin et al., 1978; Rieke and Lebofsky, 1985; Rieke et al., 1989).

The hindrance in observing gamma-rays is from a different nature. Gamma-ray emission has been detected coincident with the position of Sgr A*. But, given the large pointspread function (PSF) of astronomical gamma-rays detectors (as an example, in Section 1.5.3, especially Figure 1.14, we characterize Fermi-LAT's PSF), associating this emission to a single source is very challenging. The diffuse gamma-ray emission from the GC region was presented in Section 1.2.1 and the emission spatially coincident with Sgr A* is 
comprehensively discussed in this thesis.

In this Section we discuss the observations of Sgr A* across the electromagnetic spectrum. First, we center on its potential gamma-ray emission (Section 1.3.3.1). Then, we focus on its different states (Section 1.3.3.2), its variability (Section 1.3.3.3), and finish showing evidences of past periods of activity (Section 1.3.3.4).

\subsubsection{Sagittarius $A *$ in gamma-rays}

Gamma-ray emission (in the $\mathrm{MeV}$ to $\mathrm{TeV}$ energy range) coincident with the position of Sgr A* has been reported through the years. But no unique Sgr A* counterpart has been reported in the gamma-ray domain, given the low spatial resolution of gamma-ray telescopes. In the next paragraphs, we mention several astrophysical gamma-ray detectors. We refer the reader to Sections 1.5 and 1.4 for more technical details about Fermi and other gamma-ray instruments (EGRET, VERITAS, H.E.S.S. and MAGIC), respectively.

In this Section, we give a brief overview about these observations. Here, we are talking about emission potentially associated with the SMBH. In Section 1.2.1 we describe gammaray emission from the GC as a region, including diffuse emission and other likely point sources.

The bright EGRET source 3EG J1746-2851 is listed in the third EGRET catalog (Hartman et al. 1999) with an $95 \%$ confidence contour radius of $0^{\circ} .13$, is consistent with $\mathrm{Sgr}$ A*'s position and was considered as the gamma-ray counterpart to the GC region (MayerHasselwander et al., 1998). However, an independent analysis of the EGRET data by Hooper and Dingus (2005) indicates a point source whose position is different from Sgr $\mathrm{A} *$ at a confidence level beyond 99.9\%. Later, 3EG J1746-2851 was associated with Sgr A East, with the gamma-ray emission arising from the decay of neutral pions produced by high-energy protons, accelerated in the supernova remnant, interacting with the ambient matter (Fatuzzo and Melia, 2003).

Also, sub-TeV gamma-ray emission from the direction of the GC was detected with CANGAROO-II Imaging Atmospheric Cerenkov Telescop $2^{2}$ (Tsuchiya et al., 2004). The centroid was consistent with Sgr A* position and compatible with a point source. Sgr A*

\footnotetext{
${ }^{2}$ The CANGAROO (Collaboration between Australia and Nippon for a Gamma-Ray Observatory in the Outback) Project operated a series of Imaging Air Cherenkov Technique gamma-ray telescopes from a previous generation (Mori, 2003).
} 
was considered as a potential association for this emission.

Another gamma-ray detection from the GC suggesting an association with Sgr A* was performed with the Whipple 10-meter gamma-ray telescope, a predecessor of VERITAS. Kosack et al. (2004) report a possible detection of TeV gamma rays from the GC. The 95\% confidence region has an angular extent of about $0 .^{\circ} 15$ and includes the position of Sgr A*. The detection is consistent with a point source and shows no evidence of variability.

Aharonian, F. et al. (2004) reported the detection of a point source of very high energy gamma-rays coincident within 1' with Sgr A*, obtained with H.E.S.S.. More recently, TeV emission observed by H.E.S.S. indicates the presence of $\mathrm{PeV}$ protons within the central 10 pc of the Galaxy (H.E.S.S. Collaboration et al., 2016). They propose that a more active phase of Sgr A* in the past could have accelerated this population of high-energy protons. H.E.S.S. Collaboration et al. (2018) reported the detection of an extended central component around Sgr A* that most likely originates from an enhancement of CRs in the inner tens of pc around the GC, thereby confirming the results presented in H.E.S.S. Collaboration et al. (2016).

Albert et al. (2006) confirmed, with MAGIC observations, the gamma-ray source at the GC. Ahnen, M. L. et al. (2017) present results of a monitoring campaign of the GC between 2012-2015. They identified a point-like gamma-ray excess whose location is spatially consistent with Sgr A* as well as SgrA East. Updated MAGIC observations of the GC also corroborates the previous observations (MAGIC Collaboration et al., 2020).

Following the H.E.S.S. Collaboration et al. $2016 \mathrm{TeV}$ detections of the GC, several candidates have been proposed for this gamma-ray flux: Sgr A* itself, either from its immediate vicinity (Aharonian and Neronov, 2005a) or from a "plerion" produced by the SMBH winds (Atoyan and Dermer, 2004; Kusunose and Takahara, 2012); the interaction between the dense molecular clouds in the GC with cosmic rays accelerated by Sgr A* and/or by some other nearby source Aharonian and Neronov, 2005b; Ballantyne et al. 2011; Chernyakova et al., 2011; Linden et al., 2012; Fatuzzo and Melia, 2012; Guo et al. 2013); the pulsar wind nebula (PWN) G359.95-0.04 (Wang et al., 2006; Hinton and Aharonian, 2007); the supernova remnant Sagittarius A East (Crocker et al., 2005) (but see

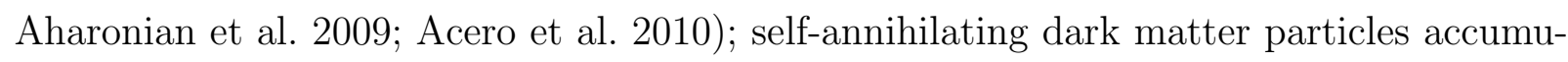
lating at the GC (Hooper and Goodenough, 2011; Hooper and Linden, 2011) and an as-yet undetected pulsar (or population of pulsars) (Hooper and Linden, 2011). 
Prominent gamma-ray emission from $\mathrm{MeV}$ to $\mathrm{TeV}$ energies coincident with Sgr A*'s position is observed by Fermi-LAT. Since the beginning of Fermi's operations, a point source has been observed coinciding with the position of Sgr A*. This source was studied by Chernyakova et al. (2011) with 25 months of Fermi observations. They found no temporal variability at $\mathrm{GeV}$ energies and proposed a model in which the gamma-ray emission in the inner 10 pc of the Galaxy arises from relativistic proton interactions. Later, Malyshev et al. (2015) analyzed the same source using 74 months of data and the Second Catalogue of Fermi LAT Sources (Nolan et al., 2012). They also found no variability in the flux, and considered the observed spectrum as consistent with Inverse Compton (IC) scattering of high-energy electrons.

Ahnen, M. L. et al. (2017) collected several models for the MeV to TeV emission from Sgr A*. The list includes leptonic (Kusunose and Takahara, 2012), hadronic (Fatuzzo and Melia, 2012; Linden et al., 2012; Chernyakova et al., 2011) and hybrid (Guo et al., 2013) models. We call the models in this list "Fermi-era", since they were all constructed taking into account Fermi-LAT's data.

Despite all these observations, the nature of the gamma-ray emission from the direction of Sgr A* is still a matter of debate. Its spectrum and variability is so far unconstrained. And a solid association between this emission and Sgr A* is yet to be established.

\subsubsection{The two states of Sagittarius A*}

Most of the time, $\mathrm{Sgr} \mathrm{A} *$ is in a steady state, emitting $\sim 10^{36} \mathrm{erg} \mathrm{s}^{-1}$ (Genzel et al., 2010), between radio wavelengths and X-rays. This emission is dominated by the radioto-sub-mm emission. On top of the steady emission, there is also a variable component in X-rays, near-infrared and also in longer wavelengths.

Given the surplus of gas reservoirs in the GC region, Sgr A* is remarkably under luminous. Its luminosity is more than 8 orders of magnitude smaller than $L_{E d d}$ and $\sim 4$ orders of magnitude lower than the expected luminosity ${ }^{3}$ estimated based on the accretion rate at its Bondi radius (a few $\times 10^{-6} M_{\odot} y r^{-1}$, Baganoff et al. 2003; Xu et al. 2006). The luminosity of Sgr A* is dominated by accretion from stellar winds in its vicinity. In addition, larger accretion rates probably occur from time to time due to stochastic density

\footnotetext{
${ }^{3}$ Calculated as $L_{B}=0.1 \times \dot{M}_{B} c^{2}$, where $\dot{M}_{B}$ is the Bondi accretion rate and assuming a $10 \%$ efficiency rate
} 


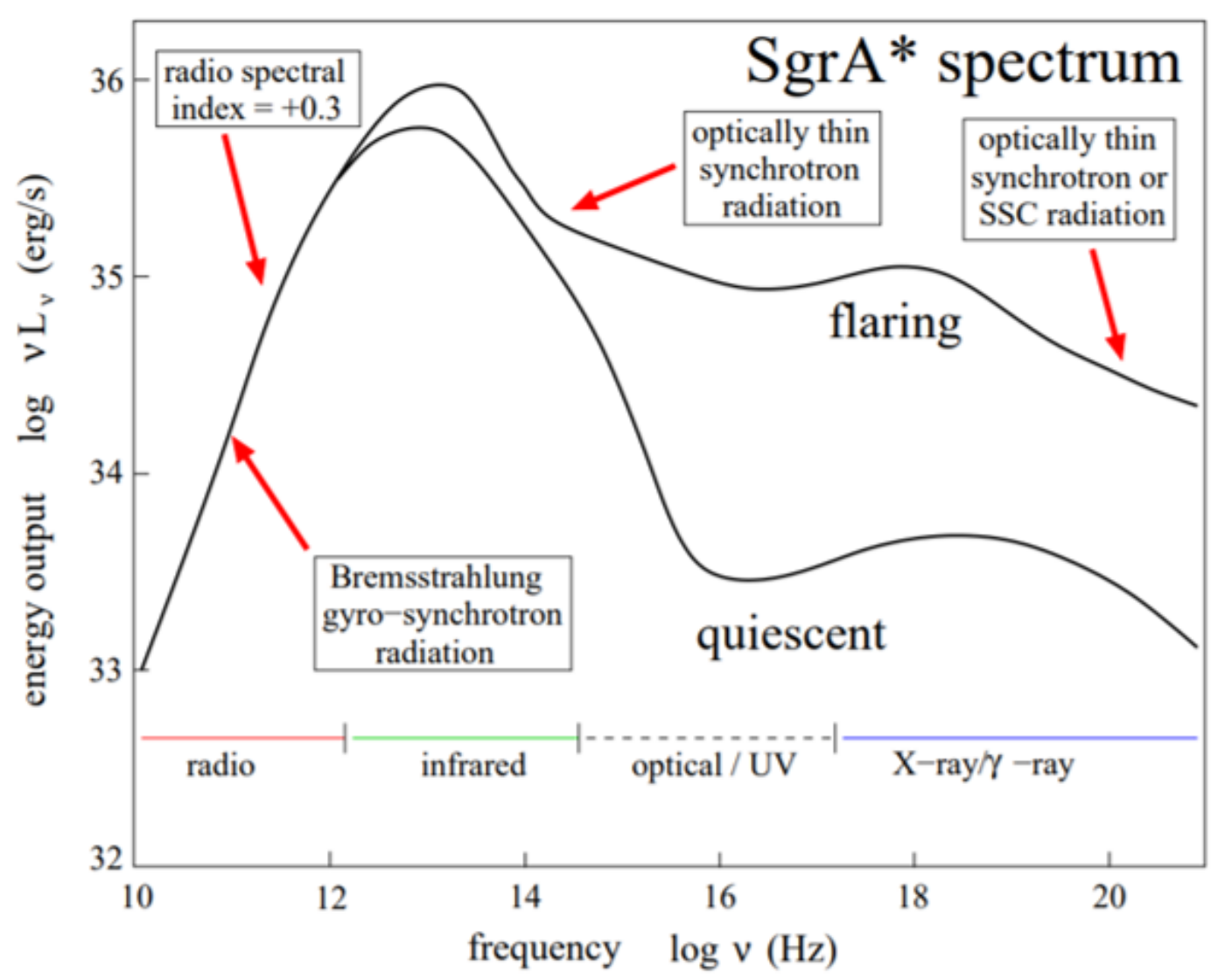

Figure 1.6: Sgr A* spectrum in quiescent and flaring states. Some of the prominent radiation mechanisms are highlighted. This plot was created based on models from Yuan et al. (2003); Narayan et al. (1998); Narayan and McClintock (2008) and using data from several works (radio from Falcke et al. 1998: Zhao et al. 2003. IR from Eckart et al. 2018 and references in there; and X-ray from Baganoff et al. 2001, 2003

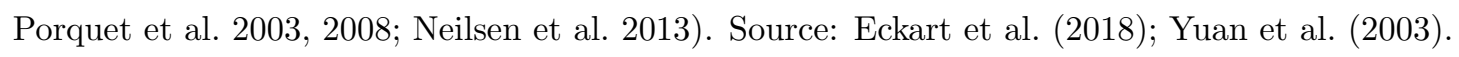

fluctuations in the accretion disk, or when large gas clouds with low angular momentum fall into the center.

Sgr A* has been detected in most of the electromagnetic spectrum (e.g., Dibi et al. 2014). In Figure 1.6 we show a sketch, created by Eckart et al. (2018) based on Yuan et al. (2003) work, of Sgr A*'s quiescent and flaring broad band spectrum. Some of the prominent radiation mechanisms are highlighted (see Section 1.3.4 for more details).

The compact radio source Sgr A*'s radio emission, discovered in the 1970s, is clearly detectable at all times and shows only moderate flux and spectral variations. The variable NIR source associated with the accretion flow around Sgr A* was unambiguously identified in the first years of this millennium (Genzel et al., 2003; Ghez et al., 2004). Twenty two years of NIR data collected from Sgr A* shows consistent brightness and variability during the whole period (Chen et al., 2019). This emission is consistent with synchrotron radiation 
from a non-thermal electron population (Witzel et al., 2018). A steady and faint X-ray counterpart do Sgr A* was first reported by Baganoff et al. (2003). The quiescent emission level is likely to arise relatively far from the black hole, at the Bondi accretion radius. There, the accretion flow undergoes shocks and thermalizes, producing bremsstrahlung X-rays Quataert et al., 2002; Xu et al., 2006).

\subsubsection{Variability and flares}

Sgr A* is highly variable in the infrared Genzel et al., 2003; Ghez et al., 2004; Hornstein et al., 2007; Dodds-Eden et al., 2009; Witzel et al., 2012; Hora et al., 2014; Witzel et al., 2018; von Fellenberg et al., 2018; Fazio et al., 2018; Boyce et al., 2019) and X-rays (Baganoff et al., 2001; Nowak et al., 2012; Neilsen et al., 2013; Barrière et al., 2014; Neilsen et al., 2015; Ponti et al., 2015; Fazio et al., 2018; Boyce et al., 2019), which suggests a compact source. One can put an upper limit (UL) to the size of the region from which a flare originated by calculating the light crossing time since any disturbance that originated the flare must have spread through the source with speeds lower than light's. Variability has also been reported in longer wavelengths (Zhao et al., 2003; Miyazaki et al., 2004; Mauerhan et al., 2005; Macquart et al., 2006; Yusef-Zadeh et al., 2006; Marrone et al., 2008; Yusef-Zadeh et al., 2009; Plambeck et al., 2014; Brinkerink et al., 2015; Stone et al., 2016). The gammaray flux, though, does not seem to be variable (Chernyakova et al. 2011; Malyshev et al. 2015; Ahnen, M. L. et al. 2017 and Sections 3.3 and 4.2 of this thesis) and there is still no definitive association between this emission and Sgr A* (Section 1.3.3.1).

Typically, about one X-ray flare is generated per day with duration of a few tens of minutes Neilsen et al. (2013). The brightest observed X-rays flares are $\sim 100$ times above the quiescent level (e.g., Nowak et al. 2012). The NIR flares are even more frequent. X-ray flares usually follow, after a few tens of minutes, the NIR ones, but there are multiple NIR flares without X-rays counterparts (e.g., Eckart et al. 2006; Yusef-Zadeh et al. 2012; Ponti et al. 2017; but see Fazio et al. 2018). Flares are also observed in millimeter and sub-mm wavelengths (e.g., Yusef-Zadeh et al. 2006; Stone et al. 2016). They last from hours to days with amplitudes of $\sim 25 \%$ of the quiescent level (Yusef-Zadeh et al., 2008; Fazio et al., 2018).

Flaring emission is an indicative that some accretion, heating, or magnetic reconnection mechanism has given rise to enhanced emission from the vicinity of the SMBH. They 

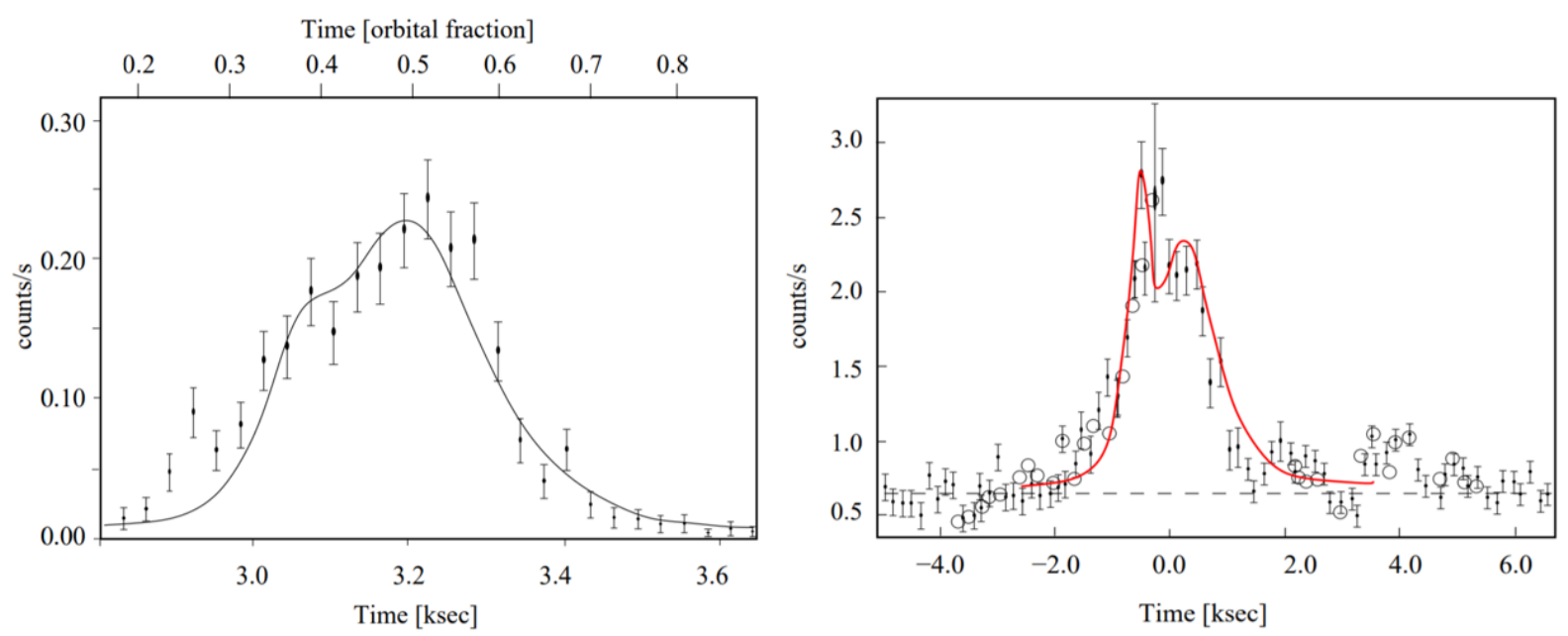

Figure 1.7: Fit of the hotspot model of Karssen et al. (2017) to two bright X-ray flares. Left: flare published by Nowak et al. (2012). Right: flare reported by Ponti et al. (2017). In these plots, the "shoulder" is due to gravitational lensing when the hotsopt is behind the black hole. The peak is due to boosting when the hotspot approaches the observer after passing around the black hole.

OBS: the horizontal axis in the left panel is incorrect. The scale should be of $10^{4} \mathrm{~s}$, as in Figure 1 of Nowak et al. (2012). Source: Eckart et al. (2018).

are, consequently, subject to relativistic effects. Based on General Relativistic Magnetohydrodynamics (GRMHD) simulations, short term variability is often explained by hotspot emission from the accretion disk which can be produced, for example, by magnetic reconnection (Meyer, L. et al., 2006a.b; Eckart et al., 2006; Broderick and Loeb, 2005, 2006; Ball et al. 2016). Close to the SMBH, the interplay of relativistic effects may influence the appearance of flare emission (Doppler boosting, gravitational redshift, light focusing, and light-travel time delays, e.g. Broderick and Loeb 2005, 2006; Eckart et al. 2006). In some bright X-ray flares these characteristic shapes may be visible (Nowak et al., 2012, Karssen et al., 2017; Ponti et al., 2017).

Figure 1.7, obtained from Eckart et al. (2018), shows the data of two bright X-ray flares fitted with a hotspot flare model from Karssen et al. (2017). In these plots, the "shoulder" is due to gravitational lensing when the hotsopt is behind the black hole. The peak is due to boosting when the hotspot approaches the observer after passing around the black hole. Since bright X-ray flares are often seen (almost) synchronous with bright NIR-flares, this model is probably also applicable for NIR-flares. Also, the Karssen et al. (2017) model indicates that, at least for the bright x-ray flares, the flare duration is coupled to the dynamical time scale of the hotspot (see the top horizontal axis on the left panel of Figure 1.7). 
The NIR variability can be modeled as a purely random process (Meyer et al., 2008; Do et al., 2009; Dodds-Eden et al., 2011; Witzel et al., 2012). Polarimetric observations indicate that the NIR emissions are consistent with a hotspot model (Eckart et al., 2006; Meyer, L. et al., 2006a, b, 2007; Trippe et al., 2007; Zamaninasab, M. et al., 2010).

Numerous X-ray flares have been observed since the discovery of the GC X-ray source (e.g., Baganoff et al. 2001; Porquet et al. 2003, 2008; Nowak et al. 2012; Zhang et al. 2017; Ponti et al. 2017; Boyce et al. 2019; Haggard et al. 2019). Their variability time scales, including temporal structure during the flares (see Figure 1.7), indicate that they are generated much closer to the SMBH than the quiescent emission, probably within a few tens of Schwarzschild radii. They typically last for $\sim 30 \mathrm{~min}$ and their peak fluxes range between a few and tens of times the quiescent flux. The rate of detectable flares is of about once per day. But, this may be limited by sensitivity: weaker flares possibly go undetected and contribute to the mean detected quiescent flux.

Most studies have focused on variability in the X-ray and IR, where variations are often simultaneous, and because long time series at sub-mm and radio wavelengths are limited. Nevertheless, Sgr A*'s radio variability has been studied for several decades. They indicate that the flares time scales vary from hours to days (Bower et al., 2002, Zhao et al., 2003; Herrnstein et al., 2004; Miyazaki et al., 2004). Mauerhan et al. (2005) performed observations at a wavelength of $3 \mathrm{~mm}$ and characterized the variability as a red noise process with amplitudes up to 40\%. Marrone et al. (2006) reported strong variability of both total intensity and polarization fraction on a time scale of hours at $880 \mu \mathrm{m}$.

Comparing Sgr A*'s light curves in different wavelenghts can generate clues to the nature of the emission processes. Yusef-Zadeh et al. (2006) observed two frequencies simultaneously: 22 and $43 \mathrm{GHz}$. Their light curve shows show a delay of 20 to $40 \mathrm{~min}$ of the $22 \mathrm{GHz}$ light curve, relative to $43 \mathrm{GHz}$ one. They explained this with an expanding plasma model in which the plasma cools and becomes optically thin as it expands, and thus reaches an emission maximum, at progressively lower frequencies with time. YusefZadeh et al. (2008) observed Sgr A* in the same frequencies and also in sub-mm and X-ray wavelengths. The X-ray flare precede the sub-mm flare by about $90 \mathrm{~min}$, as the expanding plasma model would qualitatively predict, and again that the $22 \mathrm{GHz}$ emission was lagging the $43 \mathrm{GHz}$ emission.

There are a plethora of observations claiming delays of a few hours between NIR flares 
and subsequent, associated, mm/sub-mm peaks: Eckart et al. (2006, 2008, 2009); YusefZadeh et al. (2006, 2008, 2009); Meyer et al. (2008); Morris et al. (2012) (but see Fazio et al. 2018). Collectively, the delays are $\sim 150 \mathrm{~min}$, consistent with an expanding relativistic plasma blob model if the expansion occurs at $\sim 0.1 \mathrm{c}$ Marrone et al., 2008; Yusef-Zadeh et al., 2009).

The expanding plasma model would predict that X-ray and near-IR emission peaks should occur simultaneously, since they are both optically thin throughout the expansion. There are observations that seem to favor this relation. It appears that every X-ray flare corresponds reasonably well in time with a well-defined and relatively bright maximum in the infrared light curve (Eckart et al., 2004, 2012; Ghez et al., 2004; Marrone et al., 2008; Dodds-Eden et al., 2009; Trap et al., 2011). The reverse is not true: only a fraction of the IR maxima correspond with an X-ray flare. Yusef-Zadeh et al. (2012) reanalyzed a body of existing data and reported of a significant time lag between the infrared maxima and the X-ray flares of a few to tens of minutes. These results are more compatible with a model in which the NIR events occur in the inner regions of the accretion flow while the X-ray flares are the result of IC scattering of the NIR photons by thermal electrons in the accretion flow, possibly at a greater radius, which causes the delay (Yusef-Zadeh et al. 2009; Wardle, 2011). Combining the mechanisms responsible to Sgr A* flares in different wavelengths is challenging and maybe distinct process are taking place.

Using the Keck telescope, Do et al. (2019) observed a flare from Sgr A* with unparalleled NIR flux, exceeding the historical peak value by a factor of two. Subsequently, the light curve exhibited a drop in the measured flux of a factor of 75 in a period of two hours. The authors suggested that the flare was a consequence of an increase in the SMBH mass accretion rate, possibly resulting from the deposition of additional gas coming from a "windy" star (e.g., S2) or the passage of the G2 object in 2014. It was argued by Ressler et al. (2018), however, that S2 should have a negligible effect on the Radiatively Inefficient Accretion Flow (see Section 1.3.4) structure. Moreover, there are no other stars from the Sstar cluster that are both close to Sgr A* and more massive than S2. Thus, the framework of a "windy" star is likely flawed. In Gutiérrez et al. (2020), in which the PhD candidate is a coauthor, a completely different scenario is proposed-independent of an increase in the mass accretion rate. Analogously to a nonthermal bomb, we suggest that the flare was the result of particle acceleration to nonthermal energies, leading to an explosive event in 
the innermost parts of the accretion flow. The NIR light curve is quantitatively explained by the proposed model, and testable predictions are also suggested at other wavelengths.

For the last few hundred of years, Sgr A* is in a state of very low activity, a consequence of its current extremely low accretion rate. But there are hints showing that Sgr A* was much more active in the past, as we discuss in the following Section.

\subsubsection{Hints of past activity of Sagittarius A* emission}

Although Sgr A* exhibits daily flares, it is in a very quiet phase (Section 1.3.3.2). But there is tantalizing evidence for an enhanced level of activity in the recent past of Sgr A*. Using 1.66 year of Fermi-LAT data Su et al. (2010) detected two large gamma-ray bubbles, extending $50^{\circ}$ above and below the Galactic plane, with a width of about $40^{\circ}$ in longitude (Figure 1.8). The Fermi Bubbles should have formed 1-3 Myr ago during a large episode of energy injection in the GC that lasted 0.1-0.5 Myr (Yang et al., 2018). The origin of the bubbles is still debated and could be due to a previous nuclear starburst or some past accretion event of the SMBH. Herold and Malyshev (2019) studied the base of the Fermi Bubbles with 9 years of Fermi-LAT observations. They reported that it is shifted to the west (negative longitudes) from the GC. If the emission at the base of the Bubbles is indeed connected to the high-latitude Bubbles, then this shift disfavors models in which they are created by $\operatorname{Sgr} \mathrm{A} *$.

Further evidence for higher levels of activity in Sgr A* comes from X-ray observations of circumnuclear clouds (Ponti et al. 2010). Concretely, X-ray observations since the 1990s show rapid variations in the $6.4 \mathrm{keV}$ of $\mathrm{Fe} \mathrm{K} \alpha$ line propagating through molecular clouds in the inner Galactic regions. These variations are likely the result of a highly variable active phase of Sgr A* within the past few hundred years, which are echoing through the clouds. Models indicate at least two luminous outbursts ( 100 and 400 years ago) on few-year

timescales during which the luminosity of Sgr A* went up to at least $10^{39} \mathrm{erg} \mathrm{s}^{-1}$ (Ponti et al. 2010; Clavel et al., 2013). In summary, it seems that Sgr A* was $10^{3}$ times more active within the past few centuries compared to current levels. Considering the estimated energy of these events, Sgr A* is a very promising candidate for their origin. 

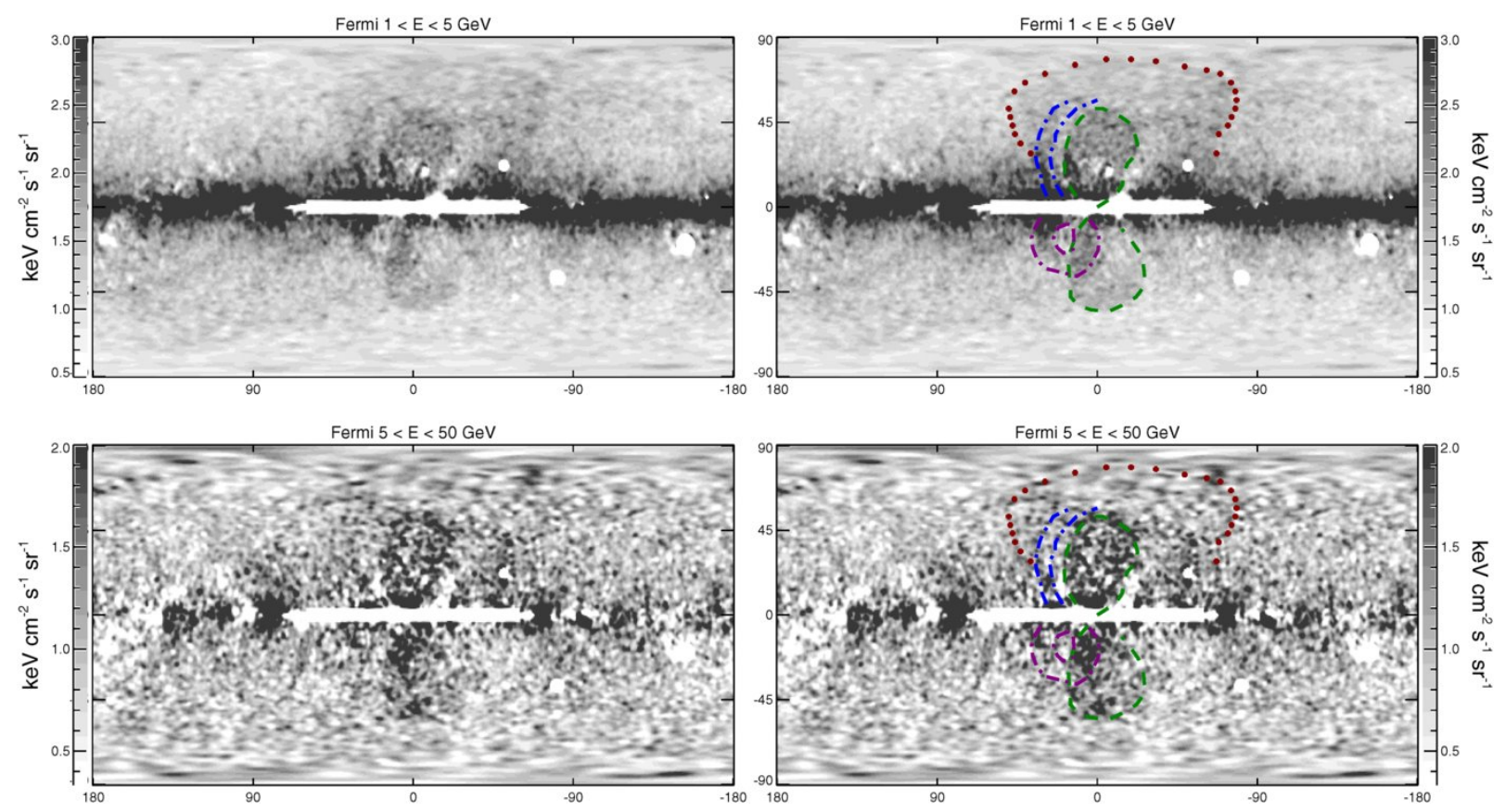

Figure 1.8: Full sky residual maps after subtracting dust and disk templates from the Fermi-LATmaps in two energy bins. Point sources are subtracted, and large sources, including the inner disk, have been masked. Two large bubbles are seen in both cases. Right panels: Apparent Fermi Bubbles features marked in color lines, overplotted on the maps displayed in the left panels. Source: Su et al. $(2010)$.

\subsubsection{Models for Sagittarius A* emission}

As mentioned in Section 1.3.3.2, if gas was accreting into Sgr A* via optically thick, geometrically thin accretion disk - a model successfully used to to explain accreting sources, like blazars (Koratkar and Blaes, 1999) - , its expected luminosity (based on the accreting rate at the Bondi radius) would be $\sim 4$ orders of magnitude higher than the observed value. This indicates that an optically thick, geometrically thin accretion disk is not compatible with Sgr A* behavior. Another argument is the lack of any any disk-like blackbody emission component in the spectrum of the SMBH (Narayan, 2002). The observational data of the quiescent state of Sgr $\mathrm{A} *$ (from radio to X-rays) favor models in which very little of the gravitational potential energy of the inflowing gas is radiated away: the Radiatively Inefficient Accretion Flow (RIAF). These kind of models have been the most successful in fitting Sgr A*'s spectrum.

The RIAF model describes the dynamics of rotating accretion flows in which much of the gravitational potential energy of the accreting material is not radiated away (e.g., Narayan et al. 1995; Yuan et al. 2003, 2004; Yuan and Narayan 2014) but is, instead, stored as thermal energy. For this reason, the temperatures in the accreting gas are very high. 
The electron temperature depends on the processes responsible for heating them (shocks, turbulence, magnectic reconnection) and, thus, are difficult to be precisely estimated. Since collisions are unimportant in the GC environment (at such high temperatures and low gas densities, the Coulomb collision time is much longer than the gas accreting time) the electron distribution function is not expected to be thermal.

Numerical simulation of RIAFs (e.g., Stone et al. 1999; Igumenshchev and Abramowicz 1999, 2000; Igumenshchev et al. 2000; Hawley and Balbus 2002; Igumenshchev et al. 2003; Sądowski et al. 2013; Almeida and Nemmen 2020) have shown that $\dot{M}<<\dot{M}_{B}$ (where $\dot{M}$ is the actual accretion rate in the $\mathrm{SBMH}$ ), which means that a very small fraction of the mass available at large radii is effectively accreted. The implication is that in the RIAF models, rather than just a low efficiency, a low accretion rate is also contributing to the SMBH faintness.

To allow for accretion in a RIAF context, there must be non-radiative energy loss of some kind. One way the binding energy of the accreting matter can be carried away is in the form of an outflow ('winds') (Quataert, 2003).

See Table 1.1 for an overview of the accretion rate towards several different radii from Sgr A*.

The relative faintness of Sgr A* has been explained through RIAF models (e.g., Narayan et al. 1995; Yuan et al. 2003, 2004). The broadband spectrum is dominated by the radio-tosub-mm emission which is understood as synchrotron radiation from a thermal population of electrons, with temperatures between $\sim 5-20 \mathrm{MeV}$, as well as a small fraction (a few percent) of nonthermal electrons (Yuan et al., 2003). The observed polarization in the submm domain argues for a low accretion rate in the inner few parsecs of $\dot{M}<10^{-7} M_{\odot} / y r$ (Mezger and Wink, 1986; Morris and Serabyn, 1996, Marrone et al., 2006).

The Sgr A*'s X-ray emission has been separated into two components Baganoff et al., 2001, 2003): a nearly constant component and a flaring one. The quiescent component is extended, with size of $\approx 10^{5} R_{S}$ (the Bondi accretion radius), and is explained by thermal bremsstrahlung originating from shocks in the transition region between the ambient medium and the accretion flow (composed by material from stellar winds) Quataert et al. 2002 ; Xu et al., 2006).

The flaring component is not extended and, given the duration and shape of the flares, is compatible with emission arising from the close vicinity of Sgr A*. There is evidence 
that the X-ray flare emission is due to synchrotron processes (Dodds-Eden et al., 2009; Barrière et al., 2014, Ponti et al., 2017) although they have also been interpreted as IC upscattered photons by the mildly relativistic, nonthermal electrons (Yusef-Zadeh et al. 2009; Ball et al., 2016). The NIR component is associated with the accretion flow around Sgr A* Genzel et al., 2003; Ghez et al., 2004; Chen et al., 2019) and its variable component is compatible, as is the case for the X-ray variability, with hotspot model.

\subsection{Other gamma-ray telescopes}

Since Earth's atmosphere is opaque to gamma-ray, astronomers have developed two kinds of instruments to make astrophysical gamma-rays observations attainable: spacebased (from which FermiLAT is an example) and Cherenkov telescopes. Both approaches are complementary. Astrophysical gamma-rays energy spans from $\sim 100 \mathrm{keV}$ to $\sim 100 \mathrm{TeV}$, which comprehends 9 decades in energy, with flux rapidly decreasing towards high energies. It is necessary, thus, to have instruments with larger effective areas to detect significant flux in higher energies. Unfortunately, the cost to send instruments to space limits the size (and hence, the effective area) of gamma-ray space telescopes making them appropriate to operate only up to the $\sim 100 \mathrm{GeV}$ energy range, as is the case of Fermi-LAT. The larger effective areas necessary to operate above these energies are only attainable by groundbased instruments. They take advantage of the electromagnetic air showers formed when gamma-rays enter the atmosphere. They are the result of a cascade of secondary particles formed when a gamma-ray converts into pairs of $\mathrm{e}^{-}$and $\mathrm{e}^{+}$at high altitude. These particles radiate secondary gamma-rays mostly through bremsstrahlung, which further convert into $\mathrm{e}^{-} \mathrm{e}^{+}$pairs with lower energies. These particles can be detected when they reach the ground or through the Cherenkov radiation they emit. Showers formed by gamma-rays with energies $\gtrsim 30 \mathrm{GeV}$ start to become detectable.

In Figure 1.9 we reproduce the Figure 3 from de Angelis and Mallamaci (2018) in which they show the point source continuum differential sensitivity of different gamma-ray (and X-ray) instruments for a source at high Galactic latitude. It is clear how the space-based and ground-based approaches are complementary. The ground-based telescopes operate in higher energies. They are, thus, concentrated in the right hand side of the plot (i.e., 
HESS/VERITAS, HAWC, MAGIC and the upcoming CTA South). The space-based ones are in the the $\sim 1$ to $\sim 10^{5} \mathrm{MeV}$ energy range (i.e., Fermi-LAT, EGRET, COMPTEL and the concept e-ASTROGAM). In this Section, we describe both of these techniques.

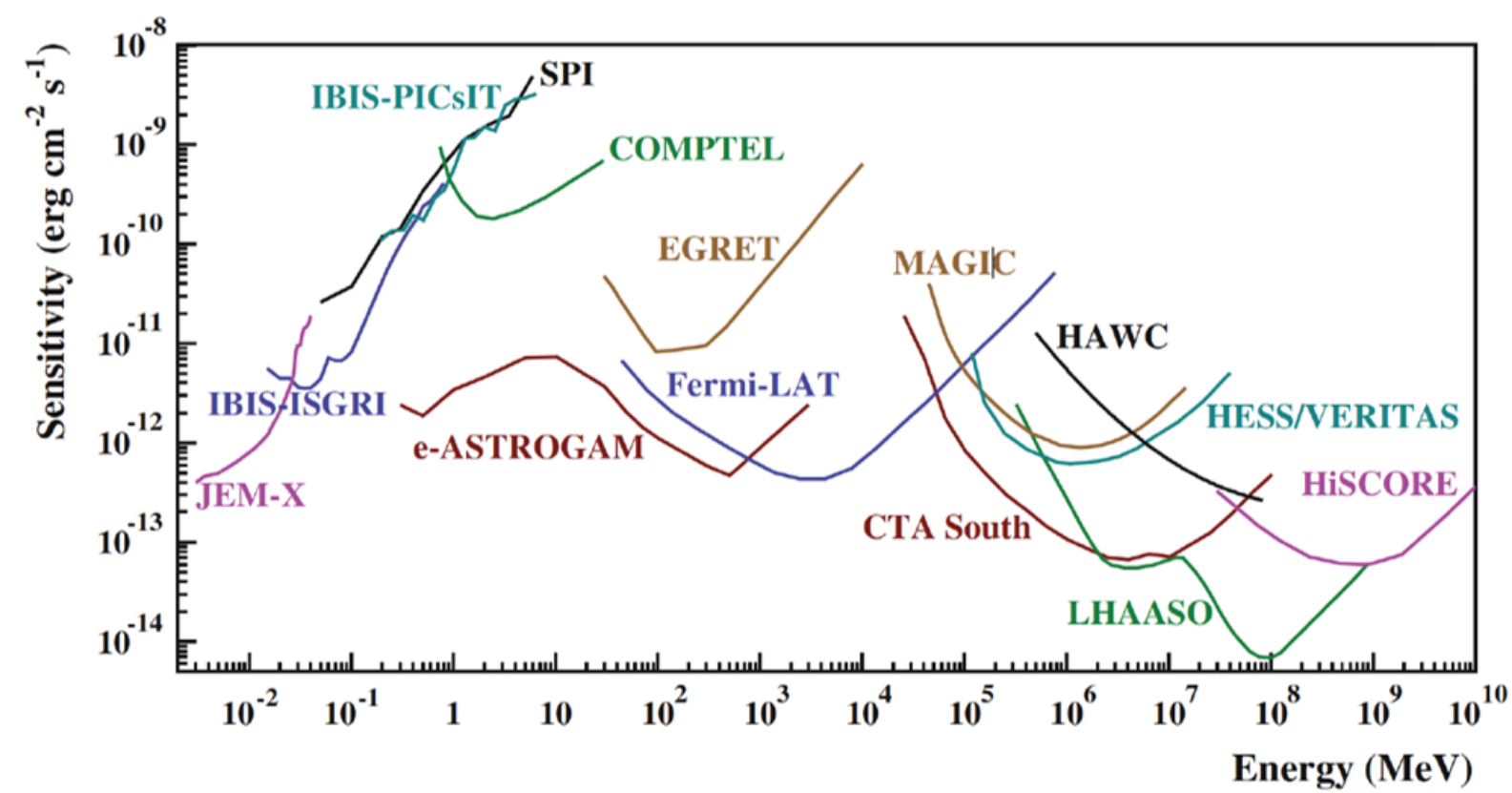

Figure 1.9: Point source continuum differential sensitivity of different X-ray and gamma-ray instruments for a source at high Galactic latitude. Source: de Angelis and Mallamaci (2018). We refer to their work for a discussion on how the the sensitivities were estimated.

\subsubsection{Space-based instruments}

The energy range of space-based gamma-ray telescopes vary between $\sim 300 \mathrm{keV}$ and $\sim 500 \mathrm{GeV}$. The upper boundary is limited by flux because of the small effective area and the scarcity of high energy gamma-ray photons.

The angular resolution of these telescopes is limited by multiple scattering, which increases with the mass of the converter. At the same time, the detector efficiency is a function of mass (denser materials correspond to higher probability of interaction). So, there is a compromise between increasing the effective area and degrading the angular resolution.

The history of gamma-rays space telescopes is plentiful of examples:

- The first generation of such instruments were launched between the end of the 1960s and the beginning of the 1970s: The Third Orbiting Solar Observatory $5^{5}$ (OSO-3) in

\footnotetext{
${ }^{4}$ Not considering the Gamma-ray Burst Monitor instrument on board of Fermi, which is sensitive to photons with energies between $\sim 8 \mathrm{keV}$ and $\sim 40 \mathrm{MeV}$ (Section 1.5)

${ }^{5}$ heasarc.gsfc.nasa.gov/docs/heasarc/missions/oso3.html
} 
1967; the second NASA Small Astronomy Satellite ${ }^{6}(\mathrm{SAS}-2)$ in 1972; and the COS-B in 1975 (Swanenburg et al., 1981).

- The Compton Gamma-ray Observatory] (CGRO) operated from 1991 until 2000. It was comprised of four instruments: the Burst And Transient Source Experiment (BATSE), the Oriented Scintillation Spectrometer Experiment (OSSE), the Imaging Compton Telescope (COMPTEL), and the Energetic Gamma Ray Experiment Telescope (EGRET).

- The INTErnational Gamma-Ray Astrophysics Laboratory ${ }^{8}$ (INTEGRAL, launched in 2002) and The Neil Gehrels Swift Observatory ${ }^{9}$ (launched in 2004) are monitoring the sky in lower energies (going from $100 \mathrm{keV}$ to a few $\mathrm{MeV}$ ).

- The Astro-rivelatore Gamma a Immagini LEggerd ${ }^{10}$ (AGILE) was launched in 2007 and is still in operation.

- Fermi Gamma-ray Space Telescope initiated a new era for space-based observations in 2008. This instrument is described in further details in Section 1.5 ,

- The DArk Matter Particle Explor ${ }^{11}$ (DAMPE) was launched in 2015. It is an instrument of the Chinese Academy of Sciences and searches for Dark Matter signatures.

Proposed MeV-GeV missions e-ASTROGAM (de Angelis et al., 2018) and AMEGO (McEnery et al., 2019) can dramatically improve the observational sensitivity in this energy band.

\subsubsection{Ground-based Cherenkov telescopes}

The ground-based gamma-ray telescopes come in two flavors, depending on how they detect the electromagnetic cascade originated by the interaction of the gamma-rays with the atmosphere.

\footnotetext{
${ }^{6}$ heasarc.gsfc.nasa.gov/docs/sas2/sas2.html

7 heasarc.gsfc.nasa.gov/docs/cgro/

${ }^{8}$ cosmos.esa.int/web/integral

${ }^{9}$ swift.gsfc.nasa.gov

10 agile.rm.iasf.cnr.it/

11 dpnc.unige.ch/dampe/
} 
Instead of using instruments to convert the gamma-ray, they use the atmosphere, greatly increasing the effective area. This allows the detection of very high energy photons - in the $\mathrm{GeV}$ and $\mathrm{TeV}$ range. At the same time, electromagnetic showers originated by lower energy photons are not detectable, which constrains the operation of these instruments.

\subsubsection{The Imaging Air Cherenkov Technique}

The Imaging Air Cherenkov Technique (IACT) detects the Cherenkov radiation from the charged particles of the electromagnetic shower in the atmosphere. Since most of them have a speed larger than light in air, they emit Cherenkov radiation that can be detected by special telescopes on the ground. These instruments have effective area sizes comparable to that of the light pool on ground (i.e. $\sim 10^{4} \mathrm{~m}^{2}$ ).

By observing the air shower with several different detectors spread on the ground, it is possible to reconstruct (with the aid of computational models) the direction and energy of the original gamma-ray. The intensity and area of the image provide an estimate of the energy, while the image orientation is related to the direction. Also, the shape of the image is characteristic of the nature of the events originating them and is used to identify and reject the background from charged particles.

These instruments are installed at high altitudes to facilitate detections. For instance, from a primary photon of $100 \mathrm{GeV}$, about 10 Cherenkov photons per square meter will arrive at the ground in a mountain at $2000 \mathrm{~m}$ above sea level de Angelis and Mallamaci, 2018). A collection area of $100 \mathrm{~m}^{2}$ is therefore sufficient to detect gamma-ray showers. Also, clear and dark nights are necessary for observations since the Cherenkov light is faint.

Among the IACTs, three deserve mention. The Very Energetic Radiation Imaging Telescope Array System² (VERITAS) operates since 2007 in Arizona at the Whipple Observatory. Its observational energy range is between $\sim 100 \mathrm{GeV}$ and $\sim 30 \mathrm{TeV}$. The Major Atmospheric Gamma-ray Imaging Cherenkov Telescopes ${ }^{13}$ (MAGIC) operates since 2004 at the Canary Island of La Palma. The High Energy Stereoscopic System ${ }^{14}$ (H.E.S.S.) started functioning in 2004, in Nabia. Both MAGIC and H.E.S.S. can detect photons with

\footnotetext{
12 veritas.sao.arizona.edu/

13 magic.mpp.mpg.de/

14 mpi-hd.mpg.de/hfm/HESS/
} 
energies as low as $30 \mathrm{GeV}$.

The Cherenkov Telescope Array (CTA, Cherenkov Telescope Array Consortium et al. 2019 ) is planed to start operating in the next few years. It will consist of two IACTs arrays: one in the Southern Hemisphere, in the Atacama Desert in Chile, focused on galactic sources, including a Key Science Project concentrated in the GC; and another in the Northern Hemisphere, on the Spain's Canary Islands, with emphasis on the study of extragalactic objects. CTA will be the most important instrument to study high-energy gamma-rays in the next decade. For instance, it will allow a deep exposure of the GC in energies up $\sim 300 \mathrm{TeV}$, thereby enabling studies in spatial and spectral details which are not possible today, with arc-minute resolution at energies above Fermi's operational range.

\subsubsection{The Extensive Air Showers Technique}

The Extensive Air Showers (EAS) observatories detect the secondary particles formed in the air showers originated by the interaction of a gamma-ray photon with the atmosphere. The shower geometry is reconstructed based on the arrival times, distribution and density of the secondary particles. Showers originated from the interaction of CR with the atmosphere can be rejected based on their geometry and muon content

The most prominent EAS is the High Altitude Water Cherenkov $y^{15}$ (HAWC) that is in operation at $4100 \mathrm{~m}$ above sea level in Mexico. It consists of 300 water-Cherenkov detectors. The secondary particles in the air showers emit Cherenkov radiation when traveling through the water in the detectors. This emission is used to reconstruct the direction and energy of the gamma-ray.

\subsection{Fermi Gamma-ray Space Telescope}

Earth's atmosphere is opaque to gamma-rays (Figure 1.10). One way to deal with this limitation is to detect them from space. Different approaches and instruments are described in Section 1.4 .

The way gamma-rays interact with matter - through Compton scattering, $\mathrm{e}^{-} \mathrm{e}^{+}$pair

\footnotetext{
15 hawc-observatory.org/
} 


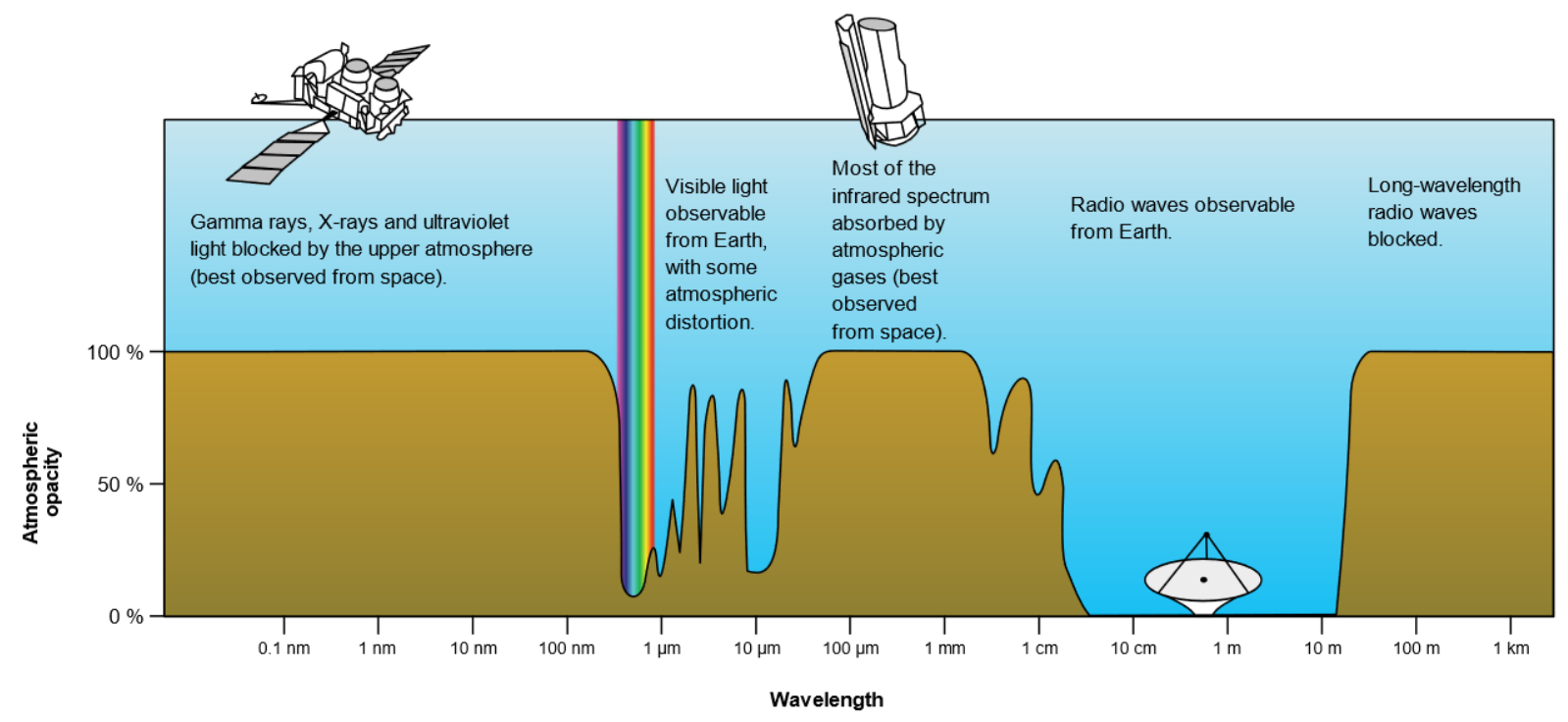

Figure 1.10: A diagram showing Earth's atmospheric opacity as a function of wavelength of the electromagnetic spectrum. Source: NASA (original); vectorized by Mysid / Public domain.

production and photoelectric effect - prevents the use of reflection or refraction to focus them. For this reason, a gamma-ray telescope uses techniques from particle accelerators to detected the byproducts of the photon interaction.

In June $11^{\text {th }}$, 2008, Fermi Gamma-ray Space Telescope (Fermi, Atwood et al. 2009) was launched to an orbit of $\approx 535 \mathrm{~km}$ above sea level. Fermi carries two instruments: the Gamma-ray Burst Monitor (GBM) and the Large Area Telescope (LAT, more details in Section 1.5.1). In Figure 1.11 we show a reproduction of the telescope and its instruments.

The GBM was designed to detect sudden flares of gamma-rays produced by gamma ray bursts. It observes the whole sky (that is not obstructed by Earth). It is sensitive to Xrays and gamma rays with energies between $\sim 8 \mathrm{keV}$ and $\sim 40 \mathrm{MeV}$. After a trigger, GBM calculates preliminary position and spectral information that is transmitted to the ground. A GBM detection can also lead to an autonomous repointing of Fermi to expose the region to Fermi-LAT and proceed with more-detailed data collection. Other observatories can also use GBM's alerts to make opportunistic data collection.

In its regular operation (survey mode) both instruments on board Fermi observe the whole sky every $\approx 3$ hours (equivalent to 2 complete orbits, each one with the telescope's zenith facing opposite hemispheres). This allows for two complementary approaches to gamma-ray astronomy:

- Continuous exposure of the gamma-ray sky, allowing for deeper and sharper images, 


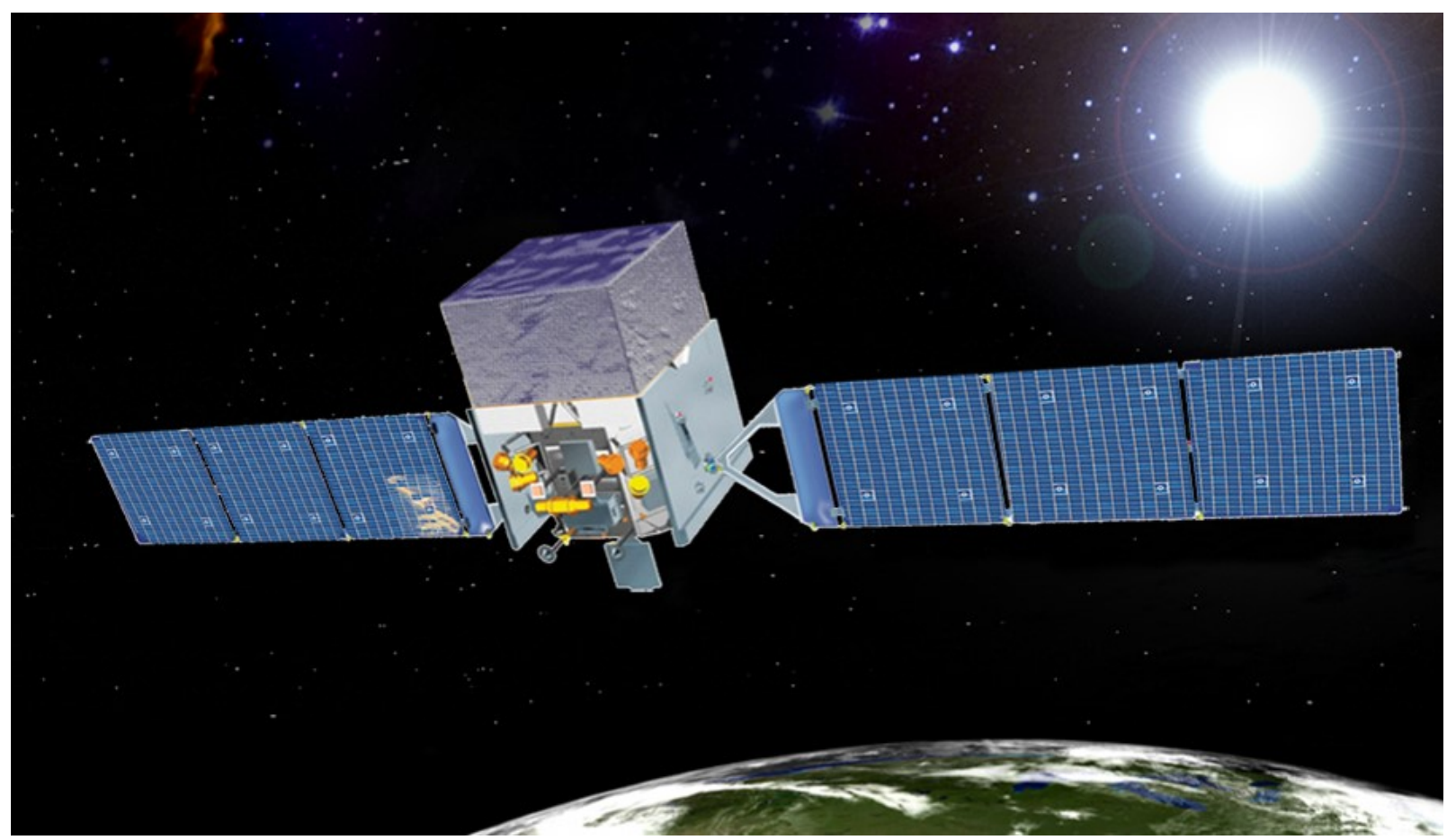

Figure 1.11: An illustration of Fermi in orbit. The cube on the top of the telescope is FermiLAT. The GBM is composed of the yellow instruments at the bottom. Source: NASA (original from: fermi.gsfc.nasa.gov); adapted for this thesis.

but eventually limited by the instruments resolution, even of faint sources;

- Time domain gamma-ray astronomy, enabling variability investigations into time scales going from milliseconds to years.

According to criteria defined by the Fermi-LAT Collaboration, Fermi can also operate in pointed mode observation, when the zenith of the telescope is altered to emphasis observations of selected sources or regions. This happened, for instance, during the whole 2014 year (from December $5^{\text {th }} 2013$ to December $3^{\text {rd }} 2014$ ) to accompany the approximation of the G2 object to Sgr A* (Section 1.3.2).

With more than 12 years of data collection, Fermi has been instrumental in a series of momentous discoveries. Here we describe only three (two that were considered the most important on the occasion of Fermi's $10^{\text {th }}$ anniversary celebration ${ }^{16}$, and one that occurred more recently):

- Fermi Bubbles: they were discussed in Section 1.3.3.4

16 fermi.gsfc.nasa.gov/fermi10/brackets/ 
- Multi-messenger observations from a binary neutron star merger: up until October $16^{\text {th }}$ 2017, the five published astrophysical Gravitational Waves (GW) detection by the LIGO and VIRGO Collaborations were of merging BHs, which are not expected to produce a detectable electromagnetic signal. In August $17^{\text {th }} 2017$, occurred the first GW observation which has been confirmed by non-gravitational means Abbott et al., 2017a). Fermi played an important hole in this detection. As the neutron stars collide, some of the debris blasts away in particle jets moving at nearly the speed of light, producing a brief burst of gamma-rays. They were also detected by GBM and INTEGRAL which helped to constrain the location of the merger and started an intense observing campaign to search for the expected emission at longer wavelengths. The aftermath of this merger was also seen by many dozens of observatories, across the electromagnetic spectrum, marking a significant breakthrough for multi-messenger astronomy (Abbott et al. 2017b).

- Neutrino detection from a flaring blazar: Both gamma rays and neutrinos can be produced from the interaction of cosmic rays (CRs) with nuclear targets, such as molecular clouds (see Section 1.6.2). Neutrinos are not deflected by magnetic fields, nor, because of their small interaction cross-section, are absorbed. On the other hand, they are extremely difficult to detect, because their cross-section are the lowest among elementary particles. Identifying neutrino sources and their association with gamma ray counterparts therefore provides unique insights into the long-standing problem of the CR origin. A potentially compelling evidence has been found on September $22^{\text {nd }}$ 2017, when Fermi-LAT and MAGIC (described in Section 1.4) detected an enhanced gamma-ray emission from the source TXS 0506+056, positionally consistent with a $\sim 300 \mathrm{TeV}$ neutrino, IC170922A, detected by IceCube (The IceCube Collaboration et al., 2018). This suggests that blazars may indeed be one of the long sought sources of very-high-energy CR.

In the next Section, we focus on the main instrument used in this work, the Fermi-LAT.

\subsubsection{The Fermi Large Area Telescope}

Fermi-LAT is the main instrument on board of Fermi. In this Section, we briefly describe its chief characteristics, components, and functioning. For a comprehensive technical 
description of Fermi-LAT, we refer the reader to Atwood et al. (2009).

Fermi-LAT detects gamma-rays in the energy range of $\sim 10 \mathrm{MeV}$ to $\sim$ a few TeV. Its effective area and PSF depend on the photon energy (Section 1.5.3). The spacecraft orbits Earth in $\sim 96$ minutes. It is oriented so that Fermi-LAT always points towards the zenith, therefore the Earth does not block the view. On alternate orbits Fermi rocks to the North and South. This, together with its large field of view ( $\gtrsim 2 \mathrm{sr})$ allows for the whole sky to be surveyed in two orbits.

Since photons in the Fermi-LAT energy range can not be refracted or reflected, the telescope uses the same technology used in high-energy particle detectors. The gammarays interact with the detector through pair production. This process provides a unique signature for gamma-rays, which distinguishes them from charged CRs. It is important since the CR flux is $\sim 10^{5}$ times higher than the gamma-rays flux. Also, the reconstruction of the $\mathrm{e}^{-} \mathrm{e}^{+}$pair trajectories throughout the instrument allows for the determination of the incident gamma-ray direction.

Fermi-LAT was constructed in a 4 x 4 array of identical towers. Each tower comprises a tracker, a calorimeter and a data acquisition module (Figure 1.12). The instrument is covered with a thin anticoincidence shield scintillator. Incoming gamma-rays pass freely through the shield, while charged cosmic rays cause a flash of light, allowing the identification of the relatively rare gamma-rays. Efficient rejection of the charged particle background is essential for Fermi-LAT's functioning. The anticoincidence shield rejects $99.97 \%$ of unwanted signals produced by cosmic rays that enter the telescope.

The tracker is composed of 18 layers of Silicon-strip particle tracking detectors interleaved with Tungsten converter foils. The probability of pair production in photon-matter interactions increases approximately as the square of atomic number of the nearby atom. That is the reason behind using Tungsten (atomic number 74). When a gamma-ray enters Fermi-LAT, it proceeds until interacting with a nucleon from the tracker producing an $\mathrm{e}^{-}$ $\mathrm{e}^{+}$pair. They proceed on, creating ions in the Silicon detectors, allowing for the track of these particles to be precisely measured and used to reconstruct the incident gamma-ray direction. The pair-conversion signature is also used to help reject the CR background.

The design of Fermi-LAT had to attain an equilibrium between the need for thin converters to achieve a good PSF at low energies - which would reduce the amount of scattering of the $\mathrm{e}^{-} \mathrm{e}^{+}$pair inside the instrument, making for a better reconstruction of 


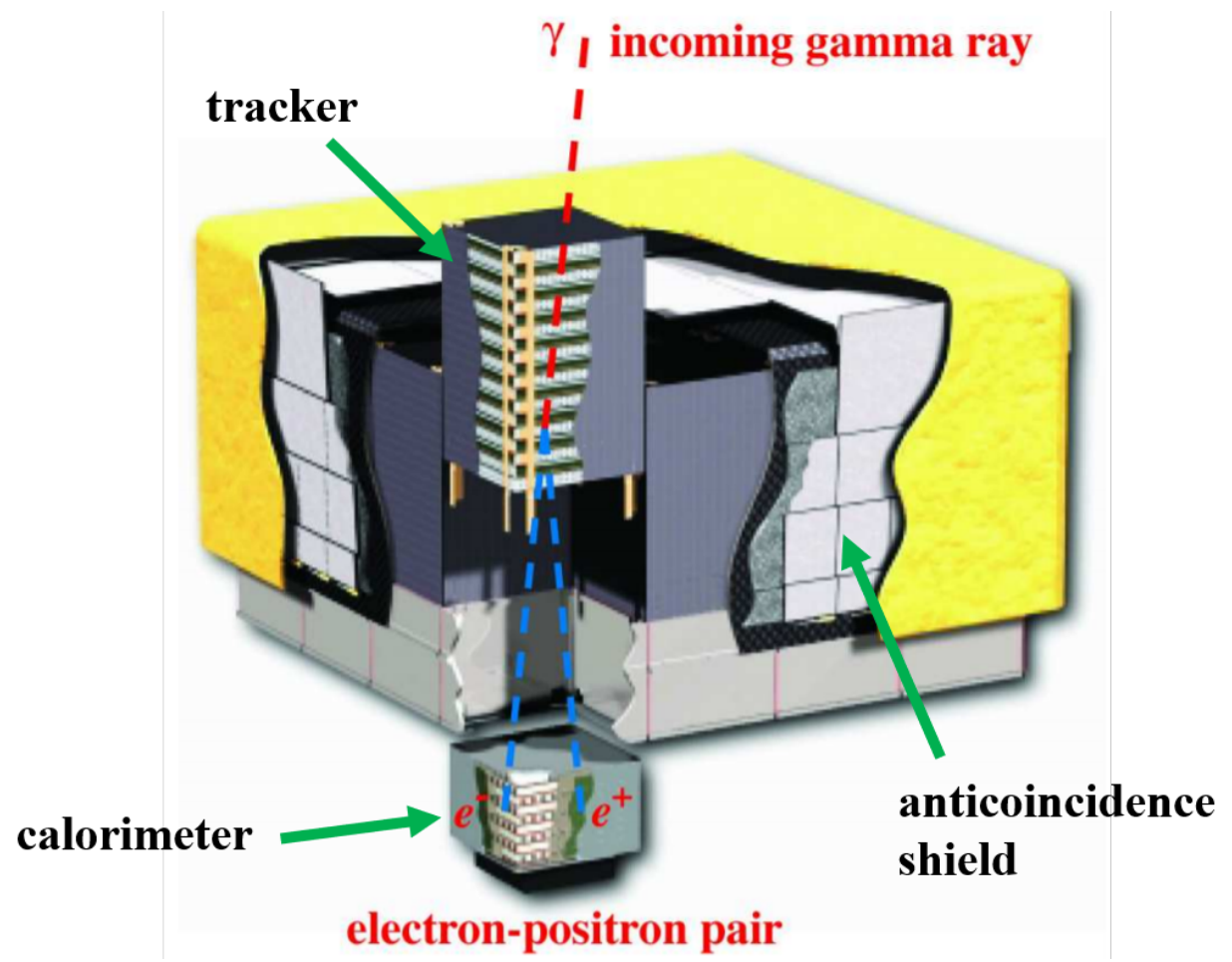

Figure 1.12: An illustration of Fermi-LAT instrument and its constituents. The path of an incoming gamma-ray up to conversion in the instrument is shown, as well as the track of the $\mathrm{e}^{-} \mathrm{e}^{+}$pair until they reach the calorimeter. Source: original from Atwood et al. (2009); adapted for this thesis to label Fermi-LAT's components.

the gamma-ray direction - versus the need for thick converter foils to maximize the effective area, important at high energies - which would increase the likelihood of a conversion. The solution was to separate the tracker into two regions: "front" and "back". The "front" region (composed of the first 12 tracking planes) has thin converters, each 0.03 radiation lengths thick, to optimize the PSF at low energies. The "back" converters (the remaining 4 layers) are $\sim 6$ times thicker, to maximize the effective area at the expense of the angular resolution of photons converting in this region.

After going through the tracker, the $\mathrm{e}^{-} \mathrm{e}^{+}$pair is absorbed by a calorimeter made of Cesium Iodide scintillators (for a total thickness of 10 radiation lengths) which measures the total energy deposited. The material in the calorimeter produces flashes of light whose intensity is proportional to the energies of the incoming particle. The calorimeter can measure the three-dimensional profiles of showers which also helps to reject cosmic rays, since their pattern of energy deposition is different from that of gamma-rays.

The information from the three components (anticoincidence shield, tracker and calorimeter) are combined by a data acquisition system in order to identify when a likely 
gamma-ray has been detected and to decide which information will be sent to the ground for further processing and distribution.

\subsubsection{Fermi-LAT data products}

Any Fermi-LAT data analysis starts with a list of counts that have been identified as resulting from astrophysical photons. It requires information about where the telescope was pointing and what was its observing efficiency. To accomplish this, it is necessary to use two types of data: a file with the photons information and one with the spacecraft information. All these data are available ${ }^{17}$ for the whole scientific community, together with the analysis software ${ }^{18}$ (the Fermitools), without embargoes. In Section 2 we describe in further details how these files and software were used for the analyses presented in this work.

The Fermi-LAT records individual readouts, called events, that are made available by the Fermi-LAT Collaboration in the form of event files (with .fits extension). They contain the reconstructed direction, the reconstructed energy, the moment in time of the detection and quality parameters for each event. There are about $3.6 \times 10^{9}$ events in the Fermi-LAT database detected from the whole sky between August $4^{\text {th }} 2008$ (the beginning of Fermi operation) and September $21^{\text {st }} 2020^{19}$. When only the events with a high probability of being photons (as will be explained below) are considered, this number decreases to around $1.4 \times 10^{9}$.

Fermi-LAT instrument response varies significantly with the photon incident angle (section 1.5.3). So it is necessary to know the position and orientation of Fermi to account for variation in the telescope exposure to the source of interest during its orbit. This information is provided in 30-second intervals by the Fermi-LAT Collaboration through the spacecraft file (also a .fits file). Another reason for this file is that the spacecraft is not collecting data during the whole time. No science data is taken while the telescope is under software maintenance or instrument calibration and while the observatory is transiting the South Atlantic Anomaly (a region over the South Atlantic with a high concentration of charged particles trapped by the Earth's magnetic field configuration).

\footnotetext{
17 fermi.gsfc.nasa.gov/ssc/data/access/

18 fermi.gsfc.nasa.gov/ssc/data/analysis/

19 fermi.gsfc.nasa.gov/cgi-bin/ssc/LAT/LATDataQuery.cgi
} 
The information regarding these moments are provided in the spacecraft file.

A description of every column in the Fermi-LAT data files (both for the event files and the spacecraft file) is available onlin $\AA^{20}$.

Other files, also provided by the Fermi-LAT Collaboration are necessary in the analyses. They include the Instrument Response Functions (IRFs), a catalog of sources (1.5.5) and diffuse emission models 1.5.5.1.

The combination of these files in an analysis is possible with the Fermitools. This process is described in section 2.1 in which we also explain our data selection and preparation.

The likelihood of each Fermi-LAT's detection to be a well-reconstructed gamma-ray is determined by algorithms developed by the Fermi-LAT Collaboration. These quality parameters separate the data into event classes. The categories are hierarchical: the higher probability photon selections are subsets of less restrictive selections. Evidently, the higher probability photon selections have narrower PSF and lower contamination of background events (detections with high likelihood of being contamination from CRs). At the same time, the choice of categories with only probability photon comes with a corresponding drop in statistics. It is, therefore, a trade off that the user has to make. The loosest selection criteria is recommended for short duration events (like gamma-ray bursts, GRBs, with timescales of a few seconds), and timing studies that benefit from increased photon statistics while tolerating a higher background fraction and broader PSF. The cleaner photon selections provide lower background contamination at the expense of lower effective areas. For most of the analysis, like the one presented in this work, an intermediate selection (event class SOURCE) is prescribed.

Fermi-LAT photons are also classified in different event types. They can be categorized according to the location where they converted in the detector (photons converted in the front of the equipment have better angular resolution than those converted in the back), or according to the quality of the reconstructed direction (the photons are divided in quartiles depending on the quality of this reconstruction), or according to the quality of the energy reconstruction (again, in quartiles). In our work, we chose to use the $75 \%$ photons with better reconstructed directions.

In addition to differences in the quality of the data, each subdivision uses a different

\footnotetext{
20 fermi.gsfc.nasa.gov/ssc/data/analysis/documentation/Cicerone/Cicerone_Data/LAT_Data_Columns.html
} 
instrument response function. We explain the impact of this choice on the performance of Fermi-LAT in the next Section.

\subsubsection{Fermi-LAT performance}

The performance of Fermi-LAT is determined by three entities: its hardware, the event reconstruction algorithms and the photon selection (referred as event class and event type, respectively). The IRFs are parametrized representations of the Fermi-LAT instrument performance and are factored into three terms: efficiency in terms of the detector's effective area, resolution as given by the PSF, and energy dispersion. They are used in the analysis process to transform the data collected into meaningful results.

Since Fermi's launch, the Fermi-LAT Collaboration periodically releases updated versions of Fermi's photon dataset and analysis software. This is done to take advantage of improvements in the understanding of the LAT and its orbital environment. The current version at the time of writing is Pass 8 Release 3 version 2 (P8R3_V2, Ackermann et al. 2012a).

The plots in this section were obtained in the Fermi-LAT Performance webpag€ $£^{21}$. They were created with P8R3_V2 for different choices of event class and event type. In Figure 1.13 we show two plots, both considering only event class SOURCE. The one on the top shows how Fermi-LAT's effective area is dependent on the photon energy for photons classified with event types front, back and front + back (indicated as "total" in the plot). The bottom panel shows the effective area as a function of incidence angle $(\theta)$ for $10 \mathrm{GeV}$ photons with event types PSF0, PSF1, PSF2, PSF3 and PSF0+PSF1+PSF2+PSF3 (in which $P S F 0$ represents the $25 \%$ photons with the worst quality of their reconstructed direction, while PSF3 represents the quartile with the best quality of their reconstructed direction).

For energies $<1 \mathrm{GeV}$ and $>1 \mathrm{TeV}$ the effective area decreases. For lower energies, the reason is the smaller probability of pair production in Fermi-LAT's conversion foils. For the higher energies the decrease in effective area happens because the peak of the particle shower can form outside the calorimeter, making the detections not feasible.

In Figure 1.14 we show four plots with the impact of photon energy and incidence angle on Fermi-LAT's PSF. All were created considering only photons with event class

\footnotetext{
${ }^{21}$ slac.stanford.edu/exp/glast/groups/canda/lat_Performance.htm
} 


\section{P8R3_SOURCE_V2 on-axis effective area}

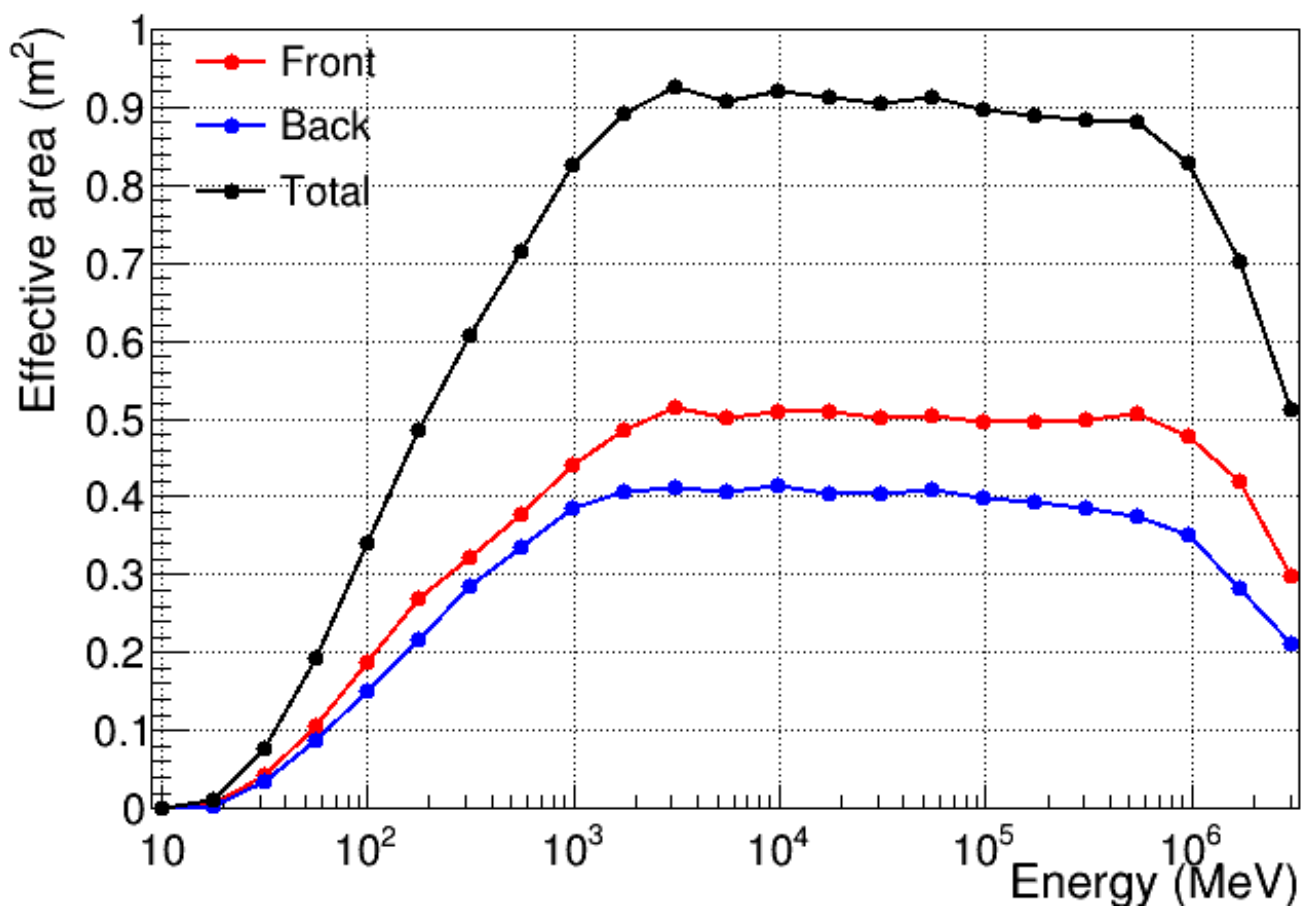

P8R3_SOURCE_V2 effective area at $10 \mathrm{GeV}$, averaged over $\phi$

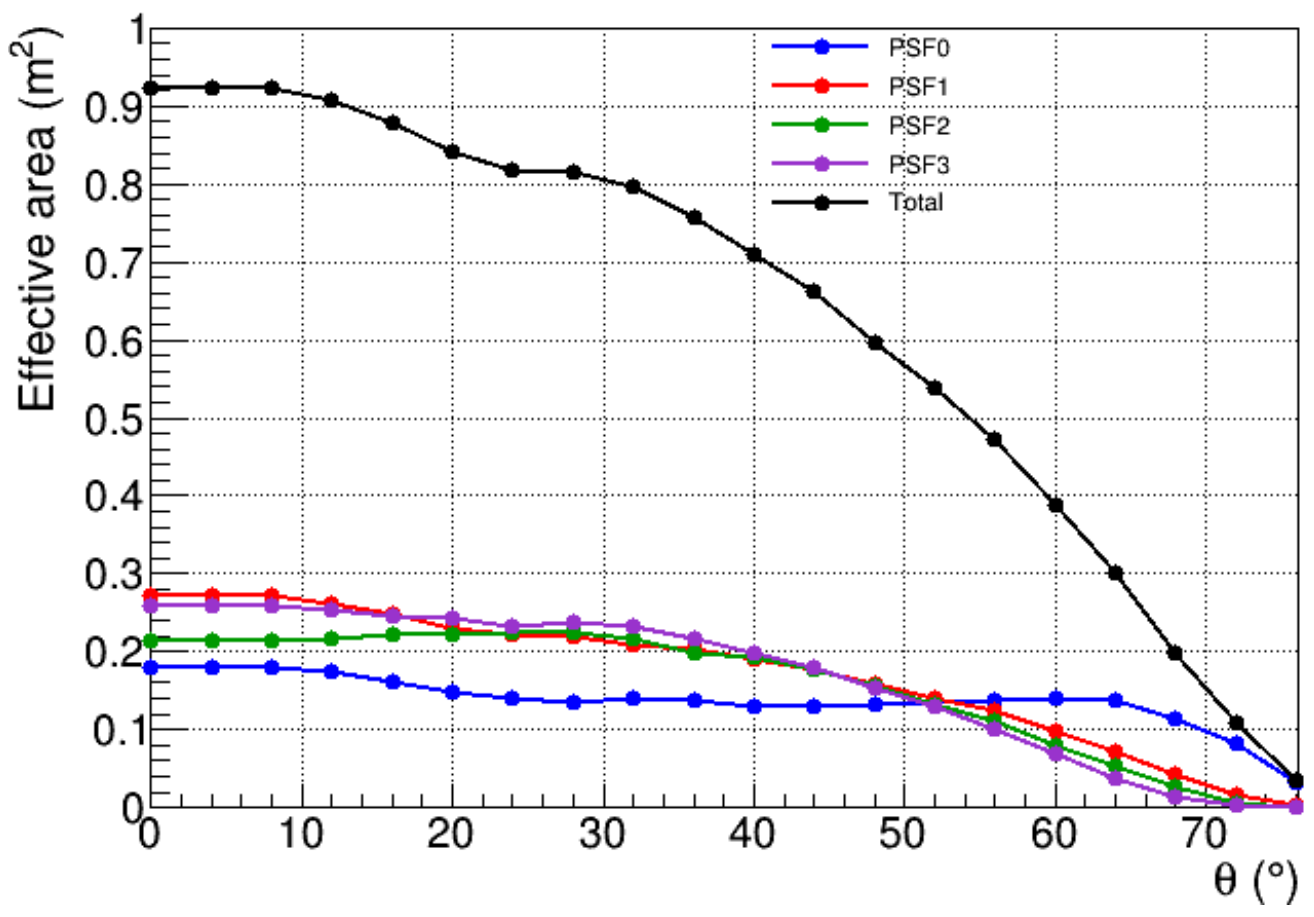

Figure 1.13: Fermi-LAT's effective area as function of energy and incidence angle for photons with event class SOURCE. Top panel: effective area as a function of the photon energy for event types front (red), back (blue) and front + back (black). Bottom panel: effective area as a function of the photon incidence angle for $10 \mathrm{GeV}$ photons with: event types PSF0 (blue), PSF1 (red), PSF2 (green), PSF3 (purple) and PSF0+PSF1+PSF2+PSF3 (black). Source: slac.stanford.edu/exp/glast/groups/canda/lat_Performance.htm. 
SOURCE. The top row shows how Fermi-LAT's PSF depends on the photon energy for different selections of event types: on the left, we show the $68 \%$ and the $95 \%$ confidence level containment angle for photons classified with event types front, back and front + back and on the right the $68 \%$ confidence level containment angle for photons with event types PSF0, PSF1, PSF2, PSF3 and PSF0+PSF1+PSF2+PSF3. For energies $\lesssim 100$ $\mathrm{MeV}$, the uncertainty in the reconstructed direction of a single photon is $\gtrsim 5^{\circ}$. This large value, of course, is diminished with statistics of several photon detections. Because of the broadening of Fermi-LAT's PSF in lower energies, the probability of source confusion is enhanced. Also, since the Galactic diffuse emission is brighter in lower energies, this probability is intensified.

The bottom row in Figure 1.14, we show Fermi-LAT's 68\% confidence level containment angle as a function of incidence angle $(\theta)$ for $10 \mathrm{GeV}$ photons. The impact of the incident angle is mostly significant for high angles. The plots on the right column support our decision to disregard photons classified with event types PSFO in our analysis with the goal of obtaining better resolution on the photons reconstructed direction.

\subsubsection{Caveats and systematics}

The current version of Fermi's photon dataset and analysis software, P8R3_V2, has a few caveats regarding the different event selections and the IRFs. The constant evaluation of all aspects of the data and analysis ensures that the Fermitools are constantly improved and the systematic uncertainties reduced. The Fermi-LAT Collaboration perform constant studies to check the precision and the consistency of the instrument simulations and the instrument response representation provided by the IRFs.

\subsubsection{Effective area}

There are differences between the efficiency of Fermi-LAT instrument in collecting photons (evaluated by the data) and the IRFs predictions. They are translated into systematic uncertainties on the effective area, which is defined as the product of the detector's geometrical area and its efficiency. The effective area is, then, dependent on the source position in relation to the telescope and on the photon energy. These dependencies are considered in the IRFs. 
P8R3_SOURCE_V2 acc. weighted PSF
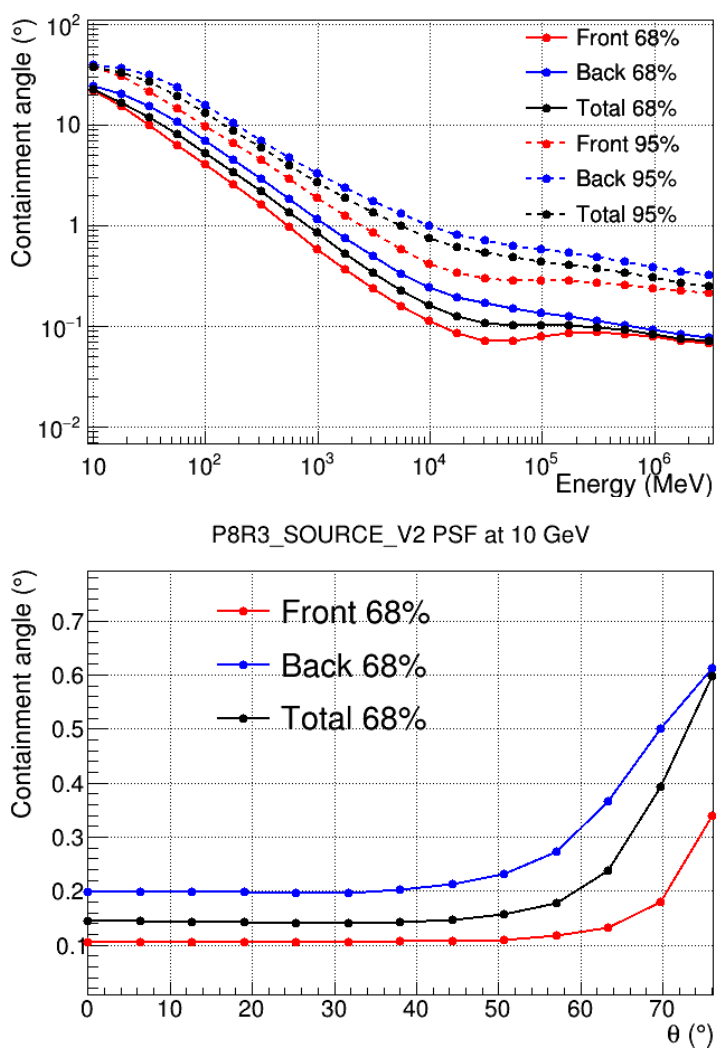

P8R3_SOURCE_V2 acc. weighted PSF

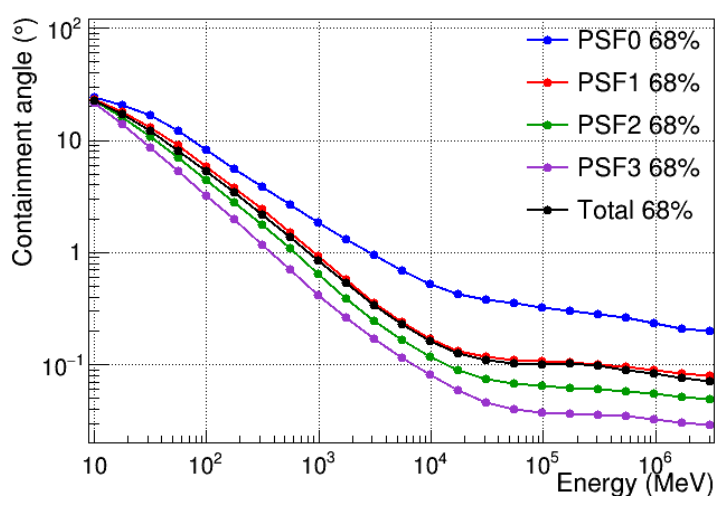

P8R3_SOURCE_V2 PSF at $10 \mathrm{GeV}$

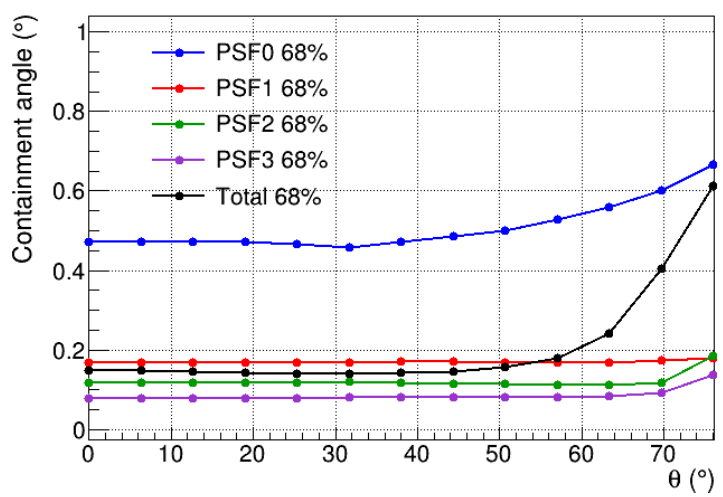

Figure 1.14: Fermi-LAT's PSF as function of energy and incidence angle for photons with event class SOURCE and different choices of event types. Top row, left panel: $68 \%$ and the 95\% confidence level Fermi-LAT's PSF dependency on the photon energy for different for photons classified with event types front (red), back (blue) and front + back (black). Top row, right panel: $68 \%$ confidence level containment angle as a function of energy for different for photons classified with event types PSF0 (blue), PSF1 (red), PSF2 (green), PSF3 (purple) and PSF0+PSF1+PSF2+PSF3 (black). Bottom row: 68\% confidence level PSF as a function of the photon incidence angle $(\theta)$ for $10 \mathrm{GeV}$ photons with the same event types as in the top panels. Source: slac.stanford.edu/exp/glast/groups/canda/lat_Performance.htm. 
The systematic uncertainty on the effective area affects the flux. It is particular important to assess Fermi-LAT's when dealing with light curves (see Section 2.5.2). It is usually less than the statistical uncertainty affecting the light curves points and hence often neglected.

The plot in Figure 1.15 shows how this uncertainty varies with energy for different selection of event types and whether or not energy dispersion is taken into account (see Section 2.3.1 for a discussion on energy dispersion).

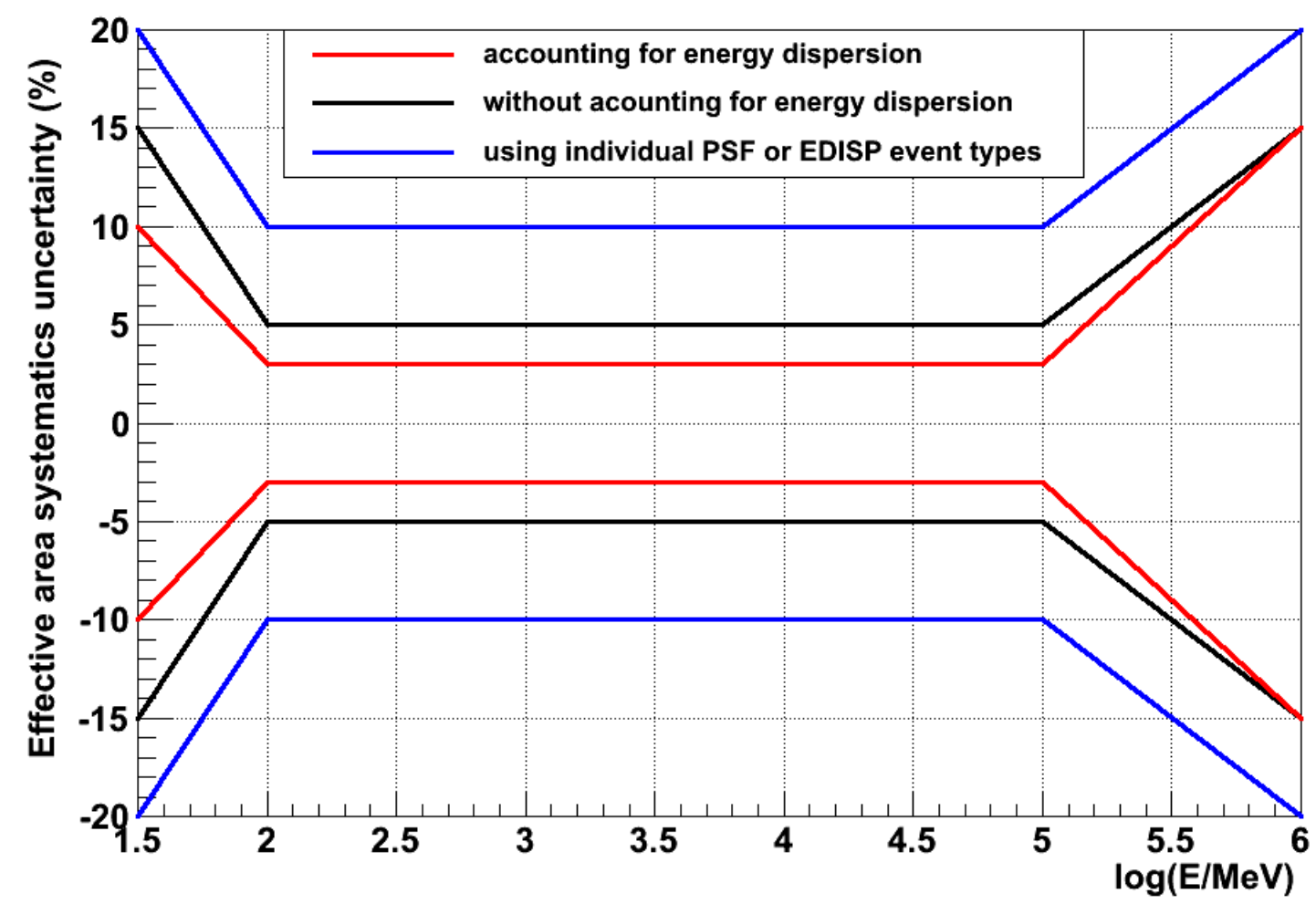

Figure 1.15: The systematic uncertainty band of the Fermi-LAT's effective area for three different configurations: data selection that does not include all events within an event type category and/or with energy dispersion disabled (blue); other data selection with energy dispersion correction disabled (black); and other data selection with with energy dispersion correction enabled (red). Source: fermi.gsfc.nasa.gov/ssc/data/analysis/LAT_caveats.html.

\subsubsection{PSF}

The systematic uncertainties in Fermi-LAT's PSF is quantified in terms of the uncertainty on the $68 \%$ containment radius of a point source. This is performed, for instance, with stacked, high-latitude AGNs. 
The systematic uncertainty on the PSF is shown in Figure 1.16. It is $<5 \%$ in the 100 $\mathrm{MeV}$ to $10 \mathrm{GeV}$ energy range and increases outside this energy band. Above $10 \mathrm{GeV}$ the increase is driven by the limited statistics in the AGNs validation sample. This values apply to all event class and event type selections.

The impact of systematics on the PSF must be evaluated when localizing or assessing a source's extension (see Section 2.4.2).

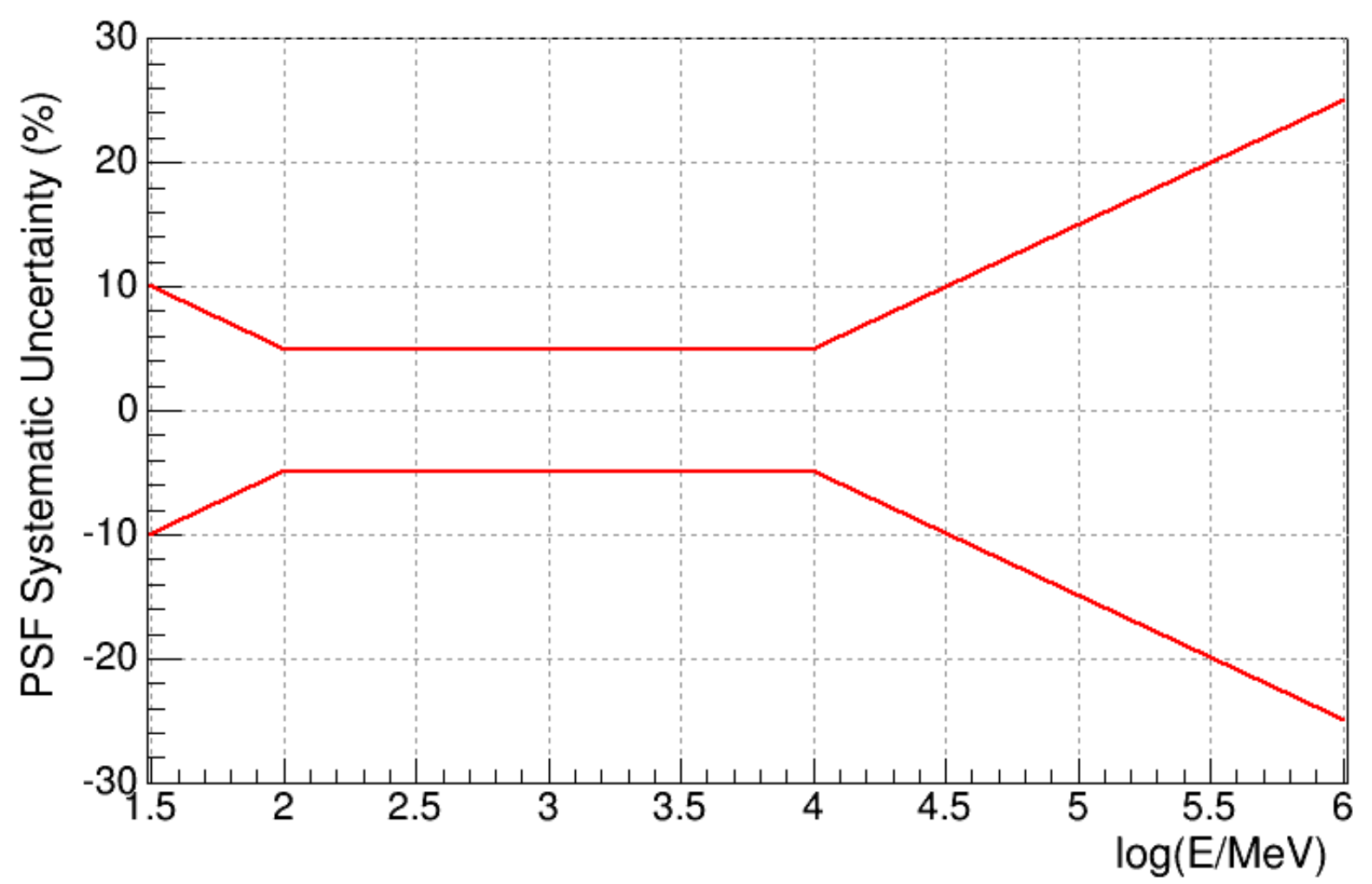

Figure 1.16: The variation of Fermi-LAT's PSF systematic uncertainty with energy. Source: fermi.gsfc.nasa.gov/ssc/data/analysis/LAT_caveats.html.

\subsubsection{The 4FGL Catalog}

As will be further detailed in Section 2.3, to work with Fermi-LAT data, it is necessary to create a model of the gamma-ray emitting sources in the region of the sky being studied. This is a necessity due to (i) the instrument's PSF, (ii) the dependence of Fermi-LAT's effective area on the photon energy and angle of incidence, (iii) the way Fermi operates, and (iv) the nature of the gamma-ray sky backgrounds. An essential resource to create the model is a catalog of known Fermi gamma-ray sources, with their position and spectral model. Since the lauch of Fermi (in June, 2008), the Fermi-LAT Collaboration has been 
releasing catalogs of detected sources. The first catalog (1FGL, Abdo et al. 2010) was released after 1 year of data collection. Other versions of the catalog are 2FGL (Nolan et al. 2012, with 2 years of observations), 3FGL (Acero et al. 2015, with 4 years) and the current version, 4FGL (The Fermi-LAT Collaboration, 2019), with 8 years of survey data and the $50 \mathrm{MeV}-1 \mathrm{TeV}$ range. Recently (June 2020) an incremental version (4FGL-DR2, for Data Release 2, Ballet et al. 2020) of the fourth full catalog of LAT sources, based on 10 years of survey data in the same energy range, was released.

In our work, we used the 3FGL in preliminary analyses that were later updated and expanded with 4FGL data. We did not use 4FGL-DR2 because it was released after all the gamma-ray models we created were already being used and most of the analyses completed. Therefore, in this Section we will describe the 4FGL and its contents. We will briefly mention the upgrades incorporated by the 4FGL-DR2, only to specify that using the 10 year catalog would not significantly improve our analyses and results.

The 4FGL is based on the first eight years of science data from Fermi-LAT in the energy range from $50 \mathrm{MeV}$ to $1 \mathrm{TeV}$. It was created with the most recent version of FermiLAT data, Pass 8 release 3 Version 2 (Atwood et al. 2013) and event class SOURCE (in Section 2.1 we further describe what is the event class selection). The only difference between 4FGL and 4FGL-DR2 data selection regards the time interval covered (from 8 to 10 years).

The 4FGL includes 5064 sources (cf. Figure 1.17) which were detected above $4 \sigma$ significance. The catalog provides their localization and spectral properties. Of these, 75 sources are modeled as spatially extended. A Fermi-LAT source is considered identified with another in different wavelength based on periodic variability for Fermi-LAT-detected pulsars or X-ray binaries, correlated variability at other wavelengths for AGNs, or spatial morphology related to that found in another band for extended sources. A total of 358 sources are considered as identified in 4FGL. As opposed to an identifiction, an association with a source in other wavelength is usually based on the spatial coincidence. This method is unsuitable when dealing with large surveys in the search of a counterpart. In this case, they use a method that retrieve some associations with relatively bright counterparts that were missed by the angular-separation-only method. The 4FGL has 3370 associated sources.

Among the identified and associated sources, more than 3130 are active galaxies of the 
blazar class and 239 are pulsars. Together with the 1336 sources for which they found lower probability of counterparts at other wavelengths, 4FGL totals 5064 sources. The 4FGLDR2 reported 723 new sources (not already in 4FGL), mostly just above the detection threshold. Since in our work we perform a search for new sources (Sections 2.3.1 and 2.3.2 - although not as thoroughly as in 4FGL-DR2 - and the new 4FGL-DR2 sources are very faint, they did not impact significantly in our results.

Each source in 4FGL has a spectral model. Three different functions were used where the normalization, $N_{0}$, was defined at a reference energy, $E_{0}$, chosen such that the error on $N_{0}$ was minimal:

- a subexponentially cutoff power law for all sources identified or associated with a pulsar and with significantly curved spectra:

$$
\frac{d N}{d E}=N_{0}\left(\frac{E}{E_{0}}\right)^{-\Gamma} \exp \left(\alpha\left(E_{0}^{b}-E^{b}\right)\right)
$$

where $\Gamma$ is the low-energy spectral slope, $\alpha$ is the exponential factor and $\beta$ is the exponential index

- a log-normal representation, also referred as 'log-parabola' in this thesi: ${ }^{22}$ for the other sources with significantly curved spectra:

$$
\frac{d N}{d E}=N_{0}\left(\frac{E}{E_{0}}\right)^{-\alpha-\beta \log \left(E / E_{0}\right)}
$$

where $\alpha$ is the spectral slope at $E_{0}$ and $\beta$ gives the curvature of the spectrum

- a simple power-law for all sources not significantly curved:

$$
\frac{d N}{d E}=N_{0}\left(\frac{E}{E_{0}}\right)^{-\Gamma}
$$

where $\Gamma$ is the spectral slope.

For point source spectral models, the units of these functions are $\mathrm{cm}^{-2} \mathrm{~s}^{-1} \mathrm{MeV}^{-1}$.

Also, 4FGL provides a model for two gamma-ray diffuse components: one representing the Galactic emission - associated with hadronic CRs interacting with the molecular gas

\footnotetext{
22 a log-normal distribution has the shape of a parabola in log-log scales, hence it is sometimes referred as 'log-parabola'
} 


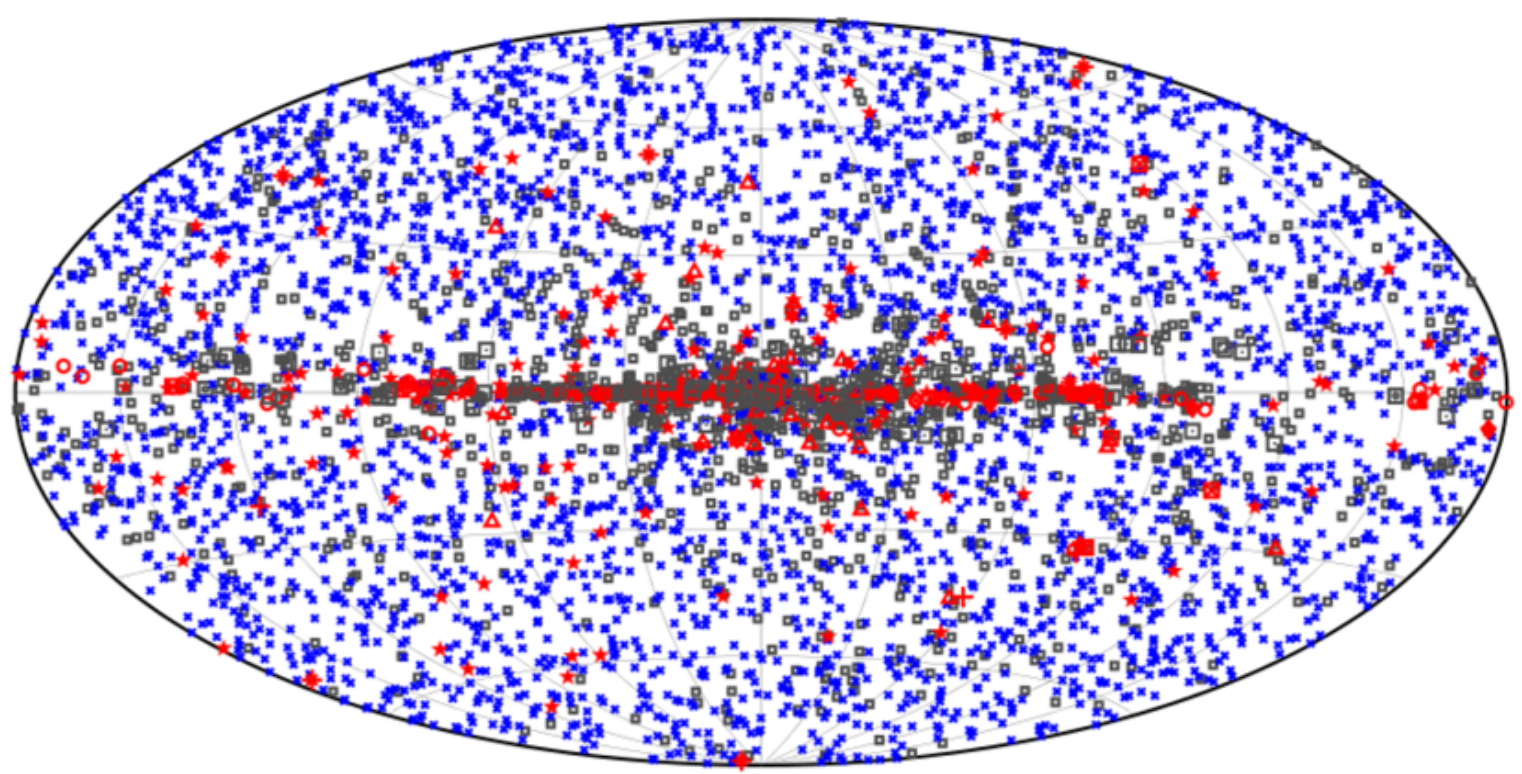

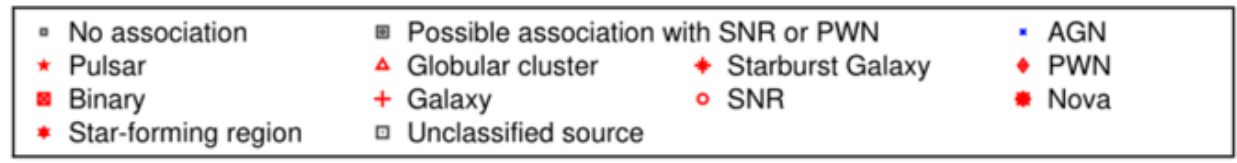

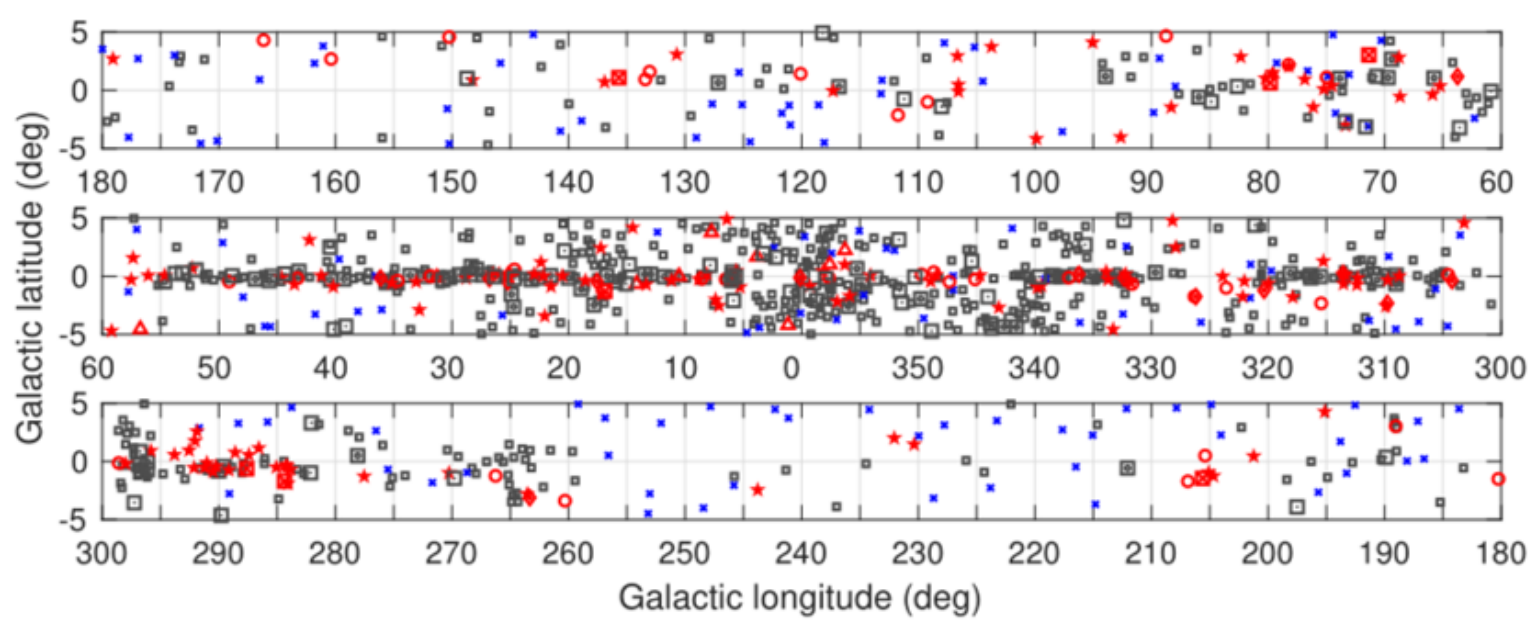

Figure 1.17: Full sky map (top) and blow-up of the Galactic plane split into three longitude bands (bottom) showing sources by source class (no distinction is made between associations and identifications). All AGN classes are plotted with the same blue symbol for simplicity. Other associations to a well-defined class are plotted in red. Unassociated sources are plotted in black. Source: The Fermi-LAT Collaboration $(2019)$. 
content of the Galaxy (Section 1.6.2) - and a isotropic one. They are discussed in Section 1.5.5.1.

\subsubsection{The diffuse models}

In addition to the point and extended sources in $4 \mathrm{FGL}$, there are at least two diffuse components that must be taken into account when dealing with astrophysical gamma-rays in Fermi-LAT's energy range: the Galactic and the isotropic components.

The Galactic diffuse model:

As will be explained in Section 1.6.2, the interaction of hadronic CR with molecular gas emits gamma-rays. Because of the gas distribution in the Galaxy, this gammarays-emitting component is more prominent at low Galactic latitudes. The Fermi-LAT Collaboration released, with 4FGL, the most recent spatial and spectral template of this emission. The development of the model is described in more detail (including illustrations of the templates and residuals) online ${ }^{23}$. Here, we briefly summarize the components of the model and its cataloged spectral model.

This model is based on linear combinations of templates representing components of the Galactic diffuse emission. The templates are the following:

- Gas templates $\left(\mathrm{H}_{I}\right.$ and $\left.\mathrm{CO}\right)$ : This component was developed using spectral line surveys of $\mathrm{H}_{I}$ and $\mathrm{CO}$ (as a tracer of $\mathrm{H}_{2}$ ) to derive the distribution of interstellar gas in 10 Galactocentric rings. Infrared tracers of dust column density were used to correct column densities in directions where the optical depth of HI was either over or underestimated. The model of the diffuse gamma-ray emission was then constructed by fitting the gamma-ray emissivities of the rings in several energy bands to the LAT observations.

- Dark Gas: This is the component of the cold interstellar medium that is not traced in $\mathrm{H}_{I}$ and $\mathrm{CO}$ emission. The existence of this component was established with intercomparison of dust thermal emission maps with $\mathrm{H}_{I}, \mathrm{CO}$, and gamma-ray observations (Grenier et al., 2005). They also split this component into the same 10 Galactocentric rings.

\footnotetext{
${ }^{23}$ fermi.gsfc.nasa.gov/ssc/data/analysis/software/aux/4fgl/Galactic_Diffuse_Emission_Model_for_the_ 4FGL_Catalog_Analysis.pdf
} 
- Inverse Compton on the Interstellar Radiation Field: they used a model from Ackermann et al. (2012b) that was also subdivided in the same 10 Galactocentric rings.

- Large-scale structures: this component represents non-template diffuse gamma-ray emission, primarily Loop I and the Fermi bubbles.

- Cataloged sources: A model that considered the cataloged sources was developed with preliminary versions of 4FGL. This component was used in the Galactic diffuse model creation in order to avoid wrongly attributing cataloged sources' emission to the diffuse model.

- Unresolved Galactic sources: Galactic sources that are too faint to be detected individually undoubtedly contribute to the bright band of interstellar gamma-ray emission at low latitudes.

- Solar and Lunar emission: The Sun and Moon are effectively diffuse sources of gamma-rays when considered in long, integrated data sets. So Solar and Lunar templates were also used.

The Galactic diffuse model was created through a linear combination of these templates. A power-law (Equation 1.3) was used as the spectral model. Because of its large spatial extension, it is the brightest gamma-ray component in the models we created.

The Isotropic diffuse model:

Fermi-LAT data contains a significant background isotropic contribution from extragalactic diffuse gamma-rays, unresolved extragalactic sources, and residual cosmic-ray emission. They are included in the isotropic diffuse model. Although extragalactic unresolved sources are assumed to contribute to the model, it is not strictly a measure of the extragalactic isotropic gamma-ray emission because it includes residual charged particle background.

This model was created over 45 energy bins in the energy range of $30 \mathrm{MeV}$ to $1 \mathrm{TeV}$, from the eight-year data. Emissions close to the Galactic plane $\left(|b|>15^{\circ}\right)$ were not considered to reduce contamination from the bright Galactic diffuse emission. The model was obtained as the residual between the data and the sum of the Galactic diffuse emission model (described above), a preliminary version of the 4FGL catalog and the solar and lunar templates. 
The isotropic diffuse model is provided to the users in a series of tables with the differential flux for each energy bin. There are several versions of this model based on the different data selection used to derive them (i.e., event class and event type, see Section 1.5.1. When fitting a model with Fermi-LAT data, the user can allow a normalization factor to vary the contribution of the isotropic diffuse model.

\subsubsection{The 4FGL J1745.6-2859 source}

The GC is the closest example of a galactic nucleus and a compelling laboratory to investigate the physical processes responsible for accelerating particles to $\mathrm{TeV}$ and $\mathrm{PeV}$ energies. Of the several sources near the GC in 4FGL, 4FGL J1745.6-2859 is the brightest and the closest to $\operatorname{Sgr} \mathrm{A} *$ at a distance of $\sim 0.01^{\circ}$.

Since the beginning of Fermi-LAT's operations, a point source has been observed coinciding with the position of Sgr A*. This source was studied by Chernyakova et al. (2011) with 25 months of Fermi observations (using 1FGL). Later, Malyshev et al. (2015) analyzed the same source using 74 months of data (using 2FGL). In Table 1.2 we show the GC gamma-ray source's position evolution through Fermi-LAT catalogs. We also include the distance do Sgr A* position in radio wavelengths (Reid and Brunthaler, 2004).

Table 1.2 - The position of the central source in the different Fermi-LAT catalogs. We also show the source's distance do Sgr A*'s position in radio wavelengths.

\begin{tabular}{cccc}
\hline \hline $\begin{array}{c}\text { Catalog } \\
\text { version }\end{array}$ & $\begin{array}{c}\text { Source } \\
\text { name }\end{array}$ & $\begin{array}{c}\text { Position }^{1} \\
\left({ }^{\circ}\right)\end{array}$ & $\begin{array}{c}\text { Distance to } \\
\text { Sgr A } *^{2}\left(^{\circ}\right)\end{array}$ \\
\hline 1FGL & 1FGL J1745.6-2900 & $266.419,-29.014$ & 0.035 \\
2FGL & 2FGL J1745.6-2858 & $266.423,-28.979$ & 0.030 \\
3FGL & 3FGL J1745.6-2859c & $266.416,-28.995$ & 0.013 \\
4FGL & 4FGL J1745.6-2859 & $266.415,-28.997$ & 0.011 \\
4FGL-DR2 & 4FGL J1745.6-2859 & $266.415,-28.997$ & 0.011 \\
\hline
\end{tabular}

${ }^{1}$ RA and Dec (in the J2000 epoch)

2 in respect to $\mathrm{Sgr}$ A*'s position in radio wavelengths

It is a point source in all catalogs versions. Its spectral model has changed since the 1FGL and is now cataloged as a log-parabola (Equation 1.2) in both 4FGL and 4FGLDR2. In Figure 1.18 we show its cataloged spectral model, used as the initial template for our models of the Fermi-LAT's gamma-ray sky. 


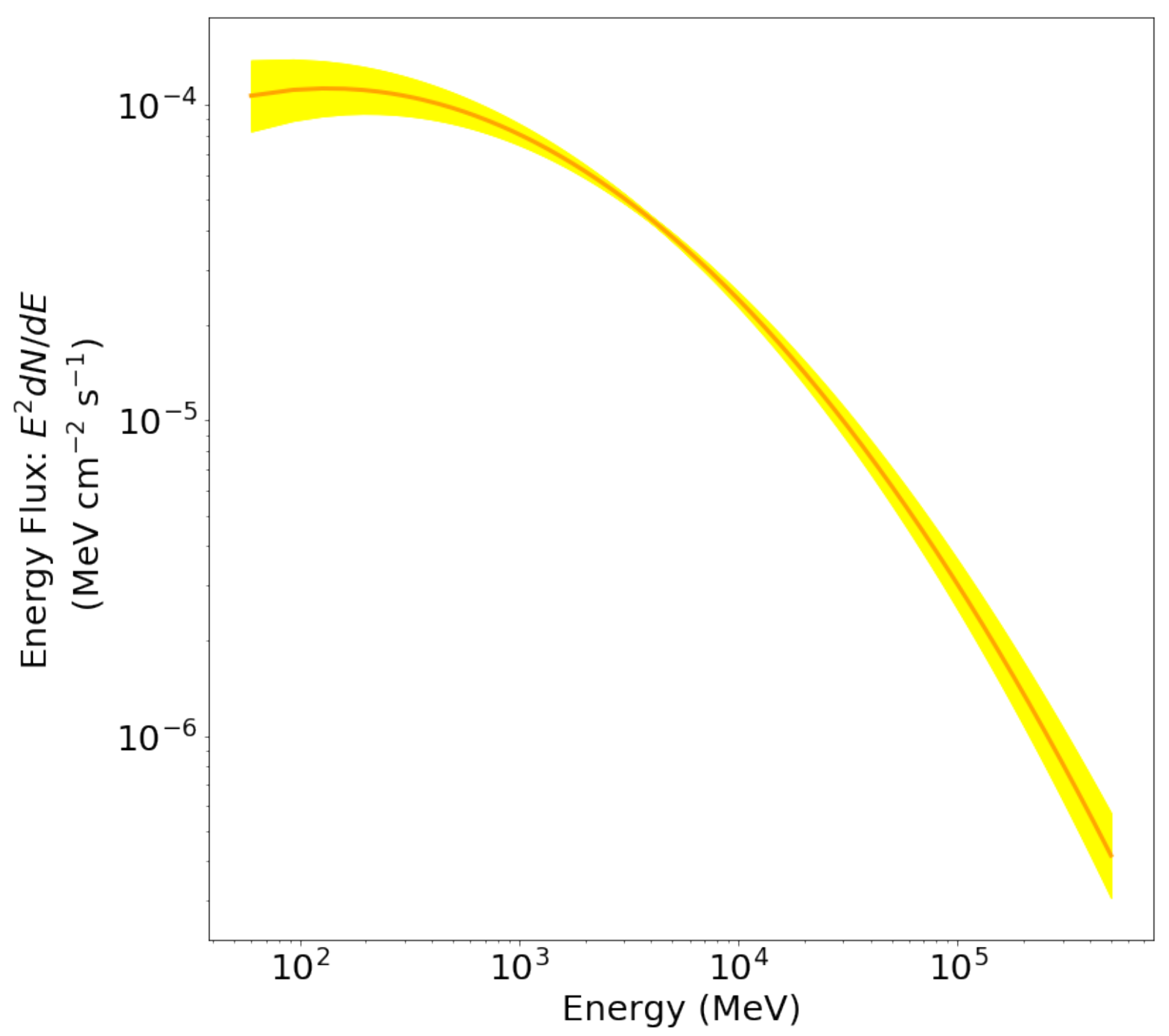

Figure 1.18: The central source's spectral model is cataloged as a log-parabola in 4FGL. Here we show its modeled Energy Flux as a function of energy $\left(E^{2} d N / d E\right)$.

Other than the central source, there are $\sim 200$ 4FGL sources in the central $10^{\circ}$ of the Galaxy. It is the most crowded region of the Fermi-LAT sky and also the brightest one. Since Fermi-LAT's PSF gets to a few degrees in the energy range we are working (Section 1.5.3, specially Figure 1.14, this region is greatly affected by source confusion and considerable care should be taken when modeling it.

\subsection{The production of astrophysical gamma-rays}

In order to understand astrophysical gamma-rays it is fundamental to understand their origin by modeling the electromagnetic emission at the source. The most usual starting 
point is a population of accelerated charged particles in an environment with sufficient magnetic field. The gamma-rays from extragalactic sources are frequently associated to Active Galactic Nucleis (AGNs). Among the Galactic sources, pulsars, pulsar wind nebulae (PWN), supernova remnants (SNR) and binary systems are the most typical sources. Sgr $\mathrm{A} *$ is a natural candidate as a source of $\mathrm{CR}$ responsible for astrophysical gamma-rays. The mechanism responsible for generating gamma-rays is different depending on whether the CRs are leptons or hadrons. In this section, we describe both cases (Sections 1.6.1 and 1.6.2 respectively). Also, we briefly describe, in Section 1.6.3, an aditional scenario in which the gamma-rays are the result of the decay of exotic particles (i.e., dark matter).

\subsubsection{Leptonic emission}

Relativistic charged particles accelerated by magnetic fields emit synchrotron radiation. Since the radiated energy is proportional to $m^{-4}$, where $m$ is the particle mass, this process is much more important for leptons than for hadrons. Typically, the synchrotron photons do not reach to the gamma-ray energy range. Nevertheless, this process is important to undestand leptonic gamma-ray production because leptons can also up scatter low energy photons, transferring part of their kinectic energy to the photons (IC scattering).

Both synchrotron and IC can take place in some astrophysical environments. In particular, ultra-relativistic electrons can generate photons with energies up to the X-ray band via synchrotron. These photons, then, are IC upscattered by the same population of electrons up to the gamma-ray energy band. This process is called Synchrotron Self Compton (SSC) (Dermer and Menon, 2009).

\subsubsection{Hadronic emission}

The interaction of accelerated hadrons with the astrophysical environment can also generate gamma-rays. The collision of a proton with a target nucleon (in a molecular cloud, for instance) initiates a hadron cascade. Almost the same number of $\pi^{+}, \pi^{-}$and $\pi^{0}$ are produced. The $\pi^{0}$ decays almost immediately in two gamma-rays photons, each with close to half the energy of the $\pi^{0}$. The hadronic scenario that is relevant in the context of this thesis is schematically represented by Equations 1.4 and 1.5 .

$$
p+p \rightarrow p+p+\pi^{0}
$$




$$
\pi^{0} \rightarrow 2 \gamma
$$

Each gamma-ray photon depicted in Equation 1.5 has an energy of $m_{\pi^{0}} c^{2}=67.5 \mathrm{MeV}$ in the rest frame of the $\pi^{0}$, where $m_{\pi^{0}}$ is the rest mass of the $\pi^{0}$ (of $\approx 135 \mathrm{MeV}$ ) and $c$ is the light speed. The gamma-ray number spectrum, thus, is symmetric about $67.5 \mathrm{MeV}$ in a $\log -\log$ plot. The SED of this radiation, in the usual $E^{2} d N / d E$ representation, has a distinct bell-type shape, rising steeply in energies below a few hundreds $\mathrm{MeV}$ Stecker, 1971). This spectral feature is often referred as the "pion-decay bump" and is used to identify hadronic astrophysical gamma-ray emission.

The hadronic gamma-rays production is always accompanied by the production of neutrinos. While the $\pi^{0}$ decays almost immediately into two gamma-rays photons, neutrinos are generated as a result of the charged pions decay.

Hadronic gamma-ray emission is the main contribution for the diffuse gamma-ray flux associated to the Galactic disk. The gamma-ray flux with a hadronic origin is thus a function of the $\mathrm{CR}$ and the target gas densities. This process is also relevant in RIAFs. Since ions hardly radiate, they are heated to very high temperatures, resulting in protonproton collisions that result in gamma-ray photons (Mahadevan et al., 1997; Oka and Manmoto, 2003; de Menezes et al., 2020).

\subsubsection{Dark matter self annihilation}

Gama-rays might also be produced as the result of the decay or annihilation of exotic particles. The primary candidate, among several, for dark matter (DM) is elementary particle, in particular, weakly interacting massive particles (WIMPs). The gamma-ray flux expected as the result of these particles sef-annihilation depends quadratically on the DM density along the line of sight (de Angelis and Mallamaci, 2018). This motivates searches on targets where one expects DM density enhancements, like the GC.

\subsection{Publication resulting from this work}

We intend to report the results of this work in two articles, both with this $\mathrm{PhD}$ candidate as first author: 
- Fermi LAT observations of Sagittarius A*: I - Imaging Analysis

- Fermi LAT observations of Sagittarius A*: II - $\gamma$-ray SED and Variability

The first article of this list describes imaging analysis of the source 4FGL J1745.6-2859 across four energy bands from which we obtained its position as a function of energy. We also evaluated its "energetics" in the same energy bands. These results allowed us to rule out several potential candidates for the nature of the point gamma-ray flux from the GC. Our observations favor a cosmic ray origin either from protons, electrons or both, accelerated by - or in the vicinity of - the SMBH. This article was submitted in August $19^{\text {th }} 2020$ to The Astrophysical Journal. The PhD candidate is the first author and leader of this research with their advisor Prof. Dr. Rodrigo Nemmen as a coauthor. In Appendix A we reproduce the draft version of the article as submitted to The Astrophysical Journal. The work reported in this article is reproduced in this thesis (with several additional details) in Sections: 2.2, 2.4, 3.1, 3.2 and 4.1.

The second article of the list is still in preparation. In this work we plan on reporting 4FGL J1745.6-2859 SED and light curve. The goal is to constrain the physical processes responsible with the emission. Several models found in the literature for Sgr A* gamma-ray emission will be compared with the results. Also for this project, the $\mathrm{PhD}$ candidate will be the first author with their advisor as a coauthor. The target is to submit this article to The Astrophysical Journal in the second semester of 2021. Most of the analysis and results that will compose this article are already described in this thesis, in Sections 2.5. $2.6,3.3,3.4,4.2$ and 4.3 .

In Appendix $\mathrm{B}$, we report the participation of the $\mathrm{PhD}$ candidate in the publication of three additional articles. They are not immediately related to the subject of this thesis and are included only for the sake of completeness. 
Chapter 2

\section{Data and Methods}

In this section we discuss the data and the methods used in this work. We start describing how to create a model of the gamma-ray sky with Fermi observations. Then, we explain how we obtained the GC gamma-ray point source position, flux variability and SED with the Fermitools - a suite of data analysis software provided by the FermiLAT Collaboration - and Fermipy (Wood et al., 2017) — a Python package that facilitates analysis of data from Fermi-LAT.

\subsection{Data}

The Fermi-LAT data are available in the form of event files (with .fits extension), with the reconstructed direction and energy of the detection and quality parameters for each event. The Fermi-LAT instrument response varies significantly with the photon incident angle, so it is necessary to know the position and orientation of the satellite to account for variation in exposure to the source of interest during its orbit. This information is provided at 30-second intervals in the spacecraft file, which is also a .fits file.

The combination of the photon and spacecraft files is possible with the Fermitools.

Throughout this work we used Fermitools with the aid of Fermipy to deal with Fermi data and analysis.

The first step is to make cuts on the raw data based on our selection of: energy range, time range, position (RA, Dec), size of the region of interest (RoI), event class, event types and maximum zenith angle.

These cuts are performed using the tool gtselect available in Fermitools.

In the following paragraphs we explain each item of this list and our choices for them. 
We start by describing our energy selection cuts.

With the goals of taking advantage of the better Fermi-LAT's PSF at higher energies (section 1.5.3) and also to evaluate the impact of photon energy on the source position, we divided our analysis into four energy bands that were chosen as explained in section 2.1.3.

- $60-300 \mathrm{MeV}$

- $300 \mathrm{MeV}-3 \mathrm{GeV}$

- $3-10 \mathrm{GeV}$

- $10-500 \mathrm{GeV}$

The three highest energy bands analysis are based in an analysis that we call "Universal Model". It was performed with energies between $100 \mathrm{MeV}$ and $500 \mathrm{GeV}$ and later split in tighter energy bands. The lowest energy band used a custom model created from scratch. We explain the modeling process in Section 2.3. Figure 2.1 shows how we split the analysis in different energy bands.

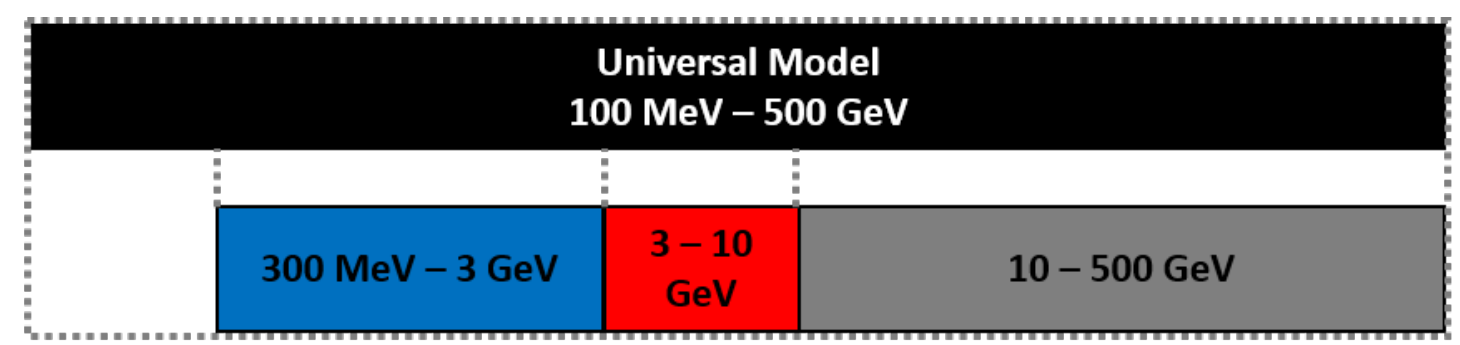

Custom Model

$60-300 \mathrm{MeV}$

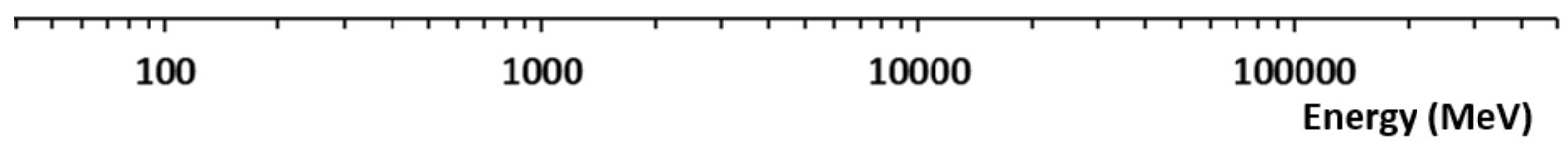

Figure 2.1: We divided our analysis in four energy bands $(60-300 \mathrm{MeV}, 300 \mathrm{MeV}-3 \mathrm{GeV}$, 3-10 GeV and 10-500 GeV). The three highest energy bands models were created based in an analysis that considered energies between $100 \mathrm{MeV}-500 \mathrm{GeV}$. This is our Universal Model which was also used to create LCs and a SED for 4FGL J1745.6-2859. The lowest energy band was studied with a custom model.

To work with Fermi data, it is necessary to create a model for the gamma-ray emitting sources in the RoI. This process will be further described in Section 2.3. Here we focus on the data selection and preparation before the modeling starts. 


\subsubsection{Data selection: Universal Model}

For our Universal Model we worked with photons detected with energies between 100 $\mathrm{MeV}$ and $500 \mathrm{GeV}$. We chose events detected since the start of Fermi-LAToperations (in August $4^{\text {th }}$ 2008, 15:43:36 UTC) until December $1^{\text {st }}$ 2019, 00:00:00 UTC, adding up to $\sim 11.3$ years of data collection.

We considered photons detected inside a square with $20^{\circ}$ side centered in the cataloged point source 4FGL J1745.6-2859 that is coincident with the position of Sgr A*, and rotated $\sim 58^{\circ} .6$ to the East in Galactic coordinates. This large area-when compared with usual astrophysical observations, specially in longer wavelenghts - is necessary to account for Fermi's large PSF (section 1.5.3) and the complexity of the gamma-ray sky in the direction of the GC (section 1.2.1).

Gamma-rays can also be generated by cosmic-ray interaction with the Earth's atmosphere. For this reason, the Earth limb is a strong source of background in Fermi-LAT's data. The maximum zenith angle selection was designed to exclude from the analysis time periods in which the Earth's limb is too close to the RoI. The $90^{\circ}$ recommended 11 value for analysis similar to ours was adopted. This value is sufficient to provide a buffer between the RoI and the Earth's limb (that lies at a zenith angle of $113^{\circ}$ ). In the next step of the data selection process, we will use the gtmktime tool to remove any time period in which the RoI overlaps this buffer region.

The Fermi-LAT events (available through the event files) are classified based on their photon probability and the quality of their reconstruction (section 1.5.2). This separates the photons in different event class, each with its own set of instrument response function. In this work, we used the recommended event class for the characteristics of our analysis, i.e. photons classified as SOURCE. Fermi-LAT photons are also classified in different event types. In this work we chose to consider only the $75 \%$ photons with better reconstructed direction (event types PSF1, PSF2 and PSF3). This was done in order to minimize the risk of source confusion, since the GC is a region crowded with Fermi sources, but without compromising the amount of photons available for the analysis.

The Fermi-LAT response functions are dependent of the angle between the source and the telescope axis. It changes as the satellite orbits Earth and/or its axis is moved to make

\footnotetext{
${ }^{1}$ fermi.gsfc.nasa.gov/ssc/data/analysis/documentation/Cicerone/Cicerone_Data_Exploration/Data_preparation.html
} 
some specific opportunistic observation (to focus on a gamma-ray burst, for instance). Thus, the number of photons detected from a source depends on the amount of time it spends at different inclination angles during the data collection. Also, the detection of photons will depend on the accumulated time during which Fermi-LATis actively taking data, the "livetime". Using the tool gtmktime is a necessary step for this purpose. This tool reads the spacecraft file and creates a set of Good Time Intervals (GTI). A GTI is a time range in which the data, based on the pointing and livetime history of Fermi-LAT, can be considered valid: periods when the instrument was collecting data over the selected time range and the RoI was not close to Earth's limb (based on the maximum zenith angle criteria). Only after removing the time periods when the data quality is not considered good that the exposure (effective area multiplied by livetime) of the RoI is assessed.

After all these steps, we have a list of detection events that satisfy our selection criteria available to work with. They will be now be addressed as 'counts'.

Then, the tool gtltcube computes the livetime as a function of inclination and location on the sky for a specified observation period.

It is interesting to create an exposure map with the total exposure in every position of the RoI during the analysis period. This makes the data processing faster and less computational demanding. The effective area of Fermi-LAT, as explained in Section 1.5.3, is a function of the incident photon energy. For this reason, the exposure map also depends on the energy. The counts produced by a source at any given position of the RoI is the integral of the source flux and the exposure map at that position. The exposure map is particularly important to account for extended sources such as galdiff and isodiff.

\subsubsection{Data selection: low energy custom model}

The low energy (60-300 MeV) model was created totally independent from the Universal Model. Since we generated it a few months before the Universal Model, its time range is smaller, going from August $4^{\text {th }} 2008,15: 43: 36$ UTC to February $16^{\text {th }}$ 2019, 00:00:00 UTC, adding up to $\sim 10.8$ years.

We chose to work with a larger RoI- a square with $30^{\circ} \times 30^{\circ}$ sides centered on the point source 4FGL J1745.6-2859, and with the same orientation used for the Universal Modelbecause Fermi-LAT's PSF is larger at lower energies. Again, we used the recommended ${ }^{2}$

\footnotetext{
${ }^{2}$ fermi.gsfc.nasa.gov/ssc/data/analysis/documentation/Cicerone/Cicerone_Data_Exploration/Data_preparation.html
} 
value of $90^{\circ}$ for the maximum zenith angle cut.

The other data selection choices were the same as in the Universal Model, i.e. event class SOURCE and event types PSF1, PSF2 and PSF3.

Now that the data is selected and prepared, we move on to create the models of the gamma-ray sky necessary to deal with the Fermi data. Before we describe the modeling process per se we will explain how the energy bands we are using were selected (Section 2.1.3 and, then, we will briefly describe the statistical tool used in this step (Section 2.2).

\subsubsection{Energy boundaries}

The choice of the energy bands we used in this work (Figure 2.1) was decided semiempirically. We wanted bands with enough counts to obtain statistically significant results in the analyses. We used 4FGL J1745.6-2859's spectral model (Figure 1.18) to split the total predicted number of photons between $60 \mathrm{MeV}$ and $500 \mathrm{GeV}$ into 4 energy bands with more or less the same number of counts. To do that, we multiplied Equation 1.2 by $E$ (the energy) and integrated in respect with $E$ using several different integration limits. Since Equation 1.2 gives results in therms of an area and the instrument effective area is dependent on the photon energy (Figure 1.16), we knew the results we got were approximations. After obtaining approximated results for the bands frontiers, we chose the closest round values. Them, we performed the data selection steps (Section 2.1) with the obtained energy boundaries and assessed the number of events in each energy band to check if the four of them had about the same number of counts. We had to expand and reduce some of the bands but managed to find limits that granted bands with the desired characteristics.

\subsection{Maximum likelihood modeling}

We use the maximum likelihood technique to find the best fit model to the data. The likelihood is the probability of obtaining the data given a specific input model. In our case, the model consists of the gamma-ray sources distribution on the sky, including their spectra and position. To fit a model, we vary these parameters until the likelihood is maximized.

An underlying hypothesis behind the likelihood fitting is that we suppose that the 
model with the highest probability to explain the data is, indeed, the model closest to the reality beneath the observations. That is not necessarily true, but it represents our best understanding of the underlying reality we are trying to study.

To fit a model to Fermi-LAT's data, the four steps listed below are necessary. We already explained the first three. So, we will just briefly mention them here and address the reader to the Sections where they were further discussed. The fourth item of the list is the one we are interested now.

1. Data selection: This step was explained in Section 2.1. In short, we take data from a spatial region centered in the source of interest to account for nearby sources' emission (because of Fermi-LAT's large PSF and the complexity of the region).

2. Model selection: As mentioned, the model includes the position and spectra of the sources in the RoI. Also, it includes a model of the diffuse emission. The Fermi-LAT Collaboration provides a catalog with these sources and also models for the diffuse components (See Section 1.5.5).

3. Precomputing important quantities: During the model fitting the parameters are varied several times until the maximization of the likelihood. This is a computationally intensive process. To speed it up the livetime and the exposure map (presented in Section 2.1) are previously calculated.

4. Performing the fit: The actual fitting process searches for the combination of parameters that maximize the likelihood of the model explaining the data. Several parameters are fit simultaneously in an iterative fashion. The result of the fitting process is a set of best-fitting parameters and their uncertainties.

\subsubsection{The likelihood function}

Every count in the Fermi-LAT data is characterized by many different variables such as direction, energy, time of detection, part of the telescope in which it was converted, etc.. For analysis in which the number of counts is large (typically, every analysis that take into consideration extended time periods and/or sources that are not extremely faint) the time to calculate the likelihood becomes prohibitive. For this reason, before performing the fit, the data is binned according to the counts' characteristics. Given the number of 
variables and the reduced number of gamma-ray photons that are detected by Fermi-LAT (see Section 1.5.2) when the data is binned, each bin will contain a small number of counts. The observed number of counts in each bin follows a Poisson distribution and can not be approximated by a Gaussian distribution.

For a Poisson distribution, the probability of a number of counts being detected in a given pixel is:

$$
p_{i}=\frac{m_{i}^{n_{i}} e^{-m_{i}}}{n_{i} !}
$$

where $m_{i}$ is the expected number of counts predicted by the model in pixel $i$, and $n_{i}$ is the observed counts in that pixel.

The likelihood $\mathcal{L}$ is the product of the probabilities for every pixel:

$$
\mathcal{L}=\prod_{i} p_{i}=\prod_{i} \frac{m_{i}^{n_{i}} e^{-m_{i}}}{n_{i} !}
$$

We can factor this product into two therms:

$$
\mathcal{L}=\prod_{i} e^{-m_{i}} \prod_{i} \frac{m_{i}^{n_{i}}}{n_{i} !}
$$

We can calculate the first therm as:

$$
\prod_{i} e^{-m_{i}}=e^{-m_{1}} \times e^{-m_{2}} \times \ldots \times e^{-m_{i}}=e^{\sum_{i}-m_{i}}=e^{-N_{\text {pred }}}
$$

where $N_{\text {Pred }}$ is the total number of counts predicted by the model. This therm is independent of the data. The second therm of Equation 2.3 does depend on the data $\left(n_{i}\right)$. Using the result of Equation 2.4 in Equation 2.3, we get:

$$
\mathcal{L}=e^{-N_{\text {pred }}} \prod_{i} \frac{m_{i}^{n_{i}}}{n_{i} !} .
$$

This is the appropriate likelihood function form for a binned analysis.

We are interested in the set of parameters that maximize the likelihood, displayed in Equation 2.5. Because logarithms are strictly increasing functions, maximizing the likelihood $(\mathcal{L})$ is equivalent to maximizing the log-likelihood $(\ell)$. But for practical purposes it is usually more convenient to work with the log-likelihood function in maximum likelihood estimation. So, we take the logarithmic transformation of Equation 2.5. 


$$
\log \mathcal{L}=\ell=\log e^{-N_{\text {pred }}}+\log \prod_{i} m_{i}^{n_{i}}+\log \prod_{i} \frac{1}{n_{i} !}
$$

Which gives:

$$
\ell=-N_{\text {pred }}+\sum n_{i} \log m_{i}-\sum \log n_{i} !
$$

The first therm in Equation 2.7 is just the negative of the total number of counts predicted by the model. The third therm is model independent and, hence, neglected. So, the log-likelihood function that is maximized is simply:

$$
\ell=\sum n_{i} \log m_{i}-N_{\text {pred }}
$$

The binning process destroys information because we don't have access to the precise values of the quantities describing a count. So, the smaller the bins the more accurate the likelihood.

For bin sizes infinitesimally small, which means an unbinned analysis, we get to $n_{i}=1$ (or $n_{i}=0$ ). Then, Equation 2.5 is reduced to:

$$
\mathcal{L}_{\text {unbinned }}=e^{-N_{\text {pred }}} \prod_{i} m_{i}
$$

where $i$ is now the number of detected counts.

In this case, $m_{i}$ is calculated using the precise values for each count (instead of an average over a bin). For this reason, the unbinned likelihood is the most accurate.

And, the log-likelihood function, that is maximized for the unbinned analysis, is:

$$
\ell_{\text {unbinned }}=\log m_{i}-N_{\text {pred }}
$$

In our work, due to the amount of data and huge number of sources in the model, we used only the binned likelihood method, described by Equations 2.5 and 2.8 .

\subsubsection{Likelihood model fitting with Fermitools}

The set of parameters that maximizes the likelihood is determined by the fitting process. The maximum is found through in an iterative manner, by calculating $\ell$ for different sets of parameters. Then, its devirative with respect to the parameters is calculated, which 
guides the choice of a new set of parameters by the algorithm that are progressively closer to the set that maximizes the function. These steps are repeated until the change in the function value between iterations is sufficiently small (or the number of iterations reaches a maximum value). During the iterations, the algorithm maps the dependence of the function on the parameters, particularly near the maximum. The uncertainties on the best fit parameters are related to this dependence and, hence, provided as a result of the fitting process.

The Fermitools offer five choices of algorithms, called optimizers. They vary in how rapidly they converge to the function maximum, the amount of computer memory they require, and the accuracy with which they map out the dependence of the function. Fermipy restrict the choice to only the most used two: MINUIT $]^{3}$ and its newer version NEWMINUIT. NEWMINUIT is a conservative optimizer that converges more slowly, but offers more accurate result and more reliable uncertainty estimates. The MINUIT manual suggests a maximum number of around 15 free parameters during the fitting process. This limitation was respected during our work.

This process is facilitated by the Fermitool gtlike that was used with the aid of Fermipy's function fit. The output are the parameters values that maximized the likelihood and their uncertainties, the value of $\ell$, the increase in $\ell$, in respect to the previous version of the model and the Test Statistic $(T S)$ value resulting from the fit. The $T S$ is described in Section 2.2.1.2,

\subsubsection{Model selection}

The maximum likelihood method provides the best fit parameters and the corresponding confidence intervals. However it does not include a measurement of the quality of the fit. The only way forward is to compare different potential models and select the one with the best fit to the data. The rationale behind this model selection is an underlying assumption that a higher probability of obtaining the data from a model (which means a model with a higher $\mathcal{L}$ ) signifies that this model offers a better description of the underlying reality than an adversary model.

The quantity used to select between two potential models is the $T S$, defined as

\footnotetext{
${ }^{3}$ cds.cern.ch/record/2296388/files/minuit.pdf
} 


$$
T S=-2 \log \frac{\mathcal{L}_{\max , 0}}{\mathcal{L}_{\max , 1}}
$$

where $\mathcal{L}_{\text {max }, 0}$ is the maximum likelihood value for the 'null hypothesis', and $\mathcal{L}_{\text {max }, 1}$ is the maximum likelihood value for a new model that is being evaluated.

Suppose we are interested in evaluating the existence of a source that is not currently part of the model. We can evaluate this possibility using Equation 2.11. We just maximize the likelihood for a model without the additional source $\left(\mathcal{L}_{\max , 0}\right)$ and for the model with the additional source $\left(\mathcal{L}_{\max , 1}\right)$ and apply Equation 2.11. Thus, a larger TS indicates that the null hypothesis is incorrect, i.e., a new source is a better explanation. As a general

rule, $\sqrt{T S} \approx$ significance $(\sigma)$ of the preferred model against the alternative (Mattox et al. 1996). Hence, in the example, the $T S$ would be approximately the square of the detection significance of the source in standard deviations. This is precisely the meaning of the $T S$ values we report, for instance in Table 3.1, for a given source.

This can be used to search for additional sources in the RoI (see Section 2.3.3). We just need to input an spectral model to a putative source and calculate, in an iterative way, the TS for a model with this additional source included in every pixel of the RoI. Since Equation 2.11 is a monotonically increasing function of $\mathcal{L}_{\max , 1}$, maximizing $T S$ on a grid is equivalent to maximizing the likelihood on a grid. Thus, locations in the map with $T S$ above some threshold indicate positions where additional sources may be included.

\subsection{Defining models with the Fermitools}

The analysis of Fermi-LAT data starts with the data selection and preparation, a process explained in section 2.1. A qualitative exploration of this data will present spatial clustering of photons, which hints for the presence of sources. But a quantitative study requires fitting models to this data. This necessity is justified by Fermi-LAT's effective area being dependent on the photon energy and angle of incidence; by the way Fermi operates scanning the whole sky every 3 hours, which results in a single source going from unexposed to the instrument to being exposed by several different angles in a matter of tens of minutes; by the instrument large PSF; and by the nature of the gamma-ray sky backgrounds.

We have chosen the size of the RoI taking into account Fermi-LAT's large PSF, specially 
at lower energies. This requires that counts detected in a region around the source of interest must also be taken into account. And the sources lying in this region have to be properly modeled as well. The influence of these other sources will be attenuated as they are farther away from the source of interest, which limits the size of the RoI. The ideal size of the RoI is a trade off between a more accurate model and the amount of data to process (which leads to higher computational demand). The optimal size is a matter of experimentation and experience.

As a starting point for our modeling of Fermi-LAT data, we used the 4FGL catalog of Fermi sources created by the Fermi-LAT Collaboration. The catalog contains the positions and spectral models of more than 5.000 gamma-ray sources detected during the first 8 years of Fermi operation. The parameters of these spectral models are the variables that can be altered to improve the quality of the fitting of the model to the data. In this process new sources can also be added to the model. We also considered two diffuse components to the model: the Galactic diffuse emission model (galdiff) and the extragalactic isotropic diffuse emission model (isodiff). The fitting of the model to the data is performed with a maximum likelihood technique.

\subsubsection{Universal Model}

Here we describe the process for the creation of the Universal Model with counts with energies between $100 \mathrm{MeV}$ and $500 \mathrm{GeV}$ and its offspring: the three models with energies between $300 \mathrm{MeV}-3 \mathrm{GeV}, 3-10 \mathrm{GeV}$ and $10-500 \mathrm{GeV}$. The data selection for this model was described in section 2.1.1

The region was modeled based on the preliminary release of 4FGL (gll_psc_v20.fit), the updated model of interstellar gamma-ray emission, gll_iem_v07.fits, and standard isotropic spectral templates selected according to the event types and event class used in this work. In the model, we included the 4FGL sources inside a square larger than the size of the RoI (with $25^{\circ}$ side) to account for Fermi-LAT's PSF. We performed a binned likelihood analysis using Fermitools conda package version 1.2.1, Fermipy Python package version 0.17.4 and Pass 8 release 3 Version 2 response functions (Atwood et al., 2013). Data was binned to a pixel size of $0^{\circ} .08$ and into 8 logarithmically spaced bins per decade in energy (for a total of 30 energy bins).

Fermi-LAT does not measure the energy of the photons with infinite precision. Energy 
dispersion is how the finite energy resolution of Fermi-LAT is called. It has been characterized by the Fermi-LAT Collaboration and this information is part of the instrument response functions. Turning on energy dispersion correction reduces systematic uncertainties. In this analysis, energy dispersion was disabled for the isotropic diffuse component only, as recommended by the Fermi-LAT Collaboration, since this component was created with data that did not take energy dispersion into account.

We performed a joint likelihood analysis with three components accounting for the isotropic emission because event types PSF1, PSF2 and PSF3 have different isotropic spectral templates.

We began the analysis using Fermipy's optimize method. It uses an iterative strategy to perform an automatic optimization of the model by fitting every source in the RoI. According to the Fermipy's developer: ${ }^{4}$, it is generally a good practice to run this method once at the start of every analysis to ensure that all parameters are close to their global likelihood maxima.

The first step of the optimize tool is to simultaneously fit the normalization of the $N$ brightest sources in the RoI that together sum up to a fraction npred_frac of the total predicted counts in the model. The $N_{\text {Pred }}$ for every source is a quantity easily calculated by fermipy based on their spectral models. We used npred_frac $=0.93$ which, based on previous analysis with similar characteristics, corresponds to $N \lesssim 15$. The next step is to individually fit the normalizations of every other source of the RoI, skipping any one that have $N_{\text {Pred }}<$ npred_threshold (we used the default value of npred_threshold $=1$ ). The last step is to individually fit the shape (the index) and normalization of all sources with $T S>$ shape_ts_threshold, where $T S$ is determined from the first two steps of the ROI optimization and shape_ts_threshold $=25.0$ (the default value).

After that we started fitting the sources in the RoI, using Fermipy's fit tool which performs a likelihood fit of all free parameters of the model and updates the characteristics of the corresponding model components (TS, $N_{\text {Pred }}$, spectral parameters, etc.).

Since the GC region is very rich in sources (section 1.5.5.2) we used an iterative approach on the fitting of the RoI, always taking care to limit the number of free parameters to less than 15 to avoid numerical issues 5 with MINUIT and NEWMINUIT, optimizers used

\footnotetext{
${ }^{4}$ fermipy.readthedocs.io/en/latest/fitting.html

5 fermi.gsfc.nasa.gov/ssc/data/analysis/documentation/Cicerone/Cicerone_Likelihood/Fitting_Models.html
} 
for maximizing the likelihood. Following the recommended procedure, we started every new round of fittig using the MINUIT optimzer until convergence for a rough likelihood maximization and them NEWMINUIT, a conservative optimizer that converges more slowly, for a more accurate result.

Several iterations were performed in order to fit the brightest sources closest to 4FGL J1745.6-2859. In all iterations, the normalization of our source of interest was allowed to vary as well as the normalization of galdiff and isodiff. The spectral models of these sources are, respectively, a log-parabola (Equation 1.2), a power-law (Equation 1.3) and a custom model (as explained in Section 1.5.5.1). The normalization of selected sources were also iteratively freed based on their proximity to the center of the RoI and their brightness in gamma-rays, obtained by the $N_{\text {Pred }}$ in each energy interval. We performed several iterations to fit the desired sources. Only the best quality fits, with Status: 0 and Quality: 3, were considered. The "Status: 0" flag indicates that the fitting procedure converged. And the "Quality: 3" indicates that the optimizer obtained a full accurate covariance matrix.

Fits that did not converge or with lower quality were disregarded. In this case we would go back to the previous step of the fitting procedure and continue from there with a smaller quantity of free sources.

The next step was to use the Fermipy function find_sources. This is an iterative source-finding algorithm that uses peak detection on a TS map to find new source candidates. In section 2.3.3 we delineate better what a TS map is. But, roughly, it indicates positions in the RoI where there is a high probability of existing a source not included in the model or a modeled source whose flux is 'under-predicted', which is not the case in this step of our analysis since we covered that in the previous fitting rounds. We used $T S>25$ - which translates to a significance $\gtrsim 5 \sigma$ of the existence of a new source in some region of the RoI - as a threshold to include new sources. After several rounds of this procedure, 29 sources were found. A power-law with index 2 was used as their initial spectral model. This spectral model was selected because it is typically adequate for relatively faint sources, and in fact the majority of sources in 4FGL are modeled with a power-law spectrum. Finding and characterizing new sources is not a goal of this work. The aim of this source-finding step was to improve the quality of the model. Some of the newly found sources are likely spurious detection due to unmodeled background emission. After they 
were found, some of them were refitted based on their distance to the central source, their brightness, or their proximity to regions with poor modeling (the evaluation of the fitting quality is detailed in section 2.3.3. The new sources are listed in Appendix C,

After every round of fitting we evaluated the quality of our fitting procedures using both:

- Residuals maps: built by subtracting modeled counts from the real data and searching for regions with significant residuals.

- TS maps: searching for the presence of an additional source component in each bin of the RoI.

In section 2.3 .3 we detail how these maps were created and used to evaluate the quality of our fitting.

The normalization of sources close to excesses in those maps was allowed to vary, together with the normalization of 4FGL J1745.6-2859 and of the diffuse components, in a new round of fitting.

The last step was fitting the index of our source. The approach was the same as described above. We performed different iterations of fitting, always with the normalization and the index of 4FGL J1745.6-2859 as free parameters together with other free parameters that usually included the normalization and the index of nearby bright sources and the galdiff and isodiff diffuse models. Once again, only iterations with the best fit quality were considered. By "index" of 4FGL J1745.6-2859 we mean the $\alpha$ and $\beta$ parameters of its Log-Parabola spectral model (Equation 1.2). The parameter $E_{0}$ was not freed during the fitting process.

These steps, after 86 rounds of fitting, led to the creation of the Universal Model with photons between $100 \mathrm{MeV}$ and $500 \mathrm{GeV}$. Then, it was used as a initial model for the analysis in three energy bands (300 MeV-3 GeV, 3-10 GeV and 10-500 GeV). In each energy band, the only sources that were allowed to vary in a single new round of fitting were 4FGL J1745.6-2859 (normalization and index) and the galdiff and isodiff diffuse models (normalization only). We used this "minimal fitting" approach with the objective of keeping a similar model in the three energy bands. By not fitting other sources of the RoI, we made sure that their spectral models were continuous between the three highest energy bands. In Appendix D, for the sake of clarity, we show examples of discontinuous 
models that we obtained in analyses in which the "minimal fitting" procedure was not used (they were not used in our work).

It is important to notice that we also created unique models, from scratch, to each of this three energy bands (300 MeV-3 GeV, 3-10 GeV and 10-500 GeV) and performed the fitting procedure reported in this section and the analysis detailed in section 2.4. The results we got with these unique models were compatible within $1 \sigma$ with the ones reported in sections 3.1 and 3.2 . We detail these comparisons in Appendix E.

Some results already naturally arise from the fitting procedure: we got the photon and the energy flux of 4FGL J1745.6-2859 between $100 \mathrm{MeV}-500 \mathrm{GeV}$ (the Universal Model), $300 \mathrm{MeV}-3 \mathrm{GeV}, 3-10 \mathrm{GeV}$ and $10-500 \mathrm{GeV}$. They are reported in section 3.1.

In addition to that we will use the Universal Model to obtain 4FGL J1745.6-2859's gamma-ray luminosity, SED and LCs in the Fermi energy range. Also, we used the 300 MeV-3 GeV, 3-10 GeV and 10-500 GeV models to evaluate the source position as a function of the photon energy. To improve this location study, we used a model created from scratch with photons in the $60-300 \mathrm{MeV}$ energy range as well. We explain how this model was created in the next section.

\subsubsection{Custom model (60-300 MeV)}

Our initial desire was to create a gamma-ray sky model in the lower energy band using the Universal Model as a starting point, following the same process described in section 2.3.1. But this proved to be challenging. We couldn't get a good quality model with the minimal fitting process nor with several additional rounds of fitting: in both cases we ended up with models whose Residuals and $T S$ maps showed many regions with excesses above the acceptable levels (i.e., $T S<25$ and Residuals $<|5 \sigma|$ ). So we decided to use a specific model to the 60-300 MeV energy band.

We considered data inside a $30^{\circ} \times 30^{\circ}$ square centered on the point source $4 \mathrm{FGL}$ J1745.6-2859. This choice of size for the RoI permit low energy photons to be properly modeled given the large PSF. We used $0^{\circ} .1$ pixel size and 8 logarithmically spaced bins per decade in energy.

We modeled the region using the preliminary release of 4FGL (gll_psc_v17.fit), the updated model of interstellar gamma-ray emission (gll_iem_v07.fits), and standard isotropic spectral templates. We included in the model all 4FGL sources in a region with 
$35^{\circ}$ side to account for Fermi-LAT's PSF. We performed a binned likelihood analysis using Fermitools conda package version 1.2.1, Fermipy Python package version 0.17.4 and Pass 8 release 3 Version 2 response functions. Energy dispersion was disabled for the isotropic and Galactic diffuse components.

As we did for the Universal Model (section 2.3.1), we performed a joint likelihood analysis with the three components accounting for the isotropic emission.

Before starting the analysis we changed the Spectrum Type of 4FGL J1745.6-2859. It is cataloged as log-parabola in 4FGL but we adopted a power-law in this low energy model. This change is appropriate since in small energy ranges (like the ones we are using here) a log-parabola can be approximated by a power-law. We used results obtained in previous analysis using the Third Fermi LAT catalog (3FGL, Acero et al. 2015), in which the source's spectra was modeled as a power-law, as the starting values for the parameters that were later refitted with the new data. The main reason for the change was the ease to fit power-laws, which have one less parameter compared to log-parabolas.

After that, we followed the same process described in Section 2.3.1 to fit the model until the source finding process. In this step, we encountered 14 new sources with Fermipy's function find_sources in regions associated with $T S>25$. But in new rounds of fitting, several of them showed a reducing in their TS to values below 25. Since Fermi LAT's PSF is poorer at lower energies, we decided to exclude - one at a time - the new sources in this condition from the model to avoid them interfering in the results of our source of interest. We started excluding the farthest from the center of the RoI and refitted the model. The normalization of the sources closest to the excluded one were let free, together with the normalization of the Galactic diffuse emission model, of the isotropic spectral template and of 4FGL J1745.6-2859. We repeated this process until there were no new sources with $T S<25$ in the model. After excluding these sources, we ended up with 5 new sources. These new sources may be spurious detections due to inaccuracies in the models. They are listed in Appendix $\mathrm{F}$.

In total, 67 rounds of fitting were performed in the process of creating a gamma-ray sky model for the 60-300 MeV energy range.

We also tried the same analysis with different combinations of event types: PSF2 + PSF3 (which means the 50\% photons with better PSF in the data) and front (that consider only the events that converted in the Fermi-LAT's "front", a part of the instrument that 
provides counts with better spatial resolution, as explained in section 1.5.1. Although we were not as thorough in the fitting process with this different event type selection- since we were just assembling preliminary tests - as in the analysis presented here, the results we obtained with them are all compatible with the values reported here in $<2 \sigma$. More details can be found in Appendix G.

\subsubsection{Evaluating the quality of the fitting process}

A new round of fitting was only considered valid if the model's likelihood increased, otherwise, it was discarded. This is implemented in Fermitools and Fermipy. But a simple increase in the likelihood was not enough to assure us that the new model was better than the previous one. For instance, some source's flux can become under-predicted (underpredicting the gamma-ray flux detected in that region of the sky) and the likelihhod can increase notwithstanding. In the case of this example, we would identify a excess in the diagnostic plots. The opposite (i.e. an 'over-predicted' source) would show-up in a region with negative excess in the residual maps. The maps would, then, indicate which sources models were sub-optimal or which region of the RoI demanded further modeling.

The set of diagnostic plots used in this work includes residual maps, TS maps (both of them briefly described in section 2.3.1 and residual histograms. In this section we explain them in length, delineate how they are created and in which way they are used to evaluate the quality of the models.

In every round of fitting the Fermitools (that we employ with the aid of Fermipy) use a maximum likelihood estimation (section 2.2) to fit a model to the available data. To do this, the software varies the selected spectral parameters of a sample of sources chosen by the user. It generates a three-dimensional (3D) distribution of counts: with two dimensions of space and a third dimension that considers the energy of the counts.

To create the spacial distribution, the positions and shapes of the sources in the model are convoluted with the instrument response (energy-dependent) functions to spatially distribute the model counts. For the point sources, only their positions are used. In the case of the extended sources, either a symmetric two-dimensional (2D) Gaussian (with a cataloged width), a symmetric 2D disk (with a cataloged radius) or an arbitrary 2D shape (with morphology defined by a template) is used as the spatial model. In the case of isodiff, its spatial model is a constant value in every position of the sky. And galdiff has also an 

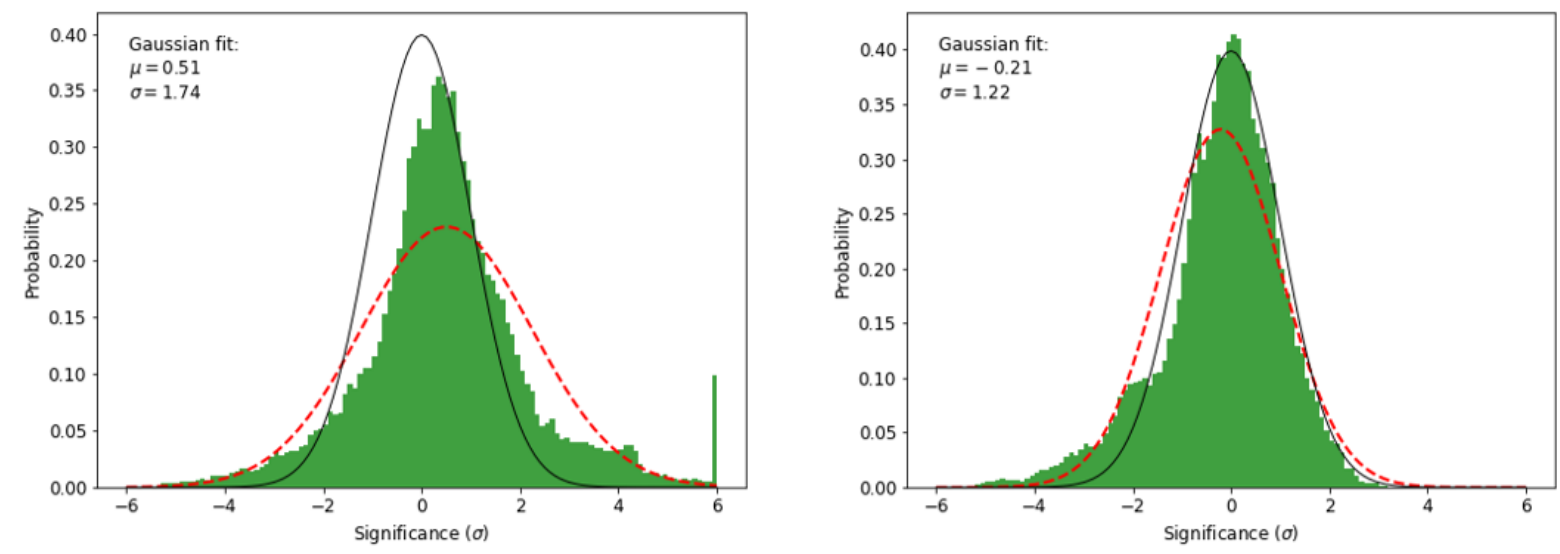

Figure 2.2: Examples with the residuals distribution for two steps in the fitting process of the $60-300$ $\mathrm{MeV}$ model. The panel on the left is from the beginning of the fitting process and shows a concentration of pixels with very high residuals, indicative of a poor modeling. The plot on the right is the same plot after the final step of fitting. The black line is a standard normal distribution and the red dashed line is the distributions for the best-fit Gaussian to the data.

arbitrary 2D model available through a .fits file.

This (after the process described in Section 2.3) generates the gamma-ray model. Comparing the model with the data (the detected photon counts) is straightforward. On simple way is to evaluate the residuals, calculated by subtracting the modeled counts from the real data.

One initial diagnostic tool is to evaluate the automatic-generated plots with the residuals distribution. In the case of a good model, one expects this to be a Normal distribution with mean $=0$ and $\sigma=1$ (a standard normal distribution). On the left-hand side of Figure 2.2 we show one of this histograms from an initial round of the fitting process of the low energy model (right after the use of the optimize tool). The one on the right-hand side is the final distribution of residuals for the same model. In Appendix $\mathrm{H}$ we show the resulting histograms obtained after fitting the three offspring of the Universal Model (i.e., $300 \mathrm{MeV}-3 \mathrm{GeV}, 3-10 \mathrm{GeV}$ and $10-500 \mathrm{GeV})$.

In both plots of Figure 2.2, the green blocks represent the probability (in \%) to find a pixel in the residual map (see Figure 2.3 as as example) with the significance indicated in the horizontal axis. This significance is calculated in units of standard deviation of the pixel value in respect to the mean value of all the pixels in the image. The black line is a standard normal distribution. The red dashed line is the distributions for the bestfit Gaussian to the data. The plot on the left, generated at the beginning of the fitting 
process, shows a concentration of pixels with very high residuals $(>6 \sigma)$, an indication that many counts are not attributed to modeled sources. The plot on the right was created after the last fitting: the concentration of pixels with high residuals is vanished and the best-fit Gaussian is closer to a standard normal distribution. The plots with the residuals distribution, however, were only used as a very preliminary analysis to the quality of the fit. Other diagnostic tools, described below, were always favored.

The results of our fitting steps discussed in Section 2.3 can be seen in Figures 2.3 and 2.4. These residual maps are useful to assess goodness-of-fit. The colors indicate the significance of the residuals in each pixel, calculated as the difference between the data and the model. Positive residuals indicate regions that are under-predicted whereas negative ones indicate over-predicted regions. Cyan crosses indicate the position of 4FGL sources and green crosses the position of new sources found during the analysis.

In the case of the Universal Model residual map (Figure 2.3) a few regions with negative residuals (in black) correlates with the extent of the Galactic plane. These regions are over-predicted, which means that the model predicts a higher gamma-ray flux than what was detected by Fermi-LAT. They are not associated with specific sources, which makes it harder to treat them. If that was the case, a simple round of fitting with the coincident source's parameter(s) freed could resolve that issue. In fact, their distribution along the Galactic plane suggests a strong association with the Galactic diffuse emission model (galdiff). As explained in section 1.5.5.1, the spatial distribution of this model follows observations in other wavelengths that try to map the interstellar gas distribution in the Galaxy. It is expected that the observational coverage varies for different regions. Our supposition is that the galdiff model is not successful in correcting for the lack of observational data in some regions. The same pattern shows up in the residual map of the $300 \mathrm{MeV}-3 \mathrm{GeV}$ (top right panel of Figure 2.4) but is not seem in the other panels that also originated form the Universal Model (bottom row of Figure 2.4), again, a strong suggestion that it may be originated from the galdiff since it is brighter in lower energies. Since the results we obtain through the Fermi data are based on the models, a consequence of this over-predicting would be a flux measurement higher than the reality for, at least, the $300 \mathrm{MeV}-3 \mathrm{GeV}$ energy band. But, as mentioned before (and detailed in the Appendix E the results we obtained in this energy range's model and with a different one with less regions of negative residuals excess were consistent with each other. Also, as will 


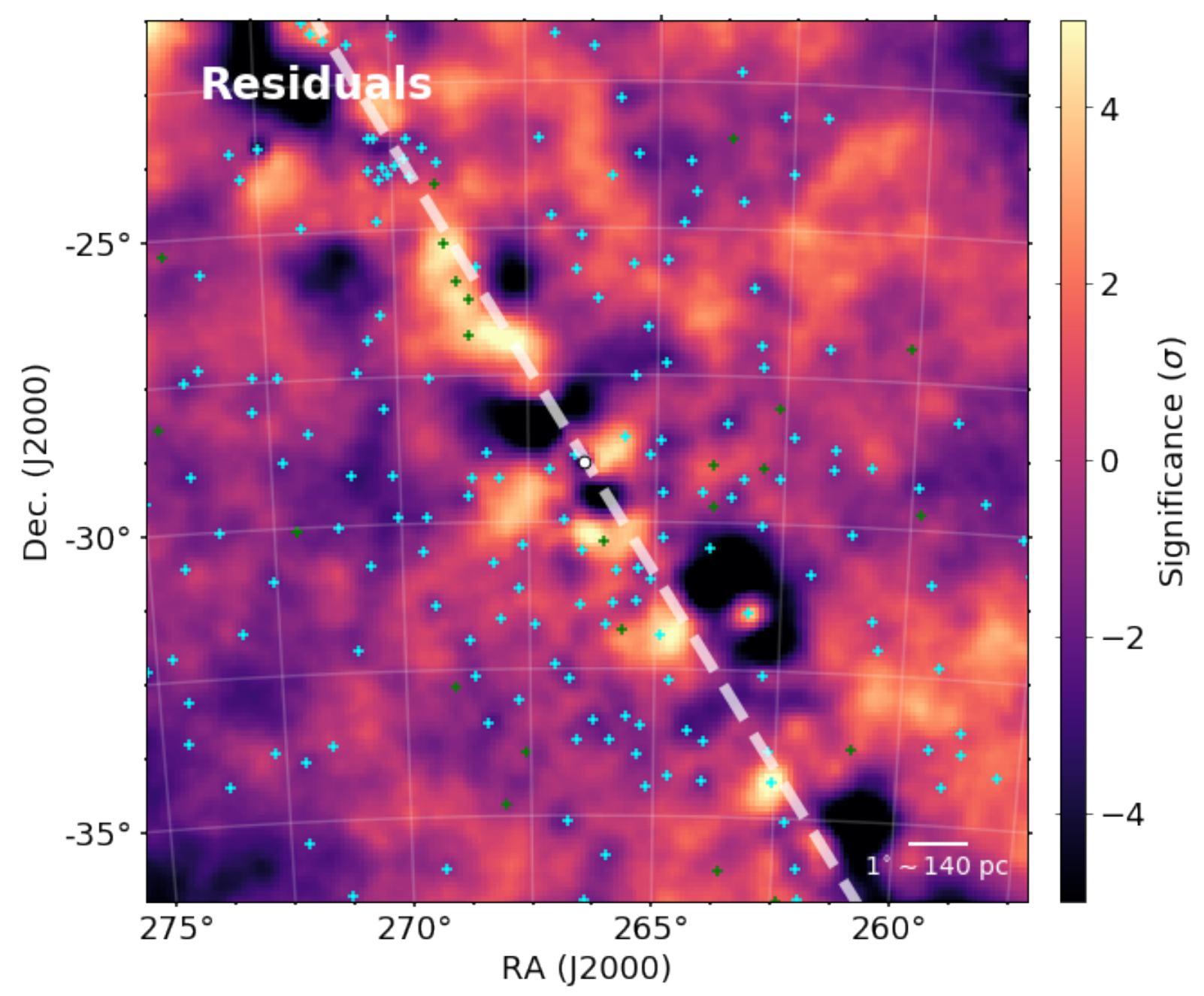

Figure 2.3: Residual map of the Universal Model created in the $100 \mathrm{MeV}-500 \mathrm{GeV}$ energy range. The colors show the significance of the residual. The point at the center of the image corresponds to the cataloged source position. 4FGL point sources are displayed as cyan crosses and new sources found during the analysis as green crosses. The white dashed line indicates the direction of the Galactic plane. 

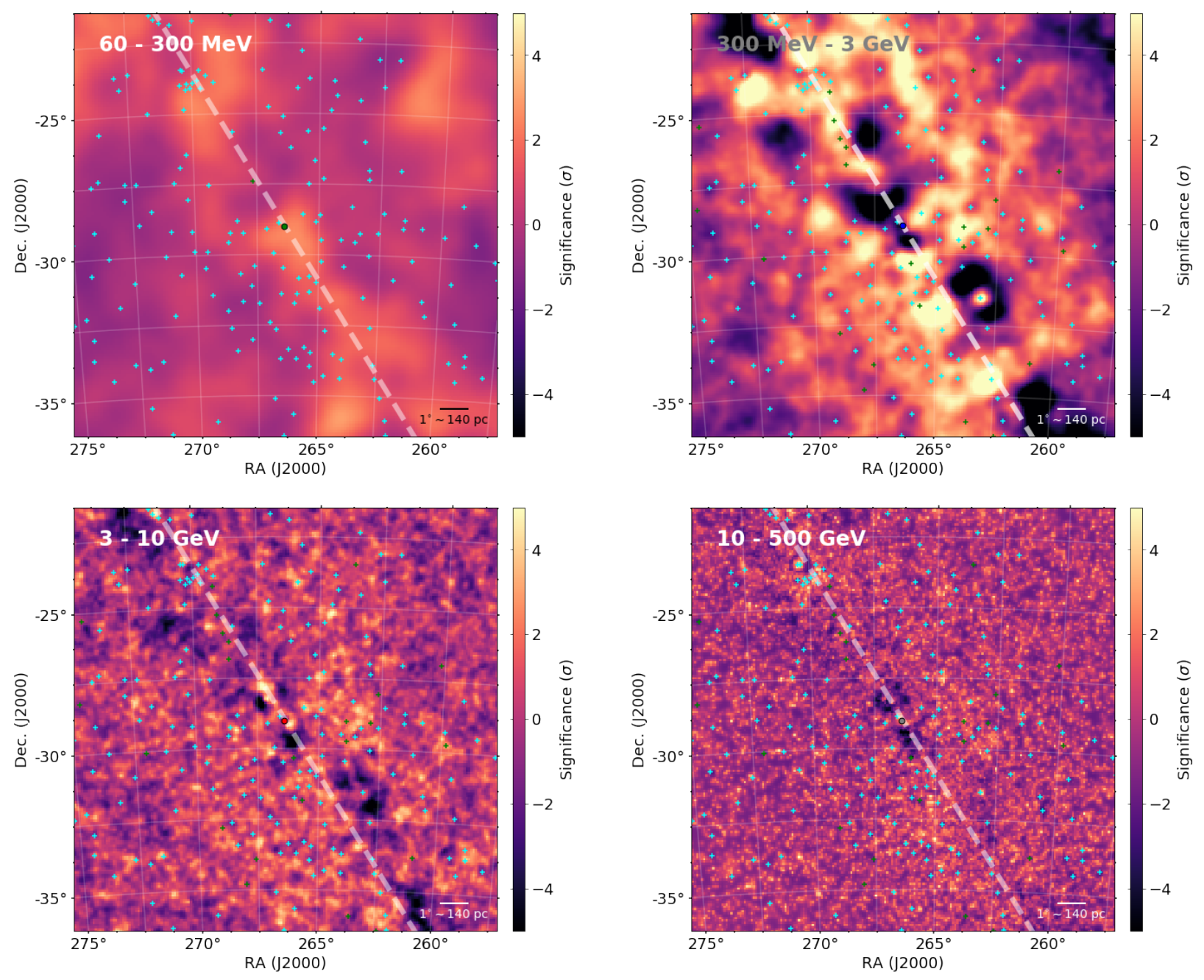

Figure 2.4: Residuals maps at the four different energy ranges. The colors show the significance of the residual. The point at the center of each panel corresponds to the source position obtained in each energy range. 4FGL point sources are displayed as cyan crosses and new sources found during the analysis as green crosses. The white dashed lines indicate the direction of the Galactic plane. 
be evident in Section 3.1, the spectral model for the source in the $300 \mathrm{MeV}-3 \mathrm{GeV}$ energy band is coincident with the one reported in 4FGL.

Another diagnostic plot that we generated to evaluate the quality of our fitting are the TS Maps. They are constructed with the Fermipy‘s tsmap tool. This tool moves a putative point source (with a power-law spectral model with a spectral index of -2) through the RoI and performs a maximum likelihood fit at each point. The $T S$ is a function of the maximum likelihood of a model with an additional source at a specified location (the putative source in this case) versus a model without it (section 2.2). The TS values computed for the presence of the putative source in each pixel devise the TS Maps. They are presented in Figures 2.5 and 2.6. The former regards the results for the Universal Model and the latter contemplate the results for the four tighter energy bands. In these maps, the colors indicates the $T S$ value at a given position. 4FGL J1745.6-2859 position (obtained with the method described in section 2.4 in each energy range is marked by a colored circle in the center of the images. The crosses follow the same pattern as in Figure 2.4.

The central point source itself is not visible in the maps since it is included in the models. For both the Universal Model (Figure 2.5) and for the 60-300 MeV energy band (Figure 2.6. upper left panel) there is no region with $T S \geq 25$ (i.e. no emission with significance $>5 \sigma)$. This shows that the presence of an additional source not already included in the models is unlikely. But in the maps of the high energy (>300 MeV) models this is not true. These models were created with minimal fitting based on the Universal Model (Section 2.3.1 which led to regions that could be better modeled if its sources were refitted and new sources were added to the models. Nevertheless, as we mentioned before, results we got for these energy models are compatible with the ones obtained through models created specifically to each energy band and that had no region with $T S>25$ (as presented in the Appendix E.

We also created different versions of the TS Maps. In this case, before using the Fermipy's tsmap tool, we removed 4FGL J1745.6-2859 from the model (and performed no additional fit). One expects, in this case, to observe $T S$ excess in the region around the position of the source. The goal here is twofold: first as a sanity check to confirm that the source is really there and to search for deviations from a circular region, characteristic of a point source.

The results of this process are shown in Figures 2.7 and 2.8 for the Universal Model 


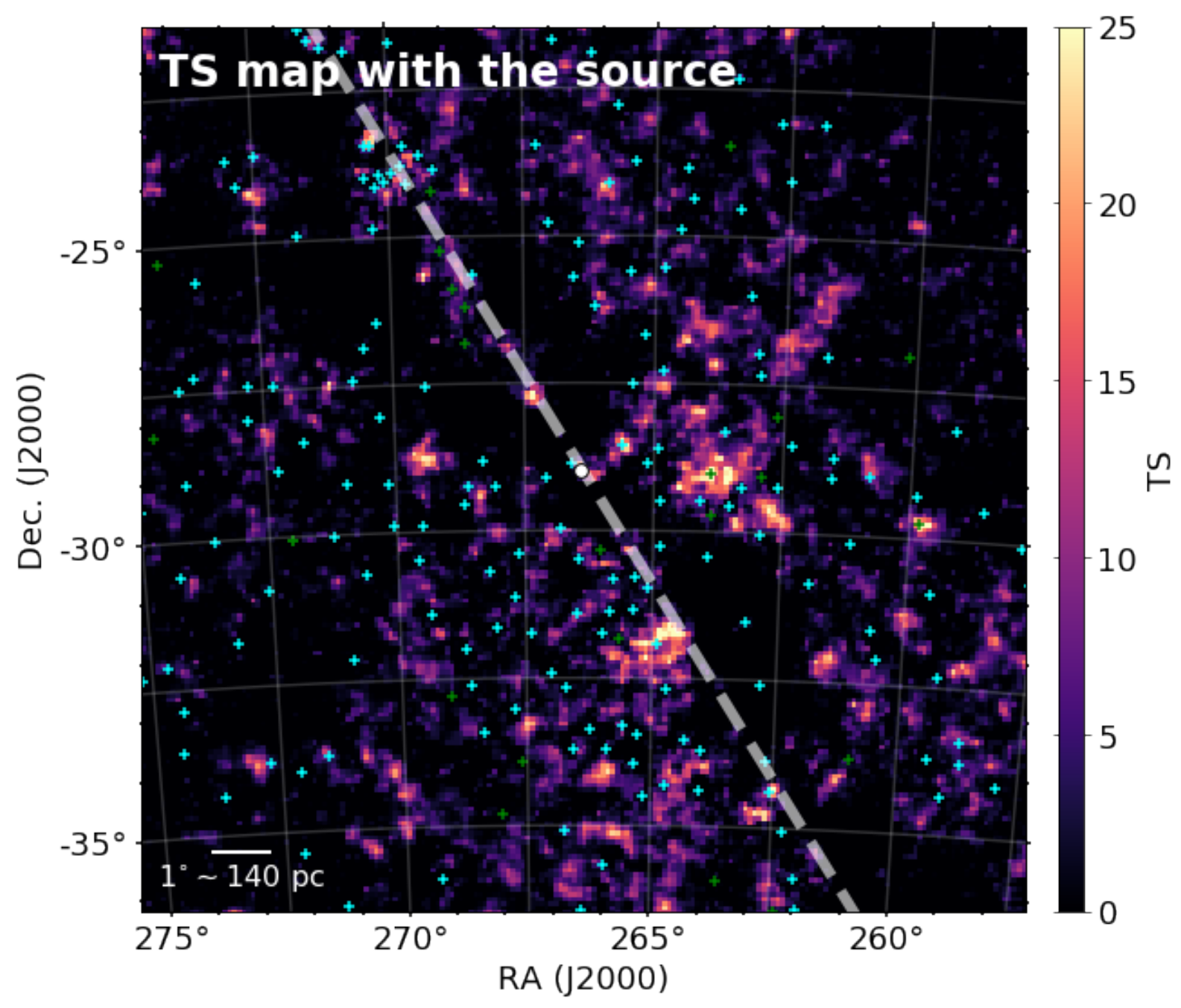

Figure 2.5: TS map of the Universal Model created in the $100 \mathrm{MeV}-500 \mathrm{GeV}$ energy range. The central point source is not prominent in this map since it is part of the model. The circle at the center of the image corresponds to the central point source cataloged position. 4FGL point sources are displayed as cyan crosses and new sources found during the analysis as green crosses. The white dashed lines indicate the direction of the Galactic plane. 

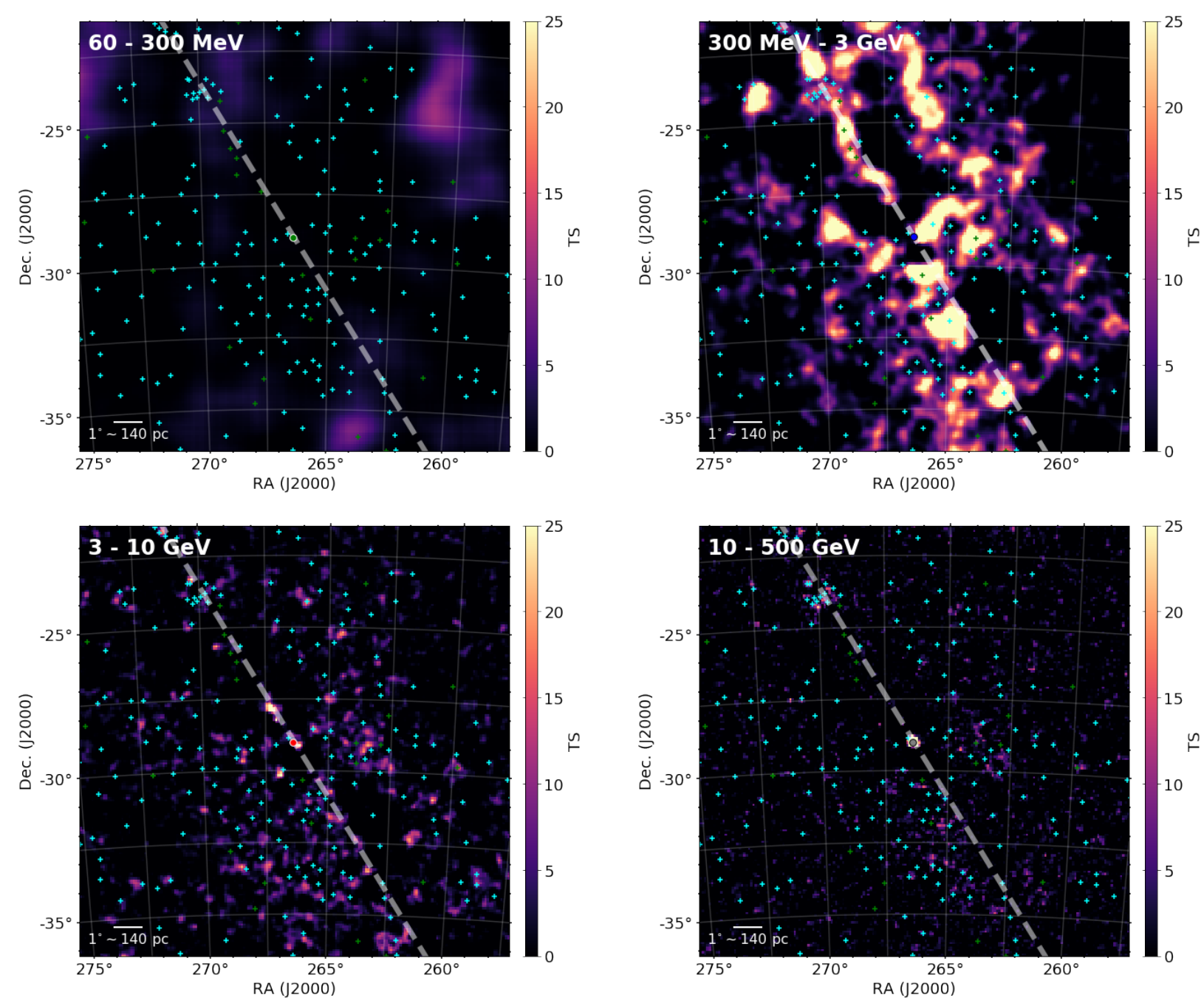

Figure 2.6: TS maps of the RoI at four different energy ranges. The circles at the center of the panels correspond to the central point source position obtained in each energy range. 4FGL point sources are displayed as cyan crosses and new sources found during the analysis as green crosses. The white dashed lines indicate the direction of the Galactic plane. 


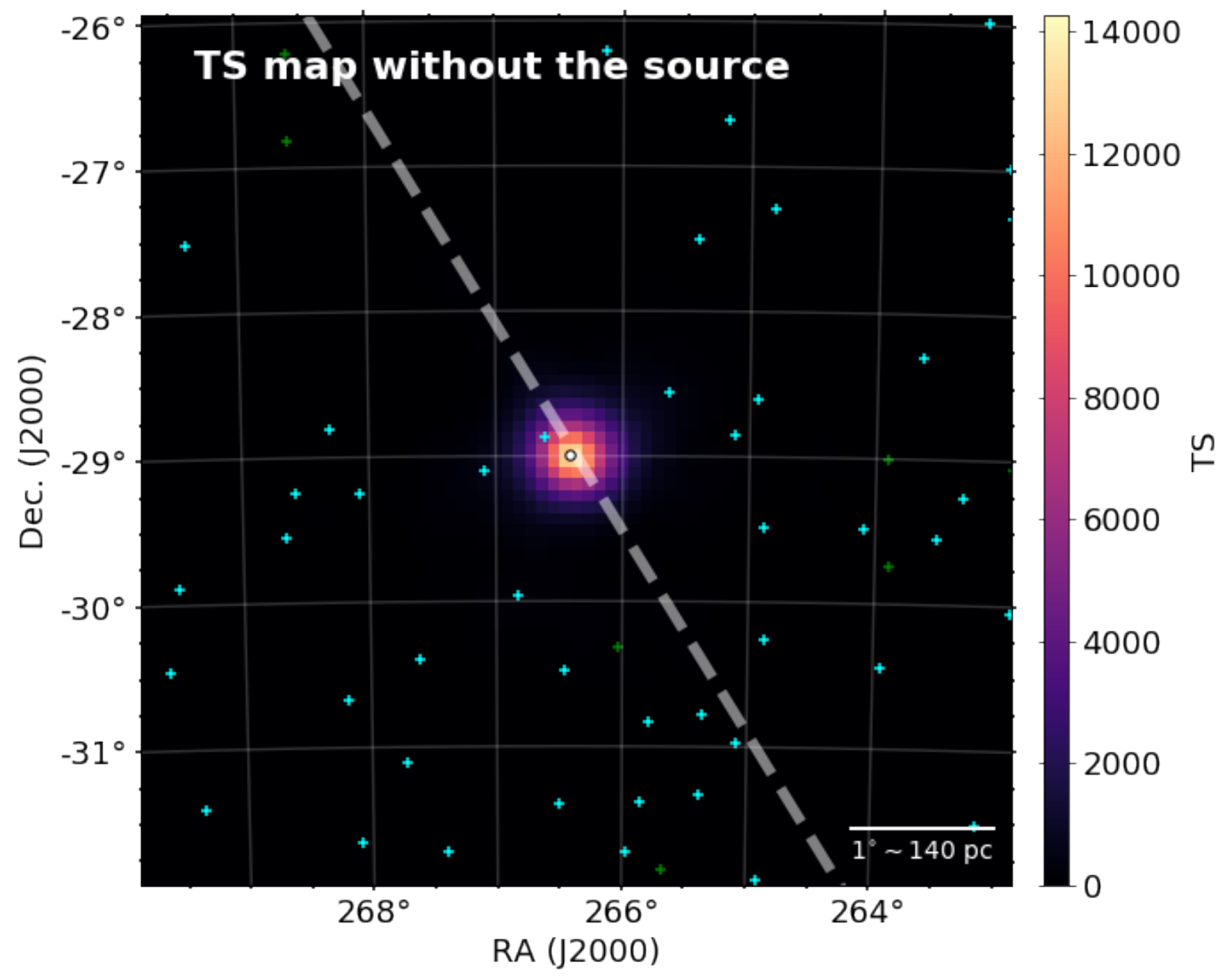

Figure 2.7: $T S$ map of of the inner $8^{\circ} \times 8^{\circ}$ of the RoI created for the Universal Model created in the $100 \mathrm{MeV}-500 \mathrm{GeV}$ energy range without 4FGL J1745.6-2859. The point at the center of the image corresponds to the source position in the catalog. The other 4FGL point sources are shown as cyan crosses and new sources found during the analysis are show as green crosses. The white dashed lines indicate the direction of the Galactic plane.

and the ones created with tighter energy bands, respectively. They show the TS maps of the inner $8^{\circ} \times 8^{\circ}$ of the RoI, evidencing that 4FGL J1745.6-2859 is detected with very high significance in the whole energy range used in this work. The colors and crosses have the same meaning as in the previous Figures. TS excesses are observed in both images and are always coincident with 4FGL J1745.6-2859's position. The size of the emitting region seems to decrease with energy but this is an outcome of the improvement of Fermi LAT's PSF with energy as mentioned in Section 1.5.3. Although we didn't perform a quantitative test, no clear deviations from a circle can be observed in these images. Further quantitative investigation on the point source hypothesis will be presented in section 2.4 .

The maps shown in this sections are for the final version of each model. But, they 

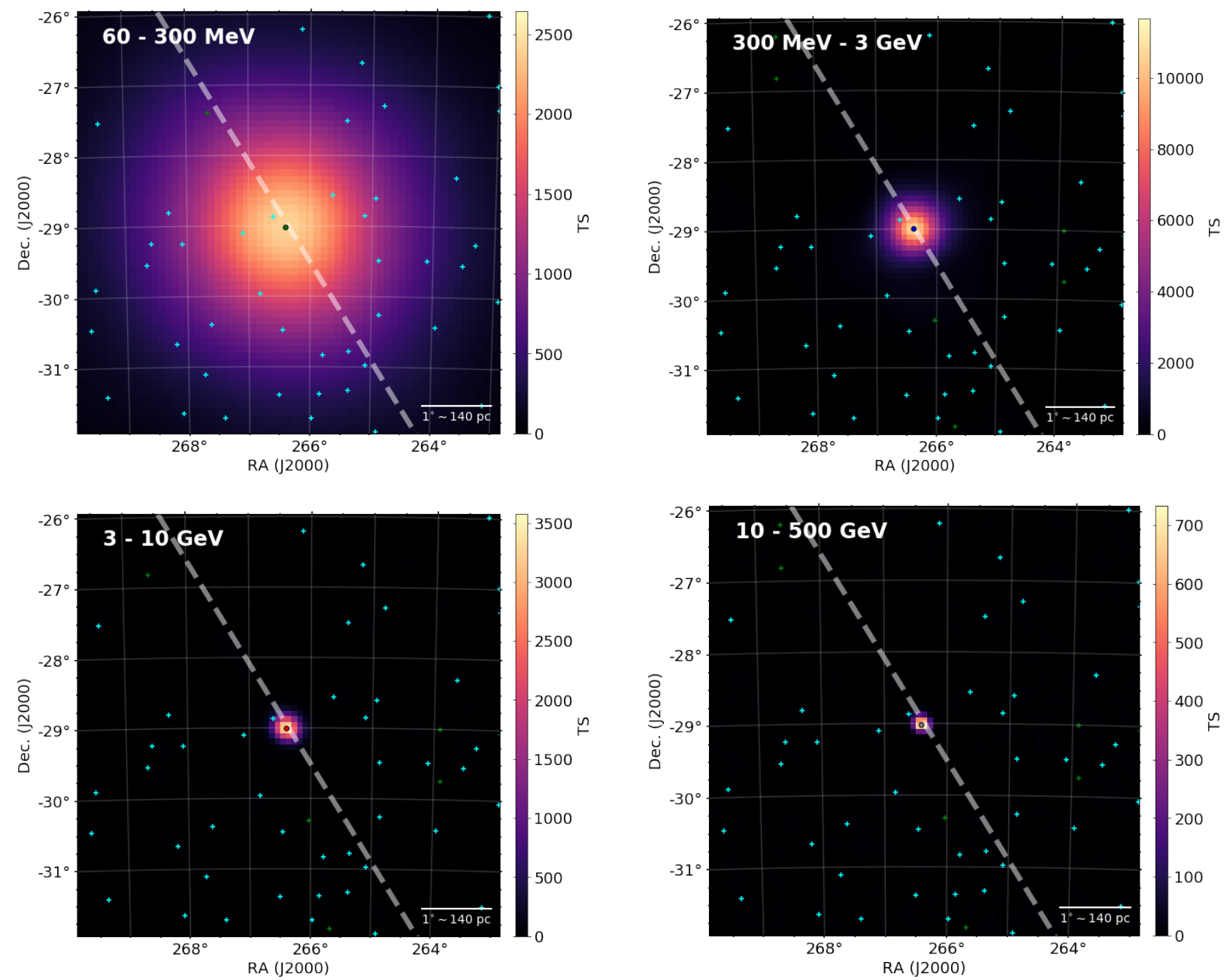

Figure 2.8: TS maps of the inner $8^{\circ} \times 8^{\circ}$ of the RoI evidencing the contribution of the central point source. They were constructed after excluding 4FGL J1745.6-2859 from the models, as explained in the text. The point at the center of each panel corresponds to the source position obtained in each energy range. The other 4FGL point sources are shown as cyan crosses and new sources found during the analysis are show as green crosses. The white dashed lines indicate the direction of the Galactic plane. 
were generated and evaluated at every fitting step of the analysis in order to identify sources and/or regions in the RoI that demanded further fitting. This was made by the identification of excesses in the images. In this case, at least the normalization of a source (or several sources) near or coincident with the excess was allowed to vary in a future round of fitting.

With the models created and evaluated, we can move on to the next steps of the analysis. In them, we assess the source position in the four energy bands we studied, its variability in time and its SED.

\subsection{Assessing the source position}

One of the goals of our work was to identify if 4FGL J1745.6-2859's position changes with photon energy, this was the main reason behind the division of the analysis into four different energy bands. To identify the source's position and calculate the likelihood of it being spatially extended, we used Fermipy's extension method. In addition to finding the location of the point source, it computes the likelihood of the source being extended with respect to it being pointlike and calculates the extension that maximizes the model likelihood.

\subsubsection{Identifying the source position with the Fermitools}

In this step of our work, we followed exactly the same process for the four tighter energy bands (60-300 MeV, $300 \mathrm{MeV}-3 \mathrm{GeV}, 3-10 \mathrm{GeV}$ and 10-500 GeV). We used Fermipy's extension method. This tool finds the location of the point source, computes the likelihood of the source being extended with respect to it being pointlike and, also, gives the best fit-model for the extension.

To localize the point-like source, the tool first scan the $T S$ map around the cataloged source position. Basically, it investigates maps very similar with the ones presented in Figures 2.7 and 2.8 with the difference that instead of using a power-law spectral model with a spectral index of -2 as the putative source, it uses the spectral model obtained in the last round of fitting. The spectral parameters of every source source in RoI are kept fixed in their current values. This creates a first estimate of its position. The size of 
the search region was a circle with radius $0^{\circ} .5$ centered in the original position of $4 \mathrm{FGL}$ J1745.6-2859. Them, in a second iteration, the tool refines the source localization by performing a scan of the likelihood surface in a box centered on the best-fit position found in the previous iteration. The size of this box circumscribe the $99 \%$ positional uncertainty contour obtained before. In this second round, the free spectral parameters of every source in the RoI are re-fit. If a peak is found in the likelihood surface and the positional fit succeeds the tool updates the position of the source in the model to the new best-fit position. In this step of the analysis, other than keeping every spectral parameter in the RoI fixed, we tried to let free several different combinations of the normalization and index of the following sources: 4FGL J1745.6-2859, galdiff, isodiff and the four brightest sources closer than $2^{\circ} .0$ from the central source. The fitting was not successful in most of theses analyses. Of the few of them that returned meaningful results, all of them were compatible in $1 \sigma$ with each other. We chose, than, to report (in Section 3.2 the one with the highest likelihood value which was the one with every parameter fixed. The model with the background sources fixed were the ones with the highest likelihood in the four energy bands studied.

With this method, we got the position (RA, Dec) of the point source in each of these energy bands and also the statistical uncertainty. In section 2.4 .2 we explain how we estimated the systematic uncertainty of this measurement.

Apart from computing its position in the four energy bands, we also tried to estimate if it was compatible with an extended source. This is also part of Fermipy's extension method. The method computes a likelihood ratio between a hypothesis of the source being point-like and a best-fit model for extension. To obtain the best-fit extension model, extension varies the source width (the $68 \%$ containment radius of the point source model) and finds the extension that maximizes the model likelihood. We used a 2D Gaussian as the spatial model for the source. We tried to free the same combinations of spectral parameters in the RoI as we did in the localization of the point-like source (described above). When the extension method works flawlessly, it returns the $T S$ of the extension (a measure of the likelihood between the extended versus the point-like hypothesis); the best fit and an UL for the extended source radius; and the location (RA and Dec) of the extended source center. We couldn't get good results in the vast majority of the tries in all energy bands. The most probable culprit for this is the strong source confusion. As we mentioned in 
Section 1.5.5.2, the GC is the most crowded region of the Fermi sky. The closest source to 4 FGL J1745.6-2859 is only $0^{\circ} .21$ away and there are 21 sources closer than $2^{\circ} .0$ from 4FGL J1745.6-2859, for instance. Because of that, we decided to report only the $95 \%$ confidence level UL of the extension (in Section 3.2). We took care to select the highest meaningful UL obtained in each energy band (presented in Figure 3.4).

\subsubsection{Systematic uncertainties}

To calculate the total error $\Delta_{t o t}$, on the location of the point source we followed the approach used by The Fermi-LAT Collaboration (2019) for the creation of the 4FGL Catalog:

$$
\Delta_{\text {tot }}^{2}=\left(f_{\text {rel }} \Delta_{\text {stat }}\right)^{2}+\Delta_{\text {abs }}^{2}
$$

where $f_{r e l}$ is the systematic factor, $\Delta_{\text {stat }}$ is the statistical error and $\Delta_{a b s}$ is Fermi-LAT's absolute precision (obtained by the Fermi-LAT Collaboration by comparing the location of the brightest cataloged sources with associations with their true positions in the sky obtained in other wavelengths).

For $\Delta_{a b s}$, we used the value of $0 .^{\circ} 0068$ (The Fermi-LAT Collaboration, 2019) in the two energy bands below $3 \mathrm{GeV}$ and $0 .^{\circ} 0075$ (Ajello et al., 2017) for higher energies bands (both values at the $95 \%$ confidence level). For $f_{\text {rel }}$ we used 1.1 for the three lowest energy bands and 1.2 for the $10-500 \mathrm{GeV}$ band. These values are estimated by the Fermi-LAT Collaboration with validation studies performed with the Vela pulsar and stacked highlatitude AGNs which are less subject to contamination from the diffuse Galactic emission. Its value is of $\sim 1.05$ in the $100 \mathrm{MeV}-10 \mathrm{GeV}$ and increases for lower and higher energies, as shown in Figure 1.16. We decided to use more conservative values since the source we are studying lies in the most densely packed region of the gamma-ray sky. The $\Delta_{\text {stat }}$ were the $68 \%$ positional uncertainty obtained during the analysis.

The uncertainties we report in Table 3.1 and Figures 3.2 and 3.3 correspond to the value of $\Delta_{\text {tot }}$ computed above. 


\subsection{Variability of the $G C$}

Fermi covers the whole sky every three hours since August 2008 therefore its data are specially suitable to investigate temporal behavior of a gamma-ray source. In this section we explain how, using Fermipy's method lightcurve, we created LCs for 4FGL J1745.6-2859. We used the Universal Model as a baseline. In a nutshell, this process is very similar to the fitting we used to create the Universal Model. But, now, we also separate the data in time bins.

Additionally in this section we present the methods we used to search for periodicity and variability in the LCs.

\subsubsection{Creating a light curve}

We start with the Universal Model and use the method lightcurve from Fermipy. This method fits a source, just like we described in Section 2.3, but now in a sequence of time bins. The tool returns several quantities in each time bin: flux, spectral parameters, $T S$, among others.

Since the creation of LCs is a very time consuming process, we used the SLAC National Accelerator Laboratory computer cluster, operated by Stanford University (as a member of the Fermi-LAT Collaboration, we have access to this resource). Even using the cluster, the creation of the LC takes a long period of time. The amount of data generated during the creation of a LC is huge: it is about the same volume of data created in the fitting process (of a few tens of GB) multiplied by the number of time bins. Because of storage limitation in the cluster, we had to split the LC in several smaller jobs. The creation of the $\sim 11.3$ years LC with 45 days bins (91 bins in total) took almost a month (in real time) to be generated.

It is possible to free other sources of the RoI so their spectral parameters can also vary during the fitting. It is important to avoid the contamination from other nearby sources variability in the LC of the source of interest. In order to save time, we investigated every source closer than $5^{\circ}$ from the center of the RoI in the 4FGL (total: 105 sources). The catalog offers a measurement of the probability of each source being steady (variability_index). We started by selecting all the sources with a value of variability_index consistent with a variable source (the sample reduced to 85 sour- 
ces). Then, we eliminated the sources with average flux smaller than $10 \%$ of $4 \mathrm{FGL}$ J1745.6-2859's average flux. We finished with only 4 sources that are: a) close to 4FGL J1745.6-2859; b) variable; and c) with flux above 10\% of 4FGL J1745.6-2859's. All of this sources are identified in the 4FGL as either a pulsar, a pulsar wind nebula or a globular cluster (whose gamma-ray emission is associate with mili-second pulsar; de Menezes et al. 2019). These sources are not expected to be variable in the time ranges we are working with. The diffuse sources (galdiff and isodiff) are also not variable. For this reason, we decided to let free only the spectral parameters of our source of interest during the creation of the LCs.

First, we created a LC with 45 days bins. Then, since we did not find any period of significant variability, we decided to create a LC with 15 days bins (which took more than 2 months of processing). Smaller time bins gives resolution for the LC. At the same time, the number of photon available for the fitting decreases, reducing the statistical significance of the detections.

The output of the lightcurve method is the source's photon and energy flux. It also gives the $T S$ of the source in each time bin. Using it, we can evaluate if the source was detected in specifics time bins and, if it was not, proceed accordingly reporting not the (energy or photon) flux but an UL. As a threshold for the detection significance in the time bins, we used $T S>16 \approx 4 \sigma$.

Additionally, we created three other LCs, using the three models created from the Universal Model in the $300 \mathrm{MeV}-3 \mathrm{GeV}, 3-10 \mathrm{GeV}$ and 10-500 GeV energy bands. Since the amount of counts in these models are significantly smaller than in the Universal Model ( $\sim 1 / 3$ of the counts), we had to work with larger time bins in order to obtain points with good significance. We decided for these sizes empirically, testing LCs with different times bins (> 45 days) and checking the source's TS. We ended with 90 days bins for the energy bands $300 \mathrm{MeV}-3 \mathrm{GeV}$ and 3-10 GeV, and 180 days bins for the remaining band.

We did not create LCs for the lowest energy band used in this work (60-300 MeV). The main reason is that the tighter energy bands LCs were created with the goal of searching for "echoes" in the gamma-ray flux. As will be clear in Figures 3.2 and 3.3 , the centroids of the emission in the 3 highest energy bands are displaced from the position of Sgr A* in reverse order of energy. We were interested in searching for some signal of variability in the inner centroid being reproduced in the outer centroids after the light crossing time 
between these regions. Figure 3.2 shows that this analysis was not possible for the 60-300 $\mathrm{MeV}$ energy band because its position has larger uncertainty and it is spatially consistent with the emission in the 3 higher energy bands.

With the LCs created, we proceeded looking for periods of variability of the source. We describe how we performed this search in Section 2.5.3.

\subsubsection{Systematic uncertainties}

In this section we estimate the contribution of systematic uncertainties in the LC. They arise from the (incompletely corrected-for) variation of the acceptance as a function of time, which impacts the flux measurements. This is governed by the systematic uncertainty on the effective area. This is between $3 \%$ and $\sim 20 \%$ (Figure 1.15), depending on the analysis conditions.

In order to refine the estimate of the systematic uncertainty, we can quantify the apparent flux variation of bright and steady sources such as pulsars. This method was developed by Dr. Benoit Lott (Centre d'Études Nucléaires de Bordeaux Gradignan, Université de Bordeaux) to the Fermi-LAT Collaboration.

The standard deviation of the apparent flux distribution is the quadratic sum of the statistical and systematic uncertainties. So we can simply subtract the statistical uncertainty from the ones obtained through the lightcurve method to get to the systematic uncertainty.

Dr. Lott made the estimation in 2008 using the first $\sim 2.5$ months of data from the Vela pulsar, which is a very bright gamma-ray source. The method starts with the creation of a LC for Vela with the flux normalized to 1 . Then, they find the mean value of the distribution of the uncertainties in the LC (i.e., the mean value of the error bar in the LC). They got $\sigma_{\text {mean }}=0.13$. Finally, they make the distribution of relative dispersion:

$$
\text { Rel. dispersion }=\frac{F_{i}-F_{\text {mean }}}{\sigma_{i}}
$$

The expected distribution for the relative dispersion, supposing that the error bars are purely statistical, is a Gaussian distribution with unity width. The standard deviation of this distribution was found to be 1.23 . Then, they simply estimate the systematic variation as: $0.13 \times \sqrt{1.23^{2}-1^{2}}=0.093$, which translates to $9.3 \%$ systematic uncertainty. Other 
members of the Fermi-LAT Collaboration, using different methods, arrived at the same value.

We follow Dr. Lott's method but using a different pulsar. We chose to use the 4FGL J1747.2-2957 source. This is identified as a pulsar (PSR J1747-2958) 5kpc away from Earth (Hales et al., 2009) that lies only $1^{\circ} .03$ from the center of the RoI. It is important to select a source close (in the plane of the sky) to the center of the RoI to assure that it is also subject to the same confusing environment as 4FGL J1745.6-2859. Also, since it is a source in the RoI, we could use exactly the same analysis used in the Universal Model, which made the comparison between the LCs possible. We started by simply running a LC focused in this source as we had done to 4FGL J1745.6-2859 (in Section 2.5.1). In Figure 2.9 we show the 15 days bins energy flux LC with the average flux normalized to 1.

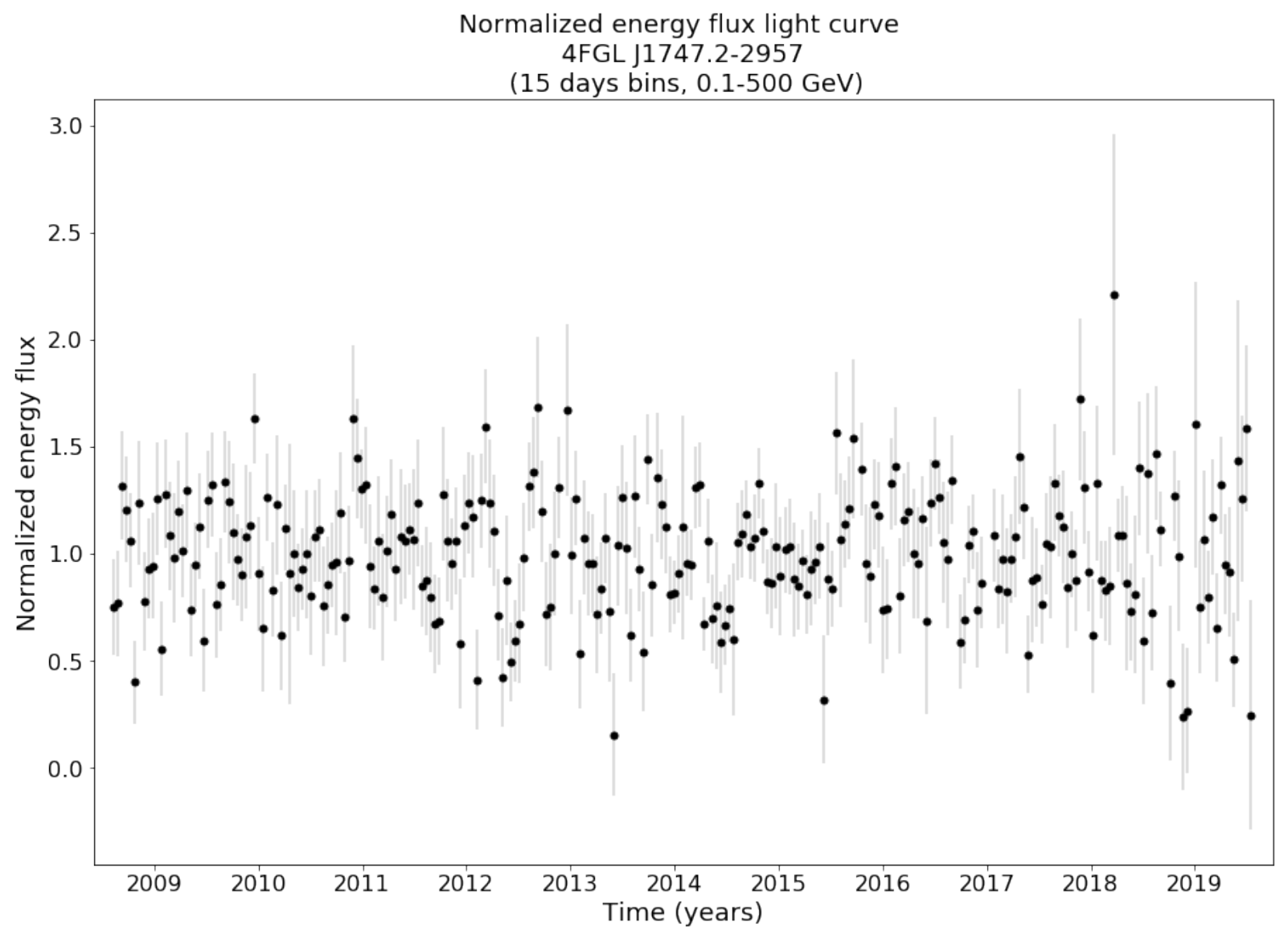

Figure 2.9: 15 days average normalized energy flux LC of 4FGL J1747.2-2957 (pulsar PSR J1747-2958). This is a presumably steady sources and its flux variability is used to estimate the systematic uncertainty in the data. This LC was created following the exact same data selection and methods used to create 4FGL J1745.6-2859 LC.

Then, we prepared the LC's uncertainties distribution, shown in Figure 2.10. We are 
interested in the average value of this distribution: 0.258 .

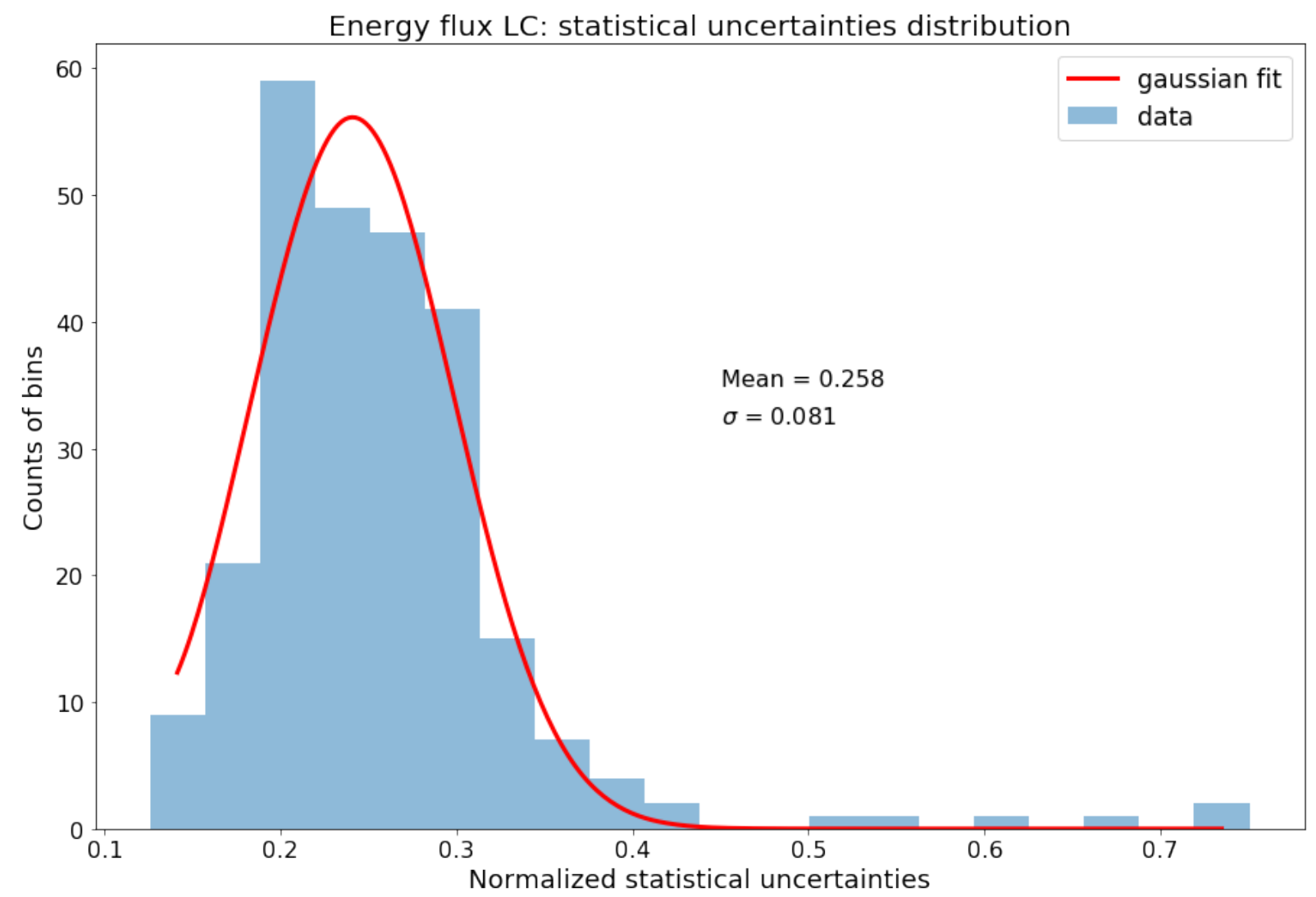

Figure 2.10: Histogram of the statistical uncertainties of the 15 days average normalized energy flux LC of 4FGL J1747.2-2957 (Figure 2.9). The dotted red line is a normal distribution with the same mean and standard deviation of the statistical uncertainties (the values are shown in the Figure).

The next step was to prepare the distribution of the relative dispersion. The value of the relative dispersion was calculated as in Equation 2.13. In Figure 2.11 we show the distribution of these values. The standard deviation of this distribution is 1.109. This leads to a systematic uncertainty of $0.258 \times \sqrt{1.109^{2}-1^{2}}=0.124$, or $12.4 \%$.

We followed this same procedure with the photon flux LC of 4FGL J1747.2-2957 and obtained a systematic uncertainty of $9.8 \%$ (the plots are available in Appendix I). These results indicate that the statistical uncertainty dominates the error bars in the LCs.

We also evaluated, using the same process described above, the systematic uncertainties of the other LCs we created, except for the 10-500 GeV energy band. In this particular case, we could not apply this method because the pulsar we used as a "standard candle" for the analysis (4FGL J1747.2-2957) is very weak in this energy band and hence it was not detected in the LC (the source has $T S \lesssim 5$ in every time bin of its 180 days LC). For this energy band we estimate the systematic uncertainty to be $<20 \%$. This is based on 


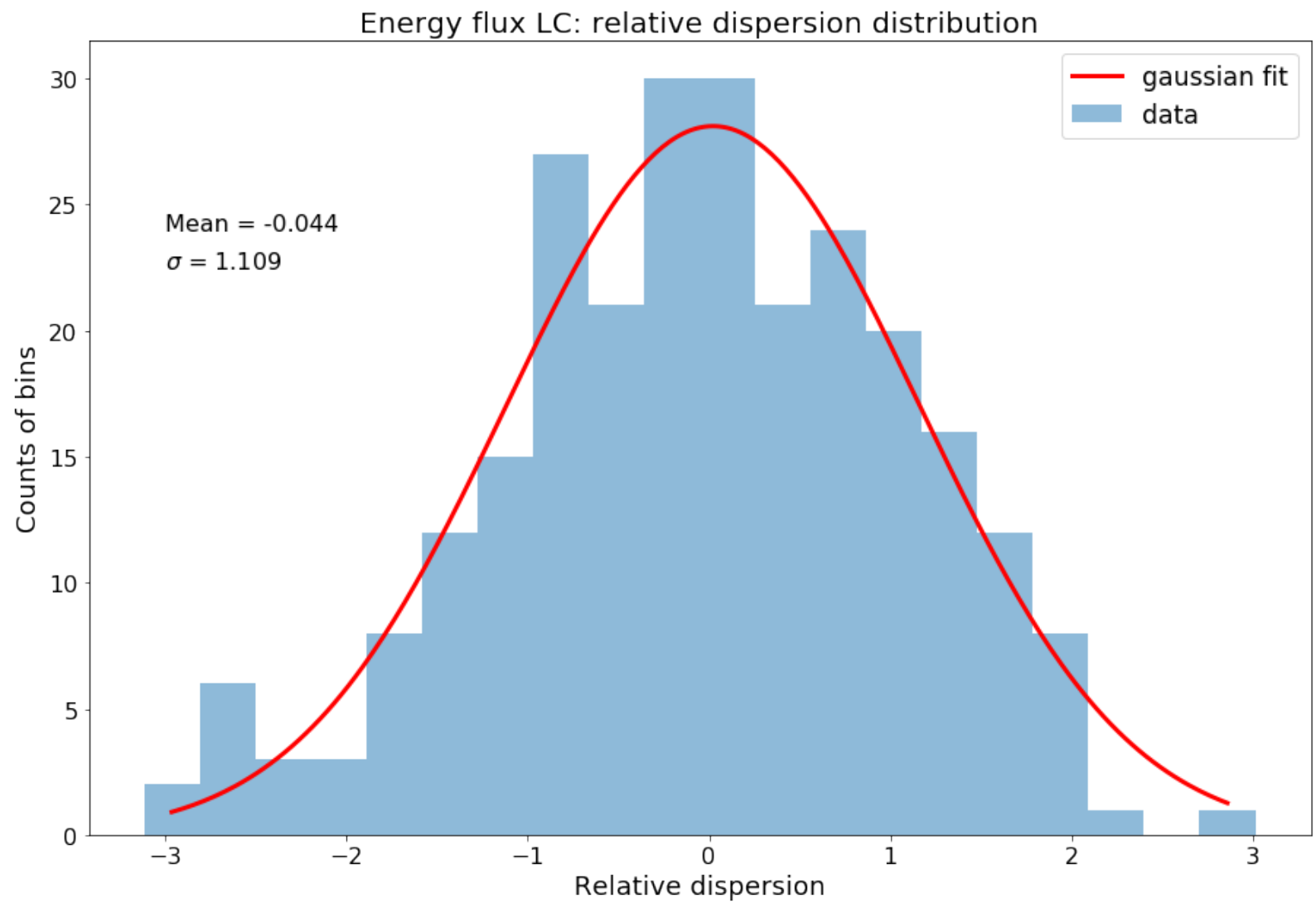

Figure 2.11: Histogram of the relative dispersion distribution of the 15 days average normalized energy flux LC of 4FGL J1747.2-2957 (Figure 2.9) calculated following Equation 2.13. The mean and standard deviation of this distribution are also shown in the Figure.

two arguments. First, the plot in Figure 1.15 indicates that $20 \%$ is an upper limit for this uncertainty. Also, the estimated systematic uncertainties of the two other energy bands are much smaller than $<20 \%$, and Fermi-LAT's effective area uncertainty decreases with the size of the time bin (180 days in this case, the largest used in this work). The values we obtained for all LCs reported in this work are listed in Table 2.1. In Appendix J we show the plots equivalent to Figures 2.9, 2.10 and 2.11 for the other LCs. We also report the relevant statistics to obtain the systematic uncertainties in each case.

Table 2.1 - The estimated systematic uncertainties of the LCs used in this work.

\begin{tabular}{cccc}
\hline \hline $\begin{array}{c}\text { Energy band } \\
(\mathrm{GeV})\end{array}$ & $\begin{array}{c}\text { Bin size } \\
\text { (days) }\end{array}$ & \multicolumn{2}{c}{ Systematic uncertainty (\%) } \\
energy flux & photon flux \\
\hline $0.1-500$ & 15 & 12.4 & 9.8 \\
$0.1-500$ & 45 & 9.8 & 9.0 \\
$0.3-3$ & 90 & 6.0 & 6.4 \\
$3-10$ & 90 & 9.0 & 7.0 \\
$10-500$ & 180 & $<20$ & $<20$ \\
\hline
\end{tabular}




\subsubsection{Searching for variability in the LCs}

Following the methods previously outlined, we now have these photon and energy flux LCs for the GC:

- with 45 days bins created based on the Universal Model (energy range: $100 \mathrm{MeV}-500$ $\mathrm{GeV})$;

- with 15 days bins created based on the Universal Model;

- with 90 days bins in the $300 \mathrm{MeV}-3 \mathrm{GeV}$ energy band;

- with 90 days bins in the 3-10 GeV energy band; and

- with 180 days bins in the $10-500 \mathrm{GeV}$ energy band.

As examples of the results we obtained, we show the 15 days bins energy and photon flux LCs in the upper and bottom panels of Figure 2.12, respectively (the other LCs are shown in Section 3.3). They are used to demonstrate the methods we employed to search for variability in the LCs. The error bars correspond to the $68 \%$ confidence level uncertainty. For the time bins in which the $T S$ of the source is $<16$, we show the $95 \%$ confidence level UL. 


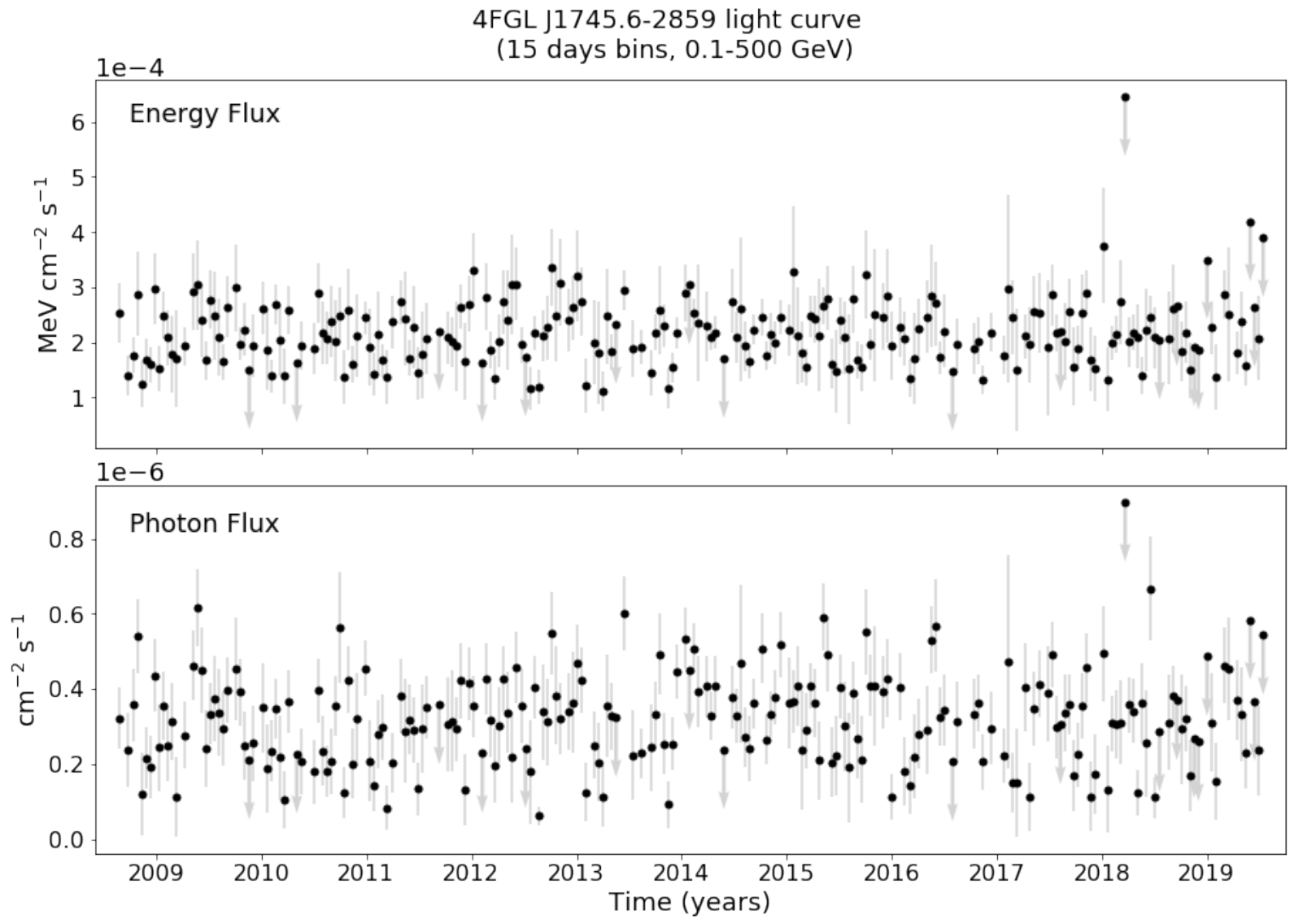

Figure 2.12: 15 days bins LCs of 4FGL J1745.6-2859 in the $100 \mathrm{MeV}-500 \mathrm{GeV}$ energy band. In the upper panel we show the energy flux and in the bottom panel we show the photon flux LC. The error bars correspond to the $68 \%$ confidence level uncertainty. For the time bins in which the $T S$ of the source is $<16$, we show the $95 \%$ confidence level UL.

A visual inspection of both panels of Figure 2.12 (and the others shown in Section 3.3) suggests no significant time variability. We tested this hypothesis using several different approaches that we described below in order of growing level of complexity. In the analysis below, we focus on the 15 days bins LCs created for the $100 \mathrm{MeV}-500 \mathrm{GeV}$ energy band. Since the 15 days bins LCs have much better time resolution than the 45 days bins, we disregard the later along this thesis. If the reader is interested in the 45 days bins LCs, they are addressed to Appendix $\mathrm{K}$. Some of the plots regarding the other LCs are shown in Section 3.3, when appropriate.

First, we calculated the mean flux during the whole period covered by the LCs $(\sim 11.3$ years) to compare with the flux in each bin. This is shown in Figure 3.6 at Section 3.3 . The flux observed in most of the time bins is compatible with the average flux, what is an indication that the source is in a quiescent state most of the time. This does not rule out the hypothesis of variability, of course. We decided to use the rolling average of the flux to 
look for bins that with fluxes significant higher (or lower) than the neighboring bins. We calculated the rolling average flux in groups of 5 bins consecutive bin ( $=75$ days in total). The rolling average values were plotted on the date corresponding to the central of the 5 bins in Figure 3.7 (Section 3.3).

These comparisons with the average fluxes are only preliminary examination of the data. They did not show any sign of variability, though. We moved on to more detailed and statistically supported methods.

We can check if the variability in the LCs arises from a random process by observing its probability density function. It can be estimated by fitting a model function to histograms of the observed flux (and its logarithm). We used a Gaussian distribution, representative of a normal random process. Also, there are evidence suggesting that the gamma-ray fluxes in blazars, intrinsically variable sources, are preferentially log-normally distributed (Sinha, A. et al., 2016; Shah et al., 2018). Log-normal flux variability is not unusual for accreting galactic sources (such as X-ray binaries, Uttley and McHardy 2001) and it has been linked to the underlying accretion process. We tested, therefore, whether the flux distributions are distributed normally or log-normally.

To inspect if 4FGL J1745.6-2859 flux variation in time is normally (representative of a random process) or log-normally (compatible with a the flux distribution of a flaring source) distributed we performed 3 different tests to quantify whether the data follow a Gaussian distribution. The results of theses tests are in Section 3.3 (Tables 3.2 and 3.3 ). The first is the Shapiro-Wilk test (Shapiro and Wilk, 1965), that quantifies how likely it is that the data was drawn from a Gaussian distribution. The second is the D'Agostino's $\mathrm{K}^{2}$ test (D'Agostino, 1971; D'Agostino and Pearson, 1973), a goodness-of-fit measure of departure from normality. This test is based on transformations of the sample kurtosis (that quantifies how much of the distribution is in the tails) and skewness (a quantification of how much a distribution is asymmetric). Finally, the Anderson-Darling test (Stephens, 1974) evaluates whether a data sample comes from one of among many known data samples (in our case, a Gaussian distribution). We used the open-source Python library SciPy (Virtanen et al. 2020) functions scipy.stats.shapiro, scipy.stats.normaltest, and scipy.stats.anderson to do the testings. To perform the tests, we assumed that our data followed a Gaussian distribution (or a log-normal distribution for the logarithm of the photon flux), which means that this was the null hypothesis $\left(H_{0}\right)$ we were trying to 
reject.

For the Shapiro-Wilk and the D'Agostino's $\mathrm{K}^{2}$ tests we used the p-value $(p)$ obtained to decide whether we reject $H_{0}$ or not. The p-value is interpreted as the probability of obtaining test results at least as extreme as the results actually observed, under the assumption that the null hypothesis is correct. We chose $5 \%(\alpha=0.05)$ as a threshold. If a test resulted in an $p>\alpha$ we would consider it a fail to reject $H_{0}$, hence the distribution was considered compatible with a Gaussian (or log-normal, when appropriate) distribution. Otherwise, we would consider the distribution not Gaussian (or not log-normal).

Each of these SciPy tests also returns a test statistic, a quantity that can be interpreted in the context of the test via comparing it to critical values from the distribution of the test statistic relevant to each test. For the Anderson-Darling test we compared the statistic with a list of critical values returned by the function, which allows a more thorough interpretation of the result. A test with statistic $>$ critical value results in a rejection of $H_{0}$.

Finally, we tested if the source flux displayed a global trend during the $\sim 11.3$ years observation. To do this, we simply fitted a linear function to the observed data following the Markov chain Monte Carlo (MCMC) method presented by Hogg et al. (2010) with the aid of the emcee Python implementation (Foreman-Mackey et al., 2013). During the fitting process, the error bars of our data were taken into account.

To assess if the LCs have a global trend in time, we are interested in the angular component of the linear fit, which gives the slope of the function and, hence, shows if the source's flux increased, decreased or kept constant in the observed period. Angular components close to (or compatible with) zero indicate that there is no measurable global trend in the data. In Section 3.3 we show the results of these fittings.

All these tests and analyses were performed for the 15 and 45 days LCs created in the $100 \mathrm{MeV}-500 \mathrm{GeV}$ energy range. For the other LCs (the ones with 90 days bins in the $300 \mathrm{MeV}-3 \mathrm{GeV}$ and in the 3-10 GeV energy bands and the one with 180 days bins in the 10-500 GeV energy band) we did not accomplish the Gaussian fit. The reason is the lack of statistics caused by the larger time bins and consequently small number of time bins. 


\subsection{Spectral Energy Distribution}

In this section we explain how to use Fermi data in order to create the SED of a source. Again, the process is very similar to the one used to create the Universal Model. But, now, we separate the data in energy bins channels. Each channel will give rise to one point in the SED. We used the Universal Model as the baseline. When the Universal Model was created (Section 2.3.1) we turned on the energy dispersion correction to reduce the systematic uncertainties in the measurements.

We used Fermipy's sed method. It performs independent fits for the flux normalization of a source in bins of energy. The source's original spectral model (the one obtained after fitting the Universal Model) is used as prior. The normalization in each bin is fit using a power-law spectral parametrization with an index that can be fixed or allowed to vary over the energy range according to the local slope of the original spectral model. We explored both possibilities.

We chose to split the energy band $(100 \mathrm{MeV}-500 \mathrm{GeV})$ in 8 bins per decade of energy, which accounts for 30 bins in the SED. Since Fermi-LAT's energy resolution varies with energy, it is important to choose an energy binning that is fine enough to capture this energy dependence and, at the same time, that is wide enough to have sufficient counts to get statistically significant results. Previous versions of this analysis indicate that using more than 8 bins per decade leads to many points with low significance. This empirical test is corroborated by the Fermi-LAT Collaboration's suggestion ${ }^{6}$ of using between 8 and 10 energy bins per decade.

During the SED creation, we can chose to also free other sources in the RoI. We tried different combination of free parameters, going from zero to a total of 5 free (normalization only) background sources. The background sources allowed to vary were selected among the brightest ones in the central $4^{\circ}$ of the RoI plus galdiff and isodiff.

We created a total of 7 SEDs with different selection of free parameters. To chose which one to report, we used the Akaike Information Criterion (AIC) Akaike, 1974). Given a collection of models (in our case, the resulting SEDs are models, as explained in Section 2.2 for a set of data (the observed counts), this method estimates the quality of each model, relative to each of the other models. Thus, AIC provides a means for model selection.

\footnotetext{
${ }^{6}$ fermi.gsfc.nasa.gov/ssc/data/analysis/documentation/Pass8_edisp_usage.html
} 
This allows us to select the best SED. The best-fit model according to AIC is the one that explains the greatest amount of variation using the fewest possible independent variables. AIC can be calculated as:

$$
A I C=2 K-2 \log (\mathcal{L})
$$

where $K$ is the is the number of independent variables in the model and $\log \mathcal{L}$ is its $\log$ likelihood. Models with lower AIC are considered better.

We calculated the AIC for the 7 models. The best model according to this criteria had the following free parameters: normalization and the index of 4FGL J1745.6-2859, the normalization of 3 point sources (4FGL J1747.2-2957, 4FGL J1742.5-2833 and 4FGL J1745.8-3028e) and the normalization of the diffuse components (galdiff and isodiff).

During the SED creation, the data is split into energy bins. The TS of the source of interest (see Section 2.2.1.2) is calculated for every bin (similarly as in the LCs creation). We decided to report the $95 \%$ confidence level UL for the energy bins in which the source was detected with $T S<5$ (which corresponds to $\lesssim 2.2 \sigma$ ). For the other energy bins, we report the energy flux $\left(E^{2} d N / d E\right.$, see Equations 1.1 to 1.3 and the $1 \sigma$ uncertainty.

The resulting SED is reported in Section 3.4 . 
Chapter 3

\section{Results}

In this section we present the results obtained with our work for the gamma-ray properties of the GC. We start with 4FGL J1745.6-2859's spectral models. Then we report its gamma-ray fluxes in four different energy bands. After that, we move on to the updated position of the source in the same bands. Finally, we describe the variability of the source and its SED.

\subsection{Spectral model}

In our work, we subdivided the analysis into four energy ranges. In Figure 3.1 we compare the results of these four models with the log-parabola spectral model adopted in the 4FGL Catalog for 4FGL J1745.6-2859. We can see there is a considerable discrepancy between the low energy custom model $(60-300 \mathrm{MeV})$ and the Universal Model. This is the result of the different modelling for the 60-300 MeV band, as discussed in Section 2 . Only part of this difference can be explained by the addition in the $60-300 \mathrm{MeV}$ model of a new source which is not included in 4FGL but listed as PS J1750.6-2723 in Appendix F, at a distance smaller than $2^{\circ}$ from 4FGL J1745.6-2859. This difference means that the gamma-ray flux for this energy band might be underestimated. It is important to notice that the GC is among the most complicated regions in the sky to study with Fermi LAT data: in addition to the high density of sources, the region is also engulfed by the Galactic diffuse emission. These issues become more severe in lower energies due to the large PSF.

The central source was detected in the four energy ranges used in the analysis with $T S$ varying from $\sim 300$ to $\sim 10000$, corresponding to detections with significance above 


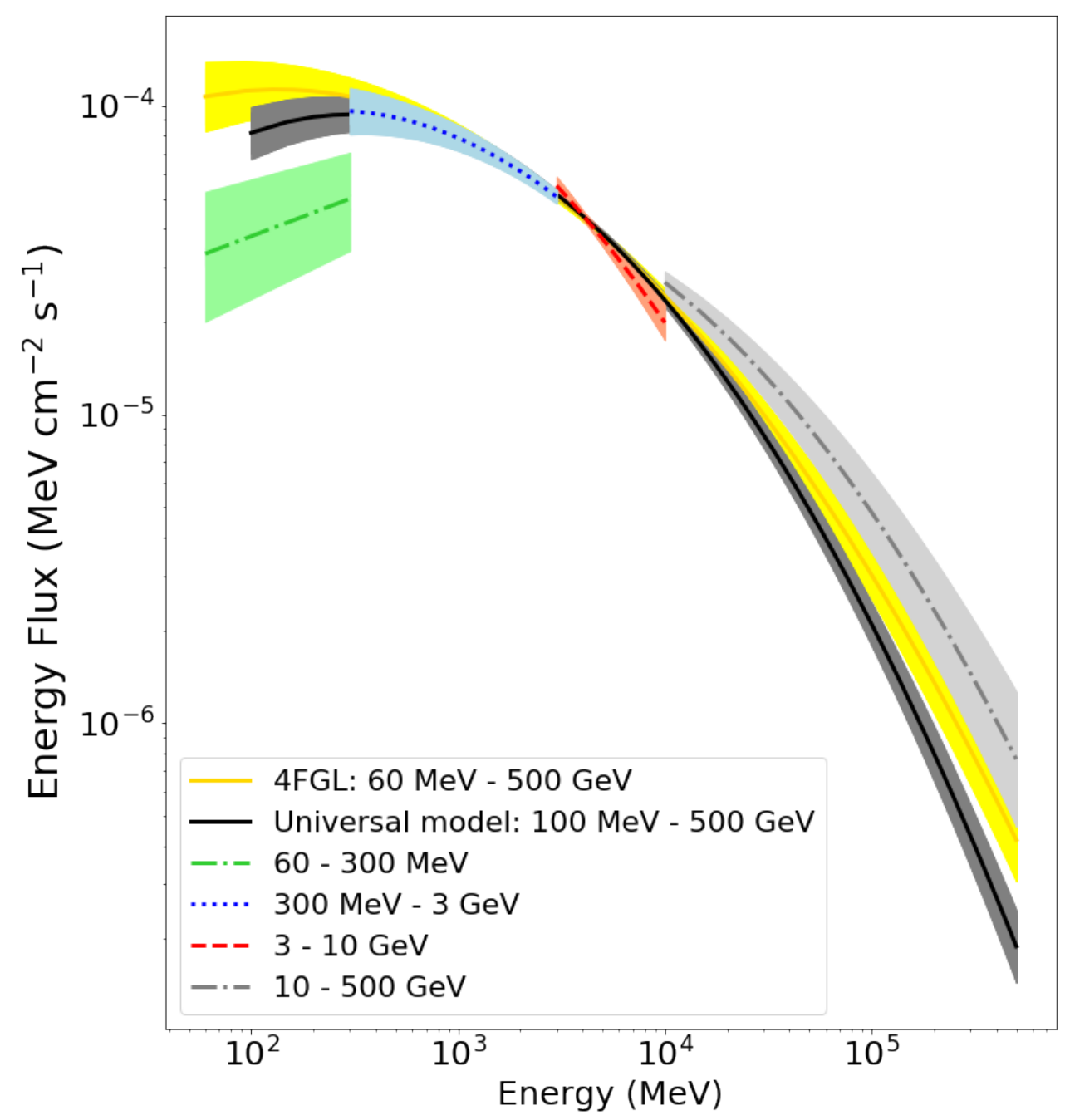

Figure 3.1: Comparison of the best-fit spectral models for 4FGL J1745.6-2859. The black line shows the Universal Model created in the $100 \mathrm{MeV}-500 \mathrm{GeV}$ energy range. It was later used as basis to create models in three different energy bands: $300 \mathrm{MeV}-3 \mathrm{GeV}$ (blue), 3-10 GeV (red) and 10-500 GeV (gray). They were created with log-parabolas templates. A power-law model was used in the 60-300 MeV energy range (green). The log-parabola spectral model used by the 4FGL Catalog for this source is also shown (yellow). 
background ranging from $\sim 17 \sigma$ to $\sim 100 \sigma$. Its photon and energy flux were also measured and the results are shown in Table 3.1. We also report the results of the Universal Model (100 MeV-500 GeV).

Table 3.1 - Results from likelihood modeling of the central point source. The last line presents the results for the universal model. The photon and energy flux uncertainties include only statistical errors.

\begin{tabular}{|c|c|c|c|c|c|c|c|}
\hline $\begin{array}{c}\text { Energy } \\
\text { range }(\mathrm{GeV})\end{array}$ & $\mathrm{TS}^{1}$ & $\begin{array}{l}\text { Photon flux } \\
\left(\mathrm{cm}^{-2} \mathrm{~s}^{-1}\right)\end{array}$ & $\begin{array}{c}\text { Energy flux } \\
\left(\mathrm{erg} \mathrm{cm}^{-2} \mathrm{~s}^{-1}\right)\end{array}$ & $\begin{array}{c}\text { Centroid }^{2} \\
\left({ }^{\circ}\right)\end{array}$ & $\begin{array}{l}\text { Positional Un } \\
\text { statistical }\left(^{\circ}\right)\end{array}$ & $\begin{array}{l}\text { ertainty }^{3} \\
\text { total }\left({ }^{\circ}\right)\end{array}$ & $\begin{array}{c}\text { Extension } \\
\mathrm{UL}^{4}\left({ }^{\circ}\right)\end{array}$ \\
\hline $0.06-0.3$ & 2246 & $(5.17 \pm 0.16) \times 10^{-7}$ & $(1.06 \pm 0.03) \times 10^{-10}$ & $266.407,-29.013$ & 0.045 & 0.050 & 0.2404 \\
\hline $0.3-3$ & 10522 & $(1.49 \pm 0.17) \times 10^{-7}$ & $(1.99 \pm 0.20) \times 10^{-10}$ & $266.394,-28.997$ & 0.005 & 0.009 & 0.1141 \\
\hline $3-10$ & 3618 & $(8.94 \pm 0.22) \times 10^{-9}$ & $(6.69 \pm 0.17) \times 10^{-11}$ & $266.406,-29.003$ & 0.005 & 0.009 & 0.0785 \\
\hline $10-500$ & 321 & $(1.23 \pm 0.11) \times 10^{-9}$ & $(3.45 \pm 0.35) \times 10^{-11}$ & $266.415,-29.010$ & 0.005 & 0.010 & 0.0478 \\
\hline $0.1-500$ & 14724 & $(2.83 \pm 0.08) \times 10^{-7}$ & $(3.26 \pm 0.05) \times 10^{-10}$ & & & & \\
\hline
\end{tabular}

$1 \sqrt{T S} \approx$ detection significance of the source in each energy range

${ }^{2} \mathrm{RA}$ and Dec. corresponding to the emission centroid in degrees

$368 \%$ positional uncertainty

$495 \%$ confidence level

Assuming a distance to 4FGL J1745.6-2859 of $8.2 \mathrm{kpc}$ (GRAVITY Collaboration et al., 2019) and isotropic emission, the energy flux of $(3.26 \pm 0.05) \times 10^{-10} \mathrm{erg} \mathrm{cm}^{-2} \mathrm{~s}^{-1}$ measured in the $100 \mathrm{MeV}$ to $500 \mathrm{GeV}$ energy range corresponds to a gamma-ray luminosity of $(2.61 \pm 0.05) \times 10^{36} \mathrm{erg} \mathrm{s}^{-1}$. This luminosity is comparable to the observed radio-toX-ray luminosity of Sgr $\mathrm{A} * \sim 10^{36} \mathrm{erg} \mathrm{s}^{-1}$ (Genzel et al., 2010). The implications of this similarity will be discussed in Section 4

\subsection{Position in different energy bands}

We used Fermipy's extension tool to constrain the point source location in each energy range. Figure 3.2 shows the dependence of the source location on energy, together with the radio position of Sgr A* as measured by the Very Long Baseline Array (Petrov et al. 2011) and locations of other potential gamma-ray emitters in the GC. For a version of this plot with the $95 \%$ confidence level positional uncertainty, see Appendix L. 


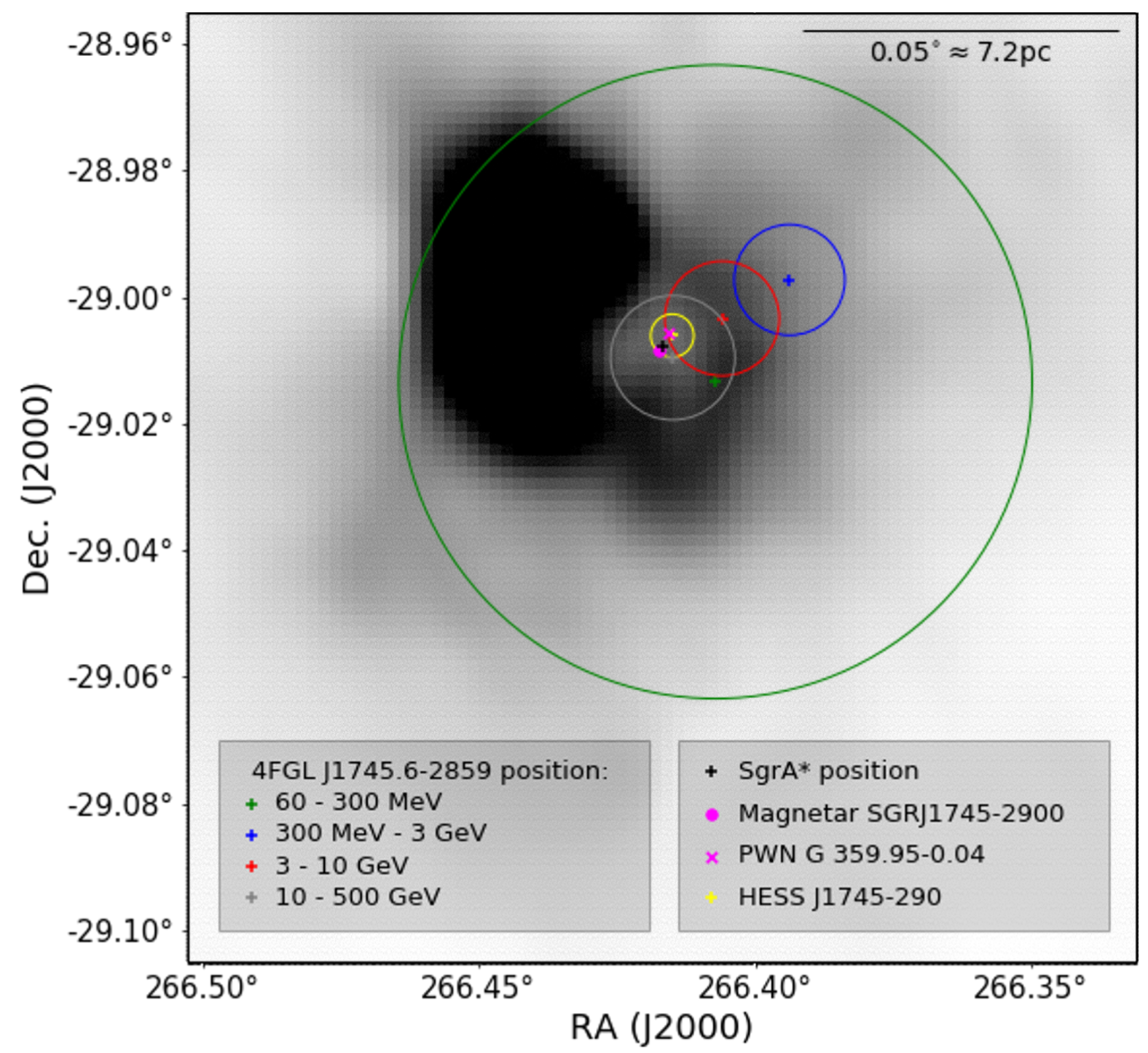

Figure 3.2: The position of the central source as a function of the energy range used in the analysis: green (60-300 MeV), blue (300 MeV-3 GeV), red (3-10 GeV) and gray (10-500 GeV). The circles represent the $68 \%$ positional uncertainty. The radio position of Sgr A* is indicated by the black cross. The positions of other gamma-ray-emitters in the GC are also indicated. The gray scale represents the $90 \mathrm{~cm}$ radio flux density map of Sagittarius A East (LaRosa et al., 2000), with darker color meaning brighter emission.

In Figure 3.2 we can see that the position uncertainty of the source in the lowest energy range is the largest. This is the result of a combination of factors: the broadening of Fermi LAT's PSF at lower energies, the energy dependence of the instrument's field of view and effective area, the central source's spectrum and the impact of the Galactic diffuse emission which is more prominent at lower energies.

The position of the source is consistent within $1 \sigma$ with Sgr A*in the energy ranges 60 $300 \mathrm{MeV}$ and 10-500 GeV. The gamma-centroid recedes from Sgr A* as the energy decrea- 
ses. This is also seen in Figure 3.3, which shows the distance between 4FGL J1745.6-2859 and Sgr A* as a function of energy.

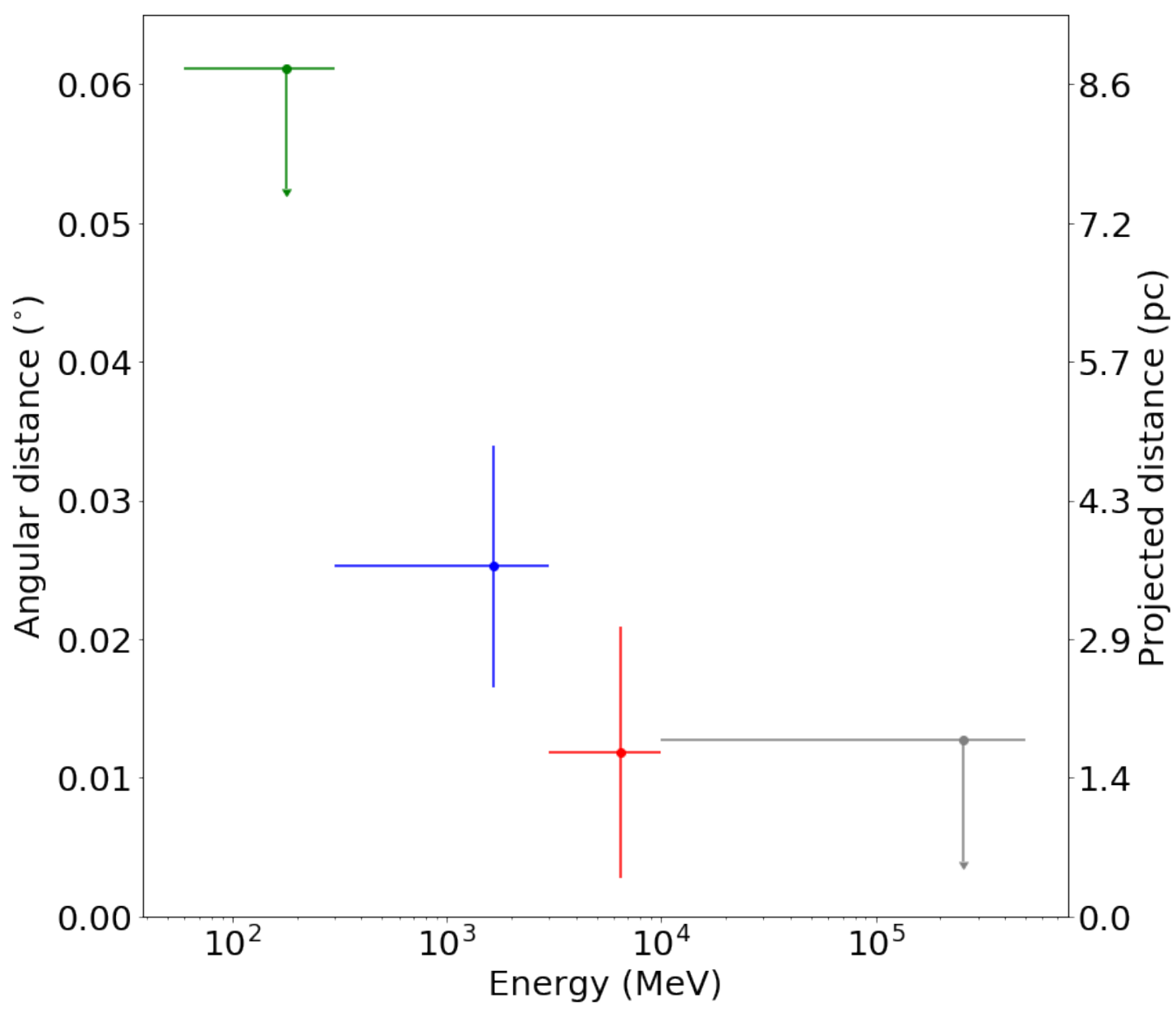

Figure 3.3: The distance between the central source and Sgr A* as a function of energy. Since the central source and Sgr A* are spatially coincident in the lowest and the highest energy bands, we show the ULs for these distances.

The positional uncertainties of the sources in the three highest-energy bands are on the order of $0 .^{\circ} 01$. This value is similar to the semi-major axis of the error ellipse at $68 \%$ confidence for 4FGL J1745.6-2859 in the 4FGL Catalog. It is smaller than the pixel size used in the analysis of these energy bands $\left(0{ }^{\circ} 08\right)$. We performed a simple statistical simulation to see if we could reproduce results with positional uncertainty smaller than the bin size. We generate a mock source with a size smaller than the bin size. We then computed the uncertainty and found that it can be smaller than the bin size, therefore this result is not unexpected.

We also tested the likelihood of the source being extended versus a point source using the extension tool. Figure 3.4 shows the $95 \%$ upper limits on the spatial extension of the 
source for each energy range. Their numerical values can be found in the last column of Table 3.1 .

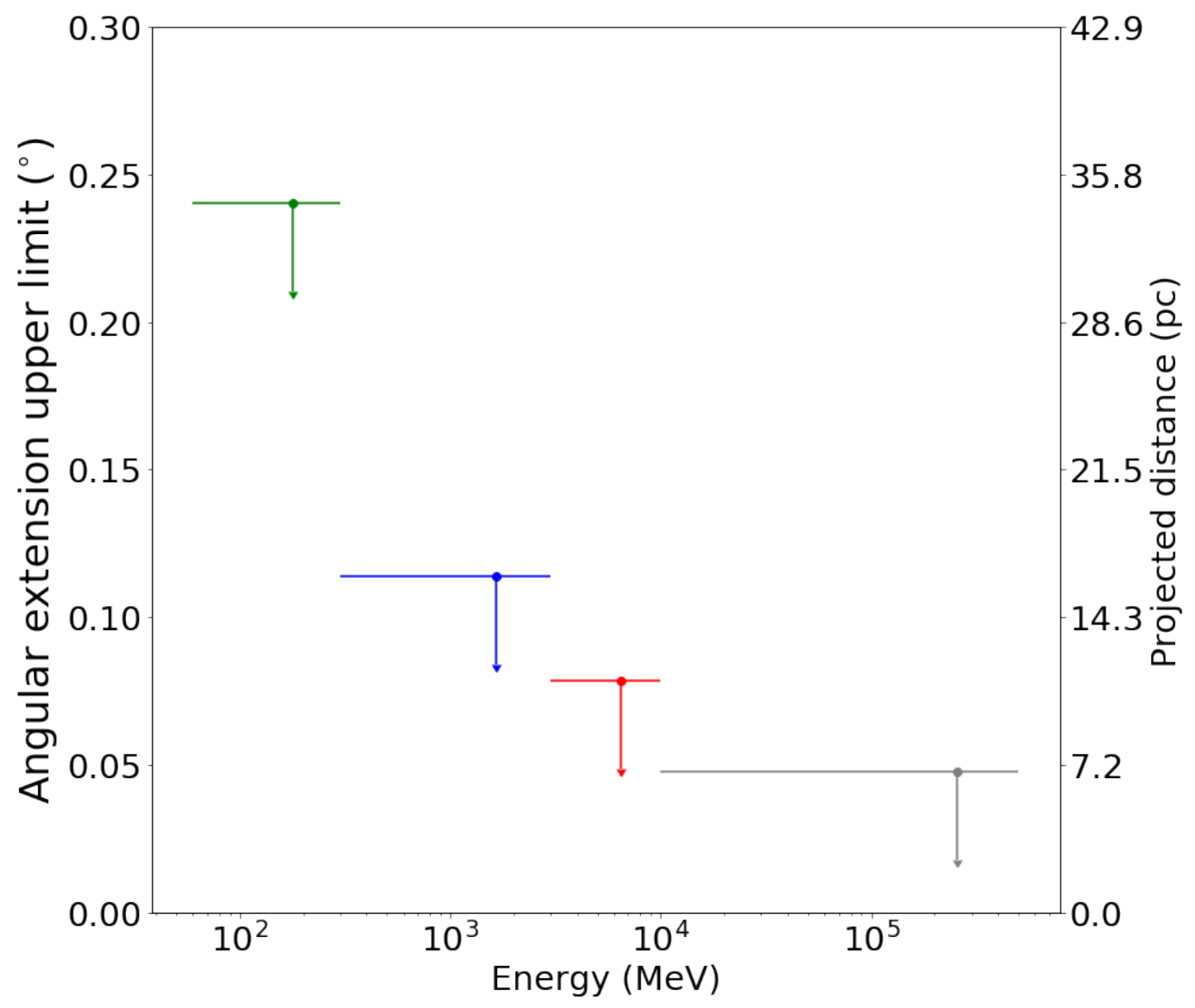

Figure 3.4: Upper limit on the spatial extension of the central source as a function of energy. Upper limits correspond to the $95 \%$ confidence level.

In Section 2.4 we explained the difficulties in assessing the extension of the central source in the energy bands we are studying, so we only report the ULs on the extension. We find no conclusive evidence for any spatially extended emission from the central source, for the following reasons. We start by comparing these ULs with the positional uncertainties of other sources in the 4FGL Catalog. The median positional uncertainties for high-latitude cataloged point-sources with $25<T S<100$ is of $\sim 0 .{ }^{\circ} 08$ (Figure 3.5). This value is larger than the ULs we obtained for the central source's extension for the two highest energy bands. It is important to mention that the 4FGL Catalog was constructed in the energy range between $50 \mathrm{MeV}$ and $1 \mathrm{TeV}$, a band much larger than the ones we are using and that covers energies in which Fermi LAT's PSF is much better than in our low energy band. In order to have a fair comparison between the extension UL in the low energy band and the 
positional uncertainties of known point sources, we refer to the first catalog of Fermi-LAT sources below $100 \mathrm{MeV}$ (1FLE, Principe et al.2018). In the 1FLE, the average and median positional uncertainties (95\% confidence level) are of $0^{\circ} .245$ and $0^{\circ} .246$, respectively, which are larger than the extension UL we obtained in the lowest energy band of this work. The ULs we obtained, then, are compatible with the positional uncertainties of cataloged point sources observed with Fermi-LAT in similar energy ranges.

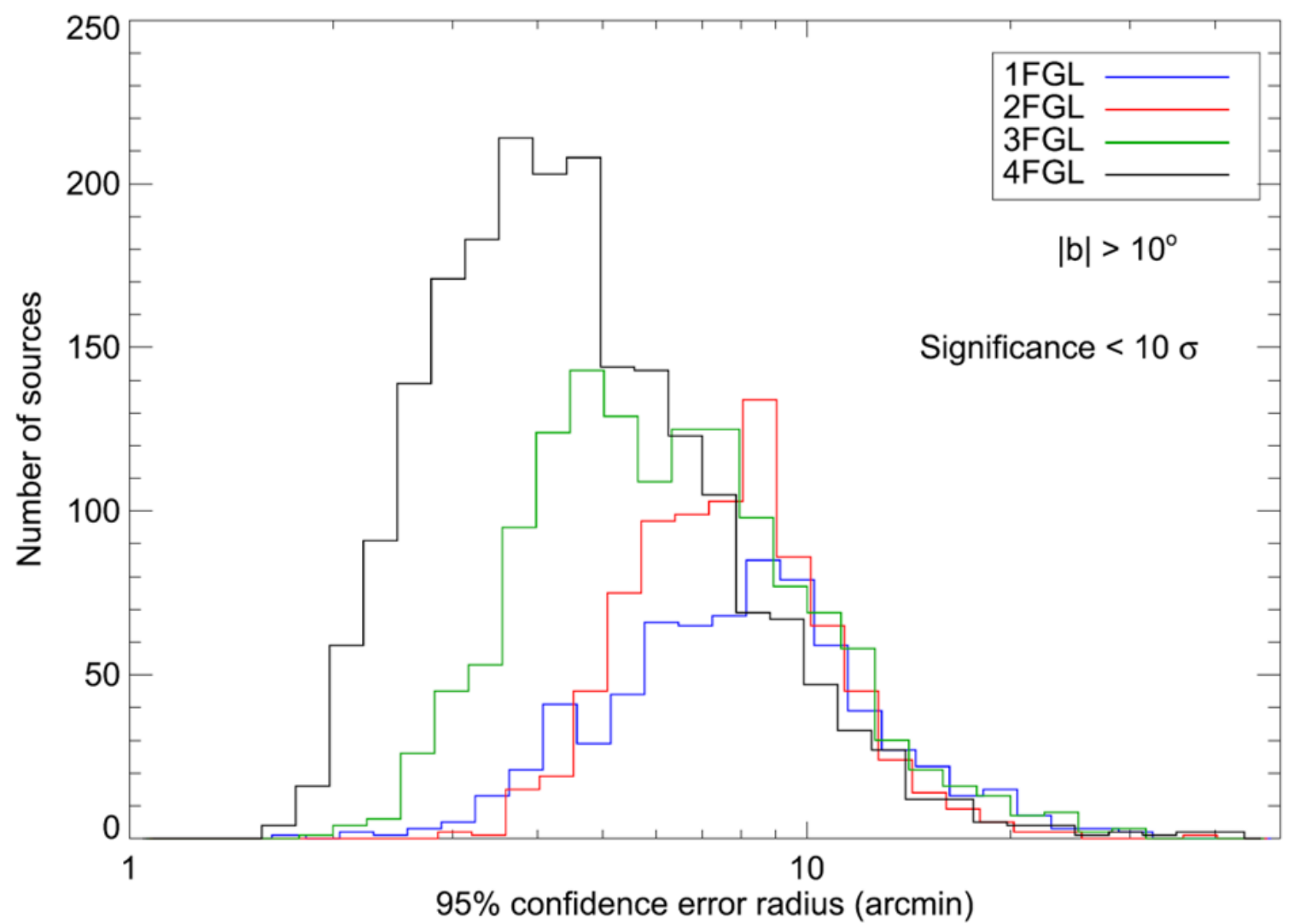

Figure 3.5: Distributions of the $95 \%$ confidence error radii for high-latitude sources with $T S<100$ in the four versions of the Fermi Catalogues. The distribution for the 4FGL has median $\sim 0^{\circ} .08$.

\subsection{Light curves}

Here we present the LCs obtained in Section 2.5.1. We start with the 15 days bins LCs, followed by the LCs created using the tighter energy bands. We present the 45 days bins LCs, in Appendix $\mathrm{K}$. 


\subsubsection{5 days bins, Universal Model}

In the upper and bottom panels of Figure 3.6 we show, respectively, the 15 days bins energy and the photon flux LCs in the $100 \mathrm{MeV}-500 \mathrm{GeV}$ energy band, compared with the average fluxes. In all the LCs presented in this thesis, the error bars correspond to the $68 \%$ confidence level uncertainty. In the time bins with $T S<16$, we show the $95 \%$ confidence level UL. In Appendix $\mathrm{M}$ we exhibit the TS values of every LC presented in this work.

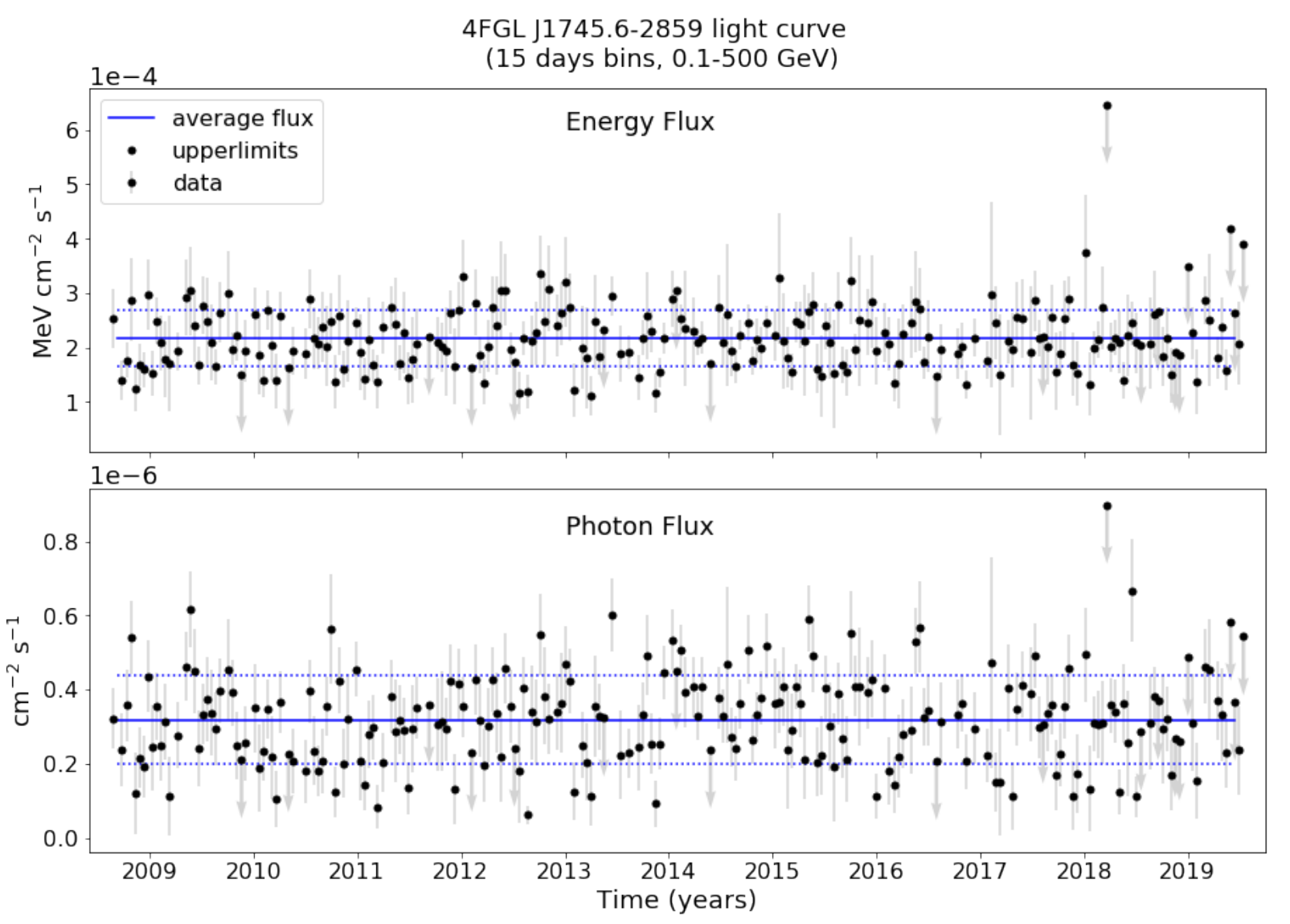

Figure 3.6: These are the same 15 days LCs of 4FGL J1745.6-2859 in the $100 \mathrm{MeV}-500 \mathrm{GeV}$ energy band shown in Figure 2.12 with the addition of the mean flux (blue line). The dotted blue lines correspond to the $1 \sigma$ uncertainty of the average flux.

In most of the time bins of both panels of Figure 3.6 the flux is compatible with the average flux. This is an indication that the source is in a quiescent state most of the time, but it does not rule out the hypothesis of variability. We decided to use the rolling average of the flux to look for bins with fluxes significantly different than the neighboring bins. In the upper and bottom panels of Figure 3.7 we plot the energy and photon flux LCs with the 5 bins ( $=75$ days in total) rolling average. The rolling average values are plotted on 
the date corresponding to the center of the 5 bins.

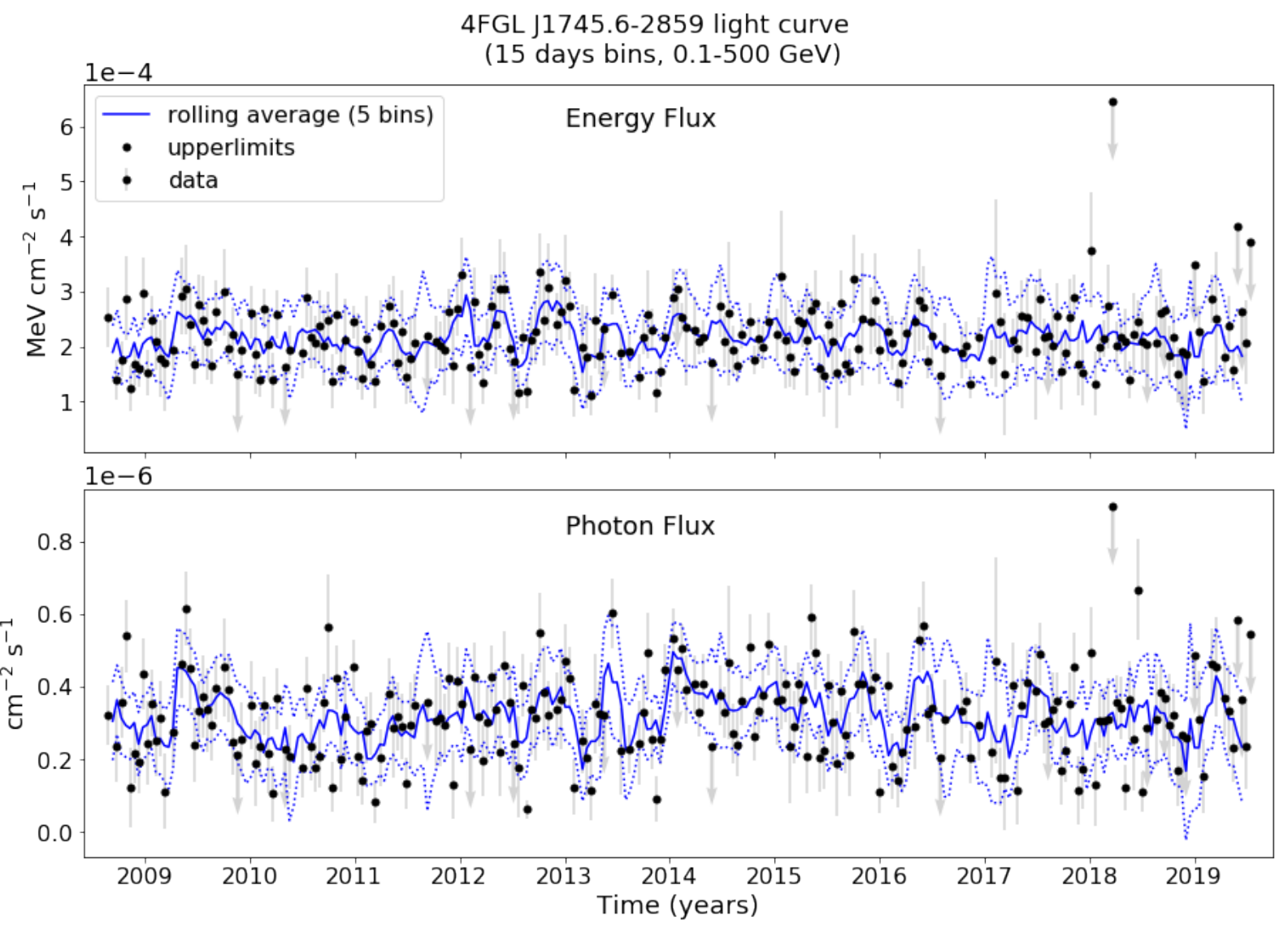

Figure 3.7: These are the same 15 days LCs of 4FGL J1745.6-2859 in the $100 \mathrm{MeV}-500 \mathrm{GeV}$ energy band shown in Figure 2.12 with the addition of the rolling average flux (blue line) of 5 bins. The dotted lines correspond to the $2 \sigma$ uncertainty of the rolling average flux.

Of the whole 204 data points (not considering the ULs), in the energy flux LC (upper panel of Figure 3.7), only one is barely not compatible with the $2 \sigma$ uncertainty of the 5 bins rolling average. The energy flux in this bin is smaller than the rolling average. The same method applied to the photon flux LC (bottom panel of Figure 3.7) reveals 7 (11) bins with fluxes only slightly above (below), the rolling average $\pm 2 \sigma$.

To check if the variability observed in these LCs arises from a random process, we tested if the flux distribution is compatible with a Gaussian, representative of a normal random process, or a log-normal, consistent with a flaring source. In Figure 3.8 we show the histograms of the flux distribution. The energy flux distribution is shown in panel a) and the photon flux in panel b). Both distributions seen to be compatible with a Gaussian fit, shown by the red curves. In panel c) we plot the the logarithm of the photon flux distribution. The red curve in panel c) corresponds to a log-normal fit to the data (which 
is simply a Gaussian in log space). The data does not seem to be compatible with a log-normal distribution.

In Tables 3.2 and 3.3 we show the results of 3 different tests used to quantify whether the data follow a Normal distribution. The Shapiro-Wilk and the D'Agostino's $\mathrm{K}^{2}$ test could not reject the null hypothesis that the Energy and the Photon fluxes follow a normal distribution. But it was possible to reject the log-normal distribution with a significance better than $95 \%$. The same results were obtained with the Anderson-Darling normality test (Table 3.3). It was also not possible to reject the hypothesis that the Energy and Photon flux LCs are normally distributed even if we were willing to accept confidence levels as low as $85 \%$. However, we could reject the null hypothesis that it is log-normally distributed with a confidence level of $99 \%(\sim 3 \sigma)$.

The results shown in Tables 3.2 and 3.3 indicate that 4FGL J1745.6-2859's flux distributions are compatible with Gaussian distributions, which means that the variability likely arises from a random process. It is incompatible with a log-normal distribution, expected in the case of a flaring source.

Table 3.2 - Results of the Shapiro-Wilk and the D'Agostino's K ${ }^{2}$ normality tests for the 15 days LCs in the $0.1 \mathrm{MeV}-500 \mathrm{GeV}$ energy range. The flux distribution is compatible with a Gaussian fit.

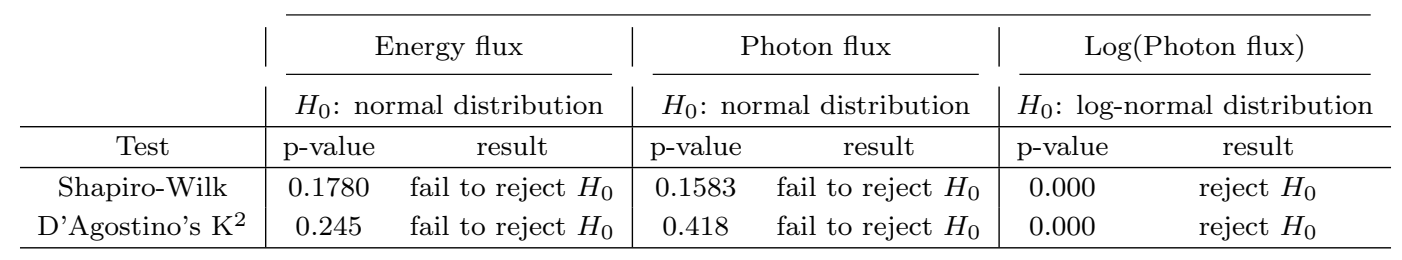

Table 3.3 - Results of the Anderson-Darling normality test for the 15 days LCs in the $0.1 \mathrm{MeV}-500 \mathrm{GeV}$ energy range. The flux distribution is compatible with a Gaussian fit.

\begin{tabular}{|c|c|c|c|c|c|c|c|c|c|}
\hline & \multicolumn{3}{|c|}{ Energy flux } & \multicolumn{3}{|c|}{ Photon flux } & \multicolumn{3}{|c|}{ Log(Photon flux) } \\
\hline Significance & \multicolumn{3}{|c|}{$H_{0}:$ normal distribution } & \multicolumn{3}{|c|}{$H_{0}:$ normal distribution } & \multicolumn{3}{|c|}{$H_{0}:$ log-normal distribution } \\
\hline level & statistic & crit. value & result & statistic & crit. value & result & statistic & crit. value & result \\
\hline 15 & & 0.565 & fail to reject $H_{0}$ & & 0.565 & fail to reject $H_{0}$ & & 0.565 & reject $H_{0}$ \\
\hline 10 & & 0.644 & fail to reject $H_{0}$ & & 0.644 & fail to reject $H_{0}$ & & 0.644 & reject $H_{0}$ \\
\hline 5 & 0.4022 & 0.772 & fail to reject $H_{0}$ & 0.4081 & 0.772 & fail to reject $H_{0}$ & 3.331 & 0.772 & reject $H_{0}$ \\
\hline 2.5 & & 0.901 & fail to reject $H_{0}$ & & 0.901 & fail to reject $H_{0}$ & & 0.901 & reject $H_{0}$ \\
\hline 1 & & 1.072 & fail to reject $H_{0}$ & & 1.072 & fail to reject $H_{0}$ & & 1.072 & reject $H_{0}$ \\
\hline
\end{tabular}

Finally, we tested the presence of a global trend in the $\sim 11.3$ years of data collection. We simply fitted linear functions to the observed data. The results are in Figure 3.9. In Table 3.4 we show the linear and angular coefficients (with their uncertainties) obtained in 

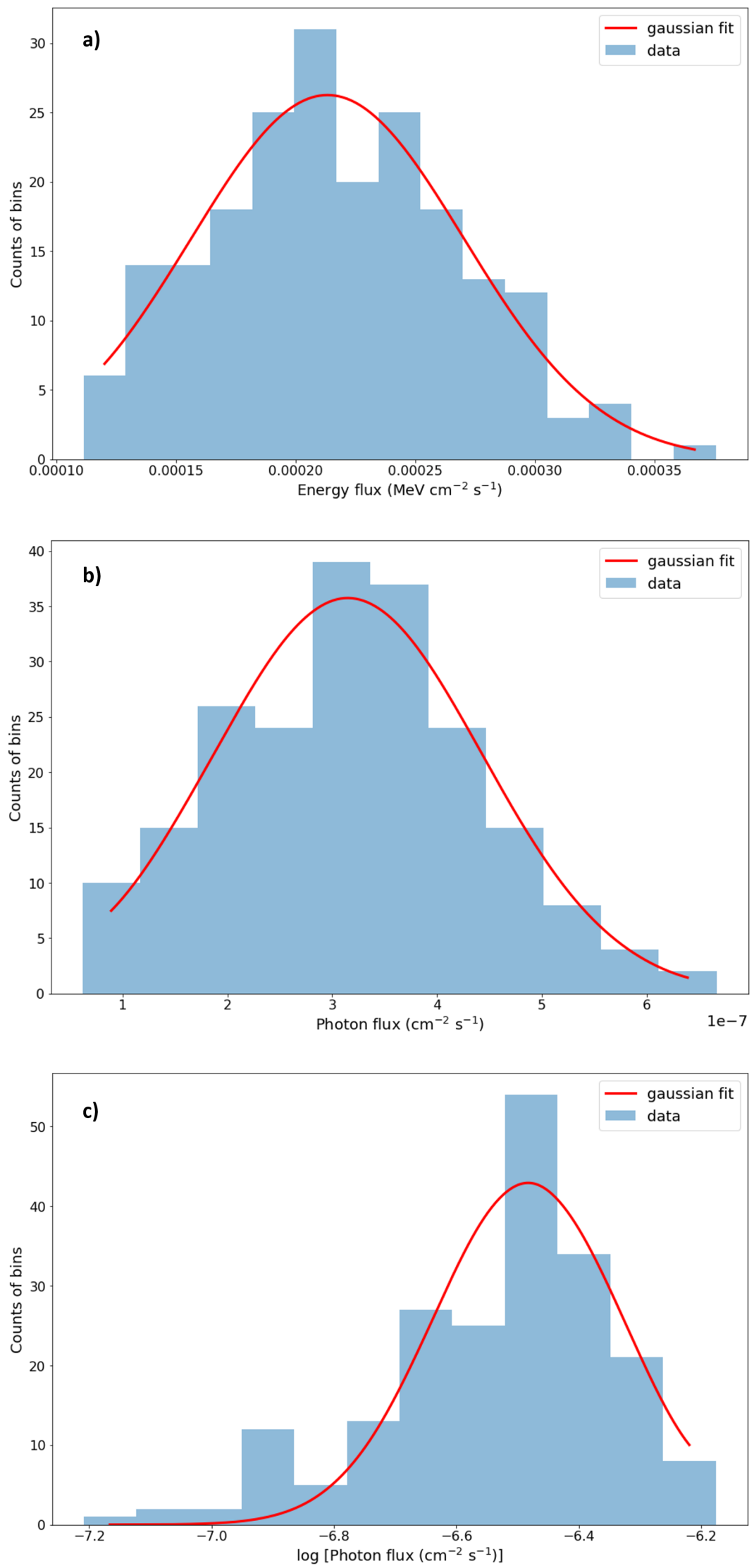

Figure 3.8: The flux distribution for the 15 days bins LCs in the $0.1 \mathrm{MeV}-500 \mathrm{GeV}$. We show the distributions for the energy flux, the photon flux and the logarithm of the photon flux in panels a), b) and c), respectively. The red curve corresponds to the Gaussian fits to the distribution (a log-normal fit in panel c)). A Gaussian fit is clearly preferable for the fluxes distribution (see Tables 3.2 and 3.3 . 
these fits. Both LCs are compatible with constant flux for the duration of the observations (angular coefficient compatible with 0 ).
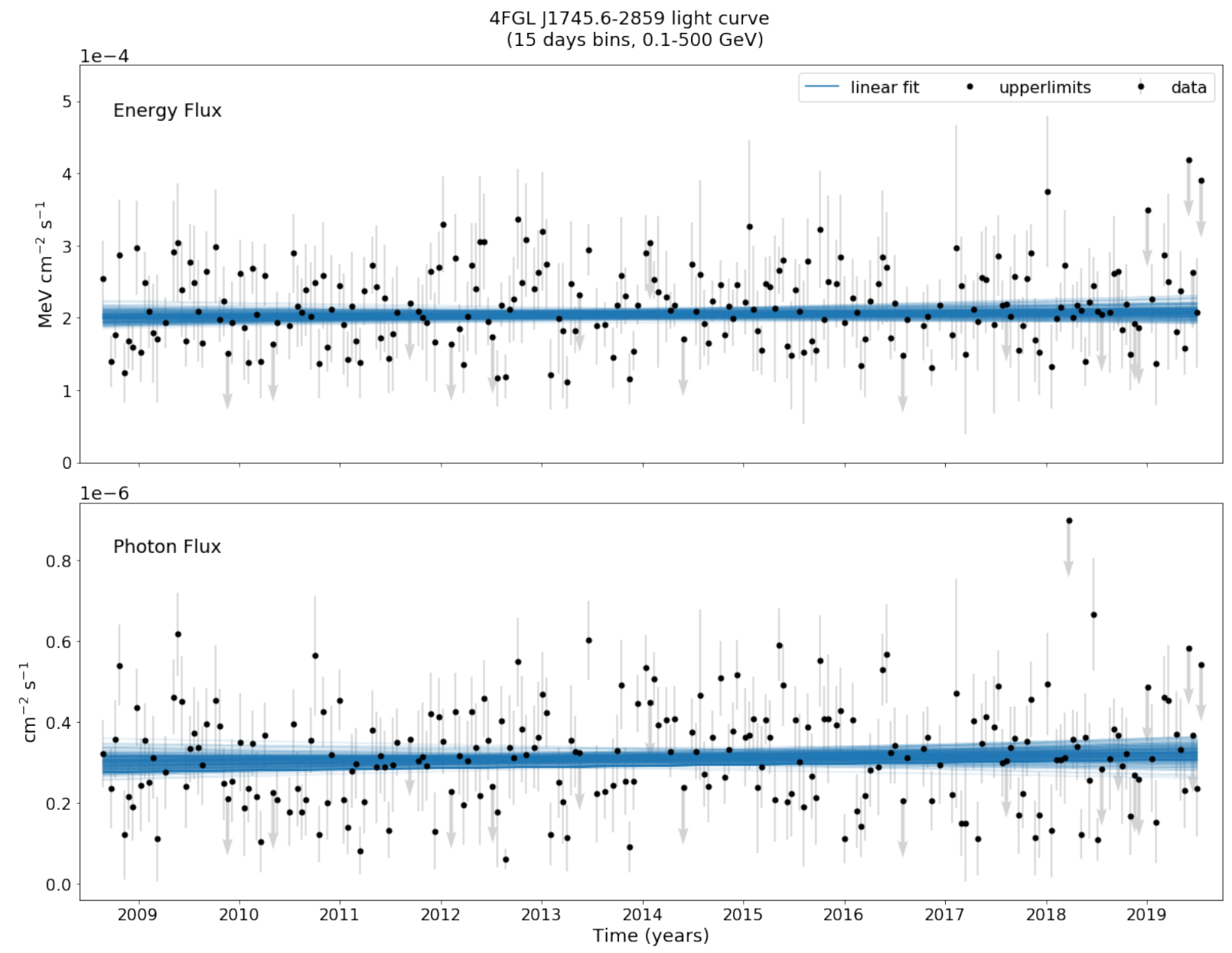

Figure 3.9: These are the same 15 days LCs of 4FGL J1745.6-2859 in the $100 \mathrm{MeV}-500 \mathrm{GeV}$ energy band shown in Figure 2.12 fitted with linear functions. We plotted in blue 200 randomized selected MCMC linear fits to the data.

Table 3.4 - Results of a linear fit to the $100 \mathrm{MeV}-500 \mathrm{GeV}$ energy range 15 days bins LCs.

\begin{tabular}{ccc}
\hline \hline Parameter & \multicolumn{2}{c}{ Light curve } \\
& Energy flux & Photon flux \\
\hline Angular coefficient & $(2.5 \pm 4.2) \times 10^{-14}$ & $(4.9 \pm 9.3) \times 10^{-17}$ \\
Linear coefficient & $(1.93 \pm 0.18) \times 10^{-4}$ & $(2.9 \pm 0.4) \times 10^{-7}$ \\
\hline
\end{tabular}

The results obtained for the 15 days bins LCs are compatible with 4FGL J1745.6-2859's flux being generated by a normal random process. Very few time bins have a flux significantly incompatible with the average flux. Also, there is no signal of a global trend in the data. In Section 4.2 we discuss these results in the context of Sgr A* as the source of the gamma-ray emission. 


\subsubsection{Separate energy intervals}

We also created LCs for the models generated from the Universal model. We created 90-days bins LCs in the $300 \mathrm{MeV}-3 \mathrm{GeV}$ and in the $3-10 \mathrm{GeV}$ energy bands. For the 10-500 GeV energy band we used 180 days time bins because of the low number of photon counts at higher energies, in order to have bins with $T S>16$. In Appendix $\mathbb{M}$ we exhibit the TS values of these LCs.

We start with the LCs accompanied with the average flux. We show the energy and the photon flux LCs in growing order of energy in Figures 3.10 to 3.12. As in the 100 $\mathrm{MeV}-500 \mathrm{GeV}$ LCs, very few points are incompatible within $1 \sigma$ with the average flux.

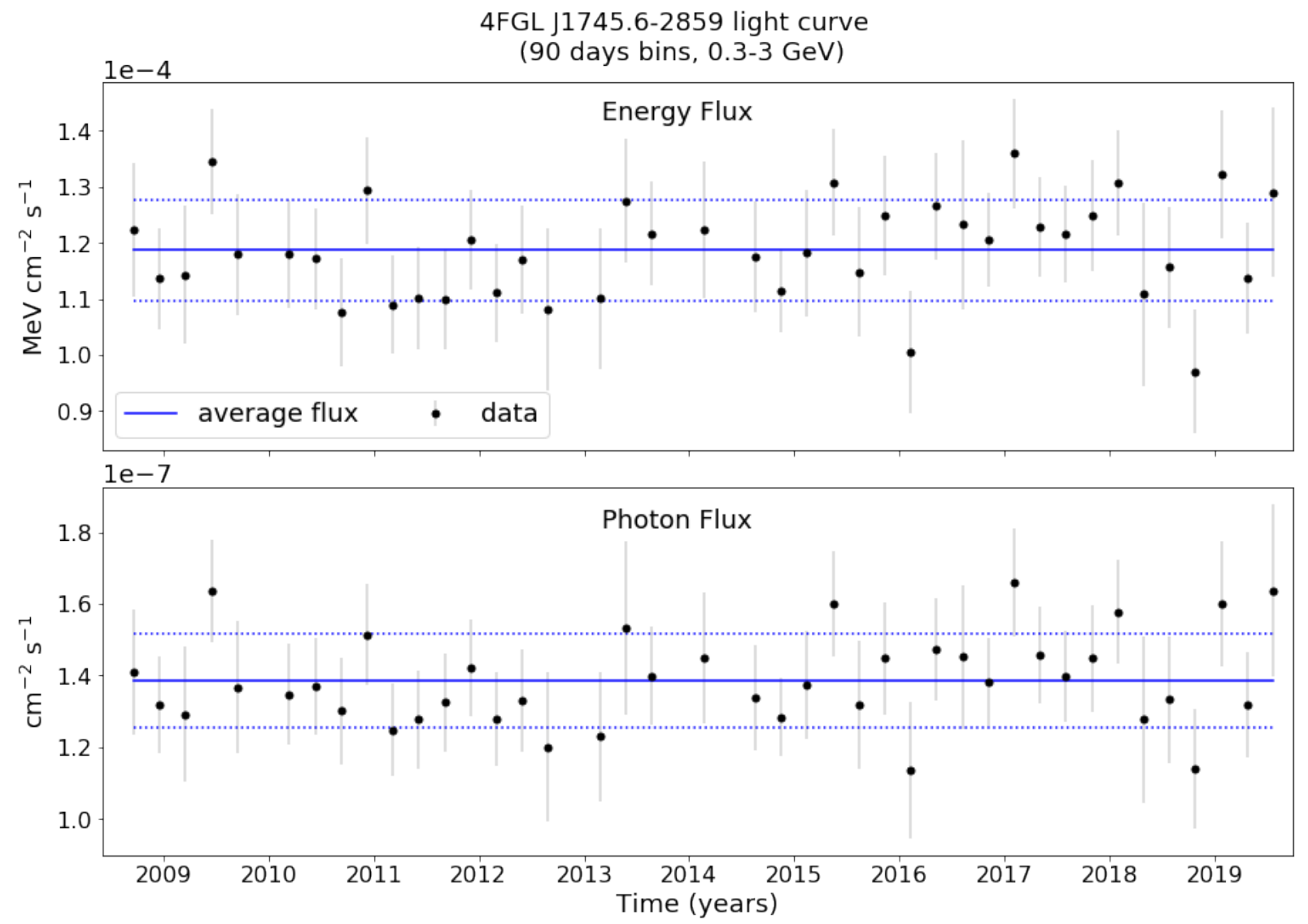

Figure 3.10: The 90 days LCs of 4FGL J1745.6-2859 in the $300 \mathrm{MeV}-3 \mathrm{GeV}$ energy band with the addition of the mean flux (blue line). The dotted blue lines correspond to the $1 \sigma$ uncertainty of the average flux. 

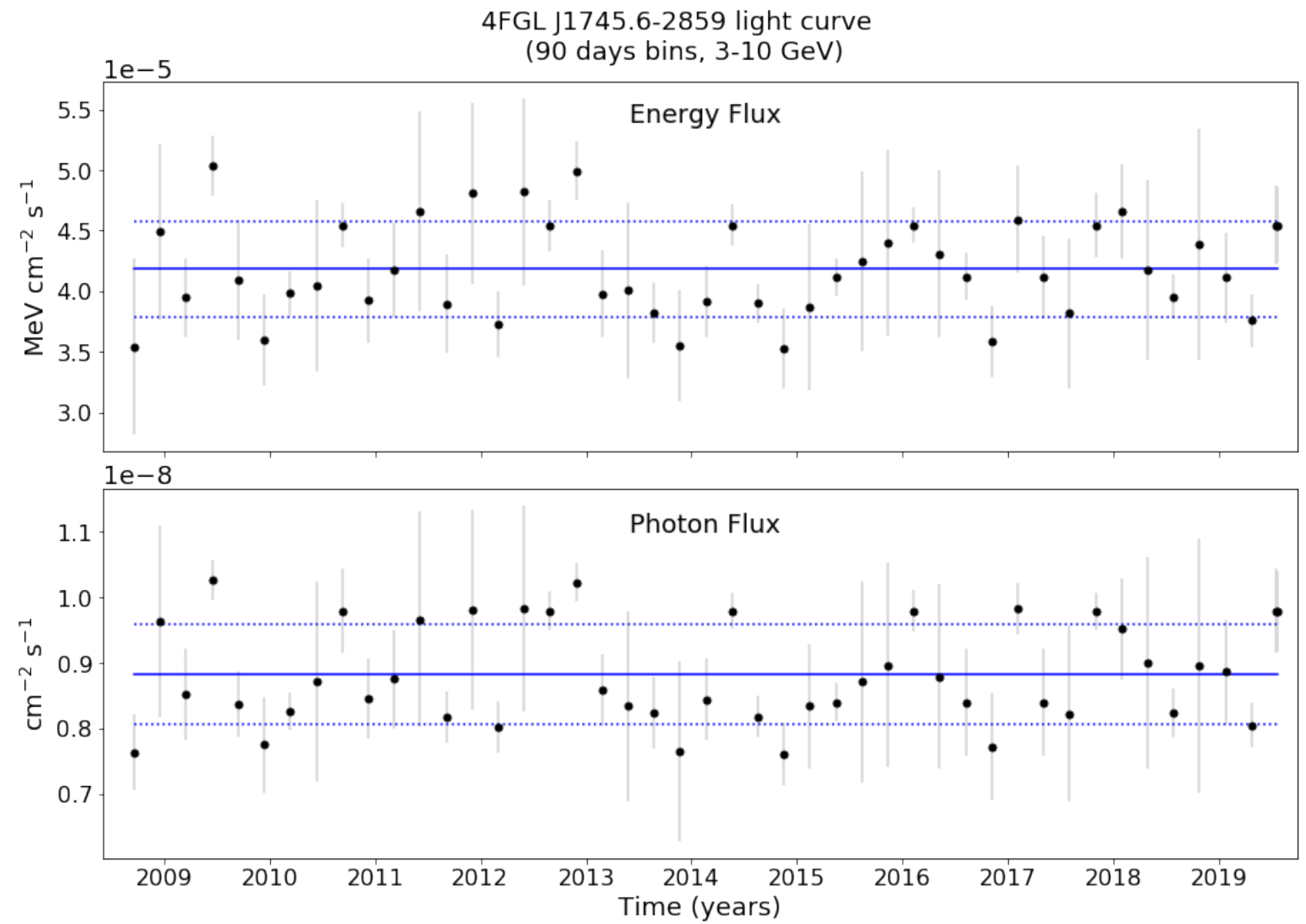

Figure 3.11: The 90 days LCs of 4FGL J1745.6-2859 in the 3-10 GeV energy band with the addition of the mean flux (blue line). The dotted blue lines correspond to the $1 \sigma$ uncertainty of the average flux. 


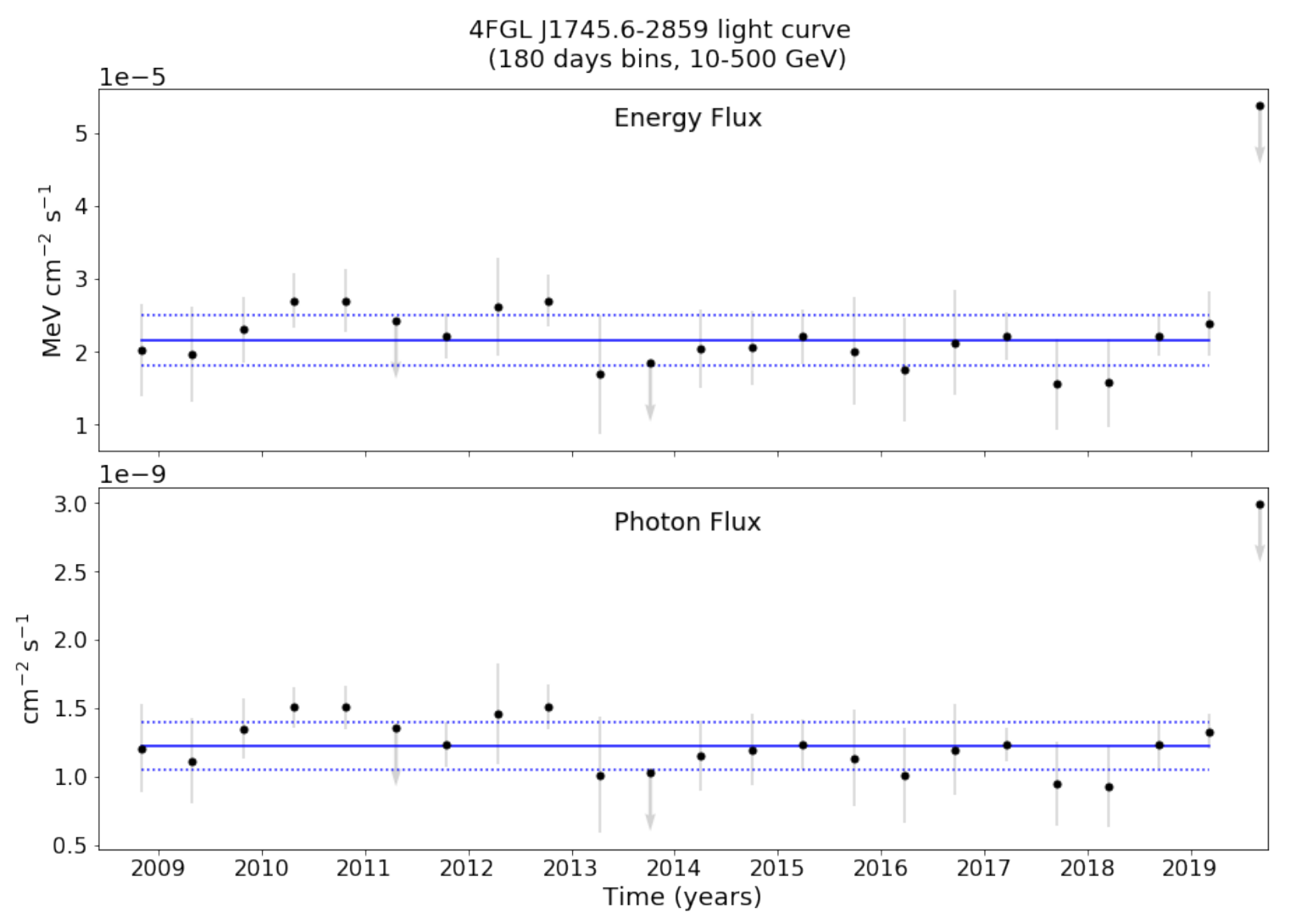

Figure 3.12: The 180 days LCs of 4FGL J1745.6-2859 in the 10-500 GeV energy band with the addition of the mean flux (blue line). The dotted blue lines correspond to the $1 \sigma$ uncertainty of the average flux.

We also compared the fluxes with their 5 bins rolling average. The results are presented in Figures 3.13 to 3.15 . Once again, with very few exceptions, the data points are compatible within $2 \sigma$ with the rolling average fluxes. The exceptions are 2 data points, from a total of 45, in the 90 days bins LCs in the 3-10 GeV energy band (Figure 3.14 at $\mathrm{t} \approx 2009.5$ and $\mathrm{t} \approx 2014.5)$. They are just barely outside the $2 \sigma$ band with the 5 bins rolling average. It is unlikely that they represent a flare from 4FGL J1745.6-2859 since this behavior was not detected in other energy bands nor in the better time-resolved LCs with 15 days bins. 

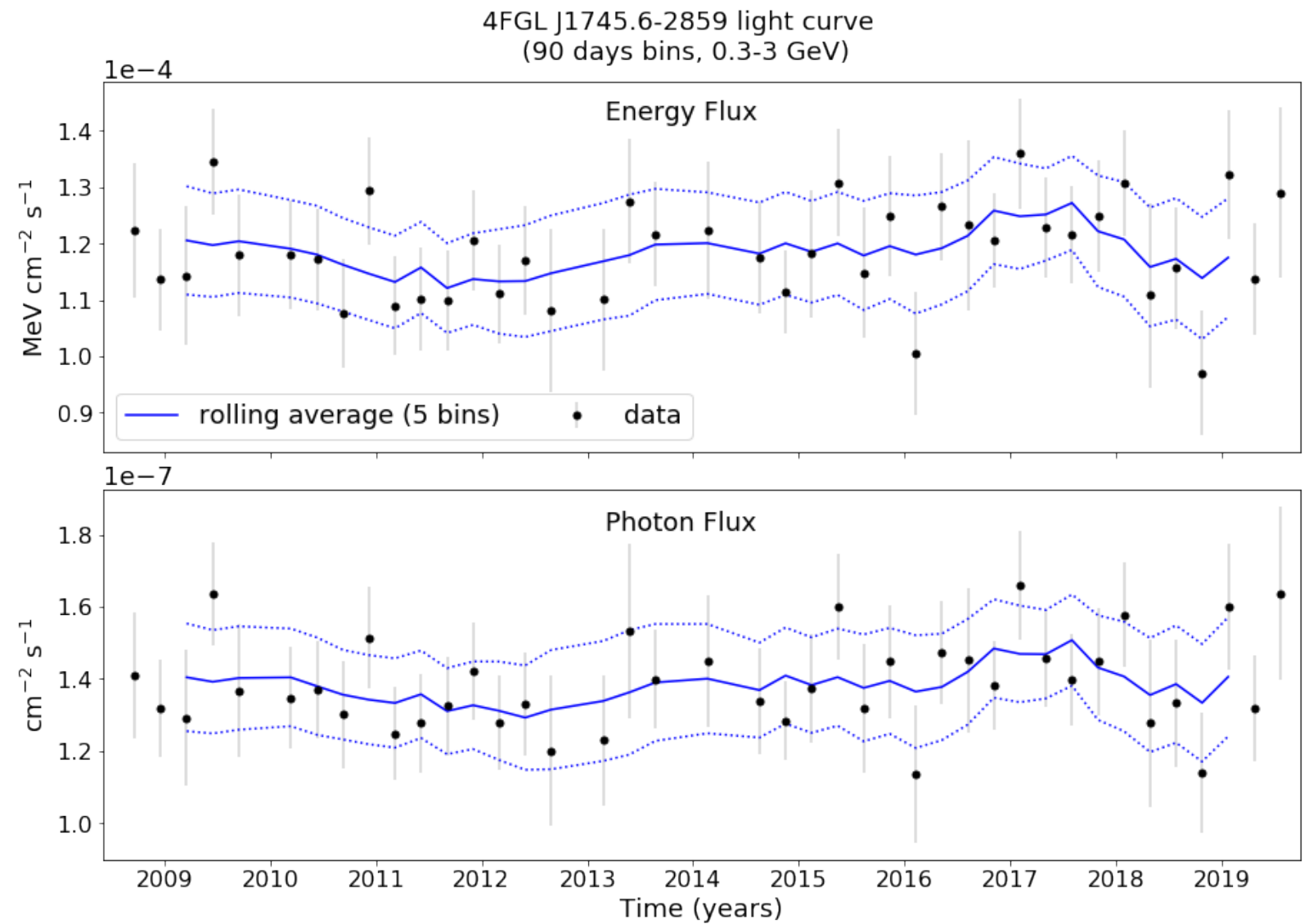

Figure 3.13: The 90 days LCs of 4 FGL J1745.6-2859 in the $300 \mathrm{MeV}-3 \mathrm{GeV}$ energy band with the addition of the rolling average flux of 5 bins (blue line). The dotted lines correspond to the $2 \sigma$ uncertainty of the rolling average flux. 

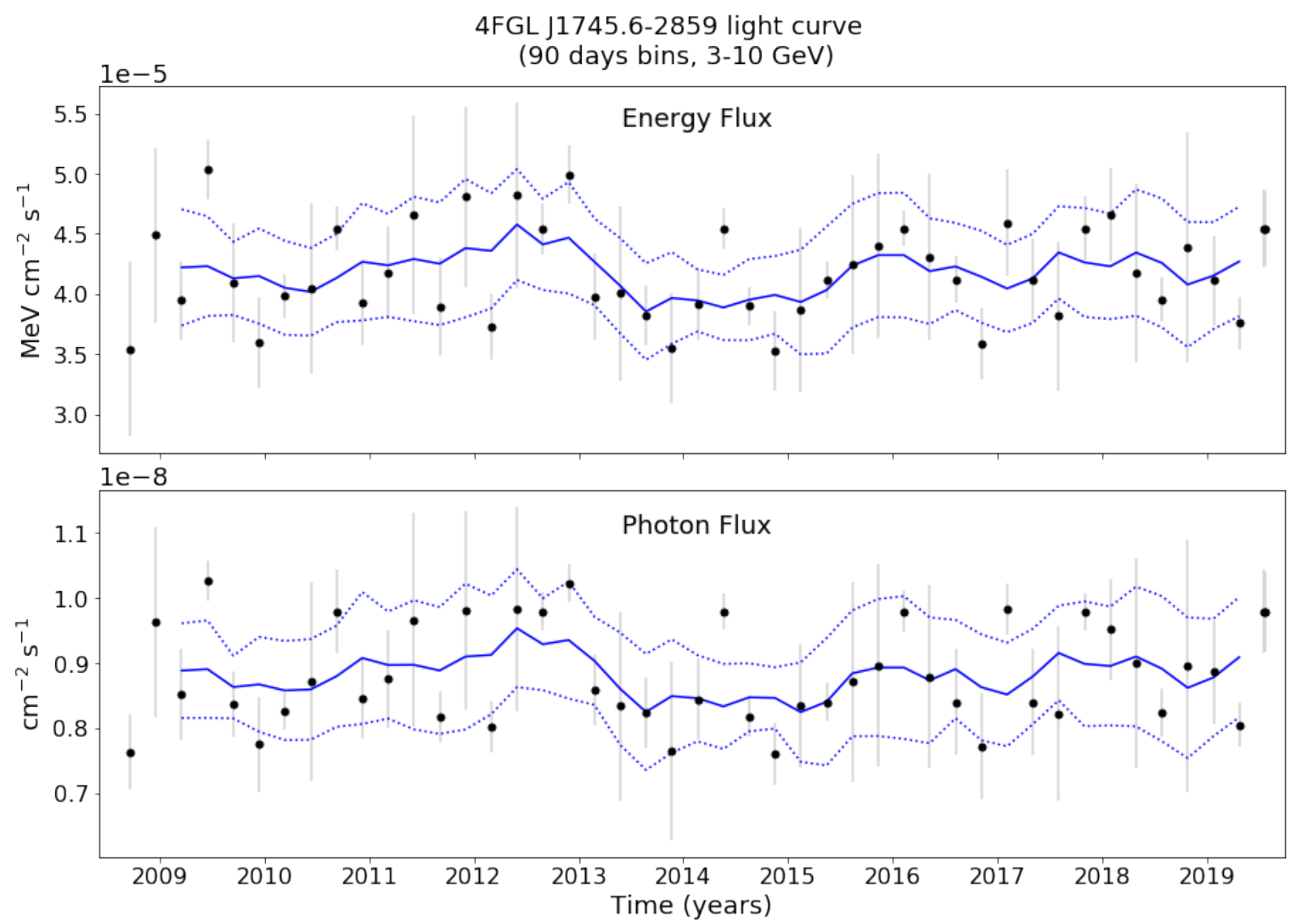

Figure 3.14: The 90 days LCs of 4FGL J1745.6-2859 in the 3-10 GeV energy band with the addition of the rolling average flux of 5 bins (blue line). The dotted lines correspond to the $2 \sigma$ uncertainty of the rolling average flux. 


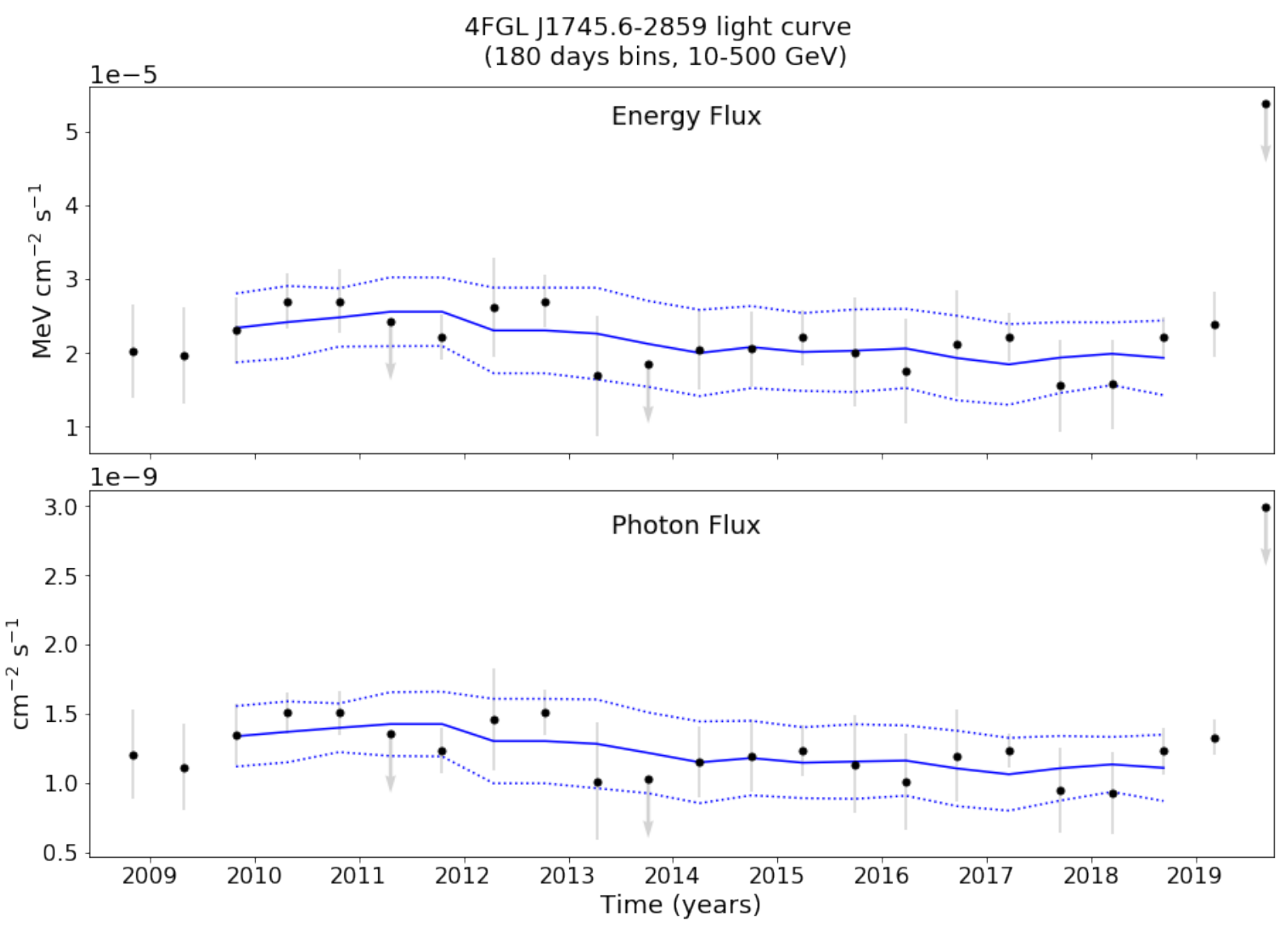

Figure 3.15: The 180 days LCs of 4FGL J1745.6-2859 in the 10-500 GeV energy band with the addition of the rolling average flux of 5 bins (blue line). The dotted lines correspond to the $2 \sigma$ uncertainty of the rolling average flux.

The plots shown in Figures 3.10 to 3.15 indicate that 4FGL J1745.6-2859's emission is also constant in time even in the tighter energy bands. We fitted linear functions to these LCs. Here we report the results, in Figures 3.16 to 3.18. In Table 3.5 we show the linear and angular coefficients obtained in these fits. With one exception, the LCs are compatible with constant flux for the duration of the observations since their angular coefficient is compatible with 0 . The exception is the photon flux LC in the $10-500 \mathrm{GeV}$, whose angular coefficient, although not compatible with 0, is very small (on the order of $\left.-10^{-19}\right)$ 

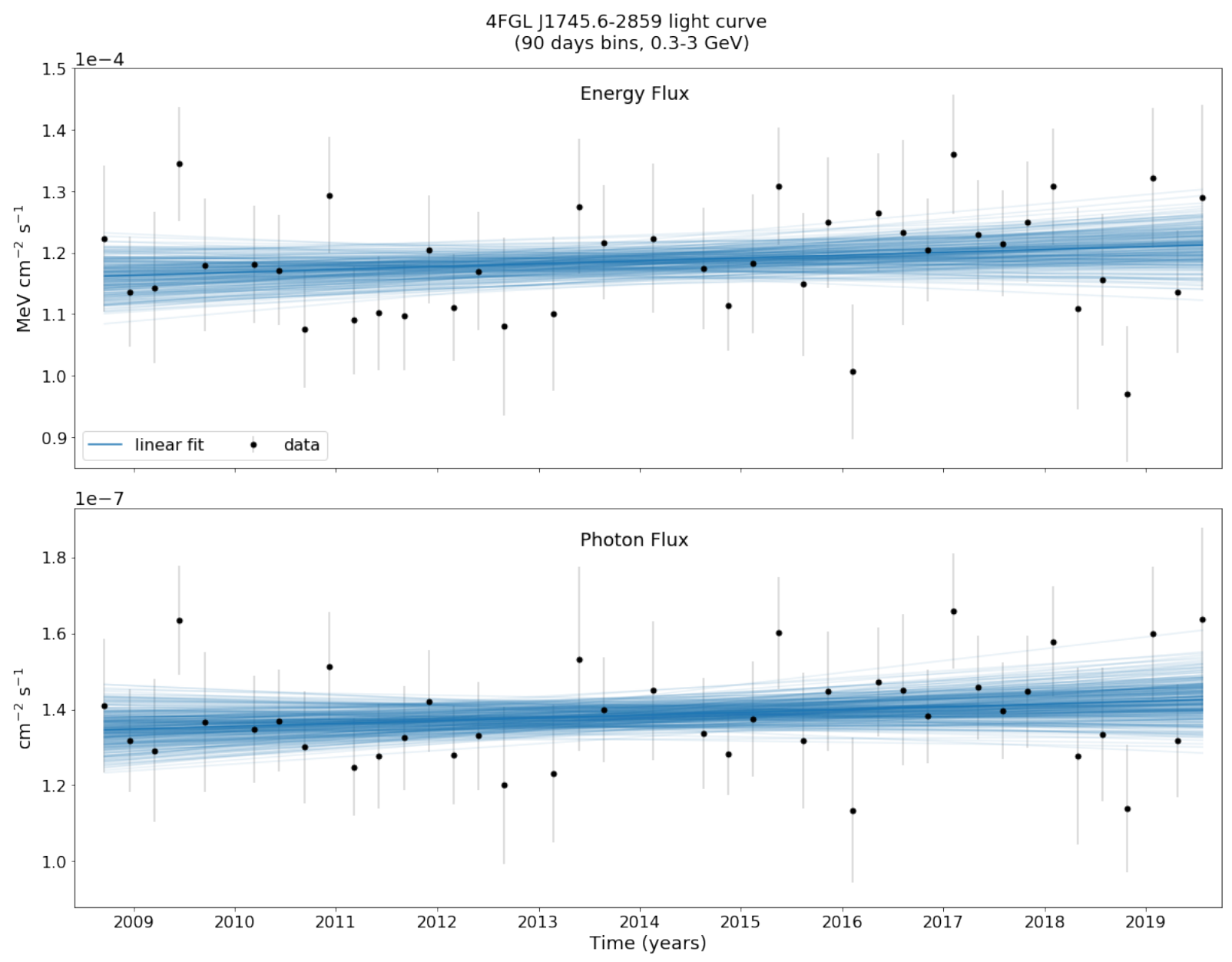

Figure 3.16: Linear fit to the 90 days bins LCs of 4FGL J1745.6-2859 in the $300 \mathrm{MeV}-3 \mathrm{GeV}$ energy band. We plotted in blue 200 randomized selected MCMC linear fits to the data. 

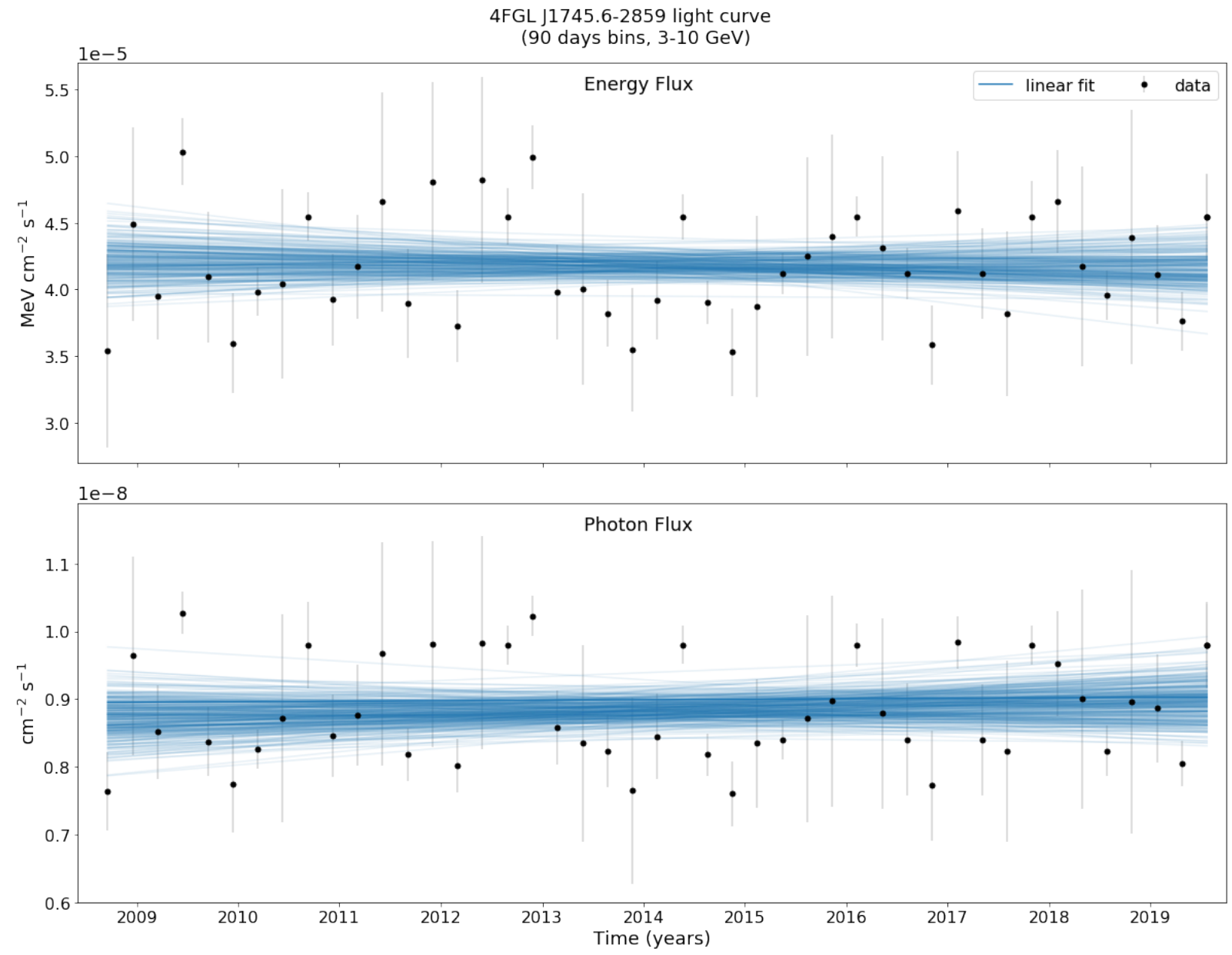

Figure 3.17: Linear fit to the 90 days bins LCs of 4FGL J1745.6-2859 in the 3-10 GeV energy band. We plotted in blue 200 randomized selected MCMC linear fits to the data. 


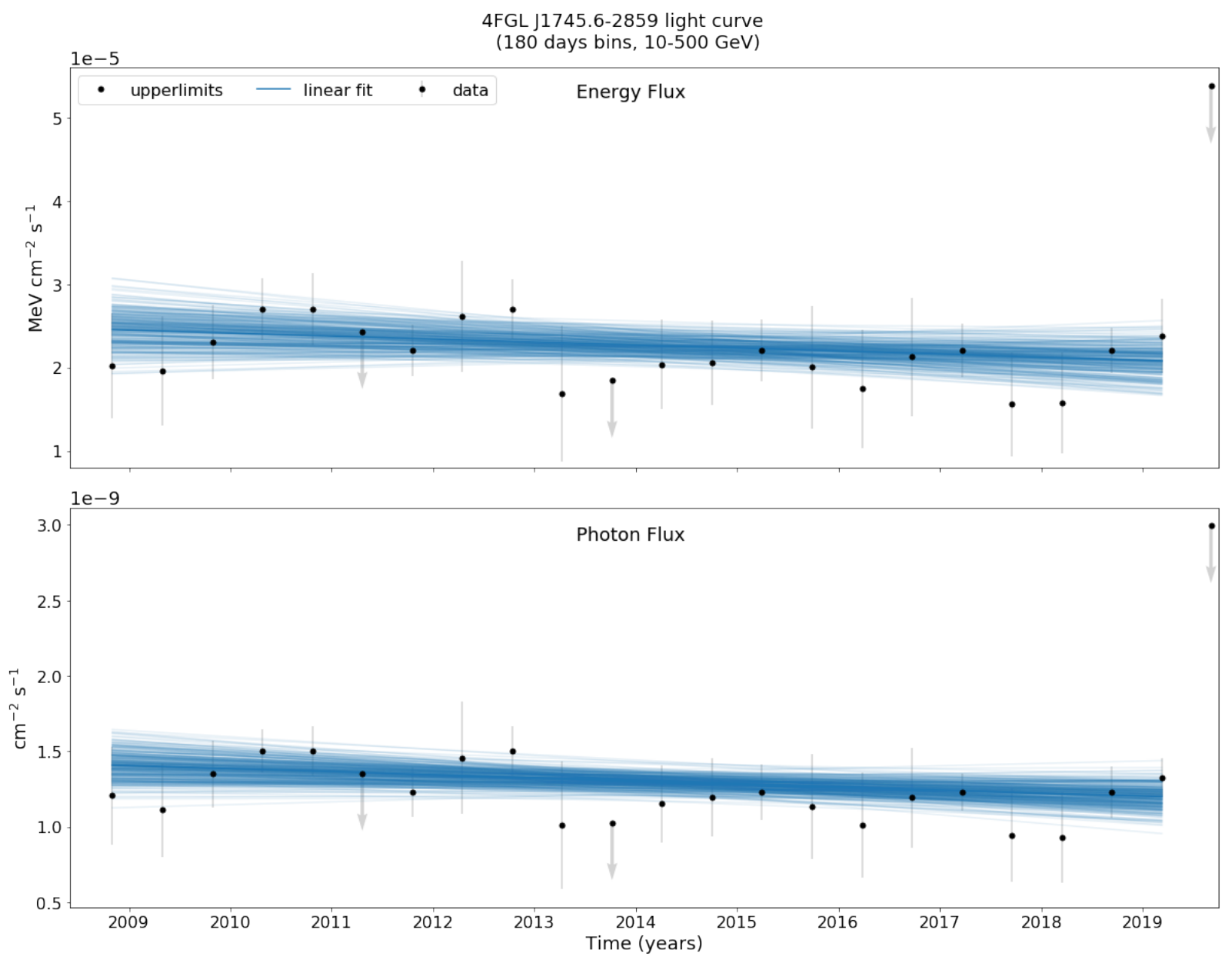

Figure 3.18: Linear fit to the 180 days bins LCs of 4FGL J1745.6-2859 in the 10-500 GeV energy band. We plotted in blue 200 randomized selected MCMC linear fits to the data.

Table 3.5 - Results of linear fits to the $300 \mathrm{MeV}-3 \mathrm{GeV}, 3-10 \mathrm{GeV}$ and 10-500 GeV energy ranges LCs.

\begin{tabular}{c|cc|ccc|c}
\hline & \multicolumn{5}{|c}{ Light curve } \\
\cline { 2 - 7 } Parameter & \multicolumn{2}{|c|}{$300 \mathrm{MeV}-3 \mathrm{GeV}$} & \multicolumn{4}{|c|}{$3-10 \mathrm{GeV}$} \\
& Energy Flux & Photon flux & Energy Flux & Photon flux & Energy Flux \\
\hline Angular coeff. & $(1.0 \pm 1.8) \times 10^{-14}$ & $(2.3 \pm 2.4) \times 10^{-17}$ & $(-3.1 \pm 7.6) \times 10^{-15}$ & $(7.3 \pm 7.5) \times 10^{-19}$ & $(-1.2 \pm 1.7) \times 10^{-14}$ & $(-6.4 \pm 4.2) \times 10^{-1}$ \\
Linear coeff. & $(1.13 \pm 0.92) \times 10^{-4}$ & $(1.29 \pm 0.10) \times 10^{-7}$ & $(4.15 \pm 0.33) \times 10^{-5}$ & $(8.90 \pm 0.61) \times 10^{-9}$ & $(2.47 \pm 0.56) \times 10^{-5}$ & $(1.57 \pm 0.20) \times 10^{-9}$ \\
\hline
\end{tabular}

We did not test if the fluxes distributions are also compatible with a Gaussian random process because of the small number of time bins in these LCs and the consequent lack of statistics.

As reported in Section 3.2, the emission centroids in different energy ranges are displaced from Sgr A*'s position in reverse order of energy (see Figures 3.2 and 3.3.). The main reason to create the LCs in these 3 energy bands was to search for "echoes" in the gamma-ray flux. They would be identified as variability in a higher energy LC being observed in a lower energy one after a period of time associated to the light crossing time 
correspondent to the distance between their respective centroids. Since we found no signal of variability in the LCS, the possibility of echoes is unlikely. We further explain and discuss this hypothesis in Section 4.2 .

\subsection{SED}

In Figure 3.19 we show the resulting SED. It was created based on the Universal Model (100 MeV-500 GeV energy range). The spectral model for the source (a log-parabola, Equation 1.2) is shown as a gray line, bounded by the $1 \sigma$ uncertainty. For the energy bins in which 4FGL J1745.6-2859 was detected with $T S>5(\lesssim 2.2 \sigma)$ we show the energy flux $\left(E^{2} d N / d E\right)$ and the $68 \%$ confidence level uncertainties. Otherwise, we show the $95 \%$ confidence level UL.

The best fit model to the data is described as a log-parabola with the parameters $N_{0}$ $=(2.53 \pm 0.04) \times 10^{-12} \mathrm{~cm}^{-2} \mathrm{~s}^{-1} \mathrm{MeV}^{-1}, \alpha=2.59 \pm 0.02, \beta=0.260 \pm 0.010$ and the parameter $E_{0}$ was kept fixed during the analysis in its $4 \mathrm{FGL}$ value (i.e., it was not fit) of $4133 \mathrm{MeV}$.

In section 4.3 we compare this SED with several models for Sgr A* gamma-ray emission. 


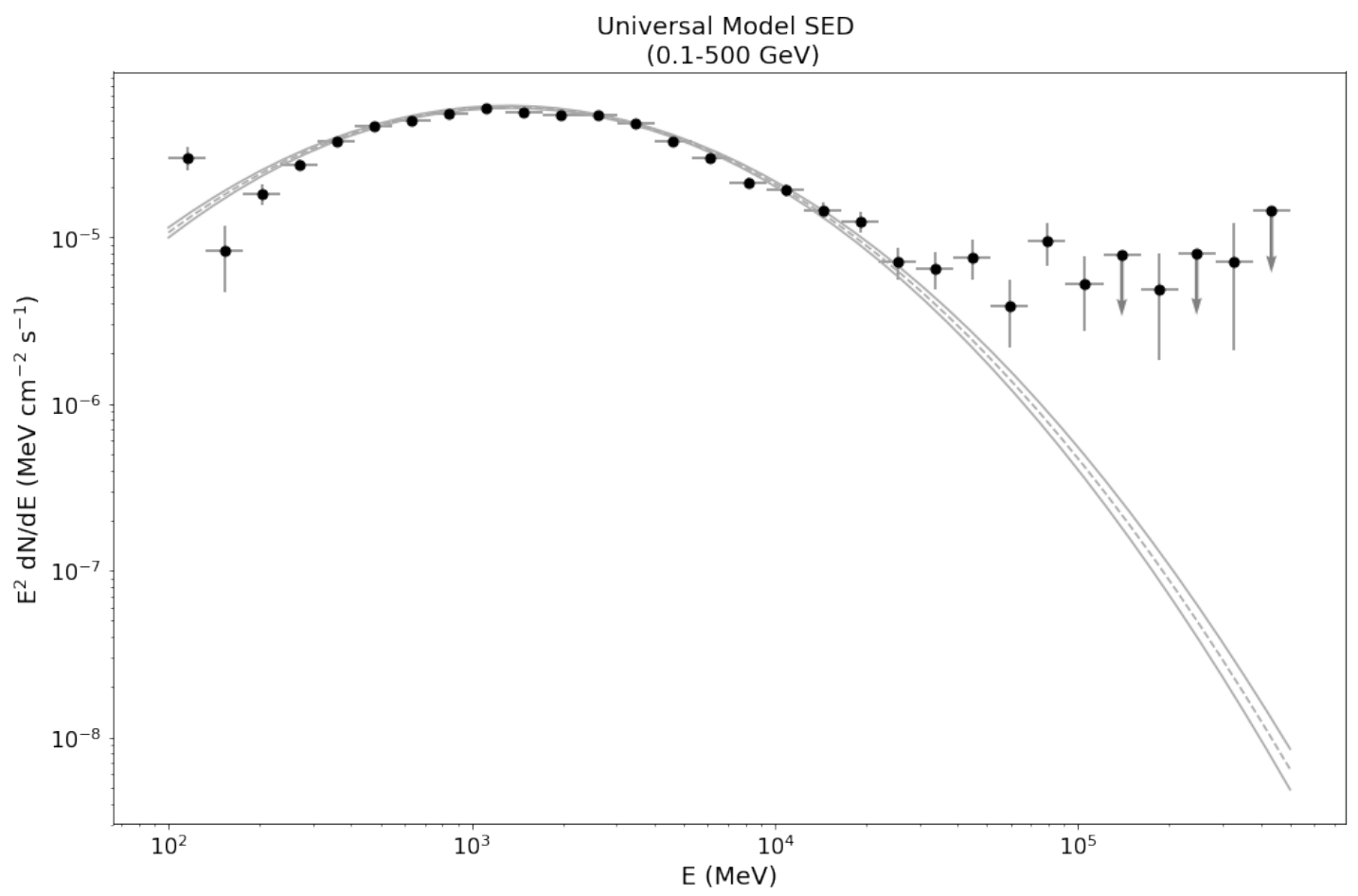

Figure 3.19: The resulting SED for the Universal Model. The dashed gray line is the spectral model for the source (a log-parabola), and the solid lines represent its $1 \sigma$ uncertainty. The error bars represent the $68 \%$ confidence level uncertainties. For points in which the source was detected with significance $\lesssim 2.2 \sigma$, we show the $95 \%$ confidence level UL. 
Chapter 4

\section{Discussion}

Here, we discuss the results presented in Section 3. We start with 4FGL J1745.6-2859's energetics and position, which are compatible with Sgr A*. Then we discuss several candidates for the origin of the observed GC gamma-ray emission, of which Sgr A* is the most likely. Finally, we discuss a variety of models for the variability and SED.

\subsection{Energetics and centroid}

The gamma-ray photons observed with Fermi LAT do not contain any indicator of the distance from our planet at which they were produced. This realization makes it difficult to unequivocally pinpoint the physical nature and location of 4FGL J1745.6-2859 besides noting that its position in the sky approaches Sgr A*'s position as the photon energy increases.

The strong similarity between the electromagnetic energetics of 4FGL J1745.6-2859 and Sgr A*, combined with the positional coincidence, suggests that the gamma-ray point source investigated in this work is associated with the accreting SMBH. Nevertheless, the $0.1^{\circ}-1^{\circ} \mathrm{PSF}$ of Fermi LAT encompasses a region of size $\sim 10-100$ pc around the GC. Even the more constraining limit of $\lesssim 0^{\circ} .24$ for the central source's extension upper limit (Figure 3.4 corresponds to $\lesssim 35 \mathrm{pc}$ at the distance of Sgr A*, thus allowing for several other potential candidates for the gamma-ray production site. Here, we list the most promising ones and, based on the results of our analysis in the four different energy bands and the energetics of the source derived from the Universal Model, we discuss their likelihood at accounting for 4FGL J1745.6-2859. 
Evaluating the source with the Universal Model (100 MeV-500 GeV), we obtain a gamma-ray energy flux of $(3.26 \pm 0.05) \times 10^{-10} \mathrm{erg} \mathrm{cm}^{-2} \mathrm{~s}^{-1}$ (Section 3.1). By assuming a distance of $8.2 \mathrm{kpc}$ for 4FGL J1745.6-2859 (i.e., that it is located at the GC) we obtain the interesting result that its gamma-ray luminosity is similar to Sgr A*'s radio to X-ray luminosity, which is about $10^{36} \mathrm{erg} \mathrm{s}^{-1}$. Taken together with the positional coincidence with Sgr A* (and the other circumstantial evidence arising from the temporal behavior and emissions models compatible with the expected from Sgr A*, see Sections 4.2 and 4.3 it is a compelling suggestion that 4FGL J1745.6-2859 is associated with Sgr A*.

Also, when we use our flux results of the four energy bands (Section 3.1 and Table 3.1) to investigate other competing candidates for this emission, we find that Sgr A* is the most compelling contender (Section 4.1.1). Another information used in the evaluation of these potential candidates is the central source position as a function of energy, presented in Section 3.2 (Figure 3.2).

If we consider only the three highest-energy ranges used in this analysis, the centroid emission moves in the direction of Sgr A* as the photon energy is increased (Figures 3.2 and 3.3. Assuming a distance of $8.2 \mathrm{kpc}$, the projected distances to Sgr A* as a function of energy varies from $3.6 \pm 1.3 \mathrm{pc}(300 \mathrm{MeV}-3 \mathrm{GeV})$ to $0.4 \pm 1.4 \mathrm{pc}(10-500 \mathrm{GeV})$ (Figure 3.3). This suggests that the particle populations responsible for the gamma-rays detected in the three bands are accelerated by the same process, originating in the surroundings of Sgr A*. The location centroid for the higher and the lower-energy bands are consistent within $1 \sigma$ with Sgr A*'s.

As discussed in Section 1.5.3, the impact of source confusion on Fermi-LAT observations is greater at lower energies due to the PSF broadening. The localization uncertainties are considerable larger at low energies, as well as the limit on the angular extension, which creates the possibility that several other sources and processes are contributing to the lower energy flux.

In Figure 4.1 we show that the emission centroids are compatible with the gas distribution in the CND and mini-spiral in the inner parsecs of the GC. This coincidence may indicate that the gamma-ray arising from the GC is due to hadronic processes (Section 1.6.2). In this case, protons accelerated by (or very nearby) Sgr A* interact with the gas content in the CND generating pions and, consequently, gamma-ray photons. Scenarios in which the gamma-ray emission from the GC has a hadronic origin are further described 
below.

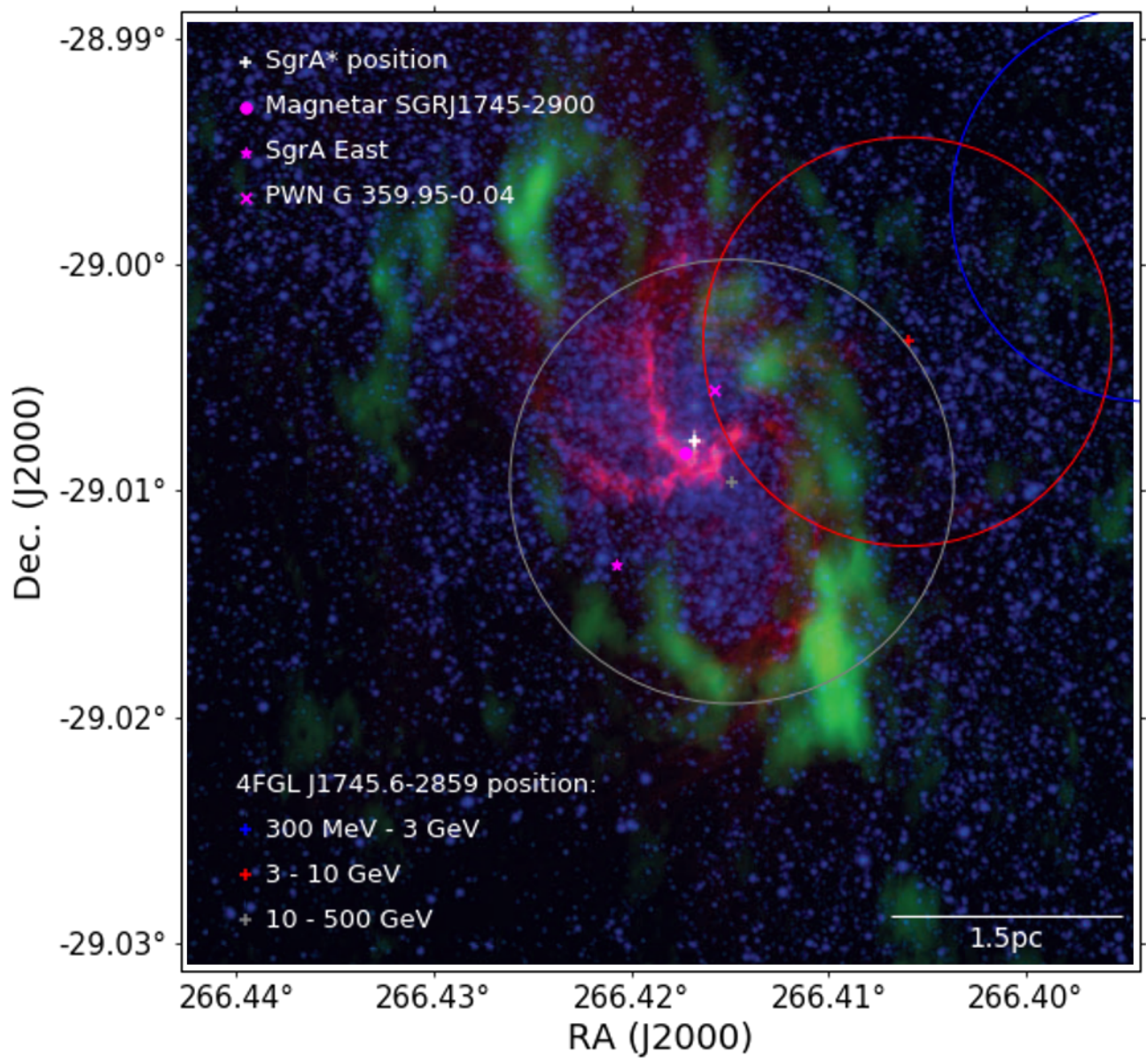

Figure 4.1: In this plot, we combine the position of the central source as a function of energy with a background showing the gas distribution in the CND and mini-spiral (adapted from Genzel et al. 2010). The circles follow the notation introduced in Figure 3.2 and the colors of the background are the same as in Figure 1.1 .

\subsubsection{Nature of emission}

Other than Sgr A*, there are several other potential candidates for the gamma-ray production site. Here, we list the most promising ones and discuss whether which candidate model can successfully explain 4FGL J1745.6-2859.

The SMBH: Aharonian and Neronov (2005a) argue that due to Sgr A*'s low bolometric luminosity compared to other SBMHs, the gamma-rays produced at small radii can escape 
the source and be detected by Fermi LAT because the absorption through photon-photon pair production should be unimportant. Aharonian and Neronov (2005a) considered three scenarios for the TeV photons detected by H.E.S.S., two being hadronic and one leptonic. The first hadronic model considers emission related to accelerated protons producing gamma-rays through synchrotron and curvature radiation. It predicts energy flux lower than a few $10^{-12} \mathrm{erg}^{-2} \mathrm{~s}^{-1}$ in the energy range studied in this work, below the values we observed. The second hadronic scenario considers lower energy protons accelerated by the electric field close to the event horizon or by shocks in the accretion disks. Some parametrizations of this model predict very peaked SEDs in the energy ranges used in this work. Since these SEDs are very narrow, the energy fluxes they predict are consistent only with the observations in one of the four energy ranges we used in this work. Their leptonic model, in its turn, also fails to explain Fermi-LAT's observation of 4FGL J1745.6-2859:

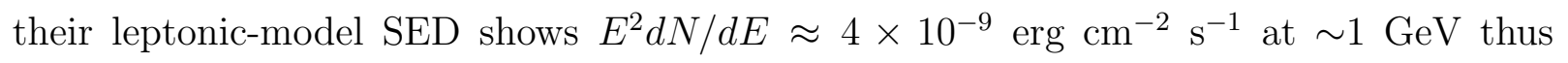
overpredicting our observed energy flux at this energy by $\sim 2$ orders of magnitude.

A “plerion" in Sgr A*'s winds: Atoyan and Dermer (2004) propose a model for Sgr A* in which the quiescent radio and the flaring NIR and X-ray emission are generated by synchrotron radiation from the RIAF. The wind from the RIAF, in a process similar to pulsar-powered plerions 1 , generates the quiescent X-ray and $\mathrm{TeV}$ emission at the wind termination shock at a distance of about $3 \times 10^{16} \mathrm{~cm}\left(\sim 25,000 R_{S}\right)$ from the SMBH. Although it can explain H.E.S.S.' TeV observations, their model is not sufficient to explain the MeV-GeV reported in this work. Even if we consider Sagittarius A West bremsstrahlung emission and the emission from a larger plerion (inflated to pc scales), which are prominent in the energy range used in this work (e.g., their Figure 1), the energy flux we detected is still about one order of magnitude higher. On the other hand, Kusunose and Takahara (2012) used 25 months of Fermi LAT's data for the GC (reported by Chernyakova et al. 2011) and proposed a similar leptonic model in which electrons escaping from the vicinity of $\operatorname{Sgr} \mathrm{A} *$ accumulate in a region with a size of $10^{18} \mathrm{~cm}$ where the gammarays are produced by IC scattering of soft photons emitted by stars and dust around the

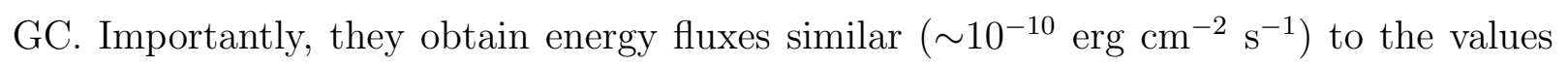
observed here.

\footnotetext{
${ }^{1}$ A plerion is a supernova remnant that, instead of being a shell, has it center is "filled" by energetic particles streaming from a rotating pulsar. The Crab Nebula is the archetypal plerion.
} 
The interaction between the dense molecular clouds with cosmic rays: As an explanation for the gamma-ray emission from the GC, Aharonian and Neronov (2005b) presented a model of proton-proton interactions between the protons accelerated near the SMBH and the dense gas in the central $10 \mathrm{pc}$ of the Galaxy which are followed by $\pi^{0}$ decay to gamma-rays. Aharonian \& Neronov's work was published before the beginning of operations of Fermi LAT. Their results are inconsistent with our observations, predicting energy fluxes $\sim 1$ order of magnitude lower than our observations.

More recent models take into account Fermi-LAT observations. For instance, Chernyakova et al. (2011) use the first 25 months of Fermi-LAT and H.E.S.S. data to create a hadronic model in which relativistic protons (presumably accelerated near Sgr A*) interact with the gas in the inner parsecs of the Galaxy. Linden et al. (2012) developed a similar model. Another hadronic model is proposed by Fatuzzo and Melia (2012) where they consider a two-phase environment surrounding Sgr A*: an inner high-density "torus" and the surrounding interstellar medium filled with shocked stellar winds which they call the "wind zone". Fermi gamma-rays would be produced in the "torus" and the higher energies would come mostly from the "wind zone". Guo et al. (2013) propose a hybrid model. In their scenario, protons and electrons are accelerated in the GC (possibly around Sgr A*). Collisions between the protons and the interstellar gas would produce the $\mathrm{TeV}$ gamma-rays and the electrons would IC scatter the soft background photons.

The four "Fermi-era" models mentioned above - as reported by Chernyakova et al. (2011); Linden et al. (2012); Fatuzzo and Melia (2012); Guo et al. (2013) — are consistent with our observations, except in the lower energy band we used. This energy band is the one most subject to source confusion and to the impacts of the Galactic diffuse emission. This could explain why we observe greater energy fluxes in the 60-300 MeV energy band than the prediction of these models: in addition to the gamma-rays created by the interaction between cosmic rays originated by (or nearby) Sgr A*, there is also a contribution from other sources. When Ahnen, M. L. et al. (2017) compared Fermi LAT's data reported by Malyshev et al. (2015) with the "Fermi-era" models, the lower energy data $(\lesssim 200 \mathrm{MeV})$ also showed greater fluxes than the models' predictions.

The PWN G 359.95-0.04: This X-ray nebula was discovered by Wang et al. (2006) with a projected distance of only $0.32 \mathrm{pc}$ from $\mathrm{Sgr} \mathrm{A} *$ and was proposed as an explanation for the $\mathrm{TeV}$ emission observed in the GC. Hinton and Aharonian (2007) constructed theoretical 
SEDs for this source based on Chandra X-Ray Observatory's detection and supposing that the TeV emission of the H.E.S.S. source HESS J1745-290 is from the PWN. Their models (e.g., their Figure 4) predicts energy fluxes on the order of $\sim 10^{-12} \mathrm{erg} \mathrm{cm}^{-2} \mathrm{~s}^{-1}$ for the energy ranges studied in this work. This is more than one order of magnitude lower than the energy fluxes we measured for 4FGL J1745.6-2859. This indicates that G 359.95-0.04 is not a good candidate to explain 4FGL J1745.6-2859's emission.

The supernova remnant Sagittarius A East: Sagittarius A East is a SNR located in the inner parsecs of the Galaxy. Crocker et al. (2005) proposed it as the source of the TeV $\gamma$-rays from the GC. On the other hand, Aharonian et al. (2009) and Acero et al. (2010) ruled out this association based on H.E.S.S. observations that show the origin of the $\mathrm{TeV}$ emission, although still coincident with Sagittarius A East's extended radio shell, coming from a region where the radio flux is comparatively low and significantly displaced from the radio maximum, as indicated in Figure 3.2. The same argument can be used to rule out a physical link between the SNR and the 4FGL J1745.6-2859 in energies above 300 $\mathrm{MeV}$.

The magnetar SGRJ 1745-2900: This object was first detected during a flare in 2013 with Swift's X-Ray Telescope (Kennea et al., 2013) and NuSTAR (Mori et al., 2013). 4FGL J1745.6-2859's gamma-ray light curve shows no sign of variability during this period (Section 3.3). Also, it is predicted that the high-energy portion of the spectra of magnetars peak at a few $\mathrm{MeV}$ (Thompson and Beloborodov, 2005), while our work clearly shows emission from 4FGL J1745.6-2859 at energies > $10 \mathrm{GeV}$.

Self-annihilating dark matter particles accumulating at the GC: Self-annihilating dark matter particles could explain the GC gamma-ray excess (Hooper and Goodenough, 2011; Hooper and Linden, 2011), a surplus of $\sim \mathrm{GeV}$ diffuse emission that cannot be explained by the known cataloged sources (and see Di Mauro 2021 for a recent analysis of the GC excess). But is not a satisfactory explanation for 4FGL J1745.6-2859 since this is a point-like source rather than spatially extended, as expected for dark matter emission.

A population of pulsars surrounding the GC: The gamma-ray spectra of pulsars and millisecond pulsars can be described as a power-law with an exponential cutoff above a few GeV (Abdo et al. 2010). de Menezes et al. (2019) studied the gamma-ray emission of globular clusters in the Milky Way - attributed to their large population of millisecond pulsars - and found no significant flux above $\sim 10 \mathrm{GeV}$. In contrast with that, the point 
source 4FGL J1745.6-2859 is detected at energies above that as indicated in Table 3 and Figure 2.8. Its hard spectrum is also not consistent with a pulsar (or population) in the line of sight.

A background blazar fortuitously aligned with Sgr A*: From the $\gtrsim 5000$ sources in the 4FGL Catalog, 4000 are identified or associated with plausible counterparts observed at other wavelengths. More than $80 \%$ of these are blazars (The Fermi-LAT Collaboration, 2019). Here we consider the possibility of a background blazar, fortuitously aligned with Sgr A*, as the explanation for the central gamma-ray source. We should stress that the lack of evidence of this putative object in other wavelengths is already an indication that this is not a likely supposition. Here we consider the energetics obtained with the Universal Model (100 MeV-500 GeV) to assess if this is a satisfactory hypothesis. The average energy flux of the 4FGL blazars in the $100 \mathrm{MeV}-100 \mathrm{GeV}$ energy range is $(8.989 \pm$ $0.014) \times 10^{-12} \mathrm{erg} \mathrm{cm}^{-2} \mathrm{~s}^{-1}$. The energy flux we measured for the central source is $(3.26 \pm$ $0.05) \times 10^{-10} \mathrm{erg} \mathrm{cm}^{-2} \mathrm{~s}^{-1}$ (in the slightly larger energy band of $100 \mathrm{MeV}-500 \mathrm{GeV}$ ). This would put the hypothesized source among the $0.2 \%$ brightest blazars in 4FGL (the $8^{\text {th }}$ brightest in a list of $\gtrsim 3400$, see Figure 4.2). The fact that this bright blazar was never observed in other wavelengths (in particular radio) already rules out this possibility. The combination of three very unlikely events - i.e. an atypically bright gamma-ray blazar, that is also unusually dim (so far undetected) in other wavelengths and is perfectly aligned with the Galactic Center - would have to be acting in concert to explain the central source as a blazar. Another reason for discarding the blazar hypothesis is that we don't see "blazar-like" variability from the GC (see Section 4.2). So we can safely rule out this possibility.

The central cluster of massive stars: A young and dense stellar cluster lies in the GC (Do et al., 2013). Quataert and Loeb (2005) propose a model in which shocks from stellar winds can efficiently accelerate electrons to relativistic energies. Then, they IC scatter the ambient radiation field producing gamma-rays from $\sim \mathrm{GeV}$ to $\sim 10 \mathrm{TeV}$. Considering a distance of $8.2 \mathrm{kpc}$ to the GC, their model predicts energy flux one order of magnitude lower than our observations. Aharonian et al. (2019) have discussed young stellar clusters as sources of cosmic rays. They suggest that the cosmic rays responsible for the diffuse very high energy gamma-ray form the GC are accelerated by the local stellar clusters. This emission, though, is extended while we did not find evidence of extension for 4FGL 
J1745.6-2859.

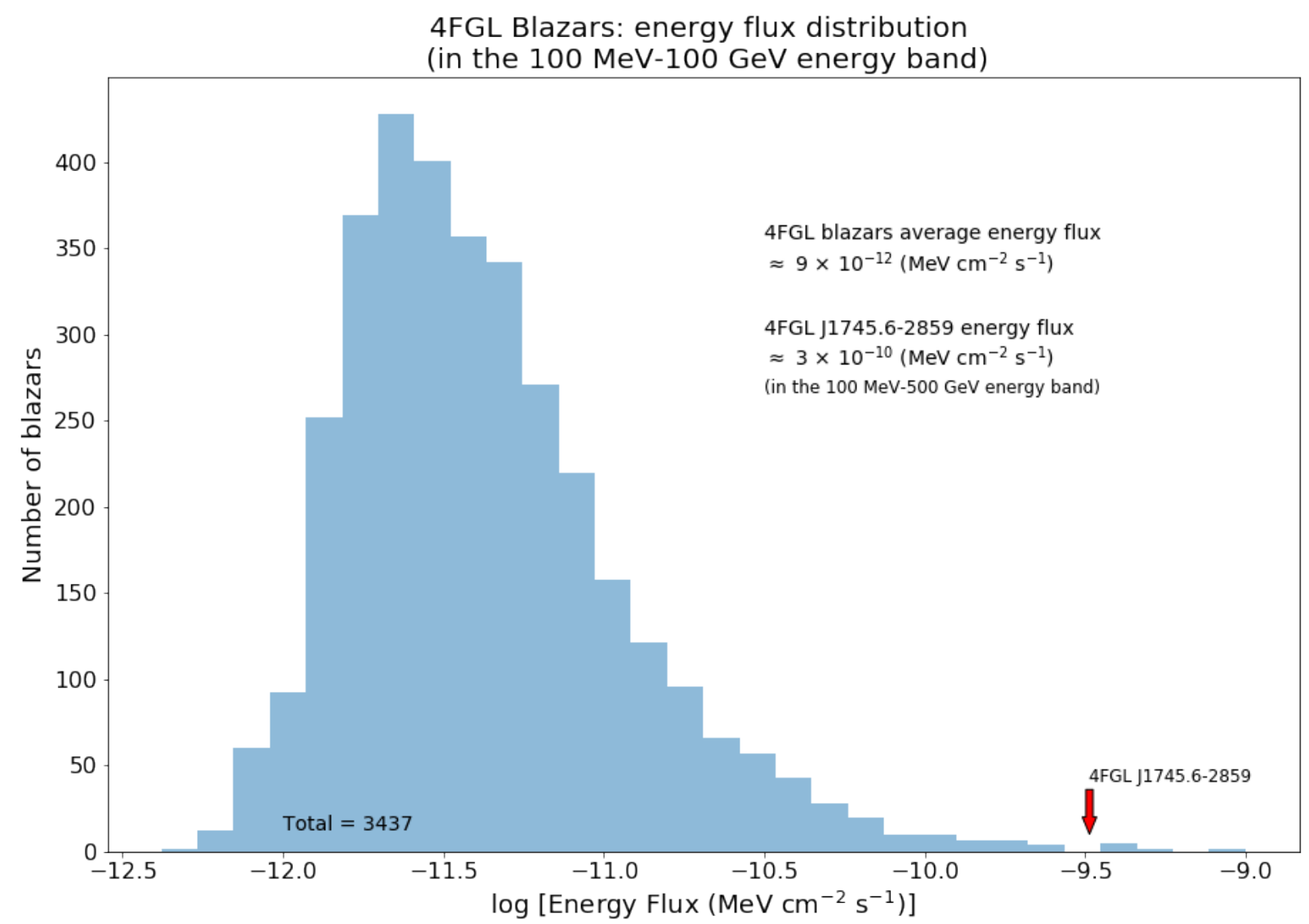

Figure 4.2: The energy flux distribution of the 3447 4FGL sources associated with blazars in the 100 MeV-100 GeV energy band. The GC source, 4FGL J1745.6-2859, energy flux is consistent with the top the $0.2 \%$ brightest blazars in the Catalog.

Except for the models constructed around Fermi LAT's data (the "Fermi-era" models), most of the candidates listed above are unlikely to be solely responsible (if responsible at all) for 4FGL J1745.6-2859's emission. It is possible that some of them could explain, individually or together, the lower energy emission where Fermi LAT's PSF is broadest. One way to separate the contributions of different candidates at lower energies is through modeling the MeV-to-GeV spectral energy distribution of the point source. This is discussed in Section 4.3.

In Sections 4.2 and 4.3 we further discuss the "Fermi-era" models in view of 4FGL J1745.6-2859's temporal variability and SED. 


\subsection{On the temporal flux evolution}

Here, we discuss the time evolution of the 4FGL J1745.6-2859's gamma-ray flux. We start this Section discussing the 15 days bins LC. After that, we analyze the LCs created for the models with tighter energy bands generated from the Universal model.

As stated in Table 2.1, the systematic uncertainties in our LCs are small, representing $\lesssim 10 \%$ of the error bars' sizes for every energy range used in this work (except for the 100$500 \mathrm{GeV}$ energy band in which it is $<20 \%$ ). For this reason, their impact in the results are neglected in our discussion.

\subsubsection{The 15 days bins $L C$}

The 15 days bins flux distribution is compatible with a Gaussian, which indicates that it arises from a random process (see Tables 3.2 and 3.3). This, alone, does not exclude the possibility that the source, although in a steady state most of the time, has very short periods of variability. The results presented in both panels of Figure 3.7, though, indicate that this is not the case. Only very few data points incompatible within $2 \sigma$ with the 5 bins rolling average flux. If the source is flaring in gamma-rays, the 15 days time resolution used in our work, close to the limit allowed by Fermi-LAT, is not enough to detect it.

The "Fermi-era" models try to explain the Fermi GC source as the manifestation of Sgr $A *$. Most of them were created taking into account the temporal behavior. We discuss them in view of our updated LCs.

Chernyakova et al. (2011) work was based on the Fermi-LAT source 1FGL J1745.6-2900 (See Table 1.2 for a list of the GC source in the different Fermi-LAT catalogs), together with H.E.S.S. observations, to create a hadronic model in which relativistic protons (presumably accelerated near Sgr $A *$ ) interact with the gas in the inner parsecs of the Galaxy. Their analysis of the Fermi data did not uncover any statistically significant variability of 1FGL J1745.6-2900 at GeV energies on the month timescale. This is compatible with our findings.

Fatuzzo and Melia (2012) proposes another hadronic model, described in Section 4.1.1. They suggest that the Fermi-LAT and H.E.S.S. data they used do not provide evidence for strong gamma-ray variability in Sgr A*, which is compatible with our updated and more detailed observations. 
Since electrons have much shorter cooling time than protons, the use of leptonic models to explain a steady source is challenging. Kusunose and Takahara (2012) propose a leptonic model in which relativistic electrons supplied by flare events that occur near Sgr A* accumulate in a region with a size of $10^{18} \mathrm{~cm}$. There the gamma-rays are produced by IC scattering of soft photons emitted by stars and dust around the GC. The parameters they use for the magnetic field and soft photon energy in the GC result in the electron cooling time being longer than the escape time which justifies their steady state homogeneous model. Also, since the flares intervals that accelerate the electrons are of only a few hours and much shorter than the dynamical timescale of the emission, they assume a continuous injection of electrons in their model.

Our results are also compatible with Malyshev et al. (2015) observations of the GC source 2FGL J1745.6-2858. This Fermi-LAT source was split into two different sources in the following version of the Catalog: 3FGL J1745.6-2859c and 3FGL J1745.3-2903c. They found no temporal variability on yearlong timescales in the 1-10 GeV energy range they used. This energy band is not exactly the same as any used in our work. But we have LCs in the $300 \mathrm{MeV}-3 \mathrm{GeV}$ and 3-10 GeV energy bands and both are also consistent with constant flux (i.e., can be fitted with a linear function with angular coefficient compatible with zero, see Table 3.5). Our work has the advantages of having shorter time bins (90 days versus 1 year), of using more data (11.3 versus 6 years) and of employing a more recent version of the Fermi Catalogs (4FGL versus 2FGL).

Malyshev et al. (2015) proposed a leptonic model for the GC source emission. The combination of the emission from electrons injected during a strong Sgr A*'s flare occurred between $\sim 100$ to 400 years ago (see Section 1.3.3.4) with emission from much weaker, recent activity. They propose ionisation, bremsstrahlung, IC, and synchrotron mechanisms as the main energy-loss channels. Their observations cover only the $\lesssim 6.2$ years of Fermi operation. For longer timescales, they made a testable prediction of a $5-10 \%$ decrease in the $1-10 \mathrm{GeV}$ flux. Our $\sim 11.3$ years observations are not compatible with this prediction, since 4FGL J1745.6-2859 flux is constant either in the energy bands that cover Malyshev et al. (2015) LC and also in the whole energy band used in the Universal Model (100 $\mathrm{MeV}-500 \mathrm{GeV})$.

The steady emission is a characteristic of the gamma-ray flux from the GC. In addition to the Fermi-LAT data reported discussed above, the same is true for the IACT and EAS 
observations. No variability has been detected yet in Fermi-LAT energy levels and above (Aharonian et al., 2009; Chernyakova et al., 2011; Malyshev et al., 2015; Ahnen, M. L. et al., 2017; H.E.S.S. Collaboration et al., 2018).

The flares from Sgr A* in longer wavelengths are frequent. They are typically daily in X-rays and even more frequent in the NIR. Also, they have short duration, lasting usually for tens of minutes in X-rays and NIR. See Section 1.3.3.3 for a description of the characteristics of the flares. If the same processes that generate them are also generating gamma-ray flares with similar rate and duration, these flares could not be identified with the 15 days bins LCs we created for this thesis. Unfortunately, smaller time bins lead to poor statistics because of the small number of counts and also the increased systematic uncertainties. At the same time that our results are compatible with a steady source in 15 days timescales, they do not rule out the possibility of variability on shorter timescales.

With this caveat in mind, the lack of coordinated variability in high and low energies arising from Sgr A* may indicate that the gamma-ray emission mechanism differs substantially from the low-energy regime. Models by which the longer wavelenghts emission are generated very close to Sgr A* while the gamma-rays are produced farther away by highenergy protons interacting with the interstellar medium could explain our observations. This framework was originally proposed by Aharonian and Neronov (2005b) and explored in several models thereafter (e.g., Chernyakova et al. 2011; Linden et al. 2012; Fatuzzo and

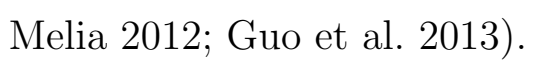

Here, we list a few examples of remarkable events that could enhance the gamma-ray emission from the GC or flares in different wavelenghts originated by Sgr A* during the period covered by our LCs. There is no notable variability in the $100 \mathrm{MeV}-500 \mathrm{GeV}$ flux, as shown in Figures 4.4 and 4.5 , in which we identify the following events:

1. The multiwavelength flare from April $1^{\text {st }} 2009$ : Trap et al. (2011) reports concurrent X-ray, NIR, sub-mm, and gamma-ray (assuming 1FGL J1745.6-2900 is associated with the SMBH) observations of Sgr A*. In April $1^{\text {st }} 2009$ they detected a NIR flare (lasting for $\sim 4.5 \mathrm{ks})$ followed $(\sim 12 \mathrm{ks}$ after $)$ by a sub-mm flare among the brightest ever detected (lasting for $\sim 9 \mathrm{ks}$ ). They detected no significant peak in the $\mathrm{GeV}$ energy range, a result compatible with our LCs. Their LCs are show in Figure 4.3.

2. X-ray flare from February $9^{\text {th }}$ 2012: Nowak et al. (2012) reported the brightest, at 


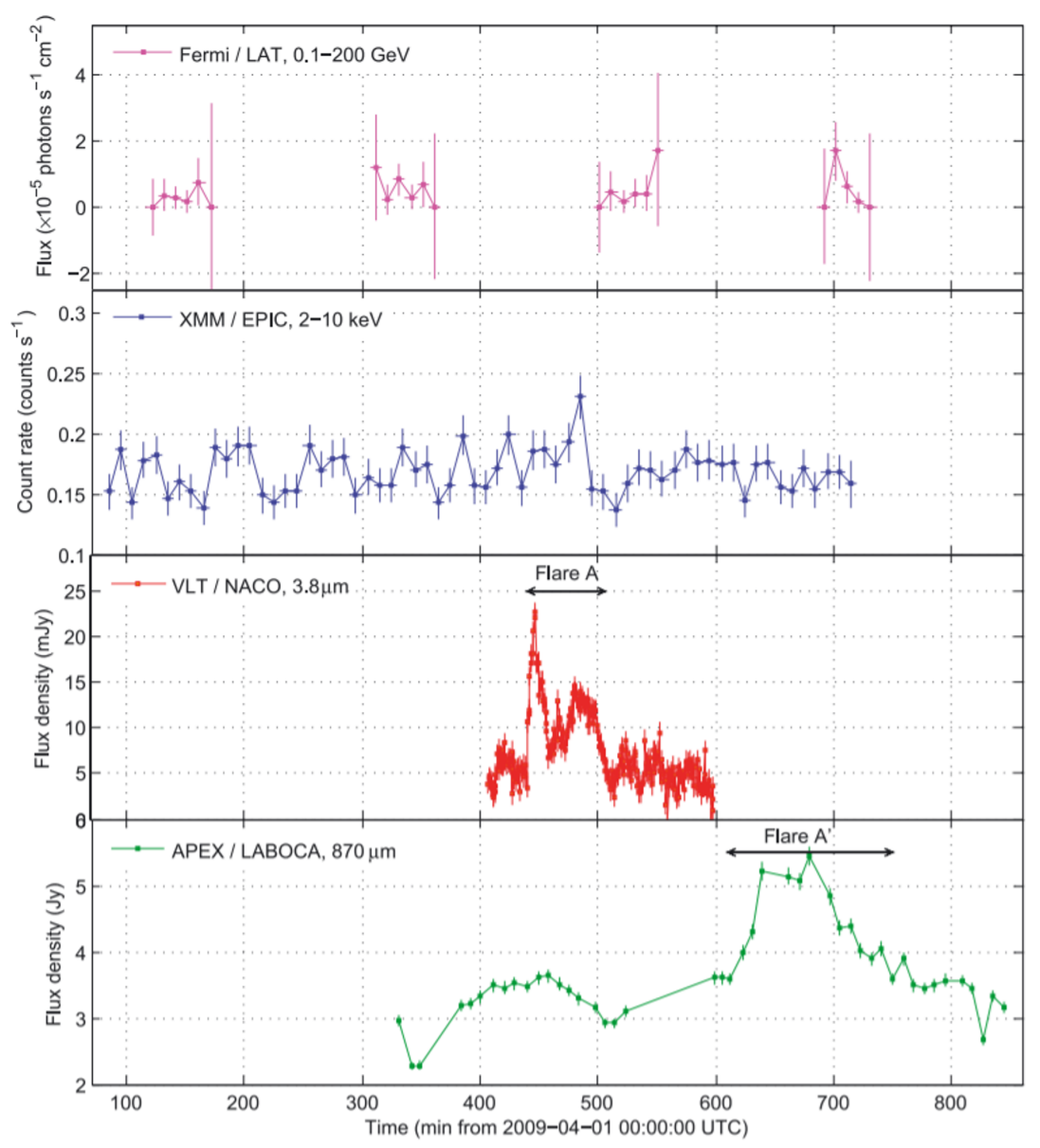

Figure 4.3: Trap et al. (2011) observations of Sgr A* from April $1^{\text {st }}$ 2009. The upper panel corresponds to Fermi-LAT's monitoring in the $100 \mathrm{MeV}-200 \mathrm{GeV}$ energy range. The next panel shows XMM-Newton observations in energies between 2-10 keV. The third panel shows, in red, the NIR flare detected by the VLT in the $3.8 \mu \mathrm{m}$ wavelenght. A flare was also observed in the $870 \mu \mathrm{m}$ by the Atacama Pathfinder Experiment $\sim 12 \mathrm{ks}$ later as shown in the bottom panel. Source: Trap et al. (2011).

the time, X-ray flare from Sgr A*, observed on February $9^{\text {th }} 2012$ with Chandra X-Ray Observatory. The flux rose to more than $100 \times$ quiescence and lasted for $\sim 5.6$ ks. This flare is depicted in the left panel of Figure 1.7. The total energy released in the $2-10 \mathrm{keV}$ energy band was estimated to be $\sim 10^{39}$ erg. Guo et al. (2013) made an order of magnitude estimate of the gamma-ray emission expected from this event supposing that the total energy of this accretion event is about four orders of magnitude higher than the X-ray emission, $\sim 10^{43}$ erg (Mezger et al., 1996), and that $\sim 10 \%$ of it converted to acceleration of cosmic rays and the framework of their "Fermi-era" model. The total gamma-ray flux expected was too low to be detectable by the current astrophysical gamma-ray observatories. 
3. X-ray flare from September $14^{\text {th }}$ 2013: Reported by Haggard et al. (2019), this is the brightest Chandra X-Ray flare reported so far, reaching more than $600 \times$ the quiescent X-ray emission. The flare lasted for $5.7 \mathrm{ks}$, with a rapid rise time of $1.5 \mathrm{ks}$ and a decay time of $2.5 \mathrm{ks}$.

4. The pericenter of the G2 object orbit around Sgr A* on March $7^{\text {th }}$ 2014: Plewa et al. (2017) reported the post pericenter $\left(\right.$ at $\left.\approx 1560 \mathrm{R}_{S}\right)$ evolution of the G2 object (more details in Section 1.3.2 emission. They find no evidence of a drag force or any strong hydrodynamic interaction with the hot gas in the inner accretion zone.

5. The multiwavelength flare from June $18^{\text {th }}$ 2014: This event, reported by Fazio et al. (2018) provides the first evidence of coeval structure between NIR and flux increase. It was composed by a double-peaked NIR flare that lasted for $\lesssim 4 \mathrm{ks}$ and a flare with much longer duration of $\lesssim 20 \mathrm{ks}$. There was no good quality observations in X-rays, so it is not known whether the flare produced any emission at these frequencies. The authors could not rule out that the unusual simultaneous flares was just a coincidence.

6. X-ray flare from October $20^{t h}$ 2014: Also reported by Haggard et al. (2019), this flare peak luminosity was of more than $245 \times$ the quiescent X-ray emission and lasted for $3.4 \mathrm{ks}$.

7. The multiwavelength flare from May $14^{\text {th }}$ 2015: This is the first example of the sequence of , X-ray and NIR increase in Sgr A*'s flux, all occurring within $\sim 1 \mathrm{hr}$ (Fazio et al., 2018). In this unusual event, a single-peaked flare was coincident at X-ray ( $\sim 500$ s duration) and $(\sim 2 \mathrm{ks})$ wavelengths, but the NIR peak was delayed by $\sim 4 \mathrm{ks}$ (and lasted for $\sim 2 \mathrm{ks}$ ). This flare is unprecedented in several aspects: the peak precedes the X-ray peak by $\sim 1.6 \mathrm{ks}$, and the NIR peak is delayed from the peak of the emission. This has never been observed in previous events. The authors warn that observation time before and after the flare is very limited, and it is possible that the peaks at different wavelengths may be associated with other flares that either preceded or followed the observed flare.

8. The pericenter of the S2 star orbit around Sgr A* on May 19 $9^{\text {th }}$ 2018: GRAVITY Collaboration et al. (2020) report the first detection of the General Relativity Schwarzschild Precession in S2's orbit close to the pericenter (at $\approx 1400 \mathrm{R}_{S}$ ) of its orbit. The 
close encounter could provide a surplus of accreting material to the SMBH over the following few years by providing fuel due to the mass-loss from the star.

9. The unprecedented NIR flare of May, $13^{\text {th }}$ 2019: Do et al. (2019) observed the brightest NIR ever recorded from Sgr A*. They observed a factor of 75 change in the NIR flux over a 2-hour time span. The maximum occurred at the beginning of the observations, suggesting that Sgr A* was likely even brighter earlier in the night. The authors suggested that the flare was a consequence of an increase in the SMBH mass accretion rate. On the other hand, we propose a model in which the flare was the result of particle acceleration to nonthermal energies, leading to an explosive event in the innermost parts of the accretion flow (Gutiérrez et al., 2020).

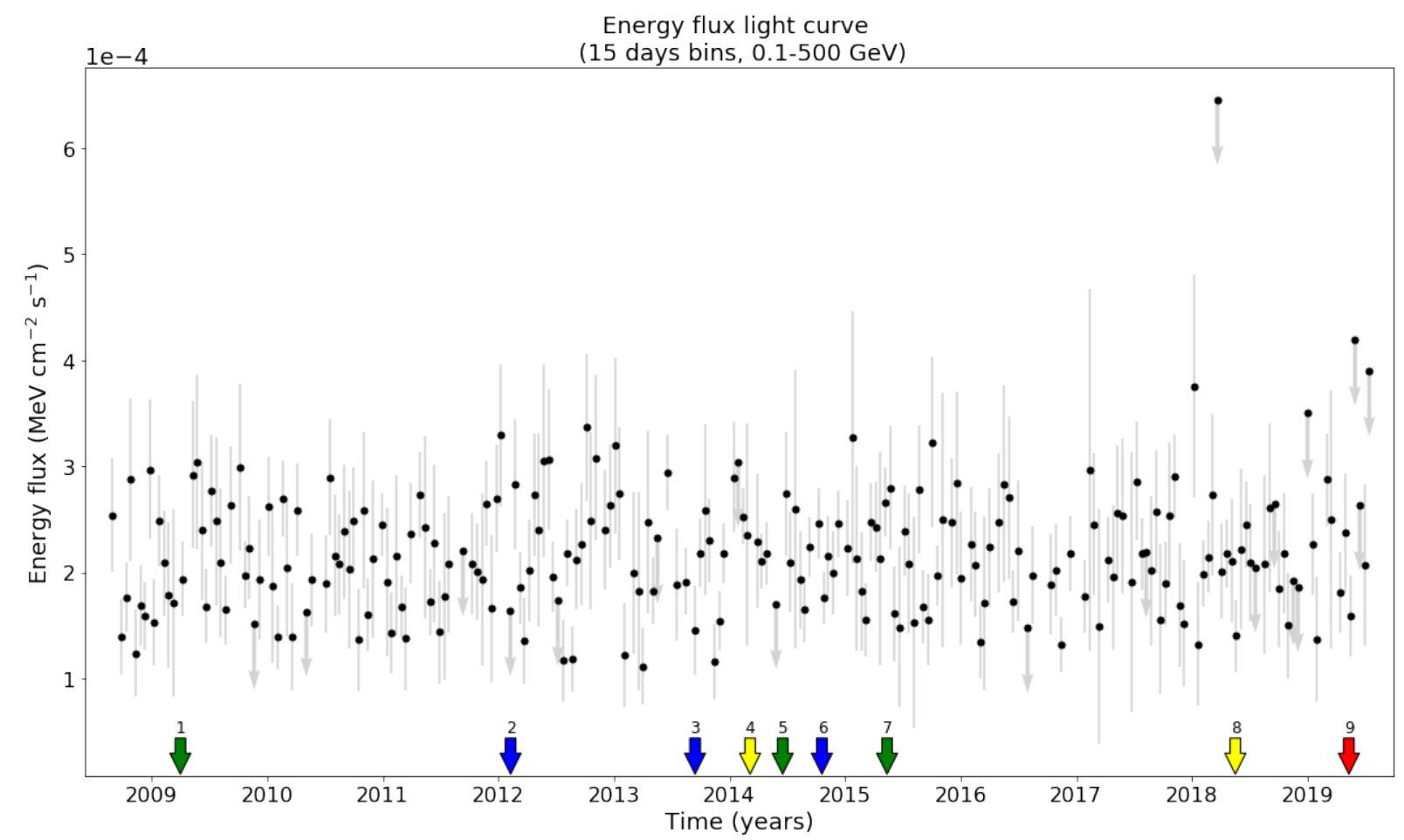

Figure 4.4: 15 days energy flux LC of 4FGL J1745.6-2859 in the $100 \mathrm{MeV}-500 \mathrm{GeV}$ energy band. This is the same as the upper panel of Figure 2.12, with the inclusion of several arrows at the bottom of the plot, indicating remarkable events associated to Sgr A*: multiwavelength flare (green), X-ray flares (blue), orbital ephemeris (yellow) and NIR flare (red). The number above the arrows relate to the events listed in Section 4.2.1

Models for the emission above a few $\mathrm{MeV}$ from the GC must take into account that this emission shows no temporal variability since the first observations. Three of the "Fermiera" models were created in this context, two hadronic (Chernyakova et al., 2011; Fatuzzo 


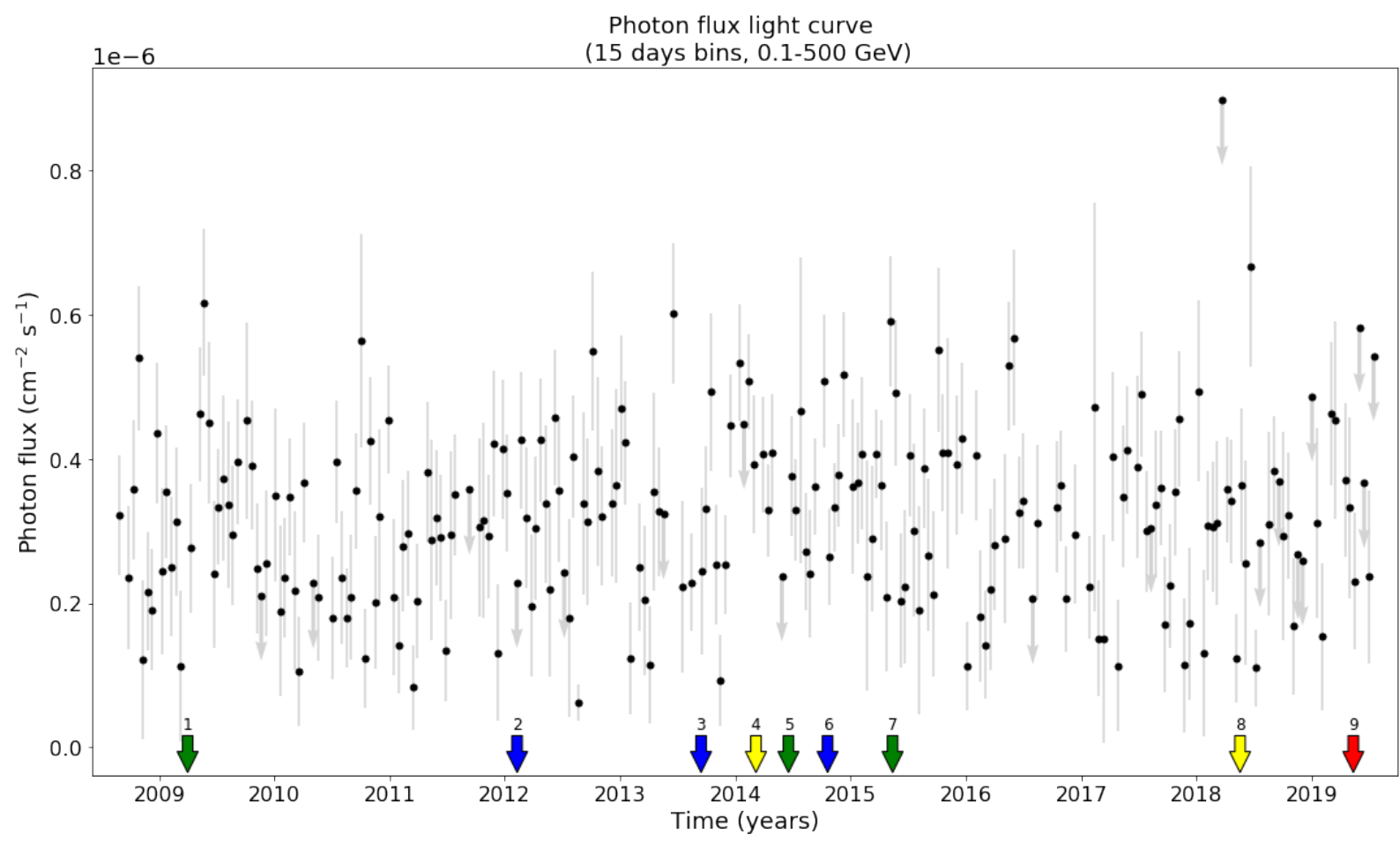

Figure 4.5: 15 days photon flux LC of 4FGL J1745.6-2859 in the $100 \mathrm{MeV}-500 \mathrm{GeV}$ energy band. This is identical to the LC shown in he bottom panel of Figure 2.12 with the addition of the same arrows described in Figure 4.4

and Melia, 2012) and one leptonic (Kusunose and Takahara, 2012). This shows that both type of models can explain the lack of variability in the GC gamma-ray emission.

In this work, we also tried to incorporate the source imaging into this analysis. In the next Section, we describe our search of "echoes" in the LCs associated with different energy bands, whose centroids are displaced from Sgr A*'s position in inverse order of photon energy (Section 3.2.

\subsubsection{The LCs created for the narrower energy bands}

Here we discuss the LCs created for the Models in the narrower energy bands: 300 MeV-3 GeV, 3-10 GeV and 10-500 GeV. We already showed that the emission centroids in these bands are displaced from each other (see Figure 3.2).

Either for the leptonic and the hadronic astrophysical gamma-ray scenarios (Section 1.6) the cross sections governing the electromagnetic emission increase with the cosmic ray kinetic energy (Compton 1923; Kafexhiu et al. 2014, respectively). This could explain why the point source's positions depart from $\mathrm{Sgr} A *$ in the lower energies (considering only the models above $300 \mathrm{MeV}$ ). If that is the case, we would expect the most energetic cosmic rays 
accelerated by Sgr A* to interact first with photons (if leptonic) or gas (if hadronic) closer to their origin. A hypothetical fluctuation on the rate of energetic cosmic rays generated by Sgr A* would appear as "echoes" in the LCs with the variation manifesting first in higher energies and later, after the light crossing time between the centroids, in lower energies. Figure 4.6 shows a cartoonistic version of this simple model, considering the distances of the emission centroids exhibited in Figures 3.2 and 3.3 as examples. Although very naive, this model is easy to investigate since we have the LCs and the source's centroid into three different energy bands.
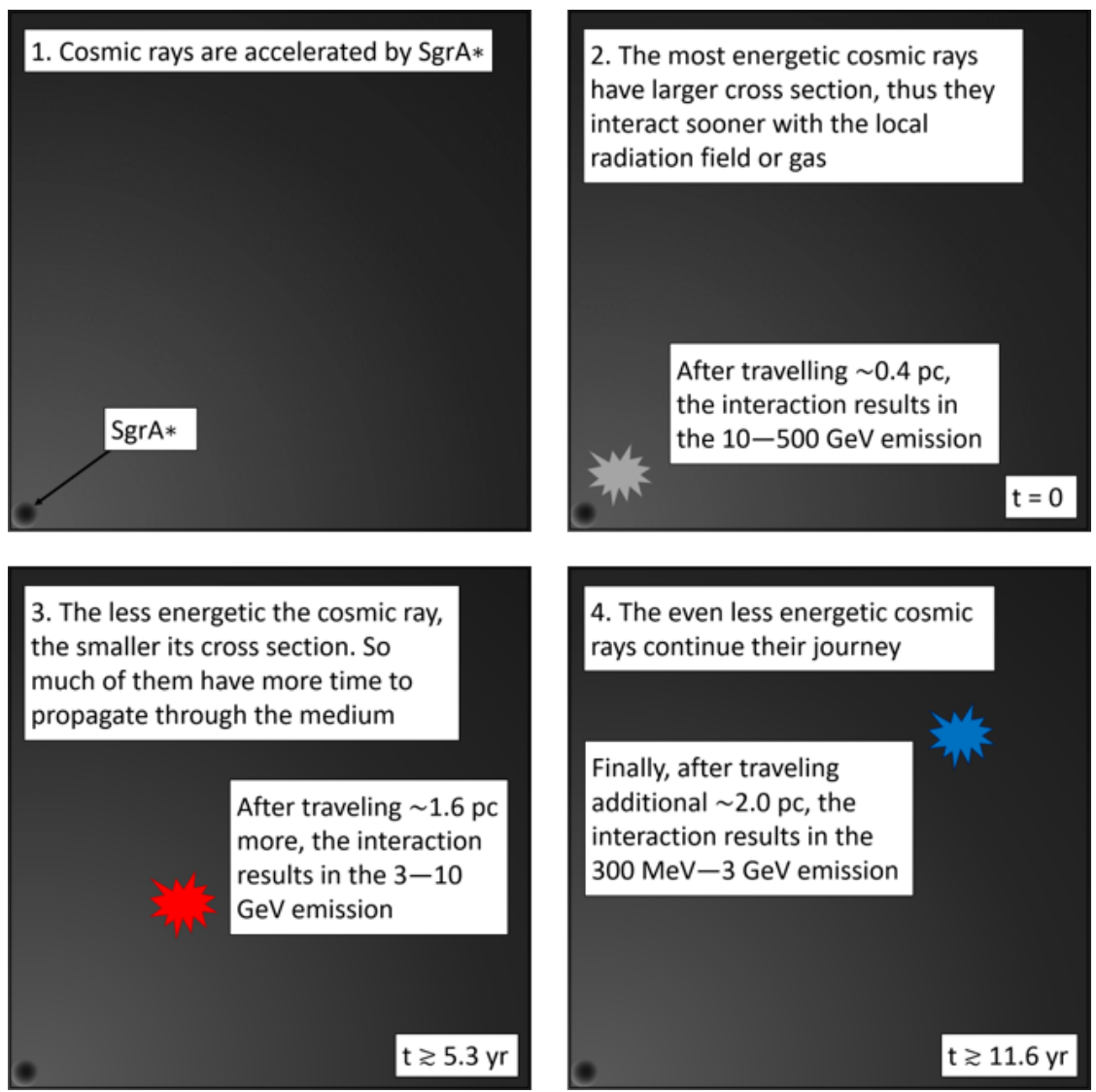

Figure 4.6: A cartoonistic version of the simple model suggested in the text. Cosmic rays accelerated by Sgr A* propagate through the GC region, interacting either with the radiation field or the local gas. More energetic cosmic rays have larger cross sections, thus they interact closer do Sgr A*. Less energetic ones have more time to travel before interacting. The time lengths exhibited at the bottom right part of the panels correspond to the approximate light crossing time for the distances indicated in the Figure text and were chosen based on the centroids distances to Sgr A* (see Figure 3.3).

In Figures 4.7 and 4.8 we show the energy and photon fluxes, respectively, of the three narrower energy bands models. We displaced the data according to the distance between the emission centroids in each energy band. We calculated it based on their angular distances and considering that they are at the GC. We, then, converted this distances into 
time delays, defined as the light crossing time between the centroids separations. These delays are only lower bounds, since the cosmic rays, although relativistic, will take longer than light to spread through the GC. We started by displacing the 3-10 GeV data by $\approx 5.3$ years. This corresponds to the light crossing time of the distance of $\approx 1.6$ pc between the emission centroids in the $10-500 \mathrm{GeV}$ and in the $3-10 \mathrm{GeV}$ ranges (if they are at the $\mathrm{GC}$ distance). The data for the $300 \mathrm{MeV}-3 \mathrm{GeV}$ LC was displaced by $\approx 11.6$ years with the addition of the light crossing time for $1.9 \mathrm{pc}$ (the distance between the $3-10 \mathrm{GeV}$ and the $300 \mathrm{MeV}-3 \mathrm{GeV}$ centroids). It means that fluctuations in the LCs caused by one population of cosmic rays in a panel will be approximately aligned with the potential variability generated by the same population in another panel. Because of the estimated time delays, we changed the horizontal axis to account for the time (in years) elapsed since the beginning of the data collection.
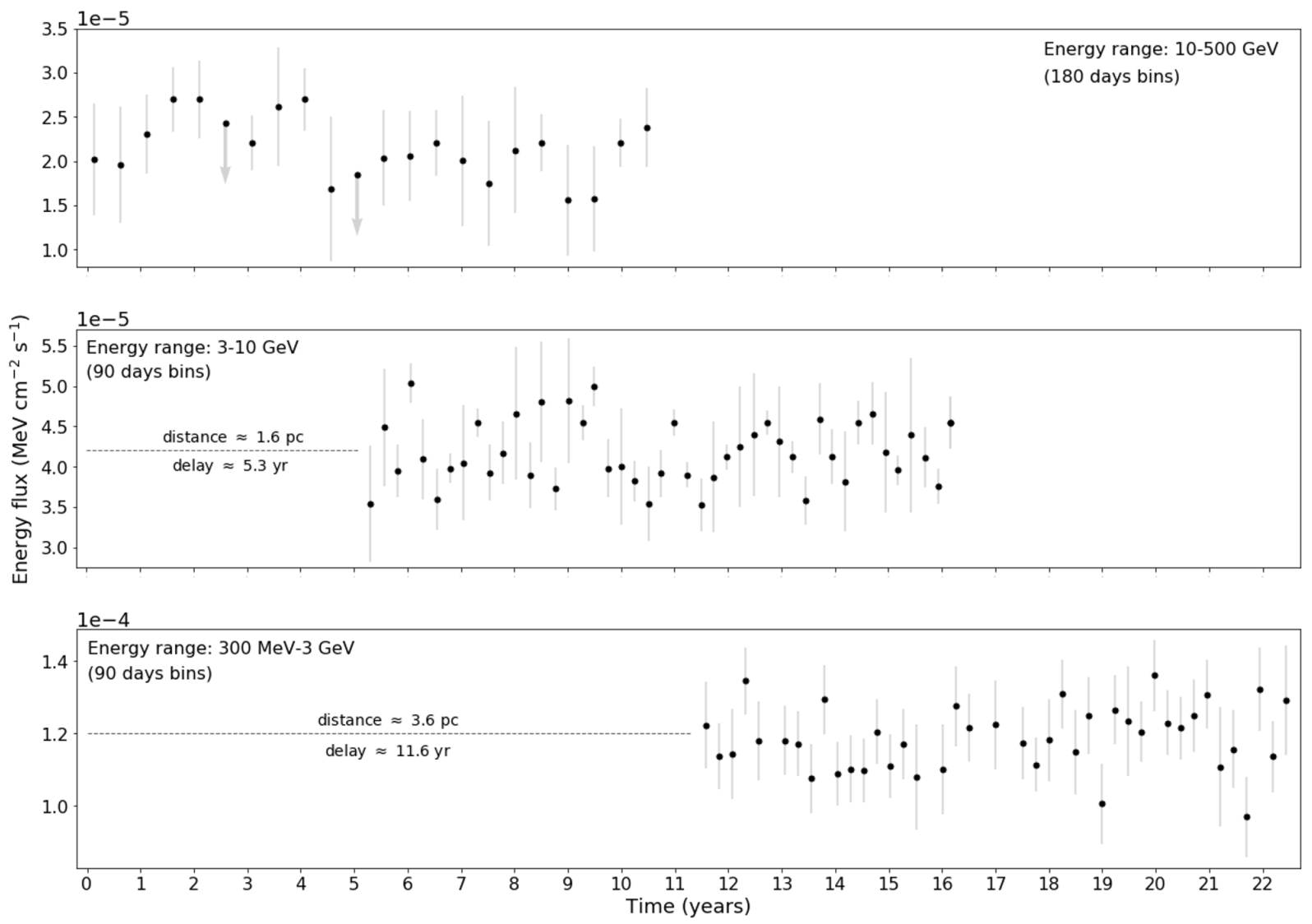

Figure 4.7: We show the energy flux LCs obtained for the narrower energy bands models used in this work: 10-500 GeV (with 180 days bins, in the upper panel), 3-10 GeV (with 90 days bins, in the central panel), and $300 \mathrm{MeV}-3 \mathrm{GeV}$ (with 90 days bins, in the lower panel). The LC in the central panel was displaced by $\approx 5.3$ years, corresponding to the light crossing time between the emission centroids in the $10-500 \mathrm{GeV}$ and the $3-10 \mathrm{GeV}$ energy bands. The displacement in the lower panel is of $\approx 11.6$ years to include the separation between the $3-10 \mathrm{GeV}$ and the $300 \mathrm{MeV}-3 \mathrm{GeV}$ centroids. 

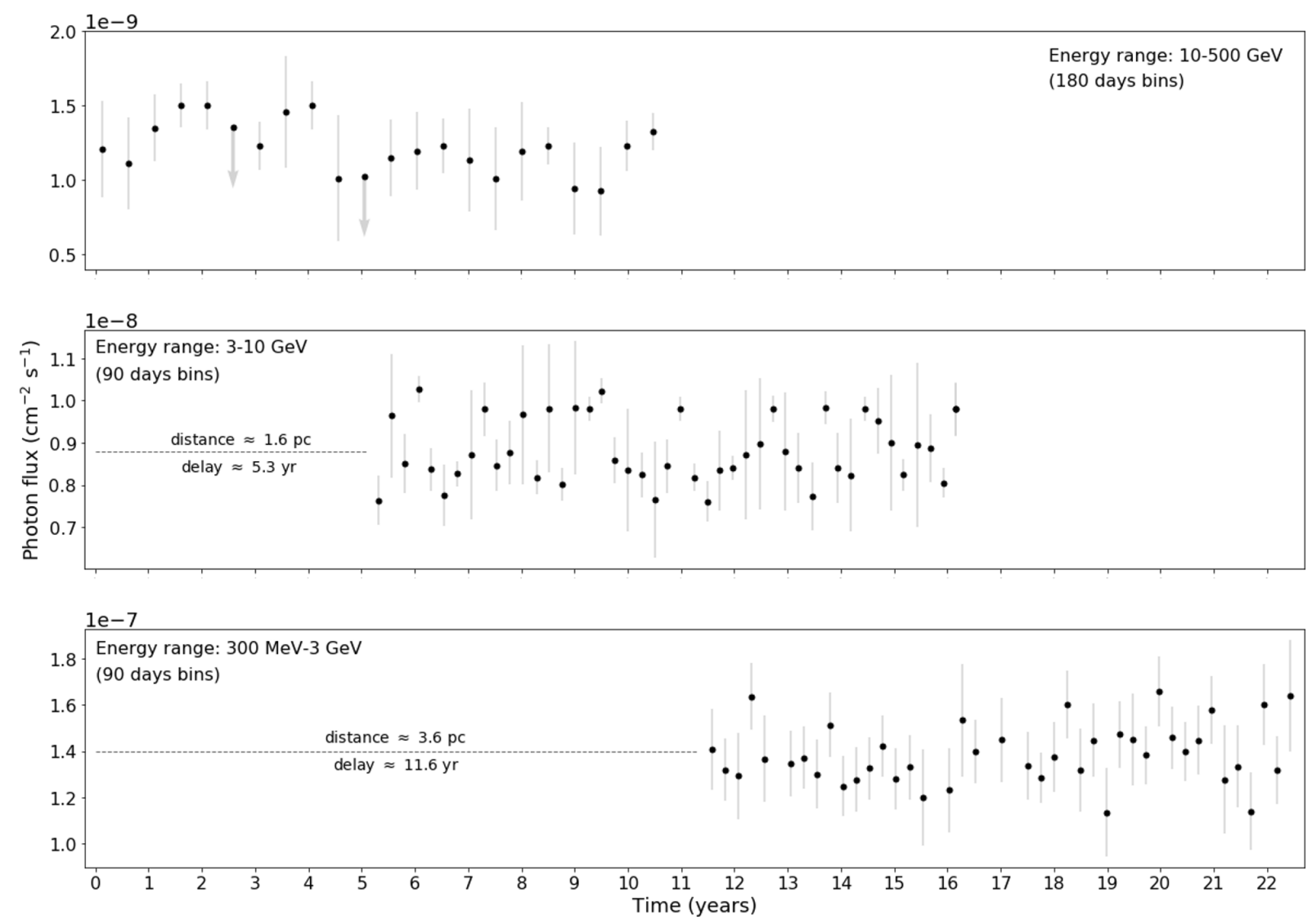

Figure 4.8: We show the photon flux LCs obtained for the narrower energy bands models used in this work: $10-500 \mathrm{GeV}$ (upper panel), 3-10 GeV (central panel), and $300 \mathrm{MeV}-3 \mathrm{GeV}$ (lower panel). The bins sizes and displacements are the same as in Figure 4.7

A visual inspection of Figures 4.7 and 4.8 is enough to rule out any detection of "echoes". This is not surprising for two main reasons. The first is that the variability in these LCs are very similar with a random noise (although we did not test if these fluxes distributions are compatible with a Gaussian random process, as explained in Sections 2.5.3 and 3.3.2, which impose severe limitation on the possibility of correlating variation. Also, since we had to use large time bins in these LCs, the number of data points available to correlate is very limited. 


\subsection{SED and emission models}

In Figure 4.9 we compare the GC source's SED (presented in 3.19) with several models for the MeV to TeV emission from Sgr A*. The list includes the "Fermi-era" models and also a model from Ballantyne et al. (2011) in the TeV energy range, with only a minor overlap with the energy range used in our analysis. A quick comment about this model is that its spectrum is a result of intermittent CR acceleration close to Sgr A* at specific periods in the past. One prediction from Ballantyne et al. (2011) is variability in the emission on time scales of $\sim 10$ years. Ahnen, M. L. et al. (2017) find no evidence of this variability in the MAGIC observations. Also, our LCs in the 10-500 GeV energy range (see Figure 3.18), that partially overlaps with the low energy part of Ballantyne et al. (2011) model, show no significant evidence of variability. The "Fermi-era" models were already described in the discussion above (see Sections 4.1.1 and 4.2.1).

Our results are compatible (or close to) all of the models in the $\sim 3-500 \mathrm{GeV}$ energy range. At lower energies, they depart from the "Fermi-era" hadronic models of: Chernyakova et al. (2011), Linden et al. (2012) and Fatuzzo and Melia (2012).

Kusunose and Takahara (2012) propose two parametrizations of their leptonic model, one to explain the TeV emission and another for the Fermi-LAT range. Finally, Guo and Mathews (2012) suggest a hybrid model with the TeV emission being hadronic while the electrons scatter off the soft background photons to the $\mathrm{MeV}-\mathrm{GeV}$ energy range via IC. Our data are consistent with the low energy components of both models in the $\sim 2-500$ GeV energy range.

Although our data is not totally coincident with any of the models, it is important to notice that they were constructed around Fermi-LAT data obtained during the 2FGL Catalog period. In our work, besides the much longer data collection period (which leads to better statistics), we are using a more recent version of Fermitools, Pass 8 response

\footnotetext{
${ }^{2}$ Figure 4.9 is an adaptation of a plot from Ahnen, M. L. et al. (2017). Originally, the Fermi-LAT data of this image was from Malyshev et al. (2015), but we replaced this with ours. Malyshev et al. (2015) data was created based on the 2FGL J1745.6-2858 source. Later, as discussed in Section 4.2.1 this source was split into two in the following versions of Fermi Catalogs. For this reason, their $E^{2} d N / d E$ values are slightly higher than ours, especially in lower energies. Another reason to explain this difference is the improvement of the galdiff model through the years (Section 1.5.5.1). Malyshev et al. (2015) results mighty be more contaminated with the diffuse emission associated with gas in the Galactic disk than ours.
} 


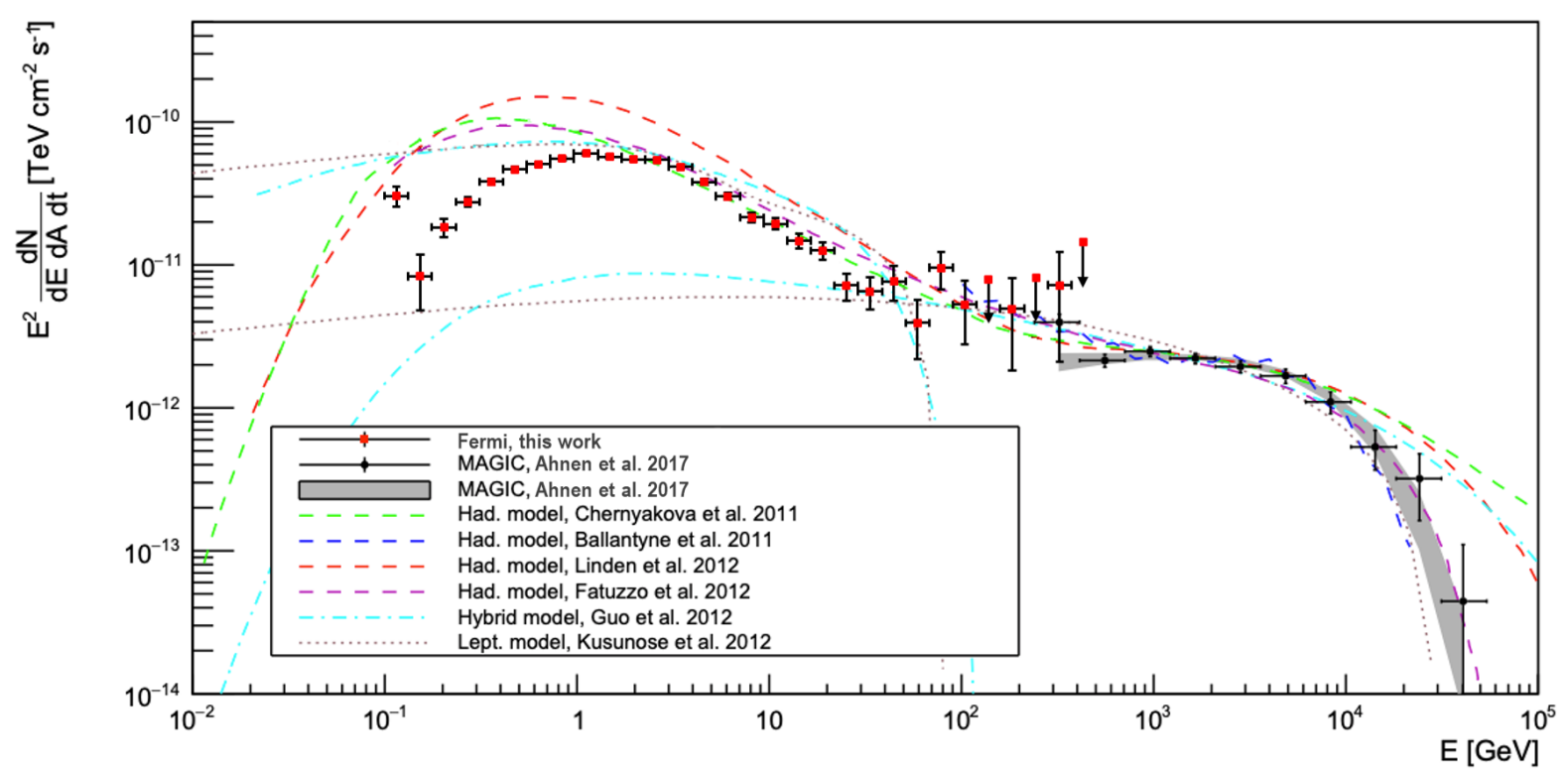

Figure 4.9: The 4FGL J1745.6-2859 SED created for the Universal Model (100 MeV-500 GeV) compared with several models for Sgr A* high energy emission. Source: adapted from Ahnen, M. L. et al. (2017).

functions and the preliminary version of 4FGL catalog (accompanied with improved versions of the diffuse models), all of which were released after these works were published. These improvements allow for much better discrimination between sources, especially the diffuse emission so prominent in the GC and in lower energies. Contamination of diffuse emission in our data is probably the culprit of our lowest energy point being displaced from the spectral model used for the source. This is easier to observe in Figure 3.19, where we also plot the spectral model.

Other than trying to find models that fit exactly to the data, we should be more interested in observing the shape of the observed and the theoretical SEDs. Every hadronic model exhibit a low-energy cut-off (Chernyakova et al., 2011; Linden et al., 2012; Fatuzzo and Melia, 2012). This is expected because of the energy threshold of the "pion-decay bump", described in Section 1.6.2. And this feature is very clear in our data (except for the lowest energy bin). This SED shape is reported for the first time in this thesis and is a strong evidence of a hadronic origin for this emission. The previous versions of the galdiff and Fermi-LAT's response function probably hindered the best assessment of the source's SED in energies $\lesssim$ a few GeV (e.g., Chernyakova et al. 2011; Malyshev et al. 2015; and our own unpublished former studies).

As explained in Section 1.6.2, the "pion-decay bump" is a signature expected in RIAFs. 
In these environments, the protons are heated to very high temperatures, which leads to proton-proton collisions and, consequently, gamma-ray emission. For several decades, RIAFs models have been successfully used to explain Sgr A*'s observations (e.g. Narayan et al. 1995; Yuan et al. 2003, 2004) and our results fit nicely with this framework.

On the high energy end of our SED ( $~ 20 \mathrm{GeV}$ photons), there is a considerable detachment from the log-parabola spectral model used in the analysis (Figure 3.19). The risk of contamination from nearby sources is low either because of the scarcity of candidates and also because of the intrinsically better PSF in higher energies (see the top row of Figure 1.14). In these energies, the Fermi-LAT data transition smoothly to the MAGIC data, as depicted in Figure 4.9, following the behavior of every "Fermi-era" hadronic model (including the hadronic component of the hybrid model from Guo and Mathews 2012); of the Ballantyne et al. (2011) hadronic model that only covers energies $\gtrsim 100 \mathrm{GeV}$; and also of the high energy parametrization of the Kusunose and Takahara (2012) leptonic model.

The two-component spectrum at $\sim \mathrm{GeV}$ and $\sim \mathrm{TeV}$ energies is explained by the hadronic models with very similar arguments. The Chernyakova et al. (2011) spectrum is explained by the different effective velocities of the relativistic and non-relativistic protons accelerated near Sgr A*. Equivalently, Linden et al. (2012) explain their spectrum shape as an outcome of the way protons may propagate through the GC region: either rectilinearly or diffusively and undergoing many or fewer than one collision with the surrounding gas. At the $\sim 10$ $\mathrm{TeV}$, the emission is dominated by a high energy proton population which propagates rectilinearly, while the $\sim \mathrm{GeV}$ emission is governed by less energetic protons propagating diffusively and undergoing Poissonian interactions. A related reason for the observed spectrum is proposed by the two-phase environment of Fatuzzo and Melia (2012). All these scenarios are compatible with the shape and the data of our $\mathrm{SED} \gtrsim$ tens $\mathrm{GeV}$.

As a preliminary assessment of the robustness of our results, we inspected the 7 SEDs created based on different selection of free parameters (see Section 2.6). The "pion-decay bump" is conspicuous in all of them. This indicates that the feature in the SED is not a result of the fitting process. Other robustness tests are also possible, such as using alternative models for the Galactic diffuse gamma-ray emission to compare the resulting SED. These tests will be the performed in the near future.

We will discuss the SED in more detail in a forthcoming publication. 
Chapter 5

\section{Conclusions}

In this Section, we summarize the results of this thesis regarding the imaging, spectral and variability properties of 4FGL J1745.6-2859.

The spatial coincidence of 4FGL J1745.6-2859 with Sgr A* is a necessary but not sufficient condition for it to be considered the counterpart emission of the GC's SMBH. In this respect, our imaging analysis showed that the source's position is coincident with Sgr A*'s in the 60-300 MeV and in the 10-500 GeV energy bands. Also, when we consider only the three highest energy bands used in this work, the emission centroid approaches Sgr A*'s position as the photon energy increases. Furthermore, 4FGL J1745.6-2859's luminosity in the $100 \mathrm{MeV}-500 \mathrm{GeV}$ is also similar to Sgr A*'s bolometric luminosity. The temporal analyses showed no variability in 15 days timescales. And the SED is compatible with a hadronic scenario. Taken together, these results support the picture in which the point source 4FGL J1745.6-2859 observed by Fermi-LAT at the GC is the manifestation of $\mathrm{Sgr} \mathrm{A} *$ in the $\mathrm{MeV}-\mathrm{GeV}$ range.

\subsection{The evidence points to Sagittarius A*}

Our analysis of 4FGL J1745.6-2859 was split into three approaches. First, we evaluated its position (and extension) as function of the photon energy, then we studied its time evolution and, finally, we assessed its spectrum. Taken together, these analyses allow us to conclude that $\mathrm{Sgr} \mathrm{A} *$ is the most likely candidate to explain 4FGL J1745.6-2859 and its properties.

The positional and energetics analyses attained the following four main results: (i) the 
energetics of 4FGL J1745.6-2859 is comparable to the radio-to-X-ray bolometric luminosity of Sgr A*, (ii) among all the possible candidates for the gamma-ray flux of the point source, only the models invoking cosmic rays accelerated by Sgr A* or a nearby source can explain the observations, (iii) the emission centroid moves toward the position of Sgr $\mathrm{A} *$ as the energy is increased and is spatially associated with the CND, a region rich in dense molecular clouds and warm dust, and (iv) other processes not associated with the SMBH could be contributing to the flux at energies $<300 \mathrm{MeV}$ (e.g. pulsars) due to the larger positional uncertainty of the source as the instrument PSF becomes larger at lower energies.

These results suggest that particles responsible for the gamma-ray detected in the three higher energy bands are accelerated by the same process that originates in the region of Sgr A*. A natural consequence of large emission regions is little temporal variability.

The time evolution of the source, observed for $\sim 11.3$ years, shows no signal of variability on 15 days timescales. This suggests that the source is steady in gamma-rays, hinting at a different emission process from the intrinsically variable ones in lower energies (Xrays, NIR and radio). We can not confidently rule out the possibility, though, that these processes are indeed associated but any variability in gamma-rays average out in the 15 days timescales of our bins. Although there are leptonic models constructed over a stable gamma-ray emission from $\mathrm{Sgr} \mathrm{A} *$, this behavior is better explained by hadronic processes. They result in inherently unvarying LCs since the emission regions are larger (than in leptonic scenarios) and the cooling time is much longer for protons.

Another strong evidence favoring hadronic process as the origin of the Fermi-LAT emission from the GC arises from its SED. It clearly shows a "pion-decay bump" and its shape is compatible with every "Fermi-era" hadronic model. The observed "pion-decay bump" is also compatible with the RIAF interpretation of Sgr A*, which is largely used in the literature, since RIAF's environments favor proton-proton collisions and subsequent gamma-ray emission through pion decay.

Furthermore, we scrutinized all the potential candidates for the gamma-ray emission from the GC found in the literature. Only models that link the emission with Sgr A* are compatible with our results. Other candidates are likely to contribute only at low $(\lesssim 300$ $\mathrm{MeV}$ ) energies.

Taken together, the evidence we gathered regarding 4FGL J1745.6-2859 emission point 
in the direction of Sgr A*. An hadronic model, in which the accelerated protons interact with the gas content of the CND, is the most suitable: it naturally explains the steady LCs and the shape of the observed SED. Also, it is compatible with the emission positions and extension ULs.

There are still uncertainties regarding the association of 4FGL J1745.6-2859 with the SMBH. In the following Section we discuss future perspectives concerning our work with Fermi-LAT data and the advent of modern gamma-ray detectors, for the GC high energy study.

\subsection{Future perspectives}

Before we discuss the arrival of a new generation of astrophysical gamma-ray observatories and their impact on the high energy emission from the GC, we will focus on improvements in the context of our own work with Fermi-LAT data that could slightly improve the quality of this work.

The most obvious improvement that we could make in our analysis is to use what we learned with the SED. In Figure 3.19 we can clearly observe that the lowest energy point (that covers the 100-133 MeV energy range) is significantly displaced from the trend observed in higher energies. This is likely due to contamination from other sources, facilitated by Fermi-LAT's broader PSF in lower energies and the ubiquity of low energy sources, including the galdiff. Excluding energies $\lesssim 130 \mathrm{MeV}$ from our analysis would help a better fit of the log-parabola spectral model to the data. It is evident from Figure 3.19 that the modeled cutoff in lower energies (represented by the gray line) is displaced from the SED data. We did not perform an analysis with this different energy selection, but we expect a better fitting of the model to the data, evidenced by higher likelihoods. The impact on the source position's reported, for example, in Figure 3.2 would probably be negligible because we only used the Universal Model (100 MeV-500 GeV) to assess 4FGL J1745.6-2859's position in energies $>300 \mathrm{MeV}$ (the position in lower energies was obtained with an independent model). And the SED data is perfectly compatible with the fitted spectral model up to $\sim 50 \mathrm{GeV}$. Any impact on the source's position is expected to the higher energy band of our work (10-50 GeV). But, a combination of factors-Fermi-LAT's better PSF 
at high energies and the lack of other very high energy sources close to the GC-reduce the potential of this impact.

Figure 3.19 also indicates a displacement of the data form the log-parabola spectral model in energies $\gtrsim 50 \mathrm{GeV}$. Excluding photons with these energies would also help in assessing the "pion-decay bump" shape in our work. The analysis focused in this higher energy emission could be performed separately. Different functions could be used in this energy range, such as a power-law and a log-parabola, and the model with the higher likelihood adopted. In Figure 4.9 it is clear that the high energy Fermi-LAT data transition smoothly to the ground-based telescopes (such as the MAGIC data). This is expected since every hadronic model consider that the GC gamma-ray emission is divided into two components and both contribute in the $\sim 100 \mathrm{GeV}$ energies.

We also plan on developing our own emission models, either hadronic and leptonic, based on the SED we obtained. This will allow us to better understand the high energy processes happening in the vicinity of Sgr A* and put constrains on the cosmic ray population of the GC.

The LCs were created with the whole energy range of the Universal Model. Eliminating photons with energies $\lesssim 130 \mathrm{MeV}$, as we proposed above as an improvement for the quality of the SED, could impact the results obtained in the temporal analysis. Since the contaminating photons are likely poorly modeled galdiff's photons, whose emission is expected to be constant in time, further avoiding this foreground emission could potentially evidence some very small-undetected in this work - variability. On the other hand, limiting the energy range also reduces the number of photons available for the analysis. A smaller number of counts in the LCs leads to points with smaller $T S$, which are consequently reported as ULs. The impact of this trade-off between a better analysis with a limited energy range and the consequent decrease of statistics is very hard to quantify. A empirical assessment is the better approach and, given that generating LCs is very time consuming, we let it to the future.

The LCs we presented in this work could also be improved, even if we keep the same energy range used in the Universal Model. We could use slightly smaller time bins in the LCs, as evidenced by the $T S>16$ in most of the data points (see Appendix $\mathrm{M}$ ). An outcome of reducing the bin sizes, is the decrease of the TS because of the fewer photons and consequence lack of statistics. For this reason, the bins sizes could be only moderately 
reduced. If the source is variable in timescales much smaller than the 15 days bins we used in this work, the potential decrease in the bins sizes would not be enough to assess this variability.

The only way to obtain LCs with time bins that are short and also have high enough $T S$ is to collect large amount of photons. Our results are very close to Fermi-LAT limit, which is governed by its effective area and sensitivity. The ground-based gamma-ray observatories have much larger collecting areas but they cover energy ranges higher than Fermi-LAT's; are localized in regions with low exposure to the GC (with the exception of H.E.S.S.); and operate in a very different way than Fermi, requiring an observing campaign to point to the GC. CTA South (located in the Paranal Observatory in the Atacama Desert in Chile) will have very good exposure to the GC, but has the same characteristics as the other ground-based detectors. Proposed gamma-ray space observatories, AMEGO McEnery et al., 2019) and e-ASTROGAM (de Angelis et al., 2018), will cover energy ranges that overlap with Fermi-LAT's low energy limit, bridging the gap to the current generation of hard X-ray instruments. As Fermi, they will cover the whole sky periodically, being constantly exposed to the GC, which, combined with their high sensitivity, will potentially allow to LCs with higher temporal resolutions.

The new generation of gamma-ray telescopes will also have a positive impact on the imaging, positional and extension analyses that we performed. The advent of CTA will allow for a deep exposure of the GC in energies up to $\sim 300 \mathrm{TeV}$. This will permit studies in spatial and spectral details unavailable today, with arc-minute resolution at energies above Fermi-LAT's operational range, potentially enabling a firmer association between the very high-energy point source in the GC with Sgr A* or other nearby candidate. CTA will be able to enhance significantly the assessment of the morphology of $\sim \mathrm{TeV}$ source, allowing for correlations with the gas density distribution in the GC (Linden et al., 2012). This could potentially confirm or deny the hadronic origin for the very high energy gamma-ray emission. Correspondingly, the AMEGO mission and e-ASTROGAM should help to better constrain the properties of the GC emission in the 60-300 MeV energy band and shed light on the contribution of Sgr A* in the low energy portion of our analysis.

Moving away from the gamma-ray detectors, future missions and results focused in the GC will also contribute to the understanding of the high energy processes associated to Sgr A*. The Extremely Large Telescope (ELT) will increase the probability of 
finding S-star cluster members very close to Sgr A* with NIR observations. Recently, Fragione and Loeb (2020) placed an UL on the spin of Sgr A* based on the spatial distribution of the S-stars. ELT observations would help them to even better constrain their estimate. The EHT observational campaign of Sgr A* will also contribute to measure the SMBH spin. This measurement could help to determine the mechanism behind the Fermi-LAT observations of the GC. Leptonic models are dependent of a relatively high spin, necessary to enable the concentration of magnetic field lines and consequent acceleration of electrons to relativistic speeds and gamma-ray emission through IC scattering.

The recent history of astrophysics is conspicuously brimful of discoveries regarding BHs and the GC. In 2017, Rainer Weiss, Kip S. Thorne and Barry C. Barish shared the Nobel Prize in Physics for the direct detection of gravitational waves from the merger of two BHs. Two years later, the first image of a BH silhouette was captured using the EHT. The following year, Andrea Ghez and Reinhard Genzel were awarded the Nobel Prize in Physics for the discovery of Sgr A*. Jointly, Roger Penrose has also received the prize for his theoretical work robustly connecting BHs to the General Theory of Relativity. In this context, the present work serves the purpose of contributing as a building block to our understanding of those intriguing objects and their role in the cosmos. 


\section{Bibliography}

Abazajian, K. N., Horiuchi, S., Kaplinghat, M., Keeley, R. E., and Macias, O. (2020). Strong constraints on thermal relic dark matter from fermi-lat observations of the galactic center. Phys. Rev. D, 102:043012.

Abazajian, K. N. and Kaplinghat, M. (2012). Detection of a gamma-ray source in the Galactic Center consistent with extended emission from dark matter annihilation and concentrated astrophysical emission. Phys. Rev. D, 86(8):083511.

Abbott, B. P., Abbott, R., Abbott, T. D., Acernese, F., Ackley, K., et al. (2017a). Gw170817: Observation of gravitational waves from a binary neutron star inspiral. Phys. Rev. Lett., 119:161101.

Abbott, B. P., Abbott, R., Abbott, T. D., Acernese, F., Ackley, K., et al. (2017b). Multimessenger observations of a binary neutron star merger. The Astrophysical Journal, 848(2):L12.

Abdo, A. A., Ackermann, M., Ajello, M., Allafort, A., Antolini, E., et al. (2010). Fermi large area telescope first source catalog. ApJS, 188(2):405.

Abdo, A. A., Ackermann, M., Ajello, M., Atwood, W. B., Axelsson, M., et al. (2010). The First Fermi Large Area Telescope Catalog of Gamma-ray Pulsars. ApJS, 187(2):460-494.

Acero, F., Ackermann, M., Ajello, M., Albert, A., Atwood, W. B., et al. (2015). Fermi Large Area Telescope Third Source Catalog. ApJS, 218:23.

Acero, F., Aharonian, F., Akhperjanian, A. G., Anton, G., Barres de Almeida, U., et al. (2010). Localizing the VHE $\gamma$-ray source at the Galactic Centre. MNRAS, 402(3):18771882. 
Ackermann, M., Ajello, M., Albert, A., Allafort, A., Atwood, W. B., et al. (2012a). The Fermi Large Area Telescope on Orbit: Event Classification, Instrument Response Functions, and Calibration. ApJS, 203:4.

Ackermann, M., Ajello, M., Albert, A., Atwood, W., Baldini, L., et al. (2017). The Fermi galactic center GeV excess and implications for dark matter. The Astrophysical Journal, 840(1):43.

Ackermann, M., Ajello, M., Atwood, W. B., Baldini, L., Ballet, J., et al. (2012b). FermiLAT Observations of the Diffuse $\gamma$-Ray Emission: Implications for Cosmic Rays and the Interstellar Medium. ApJ, 750(1):3.

Aharonian, F., Akhperjanian, A. G., Anton, G., Barres de Almeida, U., Bazer-Bachi, A. R., et al. (2009). Spectrum and variability of the Galactic center VHE $\gamma$-ray source HESS J1745-290. A\&GA, 503(3):817-825.

Aharonian, F. and Neronov, A. (2005a). High-Energy Gamma Rays from the Massive Black Hole in the Galactic Center. ApJ, 619:306-313.

Aharonian, F. and Neronov, A. (2005b). TeV gamma rays from the galactic center direct and indirect links to the massive black hole in sgr A*. Astrophysics and Space Science, 300(1):255-265.

Aharonian, F., Yang, R., and de Oña Wilhelmi, E. (2019). Massive stars as major factories of Galactic cosmic rays. Nature Astronomy, 3:561-567.

Aharonian, F., Akhperjanian, A. G., Aye, K.-M., Bazer-Bachi, A. R., Beilicke, M., et al. (2004). Very high energy gamma rays from the direction of Sagittarius A*. $A \mathscr{E} A$, 425(1):L13-L17.

Ahnen, M. L., Ansoldi, S., Antonelli, L. A., Antoranz, P., Arcaro, C., et al. (2017). Observations of Sagittarius A* during the pericenter passage of the G2 object with MAGIC. $A \mathscr{G} A, 601: \mathrm{A} 33$.

Ajello, M., Albert, A., Atwood, W., Barbiellini, G., Bastieri, D., et al. (2016). Fermi-lat observations of high-energy $\gamma$-ray emission toward the galactic center. The Astrophysical Journal, 819(1):44. 
Ajello, M., Angioni, R., Axelsson, M., Ballet, J., Barbiellini, G., et al. (2020). The fourth catalog of active galactic nuclei detected by the Fermi Large Area Telescope. The Astrophysical Journal, 892(2):105.

Ajello, M., Atwood, W. B., Baldini, L., Ballet, J., Barbiellini, G., et al. (2017). 3FHL: The Third Catalog of Hard Fermi-LAT Sources. ApJS, 232(2):18.

Akaike, H. (1974). A new look at the statistical model identification. IEEE Transactions on Automatic Control, 19(6):716-723.

Albert, J., Aliu, E., Anderhub, H., Antoranz, P., Armada, A., et al. (2006). Observation of gamma rays from the galactic center with the MAGIC telescope. The Astrophysical Journal, 638(2):L101-L104.

Almeida, I. and Nemmen, R. (2020). Winds and feedback from supermassive black holes accreting at low rates: hydrodynamical treatment. Monthly Notices of the Royal Astronomical Society, 492(2):2553-2571.

Armillotta, L., Krumholz, M. R., Di Teodoro, E. M., and McClure-Griffiths, N. M. (2019). The life cycle of the Central Molecular Zone - I. Inflow, star formation, and winds. Monthly Notices of the Royal Astronomical Society, 490(3):4401-4418.

Atoyan, A. and Dermer, C. D. (2004). TeV Emission from the Galactic Center Black Hole Plerion. ApJ, 617:L123-L126.

Atwood, W., Albert, A., Baldini, L., Tinivella, M., Bregeon, J., et al. (2013). Pass 8: Toward the full realization of the fermi-lat scientific potential. arXiv preprint arXiv:1303.3514.

Atwood, W. B., Abdo, A. A., Ackermann, M., Althouse, W., Anderson, B., et al. (2009). The Large Area Telescope on the Fermi Gamma-Ray Space Telescope Mission. The Astrophysical Journal, 697(2):1071-1102.

Baganoff, F. K., Bautz, M. W., Brandt, W. N., Chartas, G., Feigelson, E. D., et al. (2001). Rapid X-ray flaring from the direction of the supermassive black hole at the Galactic Centre. Nature, 413:45-48. 
Baganoff, F. K., Maeda, Y., Morris, M., Bautz, M. W., Brandt, W. N., et al. (2003). Chandra X-Ray Spectroscopic Imaging of Sagittarius A* and the Central Parsec of the Galaxy. ApJ, 591:891-915.

Balick, B. and Brown, R. L. (1974). Intense sub-arcsecond structure in the galactic center. ApJ, 194:265-270.

Ball, D., Ozel, F., Psaltis, D., and kwan Chan, C. (2016). Particle Acceleration and the Origin Of X-Ray Flares In GRMHD Simulations Of Sgr A*. The Astrophysical Journal, $826(1): 77$.

Ballantyne, D. R., Schumann, M., and Ford, B. (2011). Modelling the time-dependence of the TeV Gamma-ray source at the Galactic Centre. Monthly Notices of the Royal Astronomical Society, 410(3):1521-1526.

Ballet, J., Burnett, T. H., Digel, S. W., and Lott, B. (2020). Fermi Large Area Telescope Fourth Source Catalog Data Release 2. arXiv e-prints, page arXiv:2005.11208.

Barrière, N. M., Tomsick, J. A., Baganoff, F. K., Boggs, S. E., Christensen, F. E., et al. (2014). NuSTAR Detection of High-Energy X-Ray Emission and Rapid Variability From Sagittarius A* Flares. The Astrophysical Journal, 786(1):46.

Becklin, E. E., Gatley, I., and Werner, M. W. (1982). Far-infrared observations of Sagittarius A - The luminosity and dust density in the central parsec of the Galaxy. ApJ, 258:135-142.

Becklin, E. E., Matthews, K., Neugebauer, G., and Willner, S. P. (1978). Infrared observations of the galactic center. I. Nature of the compact sources. ApJ, 219:121-128.

Becklin, E. E. and Neugebauer, G. (1968). Infrared Observations of the Galactic Center. ApJ, 151:145.

Blum, R. D., Ramond, T. M., Conti, P. S., Figer, D. F., and Sellgren, K. (1997). H-Band Spectroscopic Classification of OB Stars. AJ, 113:1855-1859.

Boehle, A., Ghez, A. M., Schodel, R., Meyer, L., Yelda, S., et al. (2016). An Improved Distance and Mass Estimate For Sgr A* From a Multistar Orbit Analysis. The Astrophysical Journal, 830(1):17. 
Bower, G. C., Falcke, H., Sault, R. J., and Backer, D. C. (2002). The spectrum and variability of circular polarization in Sagittarius A* from 1.4 to $15 \mathrm{GHz}$. The Astrophysical Journal, 571(2):843.

Boyce, H., Haggard, D., Witzel, G., Willner, S. P., Neilsen, J., et al. (2019). Simultaneous X-Ray and Infrared Observations of Sagittarius A*s Variability. The Astrophysical Journal, 871(2):161.

Brinkerink, C. D., Falcke, H., Law, C. J., Barkats, D., Bower, G. C., et al. (2015). ALMA and VLA measurements of frequency-dependent time lags in Sagittarius A*: evidence for a relativistic outflow. $A \mathscr{E} A, 576$ :A41.

Broderick, A. E. and Loeb, A. (2005). Imaging bright-spots in the accretion flow near the black hole horizon of Sgr A*. Monthly Notices of the Royal Astronomical Society, $363(2): 353-362$.

Broderick, A. E. and Loeb, A. (2006). Imaging optically-thin hotspots near the black hole horizon of Sgr A* at radio and near-infrared wavelengths. Monthly Notices of the Royal Astronomical Society, 367(3):905-916.

Brown, R. L. (1982). Precessing jets in Sagittarius A: gas dynamics in the central parsec of the galaxy. ApJ, 262:110-119.

Calderon, D., Cuadra, J., Schartmann, M., Burkert, A., and Russell, C. M. P. (2020). Stellar Winds Pump the Heart of the Milky Way. ApJ, 888(1):L2.

Calore, F., Cholis, I., and Weniger, C. (2015). Background model systematics for the fermi GeV excess. Journal of Cosmology and Astroparticle Physics, 2015(03):038-038.

Chen, Z., Gallego-Cano, E., Do, T., Witzel, G., Ghez, A. M., et al. (2019). Consistency of the Infrared Variability of SGR A* over 22 yr. The Astrophysical Journal, 882(2):L28.

Cherenkov Telescope Array Consortium, Acharya, B. S., Agudo, I., Al Samarai, I., Alfaro, R., et al. (2019). Science with the Cherenkov Telescope Array.

Chernyakova, M., Malyshev, D., Aharonian, F. A., Crocker, R. M., and Jones, D. I. (2011). The High-energy, Arcminute-scale Galactic Center Gamma-ray Source. ApJ, 726:60. 
Cholis, I., Evoli, C., Calore, F., Linden, T., Weniger, C., and Hooper, D. (2015). The galactic center $\mathrm{GeV}$ excess from a series of leptonic cosmic-ray outbursts. Journal of Cosmology and Astroparticle Physics, 2015(12):005-005.

Christopher, M. H., Scoville, N. Z., Stolovy, S. R., and Yun, M. S. (2005). HCN and $\mathrm{HCO}+$ Observations of the Galactic Circumnuclear Disk. The Astrophysical Journal, 622(1):346-365.

Clavel, M., Terrier, R., Goldwurm, A., Morris, M. R., Ponti, G., Soldi, S., and Trap, G. (2013). Echoes of multiple outbursts of Sagittarius A* revealed by Chandra. $A \mathscr{E} A$, 558:A32.

Compton, A. H. (1923). A quantum theory of the scattering of x-rays by light elements. Phys. Rev., 21:483-502.

Crocker, R. M., Fatuzzo, M., Jokipii, J. R., Melia, F., and Volkas, R. R. (2005). The AGASA and SUGAR Anisotropies and TeV Gamma Rays from the Galactic Center: A Possible Signature of Extremely High Energy Neutrons. ApJ, 622:892-909.

D'Agostino, R. (1971). An omnibus test of normality for moderate and large size samples. Biometrika, 58(2):341-348.

D'Agostino, R. and Pearson, E. S. (1973). Tests for departure from normality. Empirical results for the distributions of b2 and $\sqrt{b 1}$. Biometrika, 60(3):613-622.

de Angelis, A. and Mallamaci, M. (2018). Gamma-ray astrophysics. European Physical Journal Plus, 133(8):324.

de Angelis, A., Tatischeff, V., Grenier, I. A., McEnery, J., Mallamaci, M., et al. (2018). Science with e-ASTROGAM. A space mission for MeV-GeV gamma-ray astrophysics. Journal of High Energy Astrophysics, 19:1-106.

de Menezes, R., Cafardo, F., and Nemmen, R. (2019). Milky Way globular clusters in Gamma-rays: analysing the dynamical formation of millisecond pulsars. Monthly Notices of the Royal Astronomical Society, 486(1):851-867.

de Menezes, R., Nemmen, R., Finke, J. D., Almeida, I., and Rani, B. (2020). Gamma-ray observations of low-luminosity active galactic nuclei. MNRAS, 492(3):4120-4130. 
Dermer, C. and Menon, G. (2009). High Energy Radiation from Black Holes: Gamma Rays, Cosmic Rays, and Neutrinos. Princeton Series in Astrophysics. Princeton University Press.

Di Mauro, M. (2021). The characteristics of the Galactic center excess measured with 11 years of Fermi-LAT data. arXiv e-prints, page arXiv:2101.04694.

Dibi, S., Markoff, S., Belmont, R., Malzac, J., Barrière, N. M., and Tomsick, J. A. (2014). Exploring plasma evolution during Sagittarius A* flares. MNRAS, 441:1005-1016.

Do, T., Ghez, A. M., Morris, M. R., Yelda, S., Meyer, L., et al. (2009). A Near-Infrared Variability Study of the Galactic Black Hole: A Red Noise Source With No Detected Periodicity. The Astrophysical Journal, 691(2):1021-1034.

Do, T., Lu, J. R., Ghez, A. M., Morris, M. R., Yelda, S., et al. (2013). Stellar populations in the central $0.5 \mathrm{pc}$ of the galaxy. i. a new method for constructing luminosity functions and surface-density profiles. The Astrophysical Journal, 764(2):154.

Do, T., Witzel, G., Gautam, A. K., Chen, Z., Ghez, A. M., et al. (2019). Unprecedented Near-infrared Brightness and Variability of Sgr A*. The Astrophysical Journal Letters, 882(2):L27.

Dodds-Eden, K., Gillessen, S., Fritz, T. K., Eisenhauer, F., Trippe, S., et al. (2011). The Two States of Sgr A* In The Near-Infrared: Bright Episodic Flares on Top Of Low-Level Continuous Variability. The Astrophysical Journal, 728(1):37.

Dodds-Eden, K., Porquet, D., Trap, G., Quataert, E., Haubois, X., et al. (2009). Evidence for X-Ray Synchrotron Emission from Simultaneous Mid-Infrared to X-Ray Observations of a Strong Sgr A* Flare. ApJ, 698:676-692.

Eckart, A., Baganoff, F., Morris, M., Bautz, M., Brandt, W. N., et al. (2004). First simultaneous NIR/X-ray detection of a flare from Sgr A*. Astronomy \& Astrophysics, 427(1):1-11.

Eckart, A., Baganoff, F., Morris, M., Kunneriath, D., Zamaninasab, M., et al. (2009). Modeling mm-to X-ray flare emission from Sagittarius A*. Astronomy \& Astrophysics, 500(3):935-946. 
Eckart, A., García-Marín, M., Vogel, S., Teuben, P., Morris, M., et al. (2012). Millimeter to X-ray flares from Sagittarius A*. Astronomy \& Astrophysics, 537:A52.

Eckart, A., Hüttemann, A., Kiefer, C., Britzen, S., Zajaček, M., et al. (2017). The Milky Way's Supermassive Black Hole: How Good a Case Is It? Foundations of Physics, 47(5):553-624.

Eckart, A., Schödel, R., García-Marín, M., Witzel, G., Weiss, A., et al. (2008). Simultaneous NIR/sub-mm observation of flare emission from Sagittarius A*. Astronomy \& Astrophysics, 492(2):337-344.

Eckart, A., Schodel, R., Meyer, L., Trippe, S., Ott, T., and Genzel, R. (2006). Polarimetry of near-infrared flares from Sagittarius A*. A\& A, 455(1):1-10.

Eckart, A., Zajacek, M., Parsa, M., Fazeli, E. H. N., Busch, G., et al. (2018). The Multifrequency Behavior of Sagittarius A*. arXiv e-prints, page arXiv:1806.00284.

Eisenhauer, F., Genzel, R., Alexander, T., Abuter, R., Paumard, T., et al. (2005). SINFONI in the Galactic Center: Young Stars and Infrared Flares in the Central LightMonth. The Astrophysical Journal, 628(1):246-259.

Event Horizon Telescope Collaboration et al. (2019). First M87 Event Horizon Telescope Results. I. The Shadow of the Supermassive Black Hole. ApJ, 875(1):L1.

Falcke, H., Goss, W. M., Matsuo, H., Teuben, P., Zhao, J.-H., and Zylka, R. (1998). The Simultaneous Spectrum of Sagittarius A* from 20 Centimeters to 1 Millimeter and the Nature of the Millimeter Excess. The Astrophysical Journal, 499(2):731-734.

Fatuzzo, M. and Melia, F. (2003). A Kinship between the EGRET Supernova Remnants and Sagittarius A East. The Astrophysical Journal, 596(2):1035-1043.

Fatuzzo, M. and Melia, F. (2012). Diffusive Cosmic-Ray Acceleration in Sagittarius A*. ApJ, 757(1):L16.

Fazio, G. G., Hora, J. L., Witzel, G., Willner, S. P., Ashby, M. L. N., et al. (2018). Multiwavelength Light Curves of Two Remarkable Sagittarius A* Flares. ApJ, 864(1):58.

Foreman-Mackey, D., Hogg, D. W., Lang, D., and Goodman, J. (2013). emcee: The MCMC Hammer. PASP, 125(925):306. 
Fragione, G. and Loeb, A. (2020). An Upper Limit on the Spin of SgrA* Based on Stellar Orbits in Its Vicinity. The Astrophysical Journal, 901(2):L32.

Freitag, M., Amaro-Seoane, P., and Kalogera, V. (2006). Stellar remnants in galactic nuclei: Mass segregation. The Astrophysical Journal, 649(1):91-117.

Genzel, R., Eisenhauer, F., and Gillessen, S. (2010). The Galactic Center massive black hole and nuclear star cluster. Reviews of Modern Physics, 82:3121-3195.

Genzel, R., Hollenbach, D., and Townes, C. H. (1994). The nucleus of our galaxy. Reports on Progress in Physics, 57(5):417-479.

Genzel, R., Pichon, C., Eckart, A., Gerhard, O. E., and Ott, T. (2000). Stellar dynamics in the Galactic Centre: proper motions and anisotropy. Monthly Notices of the Royal Astronomical Society, 317(2):348-374.

Genzel, R., Schödel, R., Ott, T., Eckart, A., Alexander, T., Lacombe, F., et al. (2003). Near-infrared flares from accreting gas around the supermassive black hole at the Galactic Centre. Nature, 425:934-937.

Genzel, R., Schodel, R., Ott, T., Eisenhauer, F., Hofmann, R., et al. (2003). The Stellar Cusp around the Supermassive Black Hole in the Galactic Center. The Astrophysical Journal, 594(2):812-832.

Genzel, R., Thatte, N., Krabbe, A., Kroker, H., and Tacconi-Garman, L. E. (1996). The dark mass concentration in the central parsec of the milky way. The Astrophysical Journal, 472(1):153-172.

Ghez, A. M., Salim, S., Weinberg, N. N., Lu, J. R., Do, T., et al. (2008). Measuring Distance and Properties of the Milky Way's Central Supermassive Black Hole with Stellar Orbits. ApJ, 689:1044-1062.

Ghez, A. M., Wright, S. A., Matthews, K., Thompson, D., Le Mignant, D., et al. (2004). Variable Infrared Emission from the Supermassive Black Hole at the Center of the Milky Way. ApJ, 601:L159-L162. 
Gillessen, S., Eisenhauer, F., Fritz, T. K., Bartko, H., Dodds-Eden, K., et al. (2009). The Orbit of the Star S2 Around Sgr A* From Very Large Telescope and Keck Data. The Astrophysical Journal, 707(2):L114-L117.

Gillessen, S., Eisenhauer, F., Trippe, S., Alexander, T., Genzel, R., et al. (2009). Monitoring Stellar Orbits Around the Massive Black Hole in the Galactic Center. ApJ, 692:1075-1109.

Gillessen, S., Genzel, R., Fritz, T. K., Eisenhauer, F., Pfuhl, O., et al. (2013). Pericenter Passage of the Gas Cloud G2 in the Galactic Center. ApJ, 774:44.

Gillessen, S., Genzel, R., Fritz, T. K., Quataert, E., Alig, C., et al. (2012). A gas cloud on its way towards the supermassive black hole at the Galactic Centre. Nature, 481:51-54.

Goodenough, L. and Hooper, D. (2009). Possible evidence for dark matter annihilation in the inner milky way from the fermi gamma ray space telescope. arXiv preprint arXiv:0910.2998.

Goss, W. M., Brown, R. L., and Lo, K. Y. (2003). The Discovery of Sgr A*. Astronomische Nachrichten Supplement, 324(1):497-504.

GRAVITY Collaboration et al. (2018a). Detection of orbital motions near the last stable circular orbit of the massive black hole SgrA*. A\&A, 618:L10.

GRAVITY Collaboration et al. (2018b). Detection of the gravitational redshift in the orbit of the star S2 near the Galactic centre massive black hole. A $\mathscr{G} A, 615:$ L15.

GRAVITY Collaboration et al. (2019). A geometric distance measurement to the galactic center black hole with $0.3 \%$ uncertainty. $A \mathscr{E} A, 625: \mathrm{L} 10$.

GRAVITY Collaboration et al. (2020). Detection of the schwarzschild precession in the orbit of the star s2 near the galactic centre massive black hole. $A \mathscr{E} A$, 636:L5.

Grenier, I., Casandjian, J.-M., and Terrier, R. (2005). Unveiling extensive clouds of dark gas in the solar neighborhood. Science (New York, N.Y.), 307:1292-5.

Guesten, R., Genzel, R., Wright, M. C. H., Jaffe, D. T., Stutzki, J., and Harris, A. I. (1987). Aperture Synthesis Observations of the Circumnuclear Ring in the Galactic Center. ApJ, 318:124. 
Guo, F. and Mathews, W. G. (2012). The Fermi Bubbles. I. Possible Evidence for Recent AGN Jet Activity in the Galaxy. ApJ, 756:181.

Guo, Y.-Q., Yuan, Q., Liu, C., and Li, A.-F. (2013). A hybrid model of GeV-TeV gamma ray emission from the Galactic center. Journal of Physics G Nuclear Physics, 40(6):065201.

Gusten, R. and Downes, D. (1980). Formaldehyde in the Galactic Center region: interpretation. $A \mathscr{G} A, 87: 6-19$.

Gutiérrez, E. M., Nemmen, R., and Cafardo, F. (2020). A Nonthermal Bomb Explains the Near-infrared Superflare of Sgr A*. The Astrophysical Journal, 891(2):L36.

Habibi, M., Gillessen, S., Martins, F., Eisenhauer, F., Plewa, P. M., et al. (2017). Twelve Years of Spectroscopic Monitoring in the Galactic Center: The Closest Look at S-stars near the Black Hole. The Astrophysical Journal, 847(2):120.

Haggard, D., Nynka, M., Mon, B., de la Cruz Hernandez, N., et al. (2019). Chandra Spectral and Timing Analysis of Sgr A*'s Brightest X-Ray Flares. ApJ, 886(2):96.

Hales, C. A., Gaensler, B. M., Chatterjee, S., van der Swaluw, E., and Camilo, F. (2009). A Proper Motion for the Pulsar Wind Nebula G359.23-0.82, the "Mouse," Associated with the Energetic Radio Pulsar J1747-2958. ApJ, 706(2):1316-1322.

Hartman, R. C., Bertsch, D. L., Bloom, S. D., Chen, A. W., Deines-Jones, P., et al. (1999). The Third EGRET Catalog of High-Energy Gamma-Ray Sources. The Astrophysical Journal Supplement Series, 123(1):79-202.

Hawley, J. F. and Balbus, S. A. (2002). The Dynamical Structure of Nonradiative Black Hole Accretion Flows. ApJ, 573:738-748.

Heckman, T. M. and Best, P. N. (2014). The Coevolution of Galaxies and Supermassive Black Holes: Insights from Surveys of the Contemporary Universe. ARA\& A, 52:589-660.

Herold, L. and Malyshev, D. (2019). Hard and bright gamma-ray emission at the base of the Fermi bubbles. arXiv e-prints, page arXiv:1904.01454.

Herrnstein, R. M., Zhao, J.-H., Bower, G. C., and Goss, W. M. (2004). The Variability of Sagittarius A* at Centimeter Wavelengths. AJ, 127(6):3399-3410. 
H.E.S.S. Collaboration et al. (2016). Acceleration of petaelectronvolt protons in the Galactic Centre. Nature, 531:476-479.

H.E.S.S. Collaboration et al. (2018). Characterising the vhe diffuse emission in the central 200 parsecs of our galaxy with h.e.s.s. $A \mathscr{E} A, 612$ :A9.

Hinton, J. A. and Aharonian, F. A. (2007). Inverse Compton Scenarios for the TeV Gamma-Ray Emission of the Galactic Center. ApJ, 657(1):302-307.

Ho, L. C. (2008). Nuclear Activity in Nearby Galaxies. ARA\&A, 46:475-539.

Hogg, D., Bovy, J., and Lang, D. (2010). Data analysis recipes: Fitting a model to data. ArXiv e-prints.

Hooper, D. and Dingus, B. (2005). Improving the angular resolution of EGRET and new limits on supersymmetric dark matter near the galactic center. Advances in Space Research, 35(1):130-134.

Hooper, D. and Goodenough, L. (2011). Dark matter annihilation in the Galactic Center as seen by the Fermi Gamma Ray Space Telescope. Physics Letters B, 697:412-428.

Hooper, D. and Linden, T. (2011). Origin of the gamma rays from the Galactic Center. Phys. Rev. D, 84(12):123005.

Hooper, D. and Linden, T. (2016). The gamma-ray pulsar population of globular clusters: implications for the gev excess. Journal of Cosmology and Astroparticle Physics, 2016(08):018.

Hora, J. L., Witzel, G., Ashby, M. L. N., Becklin, E. E., and Carey S. et al. (2014). Spitzer/IRAC Observations of the Variability of Sgr A* and the Object G2 at 4.5 microns. The Astrophysical Journal, 793(2):120.

Hornstein, S. D., Matthews, K., Ghez, A. M., Lu, J. R., and Morris, M. et al. (2007). A Constant Spectral Index for Sagittarius A* during Infrared/X-Ray Intensity Variations. The Astrophysical Journal, 667(2):900-910.

Igumenshchev, I. V. and Abramowicz, M. A. (1999). Rotating accretion flows around black holes: convection and variability. MNRAS, 303:309-320. 
Igumenshchev, I. V. and Abramowicz, M. A. (2000). Two-dimensional Models of Hydrodynamical Accretion Flows into Black Holes. ApJS, 130:463-484.

Igumenshchev, I. V., Abramowicz, M. A., and Narayan, R. (2000). Numerical Simulations of Convective Accretion Flows in Three Dimensions. ApJ, 537:L27-L30.

Igumenshchev, I. V., Narayan, R., and Abramowicz, M. A. (2003). Three-dimensional Magnetohydrodynamic Simulations of Radiatively Inefficient Accretion Flows. ApJ, 592:1042-1059.

Jackson, J. M., Geis, N., Genzel, R., Harris, A. I., Madden, S., et al. (1993). Neutral Gas in the Central 2 Parsecs of the Galaxy. ApJ, 402:173.

Kafexhiu, E., Aharonian, F., Taylor, A. M., and Vila, G. S. (2014). Parametrization of gamma-ray production cross sections for $\mathrm{p} p$ interactions in a broad proton energy range from the kinematic threshold to PeV energies. Physical Review D, 90(12):123014.

Karssen, G. D., Bursa, M., Eckart, A., Valencia-S, M., Dovčiak, M., et al. (2017). Bright Xray flares from Sgr A*. Monthly Notices of the Royal Astronomical Society, 472(4):44224433.

Kennea, J. A., Burrows, D. N., Kouveliotou, C., Palmer, D. M., Gougucs, E., et al. (2013). Swift Discovery of a new soft gamma repeater, SGR J1745-29, near Sagittarius A*. The Astrophysical Journal, 770(2):L24.

Koratkar, A. and Blaes, O. (1999). The Ultraviolet and Optical Continuum Emission in Active Galactic Nuclei: The Status of Accretion Disks. PASP, 111:1-30.

Kormendy, J. and Richstone, D. (1995). Inward Bound-The Search For Supermassive Black Holes In Galactic Nuclei. ARA\&A, 33:581.

Kosack, K., Badran, H. M., Bond, I. H., Boyle, P. J., and Bradbury, S. M. et al. (2004). TeV Gamma-Ray Observations of the Galactic Center. The Astrophysical Journal, 608(2):L97-L100.

Kusunose, M. and Takahara, F. (2012). A Leptonic Model of Steady High-energy GammaRay Emission from Sgr A*. ApJ, 748(1):34. 
Lacy, J. H., Townes, C. H., Geballe, T. R., and Hollenbach, D. J. (1980). Observations of the motion and distribution of the ionized gas in the central parsec of the Galaxy. II. ApJ, 241:132-146.

Lacy, J. H., Townes, C. H., and Hollenbach, D. J. (1982). The nature of the central parsec of the Galaxy. ApJ, 262:120-134.

LaRosa, T. N., Kassim, N. E., Lazio, T. J. W., and Hyman, S. D. (2000). A Wide-Field 90 Centimeter VLA Image of the Galactic Center Region. AJ, 119(1):207-240.

Linden, T., Lovegrove, E., and Profumo, S. (2012). The Morphology of Hadronic Emission Models for the Gamma-Ray Source at the Galactic Center. ApJ, 753(1):41.

Lo, K. and Claussen, M. (1983). High-resolution observations of ionized gas in central 3 parsecs of the galaxy: possible evidence for infall. Nature, 306(5944):647-651.

Lynden-Bell, D. (1969). Galactic Nuclei as Collapsed Old Quasars. Nature, 223:690-694.

Macias, O., Gordon, C., Crocker, R. M., Coleman, B., and Paterson, D. et al. (2018). Galactic bulge preferred over dark matter for the Galactic centre gamma-ray excess. Nature Astronomy, 2(5):387-392.

Macquart, J.-P., Bower, G. C., Wright, M. C. H., Backer, D. C., and Falcke, H. (2006). The Rotation Measure and 3.5 Millimeter Polarization of Sagittarius A*. The Astrophysical Journal, 646(2):L111-L114.

MAGIC Collaboration et al. (2020). MAGIC observations of the diffuse $\gamma$-ray emission in the vicinity of the Galactic Centre. arXiv e-prints, page arXiv:2006.00623.

Mahadevan, R., Narayan, R., and Krolik, J. (1997). Gamma-Ray Emission from Advectiondominated Accretion Flows around Black Holes: Application to the Galactic Center. ApJ, 486:268.

Malyshev, D., Chernyakova, M., Neronov, A., and Walter, R. (2015). Leptonic origin of the $100 \mathrm{MeV} \gamma$-ray emission from the Galactic centre. A\& A, 582:A11.

Marrone, D. P., Baganoff, F. K., Morris, M. R., Moran, J. M., and Ghez, A.M. et al. (2008). An X-Ray, Infrared, and Submillimeter Flare of Sagittarius A*. ApJ, 682:373-383. 
Marrone, D. P., Moran, J. M., Zhao, J.-H., and Rao, R. (2006). Interferometric Measurements of Variable $340 \mathrm{GHz}$ Linear Polarization in Sagittarius A*. ApJ, 640:308-318.

Mattox, J. R., Bertsch, D., Chiang, J., Dingus, B., Digel, S., Esposito, J., Fierro, J., Hartman, R., Hunter, S., Kanbach, G., et al. (1996). The likelihood analysis of EGRET data. The Astrophysical Journal, 461:396.

Mauerhan, J. C., Morris, M., Walter, F., and Baganoff, F. K. (2005). Intraday Variability of Sagittarius A* at 3 Millimeters. ApJ, 623:L25-L28.

Mayer-Hasselwander, H. A., Bertsch, D. L., Dingus, B. L., Eckart, A., Esposito, J. A., et al. (1998). High-energy gamma-ray emission from the Galactic Center. A\& A, 335:161-172.

McEnery, J., van der Horst, A., Dominguez, A., Moiseev, A., Marcowith, A., et al. (2019). All-sky Medium Energy Gamma-ray Observatory: Exploring the Extreme Multimessenger Universe. In Bulletin of the American Astronomical Society, volume 51, page 245.

Meyer, L., Do, T., Ghez, A., Morris, M. R., Witzel, G., et al. (2008). A 600 Minute NearInfrared Light Curve of Sagittarius A*. The Astrophysical Journal, 688(1):L17-L20.

Meyer, L., Eckart, A., Schödel, R., Duschl, W. J., Muzi'c, K., et al. (2006a). Nearinfrared polarimetry setting constraints on the orbiting spot model for Sgr A* flares. $A \mathscr{E} A, 460(1): 15-21$.

Meyer, L., Schödel, R., Eckart, A., Duschl, W. J., Karas, V., and Dovciak, M. (2007). On the orientation of the Sagittarius A* system. A\&A, 473(3):707-710.

Meyer, L., Schödel, R., Eckart, A., Karas, V., Dovciak, M., and Duschl, W. J. (2006b). K-band polarimetry of an Sgr A* flare with a clear sub-flare structure. A\&A, 458(2):L25L28.

Mezger, P. G., Duschl, W. J., and Zylka, R. (1996). The Galactic Center: a laboratory for AGN? The Astronomy and Astrophysics Review, 7(4):289-388.

Mezger, P. G. and Wink, J. E. (1986). Dynamics of the ionized gas within 2 PC of the galactic center. I. First results of a radio recombination line survey. A\&A, 157:252-266. 
Miyazaki, A., Tsutsumi, T., and Tsuboi, M. (2004). Intraday Variation of Sagittarius A* at Short Millimeter Wavelengths. The Astrophysical Journal, 611(2):L97-L100.

Miyoshi, M., Moran, J., Herrnstein, J., Greenhill, L., Nakai, N., et al. (1995). Evidence for a black hole from high rotation velocities in a sub-parsec region of NGC4258. Nature, 373:127-129.

Mori, K., Gotthelf, E. V., Zhang, S., An, H., et al. (2013). NuStar Discovery of A 3.76s Transient Magnetar Near Sagittarius A*. The Astrophysical Journal, 770(2):L23.

Mori, M. (2003). CANGAROO Project for High-Energy Gamma-Ray Astrophysics. Progress of Theoretical Physics Supplement, 151:85-94.

Morris, M. and Serabyn, E. (1996). The galactic center environment. Annual Review of Astronomy and Astrophysics, 34(1):645-701.

Morris, M. R., Meyer, L., and Ghez, A. M. (2012). Galactic center research: manifestations of the central black hole. Research in Astronomy and Astrophysics, 12(8):995-1020.

Mouawad, N., Eckart, A., Pfalzner, S., Schodel, R., Moultaka, J., and Spurzem, R. (2005). Weighing the cusp at the Galactic Center. Astron. Nachr., 326:83.

Muno, M. P., Baganoff, F. K., Bautz, M. W., Feigelson, E. D., Garmire, G. P., et al. (2004). Diffuse X-Ray Emission in a DeepChandraImage of the Galactic Center. The Astrophysical Journal, 613(1):326-342.

Murchikova, E. M., Phinney, E. S., Pancoast, A., and Blandford, R. D. (2019). A cool accretion disk around the galactic centre black hole. Nature, 570(7759):83-86.

Narayan, R. (2002). Why Do AGN Lighthouses Switch Off? In Gilfanov, M., Sunyeav, R., and Churazov, E., editors, Lighthouses of the Universe: The Most Luminous Celestial Objects and Their Use for Cosmology, page 405.

Narayan, R., Mahadevan, R., Grindlay, J. E., Popham, R. G., and Gammie, C. (1998). Advection-dominated accretion model of Sagittarius A*: evidence for a black hole at the Galactic center. ApJ, 492:554-568.

Narayan, R. and McClintock, J. E. (2008). Advection-dominated accretion and the black hole event horizon. New Astron. Rev., 51:733-751. 
Narayan, R., Yi, I., and Mahadevan, R. (1995). Explaining the spectrum of Sagittarius A* with a model of an accreting black hole. Nature, 374:623-625.

Navarro, J. F., Frenk, C. S., and White, S. D. M. (1997). A universal density profile from hierarchical clustering. The Astrophysical Journal, 490(2):493-508.

Neilsen, J., Markoff, S., Nowak, M. A., Dexter, J., Witzel, G., et al. (2015). The X-Ray Flux Distribution of Sagittarius A* as Seen by Chandra. ApJ, 799:199.

Neilsen, J., Nowak, M. A., Gammie, C., Dexter, J., Markoff, S., et al. (2013). A Chandra/HETGS Census of X-Ray Variability from Sgr A* during 2012. ApJ, 774:42.

Nolan, P. L., Abdo, A. A., Ackermann, M., Ajello, M., Allafort, A., et al. (2012). Fermi Large Area Telescope Second Source Catalog. ApJS, 199:31.

Nowak, M. A., Neilsen, J., Markoff, S. B., Baganoff, F. K., Porquet, D., et al. (2012). Chandra/HETGS Observations of the Brightest Flare Seen From Sgr A*. The Astrophysical Journal, $759(2): 95$.

Oka, K. and Manmoto, T. (2003). Gamma-ray emission from an accretion flow around a Kerr black hole. MNRAS, 340:543-550.

O'Leary, R. M., Kistler, M. D., Kerr, M., and Dexter, J. (2015). Young pulsars and the galactic center gev gamma-ray excess. arXiv preprint arXiv:1504.0247\%.

Paumard, T., Genzel, R., Martins, F., Nayakshin, S., Beloborodov, A. M., et al. (2006). The Two Young Star Disks in the Central Parsec of the Galaxy: Properties, Dynamics, and Formation. The Astrophysical Journal, 643(2):1011-1035.

Paumard, T., Maillard, J.-P., and Stolovy, S. (2003). New results on the galactic center helium stars. Astronomische Nachrichten, 324(S1):303-307.

Peißker, F., Eckart, A., Zajaček, M., Ali, B., and Parsa, M. (2020). S62 and S4711: Indications of a Population of Faint Fast-moving Stars inside the S2 Orbit-S4711 on a 7.6 yr Orbit around Sgr A*. The Astrophysical Journal, 899(1):50.

Petrov, L., Kovalev, Y. Y., Fomalont, E. B., and Gordon, D. (2011). The Very Long Baseline Array Galactic Plane Survey_VGaPS. AJ, 142(2):35. 
Petrovic, J., Serpico, P. D., and Zaharijaš, G. (2014). Galactic center gammaray "excess" from an active past of the galactic centre? Journal of Cosmology and Astroparticle Physics, 2014(10):052.

Phifer, K., Do, T., Meyer, L., Ghez, A. M., Witzel, G., et al. (2013). Keck Observations of the Galactic Center Source G2: Gas Cloud or Star? ApJ, 773:L13.

Plambeck, R., Dexter, J., Bower, G. C., Kelly, B., Marrone, D. P., and Stone, J. (2014). An $8 \mathrm{~h}$ characteristic time-scale in submillimetre light curves of Sagittarius A*. Monthly Notices of the Royal Astronomical Society, 442(3):2797-2808.

Plewa, P. M., Gillessen, S., Pfuhl, O., Eisenhauer, F., Genzel, R., et al. (2017). The Post-pericenter Evolution of the Galactic Center Source G2. The Astrophysical Journal, $840(1): 50$.

Ponti, G., De Marco, B., Morris, M. R., Merloni, A., Muñoz-Darias, T., et al. (2015). Fifteen years of XMM-Newton and Chandra monitoring of Sgr A*: evidence for a recent increase in the bright flaring rate. MNRAS, 454:1525-1544.

Ponti, G., George, E., Scaringi, S., Zhang, S., Jin, C., and et al. (2017). A powerful flare from Sgr A* confirms the synchrotron nature of the X-ray emission. Monthly Notices of the Royal Astronomical Society, 468(2):2447-2468.

Ponti, G., Terrier, R., Goldwurm, A., Belanger, G., and Trap, G. (2010). Discovery of a Superluminal Fe K Echo at the Galactic Center: The Glorious Past of Sgr A* Preserved by Molecular Clouds. ApJ, 714:732-747.

Porquet, D., Grosso, N., Predehl, P., Hasinger, G., Yusef-Zadeh, F., et al. (2008). X-ray hiccups from Sagittarius A* observed by XMM-Newton-The second brightest flare and three moderate flares caught in half a day. Astronomy \& Astrophysics, 488(2):549-557.

Porquet, D., Predehl, P., Aschenbach, B., Grosso, N., Goldwurm, A., et al. (2003). XMMNewton observation of the brightest X-ray flare detected so far from Sgr A*. Astronomy E Astrophysics, 407(1):L17-L20.

Principe, G., Malyshev, D., Ballet, J., and Funk, S. (2018). The first catalog of Fermi-LAT sources below $100 \mathrm{MeV}$. A\&A, 618:A22. 
Quataert, E. (2003). Radiatively Inefficient Accretion Flow Models of Sgr A*. Astronomische Nachrichten, 324(S1):435-443.

Quataert, E., Dorland, W., and Hammett, G. W. (2002). The Magnetorotational Instability in a Collisionless Plasma. ApJ, 577:524-533.

Quataert, E. and Loeb, A. (2005). Nonthermal THz to TeV emission from stellar wind shocks in the galactic center. The Astrophysical Journal, 635(1):L45-L48.

Reid, M. J. and Brunthaler, A. (2004). The Proper Motion of Sagittarius A*. II. The Mass of Sagittarius A*. The Astrophysical Journal, 616(2):872-884.

Ressler, S. M., Quataert, E., and Stone, J. M. (2018). Hydrodynamic simulations of the inner accretion flow of Sagittarius A* fuelled by stellar winds. Monthly Notices of the Royal Astronomical Society, 478(3):3544-3563.

Rieke, G. H. and Lebofsky, M. J. (1985). The interstellar extinction law from 1 to 13 microns. ApJ, 288:618-621.

Rieke, G. H., Rieke, M. J., and Paul, A. E. (1989). Origin of the Excitation of the Galactic Center. ApJ, 336:752.

Roberts, D. A. and Goss, W. M. (1993). Multiconfiguration VLA H92 alpha Observations of Sagittarius A West at 1 Arcsecond Resolution. ApJS, 86:133.

Rubilar, G. F. and Eckart, A. (2001). Periastron shifts of stellar orbits near the Galactic Center. A\&SA, 374:95-104.

Saitoh, T. R., Makino, J., Asaki, Y., Baba, J., Komugi, S., et al. (2012). Flaring up of the Compact Cloud G2 during the Close Encounter with Sgr A*. ArXiv e-prints.

Sądowski, A., Narayan, R., Penna, R., and Zhu, Y. (2013). Energy, momentum and mass outflows and feedback from thick accretion discs around rotating black holes. MNRAS, $436: 3856-3874$.

Schartmann, M., Burkert, A., Alig, C., Gillessen, S., Genzel, R., and et al. (2012). Simulations of the Origin and Fate of the Galactic Center Cloud G2. ApJ, 755:155. 
Schödel, R., Eckart, A., Alexander, T., Merritt, D., Genzel, R., et al. (2007). The structure of the nuclear stellar cluster of the Milky Way. A\&A, 469:125-146.

Schödel, R., Merritt, D., and Eckart, A. (2009). The nuclear star cluster of the Milky Way: proper motions and mass. $A \mathscr{G} A, 502: 91-111$.

Shah, Z., Mankuzhiyil, N., Sinha, A., Misra, R., Sahayanathan, S., and Iqbal, N. (2018). Log-normal flux distribution of bright Fermi blazars. Research in Astronomy and Astrophysics, 18(11):141.

Shapiro, S. S. and Wilk, M. B. (1965). An analysis of variance test for normality (complete samples). Biometrika, 52(3-4):591-611.

Sinha, A., Shukla, A., Saha, L., Acharya, B. S., Anupama, G. C., Bhattacharjee, P., Britto, R. J., Chitnis, V. R., Prabhu, T. P., Singh, B. B., and Vishwanath, P. R. (2016). Long-term study of mkn 421 with the hagar array of telescopes. A\&̈A, 591:A83.

Stecker, F. W. (1971). Cosmic gamma rays, volume 249. Scientific and Technical Information Office, National Aeronautics and Space.

Stephens, M. A. (1974). Edf statistics for goodness of fit and some comparisons. Journal of the American Statistical Association, 69(347):730-737.

Stone, J. M., Marrone, D. P., Dowell, C. D., Schulz, B., Heinke, C. O., and Yusef-Zadeh, F. (2016). Far Infrared Variability of Sagittarius A*: 25.5 hr of Monitoring with Herschel. The Astrophysical Journal, 825(1):32.

Stone, J. M., Pringle, J. E., and Begelman, M. C. (1999). Hydrodynamical non-radiative accretion flows in two dimensions. MNRAS, 310:1002-1016.

Su, M., Slatyer, T. R., and Finkbeiner, D. P. (2010). Giant Gamma-ray Bubbles from Fermi-LAT: Active Galactic Nucleus Activity or Bipolar Galactic Wind? ApJ, 724:10441082.

Swanenburg, B. N., Bennett, K., Bignami, G., Buccheri, R., Caraveo, P., et al. (1981). Second COS B catalog of high-energy gamma-ray sources. The Astrophysical Journal, 243:L69-L73. 
Tanner, A., Figer, D. F., Najarro, F., Kudritzki, R. P., Gilmore, D., et al. (2006). High Spectral Resolution Observations of the Massive Stars in the Galactic Center. The Astrophysical Journal, 641(2):891-904.

The Fermi-LAT Collaboration (2019). Fermi Large Area Telescope Fourth Source Catalog. arXiv e-prints, page arXiv:1902.10045.

The Fermi-LAT Collaboration et al. (2017). Characterizing the population of pulsars in the inner Galaxy with the Fermi Large Area Telescope. arXiv preprint arXiv:1705.00009.

The IceCube Collaboration, Fermi-LAT, MAGIC, AGILE, ASAS-SN, et al. (2018). Multimessenger observations of a flaring blazar coincident with high-energy neutrino IceCube170922A. Science.

Thompson, C. and Beloborodov, A. M. (2005). High-Energy Emission from Magnetars. ApJ, 634:565-569.

Trap, G., Goldwurm, A., Dodds-Eden, K., Weiss, A., Terrier, R., et al. (2011). Concurrent X-ray, near-infrared, sub-millimeter, and GeV gamma-ray observations of Sagittarius A*. AESA, 528:A140.

Trippe, S., Paumard, T., Ott, T., Gillessen, S., Eisenhauer, F., et al. (2007). A polarized infrared flare from Sagittarius $\mathrm{A}^{*}$ and the signatures of orbiting plasma hotspots*. Monthly Notices of the Royal Astronomical Society, 375(3):764-772.

Tsuchiya, K., Enomoto, R., Ksenofontov, L. T., Mori, M., Naito, T., et al. (2004). Detection of Sub-TeV Gamma Rays from the Galactic Center Direction by CANGAROO-II. ApJ, 606(2):L115-L118.

Uttley, P. and McHardy, I. M. (2001). The flux-dependent amplitude of broadband noise variability in X-ray binaries and active galaxies. MNRAS, 323:L26-L30.

Vasiliev, E. and Zelnikov, M. (2008). Dark matter dynamics in the galactic center. Phys. Rev. D, 78:083506.

Virtanen, P., Gommers, R., Oliphant, T. E., Haberland, M., SciPy 1.0 Contributors, et al. (2020). SciPy 1.0: Fundamental Algorithms for Scientific Computing in Python. Nature Methods, 17:261-272. 
von Fellenberg, S. D., Gillessen, S., Graciá-Carpio, J., Fritz, T. K., Dexter, J., et al. (2018).

A Detection of Sgr A* in the Far Infrared. The Astrophysical Journal, 862(2):129.

Wang, Q. D., Lu, F. J., and Gotthelf, E. V. (2006). G359.95-0.04: an energetic pulsar candidate near Sgr A*. MNRAS, 367(3):937-944.

Wardle, M. (2011). X-ray Echoes of Infrared Flaring in Sgr A*. In Morris, M., Wang, Q., and Yuan, F., editors, Galactic Center, Astronomical Society of the Pacific Conference Series, pages 450-455. Astronomical Society of the Pacific.

Witzel, G., Eckart, A., Bremer, M., Zamaninasab, M., Shahzamanian, B., et al. (2012). Source-intrinsic Near-Infrared Properties of Sgr A*: Total Intensity Measurements. The Astrophysical Journal Supplement Series, 203(2):18.

Witzel, G., Martinez, G., Hora, J., Willner, S. P., Morris, M. R., et al. (2018). Variability Timescale and Spectral Index of Sgr A* in the Near Infrared: Approximate Bayesian Computation Analysis of the Variability of the Closest Supermassive Black Hole. The Astrophysical Journal, 863(1):15.

Wollman, E. R., Geballe, T. R., Lacy, J. H., Townes, C. H., and Rank, D. M. (1977). Ne II 12.8 micron emission from the galactic center. II. ApJ, 218:L103-L107.

Wood, M., Caputo, R., Charles, E., Di Mauro, M., Magill, J., et al. (2017). Fermipy: An open-source Python package for analysis of Fermi-LAT Data. arXiv preprint arXiv:1707.09551.

Xu, Y.-D., Narayan, R., Quataert, E., Yuan, F., and Baganoff, F. K. (2006). Thermal X-Ray Iron Line Emission from the Galactic Center Black Hole Sagittarius A*. The Astrophysical Journal, 640(1):319-326.

Yang, H. Y., Ruszkowski, M., and Zweibel, E. (2018). Unveiling the Origin of the Fermi Bubbles. Galaxies, 6(1):29.

Yuan, F. and Narayan, R. (2014). Hot Accretion Flows Around Black Holes. ARA\&A, $52: 529-588$.

Yuan, F., Quataert, E., and Narayan, R. (2003). Nonthermal Electrons in Radiatively Inefficient Accretion Flow Models of Sagittarius A*. ApJ, 598:301-312. 
Yuan, F., Quataert, E., and Narayan, R. (2004). On the Nature of the Variable Infrared Emission from Sagittarius A*. ApJ, 606:894-899.

Yusef-Zadeh, F., Bushouse, H., Dowell, C. D., Wardle, M., Roberts, D., et al. (2006). A Multiwavelength Study of Sgr A*: The Role of Near-IR Flares in Production of X-Ray, Soft $\gamma$-Ray, and Submillimeter Emission. The Astrophysical Journal, 644(1):198-213.

Yusef-Zadeh, F., Bushouse, H., Wardle, M., Heinke, C., Roberts, D. A., et al. (2009). Simultaneous Multi-Wavelength Observations of Sgr A* During 2007 April 1-11. ApJ, 706:348-375.

Yusef-Zadeh, F., Morris, M., Slee, O. B., and Nelson, G. J. (1986). Nonthermal Radio Emission from the Galactic Center Arc. ApJ, 310:689.

Yusef-Zadeh, F., Roberts, D., Wardle, M., Heinke, C. O., and Bower, G. C. (2006). Flaring Activity of Sagittarius A* at 43 and 22 GHz: Evidence for Expanding Hot Plasma. ApJ, 650:189-194.

Yusef-Zadeh, F., Wardle, M., Dodds-Eden, K., Heinke, C. O., Gillessen, S., et al. (2012). An Inverse Compton Scattering Origin of X-Ray Flares from Sgr A*. The Astronomical Journal, 144(1):1.

Yusef-Zadeh, F., Wardle, M., Heinke, C., Dowell, C. D., Roberts, D., et al. (2008). Simultaneous Chandra, CSO, and VLA Observations of Sgr A*: The Nature of Flaring Activity. The Astrophysical Journal, 682(1):361-372.

Zamaninasab, M., Eckart, A., Witzel, G., Dovciak, M., Karas, V., et al. (2010). Near infrared flares of Sagittarius A* - Importance of near infrared polarimetry. A\&A, 510:A3.

Zhang, S., Baganoff, F. K., Ponti, G., Neilsen, J., Tomsick, J. A., et al. (2017). Sagittarius High-energy X-Ray Flare Properties during NuStar Monitoring of the Galactic Center from 2012 to 2015. The Astrophysical Journal, 843(2):96.

Zhao, J.-H. and Goss, W. M. (1998). Radio Continuum Structure of IRS 13 and Proper Motions of Compact $\mathrm{H}[\mathrm{CSC}] \mathrm{ii}[/ \mathrm{CSC}]$ Components at the Galactic Center. The Astrophysical Journal, 499(2):L163-L167. 
Zhao, J.-H., Young, K. H., Herrnstein, R. M., Ho, P. T. P., Tsutsumi, T., et al. (2003). Variability of Sagittarius A*: Flares at 1 Millimeter. ApJ, 586:L29-L32.

Zucker, S., Alexander, T., Gillessen, S., Eisenhauer, F., and Genzel, R. (2006). Probing Post-Newtonian Physics near the Galactic Black Hole with Stellar Redshift Measurements. The Astrophysical Journal, 639(1):L21-L24. 
Appendix 

Appendix A

\section{Article: Fermi LAT observations of Sagittarius A*: I - Imaging Analysis}

Here we reproduce the draft version of an Article as submitted in August $19^{\text {th }} 2020$ to The Astrophysical Journal which describe a significant part of the work presented in this Thesis. The $\mathrm{PhD}$ candidate is the first author and leader of the research presented in the document. 
Fermi LAT observations of Sagittarius A*: I - Imaging Analysis

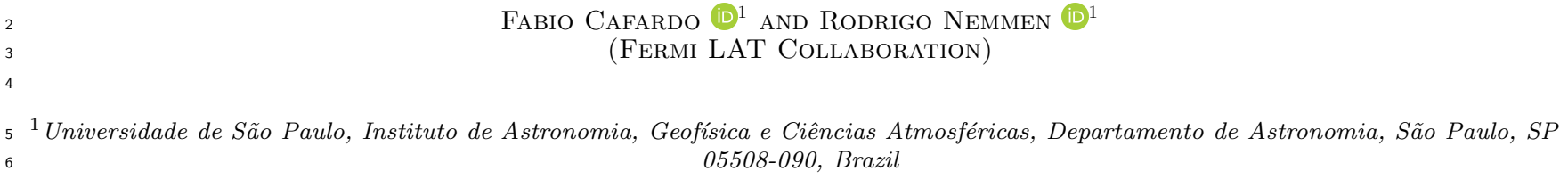

(Received XXXX; Revised XXXX; Accepted XXXX)

Submitted to ApJ

8

26 The center of our galaxy hosts a SMBH with a mass of ${ }_{27} \sim 10^{6} M_{\odot}$ (Ghez et al. 2008; Genzel et al. 2010; Boehle 28 et al. 2016) located at a distance of $8.2 \mathrm{kpc}$ (Abuter 29 et al. 2019). Like the Milky Way, it is believed that ev30 ery sufficiently massive galaxy harbors a SMBH in its 31 center (Lynden-Bell 1969; Kormendy \& Richstone 1995; 32 Miyoshi et al. 1995; Heckman \& Best 2014). The first 33 observations of a source that would later be associated 34 with Sgr $\mathrm{A}^{*}$ - the SMBH in our GC-were made by Bal35 ick \& Brown (1974) in radio wavelengths. Almost fifty 36 years later, there are several observations that confirm 37 the presence of the SMBH. The most convincing are 38 those obtained by Eisenhauer et al. (2005) and Gillessen 39 et al. (2009) monitoring stellar orbits and the detection

Corresponding author: Fabio Cafardo fabio.cafardo@usp.br
40 of orbital motions of a "hot spot" in the accretion flow 41 near the last stable circular orbit of Sgr A* (Gravity ${ }_{42}$ Collaboration et al. 2018). Results of the Event Hori43 zon Telescope observational campaign to reveal the im44 age of the shadow of Sgr $\mathrm{A}^{*}$ on the accretion flow are 45 still anxiously anticipated (e.g. Event Horizon Telescope ${ }_{46}$ Collaboration et al. 2019).

${ }_{47}$ The electromagnetic radiation from Sgr A* has been 48 seen in several wavelengths (e.g. Genzel et al. 2010; Mor49 ris et al. 2012; Eckart et al. 2018). It is highly variable 50 in the infrared (Genzel et al. 2003; Ghez et al. 2004; 51 Hornstein et al. 2007; Dodds-Eden et al. 2009; Witzel 52 et al. 2012; Hora et al. 2014; Witzel et al. 2018; von Fel53 lenberg et al. 2018; Fazio et al. 2018; Boyce et al. 2018) 54 and X-rays (Baganoff et al. 2001; Nowak et al. 2012; 55 Neilsen et al. 2013; Barrière et al. 2014; Neilsen et al. 56 2015; Ponti et al. 2015; Fazio et al. 2018; Boyce et al. 57 2018), which suggests a compact source. Variability has 58 also been reported in longer wavelengths (Zhao et al. 
59 2003; Miyazaki et al. 2004; Mauerhan et al. 2005; Mac60 quart et al. 2006; Yusef-Zadeh et al. 2006; Marrone et al. 61 2008; Yusef-Zadeh et al. 2009; Plambeck et al. 2014; ${ }_{62}$ Brinkerink et al. 2015; Stone et al. 2016). The $\gamma$-ray 63 flux, though, does not seem to be variable (Chernyakova 64 et al. 2011; Malyshev et al. 2015; Ahnen et al. 2017) and 65 there is still no definitive association between this emis66 sion and Sgr A*.

67 Following the H.E.S.S. TeV detections of the GC, sev68 eral candidates have been proposed for this $\gamma$-ray flux:

${ }_{69} \mathrm{Sgr} \mathrm{A}^{*}$ itself - either from its immediate vicinity (Aha-

70 ronian \& Neronov 2005a) or from a "plerion" produced

71 by the SMBH winds (Atoyan \& Dermer 2004; Kusunose

72 \& Takahara 2012) - ; the interaction between the dense 73 molecular clouds in the $\mathrm{GC}$ with cosmic rays acceler74 ated by Sgr $\mathrm{A}^{*}$ and/or by some other nearby source 75 (Aharonian \& Neronov 2005b; Ballantyne et al. 2011; 76 Chernyakova et al. 2011; Linden et al. 2012; Fatuzzo 77 \& Melia 2012; Guo et al. 2013); the pulsar wind neb78 ula (PWN) G359.95-0.04 (Wang et al. 2006; Hinton \& 79 Aharonian 2007); the supernova remnant Sagittarius A 80 East (Crocker et al. 2005) (but see Aharonian et al. 81 2009; Acero et al. 2010); the magnetar SGR J1745-2900 82 (Kennea et al. 2013; Mori et al. 2013) orbiting Sgr A*; 83 self-annihilating dark matter particles accumulating at 84 the GC (Hooper \& Goodenough 2011; Hooper \& Linden 85 2011) and an as-yet undetected pulsar (or population of 86 pulsars) (Hooper \& Linden 2011).

${ }_{87}$ It is believed that the quiescent state of $\mathrm{Sgr} \mathrm{A} \mathrm{A}^{*}$, ob88 served from radio to X-rays, is due to a radiatively inef89 ficient accretion flow (RIAF) (e.g. Narayan et al. 1995; 90 Yuan \& Narayan 2014). The broadband spectrum is 91 dominated by the radio-to-submm emission which is un92 derstood as synchrotron radiation from a thermal pop93 ulation of electrons, with temperatures between $\sim 5-20$ ${ }_{94} \mathrm{MeV}$, as well as a small fraction (a few percent) of non95 thermal electrons (Yuan et al. 2003). In its steady state, 96 Sgr A* emits $\sim 10^{36} \mathrm{erg} \mathrm{s}^{-1}$ (e.g. Genzel et al. 2010). ${ }_{97}$ The X-ray flare emission has been interpreted as Inverse ${ }_{98}$ Compton (IC) upscattered photons by the mildly rela99 tivistic, nonthermal electrons (Yusef-Zadeh et al. 2009; 100 Ball et al. 2016). Alternatively, X-ray flare emission has 101 been argued to be due to synchrotron emission (Dodds102 Eden et al. 2009).

103 Prominent gamma-ray emission from $\mathrm{MeV}$ to $\mathrm{TeV}$ en104 ergies coincident with Sgr A*'s position is observed by 105 Fermi LAT. Since the beginning of Fermi's operations, a 106 point source has been observed coinciding with the posi107 tion of Sgr A*. This source was studied by Chernyakova 108 et al. (2011) with 25 months of Fermi observations. ${ }_{109}$ They found no temporal variability at $\mathrm{GeV}$ energies and 110 proposed a model in which the $\gamma$-ray emission in the in-
111 ner $10 \mathrm{pc}$ of the Galaxy arises from relativistic proton 112 interactions. Later, Malyshev et al. (2015) analyzed the 113 same source using 74 months of data and the Second ${ }_{114}$ Catalogue of Fermi LAT Sources (Nolan et al. 2012). ${ }_{115}$ They also found no variability in the flux, and consid116 ered the observed spectrum as consistent with IC scat117 tering of high-energy electrons.

${ }_{118}$ Ahnen et al. (2017) collected several models for the $119 \mathrm{MeV}$ to TeV emission from Sgr A*. The list includes lep120 tonic (Kusunose \& Takahara 2012), hadronic (Fatuzzo 121 \& Melia 2012; Linden et al. 2012; Ballantyne et al. 2011; ${ }_{122}$ Chernyakova et al. 2011) and hybrid (Guo et al. 2013) 123 models. We call the models in this list "Fermi-era", 124 since they were all constructed taking in consideration 125 Fermi-LAT's data.

${ }_{126}$ The TeV emission observed by H.E.S.S. indicates the ${ }_{127}$ presence of $\mathrm{PeV}$ protons within the central $10 \mathrm{pc}$ of the ${ }_{128}$ Galaxy (HESS Collaboration et al. 2016). They pro129 pose that a more active phase of Sgr A* in the past could 130 have accelerated this population of high-energy protons. 131 There is also tantalizing evidence for an enhanced level 132 of activity in the recent past of Sgr A* through the Fermi 133 Bubbles (Su et al. 2010) which should have formed 1-3 ${ }_{134}$ Myr ago and endured for 0.1-0.5 Myr (Guo \& Mathews 135 2012; Yang et al. 2018). The origin of the bubbles is 136 still debated and could be also due to a previous star137 burst in addition to the activity of the SMBH. Further 138 evidence for higher levels of activity in Sgr A* comes 139 from X-ray observations of circumnuclear clouds (Ponti 140 et al. 2010). Concretely, X-ray observations since the ${ }_{141} 1990$ s show rapid variations in the $6.4 \mathrm{keV}$ of $\mathrm{Fe} \mathrm{K} \alpha$ 142 line propagating through molecular clouds in the inner ${ }_{143}$ Galactic regions. These variations are likely the result of 144 a highly variable active phase of Sgr A* within the past 145 few hundred years, which is echoing through the clouds. ${ }_{146}$ Models indicate at least two luminous outbursts $(\sim 100$ 147 and 400 years ago) on few-year timescales during which 148 the luminosity of $\mathrm{Sgr} \mathrm{A}^{*}$ went up to at least $10^{39} \mathrm{erg}$ $149 \mathrm{~s}^{-1}$ (Ponti et al. 2010; Clavel et al. 2013). In summary, 150 it seems that $\mathrm{Sgr} \mathrm{A}^{*}$ was $10^{3}$ times more active within 151 the past few centuries compared to current levels.

The GC is the closest example of a galactic nucleus 153 and a compelling laboratory to investigate the physical 154 processes responsible for accelerating particles to $\mathrm{TeV}$ 155 and $\mathrm{PeV}$ energies. Of the several sources near the GC 156 in the Fourth catalog of Fermi LAT sources (4FGL, Ab157 dollahi et al. 2020), 4FGL J1745.6-2859 is the brightest 158 and the closest to $\mathrm{Sgr} \mathrm{A}^{*}$ at a distance of $\sim 0.01^{\circ}$. Here, 159 we report an imaging analysis for this point source. In 160 section 2, we describe the observations and data analysis 161 procedure. In section 3 we describe the results from the 162 imaging analysis for the point source in four different 
163 energy ranges between $60 \mathrm{MeV}-500 \mathrm{GeV}$. In section 4 164 we discuss the possible candidates that can explain the 165 observed $\gamma$-ray emission. Finally, in section 5 we sum166 marize our main findings. In a forthcoming paper, we 167 will describe a light curve and spectral analysis for this 168 point source, with the overall goal of understanding the 169 nature of the processes responsible for the high-energy 170 emission of this source.

\section{2 . OBSERVATIONS AND DATA ANALYSIS}

172 In this work, we divided the analysis into four en173 ergy bands: $60-300 \mathrm{MeV}, 300 \mathrm{MeV}-3 \mathrm{GeV}, 3-10 \mathrm{GeV}$ 174 and $10-500 \mathrm{GeV}$. The goals were to study the impact of 175 photon energy on the location of the source, to measure 176 4FGL J1745.6-2859's $\gamma$-ray emission in different parts 177 of the electromagnetic spectrum to allow comparisons 178 with predictions of several models that try to explain 179 the GC's $\gamma$-ray flux (Section 4) and also to take ad180 vantage of the better Fermi-LAT point-spread function ${ }_{181}$ (PSF) at higher energies.

${ }_{182}$ The investigations of the three highest-energy bands 183 are based on an analysis (which we call "universal 184 model") performed with energies between $100 \mathrm{MeV}$ and $185500 \mathrm{GeV}$. The lowest-energy band used a custom model.

${ }_{186}$ Figure 1 shows how we split the analysis into different 187 energy bands and models.

${ }_{188}$ In this Section we show how the universal model 189 (and its descendants) and the low-energy custom model 190 were created and how they were used to assess 4FGL 191 J1745.6-2859's $\gamma$-ray flux and position in different en192 ergies.

\section{$193 \quad 2.1$. The universal model and its descendants}

194 Here we describe the process for the creation of a spa$195 \mathrm{tial} / \mathrm{spectral}$ model with photons between $100 \mathrm{MeV}$ and $196500 \mathrm{GeV}$. This model was used to evaluate the $\gamma$-ray 197 photon and energy fluxes of the source. Also, it was 198 used as the basis for three different analyses considering 199 only photons with energies between $300 \mathrm{MeV}-3 \mathrm{GeV}$, 200 3-10 GeV and 10-500 GeV.

201 In this part of the work, we used $\sim 11.3$ years (from 2022008 August to 2019 December) of Fermi-LAT (Atwood 203 et al. 2009) data. We considered a region of $20^{\circ} \times 20^{\circ}$ 204 square centered on the point source 4FGL J1745.6-2859 205 coincident with $\mathrm{Sgr} \mathrm{A}^{*}$ and rotated $\sim 58^{\circ} .6$ to the East 206 in Galactic coordinates.

207 Data were binned to a pixel size of $0^{\circ} .08$. We chose 208 the recommended ${ }^{1}$ value of $\geqslant 90^{\circ}$ for the zenith angle 209 cut.

1 https://fermi.gsfc.nasa.gov/ssc/data/analysis/documentation/ Cicerone/Cicerone_Data_Exploration/Data_preparation.html

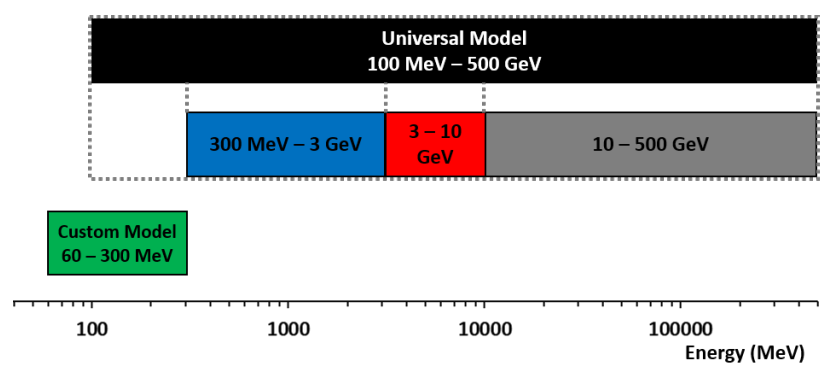

Figure 1. We divided our analyses into four energy bands (60-300 MeV, $300 \mathrm{MeV}-3 \mathrm{GeV}, 3-10 \mathrm{GeV}$ and $10-500 \mathrm{GeV})$. The models for the three highest-energy bands were created based on an analysis that considered energies in the range $100 \mathrm{MeV}-500 \mathrm{GeV}$. The lowest-energy band was studied with a custom model.

210 The region was modeled based on the sources' po211 sitions and spectral models in the preliminary re212 lease of 4FGL (gll_psc_v20.fit, Abdollahi et al. 213 2020), the updated model of interstellar $\gamma$-ray emission, 214 gll_iem_v07.fits, and standard isotropic spectral tem215 plates selected according to the event types and event 216 class used in this work. We performed a binned like217 lihood analysis using Fermitools conda package version 218 1.2.1, Fermipy python package version 0.17.4 (Wood 219 et al. 2017) and Pass 8 release 3 Version 2 response 220 functions (Atwood et al. 2013). Energy dispersion was 221 disabled for the isotropic diffuse component only.

The Fermi-LAT PSF varies considerably with photon 223 energy. It ranges from $\gtrsim 10^{\circ}(68 \%$ containment for pho224 tons with event class SOURCE) for photons with energies ${ }_{225} \gtrsim 100 \mathrm{MeV}$ to $\lesssim 1^{\circ}$ for $\gtrsim 10 \mathrm{GeV}$ photons. In this work, 226 we use photons classified as SOURCE.

Fermi-LAT photons are classified in different event 228 types. They can be categorized according to the location 229 where they converted in the detector (photons converted 230 in the front of the equipment have better-measured di231 rections than those converted in the back) or according 232 to the quality of the reconstructed direction (the pho233 tons are divided in quartiles depending on the quality of 234 this reconstruction). In this work we chose to consider 235 only the $75 \%$ photons with the best-reconstructed direc236 tions (event types PSF1, PSF2 and PSF3). We performed ${ }_{237}$ a joint likelihood analysis with three components ac${ }_{238}$ counting for the isotropic emission because event types 239 PSF1, PSF2 and PSF3 have different isotropic spectral 240 templates.

To perform a likelihood analysis with Fermi-LAT 242 data, it is necessary to create a model of the $\gamma$-ray emit243 ting sources in the region of interest $(\mathrm{RoI})$. To generate 244 the model, we included the 4FGL sources inside a square 245 larger than the size of the RoI (with $25^{\circ}$ side) to account 246 for Fermi-LAT's PSF. We started by creating the uni- 
247 versal model with energies between $100 \mathrm{MeV}$ and 500 $248 \mathrm{GeV}$.

249 We began the analysis using Fermipy's optimize 250 method. This tool fits the spectral models of all sources 251 within the RoI through an iterative strategy: it starts 252 by simultaneously fitting the normalization parameters 253 of the brightest sources, then individually fits the nor254 malization of every source not included in the first step, 255 and finishes by individually fitting the normalizations 256 and indexes of sources with the largest TS. After that we 257 started fitting the sources in the RoI. Since the GC re258 gion is very rich in sources - there are 196 4FGL sources 59 in the central $10^{\circ}$ of the Galaxy - we used an iterative 260 approach for fitting of the RoI, always taking care to 261 limit the number of free parameters to $\lesssim 15$ as recom262 mended by the optimizer's (MINUIT) manual. Several 263 iterations were performed in order to fit the brightest sources closest to 4FGL J1745.6-2859. In all iterations, 265 the normalization of our source of interest was allowed 266 to vary as well as the normalizations of the Galactic 267 diffuse emission model (galdiff) and of the isotropic 268 spectral template (isodiff). The normalizations of se269 lected sources were also iteratively freed based on their 270 proximity to the center of the RoI and their brightness in $271 \gamma$-rays, measured by the number of predicted photons in 272 each energy interval. We performed several iterations to 273 fit the desired sources. Only the best-quality fits (Qual274 ity: 3, Status: 0) were considered. Fits that did not 275 converge or with lower quality were disregarded. In this 276 case we would go back to the previous step of the fit277 ting procedure and continue from there with fewer free 278 sources.

279 The next step was to use the Fermipy function 280 find_sources. Twenty-nine new sources were found 281 with this tool in the energy range between $100 \mathrm{MeV}$ 282 and $500 \mathrm{GeV}$. We used power-law spectral models for 283 each of the new sources. This spectral model was se284 lected because it is typically adequate for relatively faint 285 sources, and in fact the majority of sources in 4FGL are 286 modeled with a power-law spectrum. Finding and char287 acterizing new sources is not a goal of this work. The 288 aim of this source-finding step was to improve the qual289 ity of the model. Some of the newly found sources are 290 likely spurious detection due to unmodeled background 291 emission. The new sources are listed in Appendix A.

292 In between these steps we evaluated the quality of our 293 fitting procedures by two approaches:

- Residual maps: built by subtracting the modeled counts from the data and searching for regions with significant residuals.
- Test Statistics (TS, Mattox et al. 1996) maps: searching for the presence of an additional source component in each spatial bin of the RoI.

Maps that show no regions with TS $\gtrsim 25$ and Residso1 uals $\gtrsim|4 \sigma|$ were considered well modeled. Regions with ${ }_{02}$ excesses above these levels were fitted again. In these 303 cases, the normalizations of sources close to excesses in 304 those maps were allowed to vary, together with the nor305 malizations of 4FGL J1745.6-2859 and of the diffuse 306 components. Usually, mainly in the case of negative 307 residuals, this additional round of fitting was enough to 308 lessen the excesses in the maps while also increasing the 309 likelihood of the model. In the case of regions with pos310 itive TS, some of the excesses were reduced only after 311 using the find_sources tool.

312 The last step was fitting the spectral indexes of the 313 central source. The approach was the same as described 314 above. We performed different iterations of fitting, al315 ways with the normalization and the indexes of 4FGL $316 \mathrm{~J} 1745.6-2859$ as free parameters together with other 317 free parameters that usually included the normalization 318 and the spectral indexes of nearby bright sources and 319 the Galactic and isotropic diffuse models. Only itera320 tions with the best fit quality were considered.

These steps led to the creation of the universal model 322 with photons between $100 \mathrm{MeV}$ and $500 \mathrm{GeV}$. Then, it 323 was used as an initial model for the analysis in three 324 energy bands (300 MeV-3 GeV, 3-10 GeV and 10-500 $325 \mathrm{GeV}$ ). In each energy band, the only sources that were 326 allowed to vary in a new round of fitting were $4 \mathrm{FGL}$ ${ }_{327} \mathrm{~J} 1745.6-2859$ (normalization and index) and the Galac328 tic and isotropic diffuse models (normalization only). 329 This "minimal fitting" was used with the objective of 330 keeping a similar model in the three energy bands.

331 To determine the centroid of the $\gamma$-ray emission of the ${ }_{332}$ central point source for each energy range and calculate 333 the likelihood of it being spatially extended, we used 334 Fermipy's extension method. In addition to finding the 335 location of the point source, it computes the likelihood 336 of the source being extended with respect to it being ${ }_{337}$ pointlike. Also, it gives the best-fit model for extension. ${ }_{338}$ We chose to use a 2D Gaussian as the putative extended 339 spatial model for the central source.

\section{$340 \quad 2.2$. The low-energy model}

341 Using the universal model as a starting point for a new 342 model at lower energies proved to be challenging. We ${ }_{343}$ could not obtain a good-quality model with the same 344 minimal fitting used to "split" the universal model in 3 345 energy bands (described at the end of Section 2.1) nor 346 with several additional rounds of fitting: in both cases 347 we ended up with models whose Residuals and TS maps 
348 showed many regions with excesses above the acceptable 349 levels. So we decided to use a model created specifically 350 for the $60-300 \mathrm{MeV}$ energy band. We already had this 351 model prepared from previous studies. As will be de352 scribed below, some features of this model's data are 353 different from the universal model's. This is not a seri354 ous issue because, in this work, we treat the results for 355 each energy band independently, avoiding comparisons 356 between each other.

357 We considered data inside a $30^{\circ} \times 30^{\circ}$ square also cen358 tered on the point source 4FGL J1745.6-2859 and with

359 the same orientation as the one used to the universal 360 model. This choice of size for the RoI is appropriate for 361 modeling the distribution of lower-energy photons given 362 the broad PSF. We used $0^{\circ} .1$ pixel size and the recom363 mended value of $\geqslant 90^{\circ}$ for the zenith angle cut. About 36410.5 years of Fermi LAT data was considered (from 2008 365 August to 2019 February).

${ }_{366}$ We modeled the region using the preliminary re367 lease of 4FGL (gll_psc_v17.fit, Abdollahi et al 368 2020), the updated model of interstellar $\gamma$-ray emission, 369 gll_iem_v07.fits, and standard isotropic spectral tem370 plates. We included in the model all 4FGL sources in a 371 region with $35^{\circ}$ side to account for Fermi-LAT's PSF.

372 Energy dispersion was disabled for the isotropic and 373 Galactic diffuse components.

$374 \quad$ As for the analysis described in Section 2.1 we used 375 event class SOURCE, event types PSF1, PSF2 and PSF3 and 376 performed a joint likelihood analysis with three compo377 nents accounting for the isotropic emission.

Before starting the analysis we changed the Spectrum 379 Type of 4FGL J1745.6-2859. It is catalogued as log380 parabola but we adopted a power-law in this low-energy 381 model. We used results obtained in previous analy382 sis using the Third Fermi LAT catalog (3FGL, Acero 383 et al. 2015) as the starting values for the parameters 384 that were later refitted with the new data. The main 385 reason for the change was the ease of fitting power-law 386 spectra, which have one less parameter compared to log387 parabolas. This change is appropriate since in narrow 388 energy bands (like the ones we are using here) a log389 parabola can be approximated by a power law.

${ }_{390}$ After this, we followed the same process described 391 in Section 2.1 to fit the model. During this pro392 cess, we found 14 new sources with Fermipy's func393 tion find_sources in regions associated with TS $>25$. 394 But in new rounds of fitting, several of them showed a 395 reduction of their TS to values below 25. Since Fermi396 LAT's PSF is broader at lower energies, we decided 397 to exclude one at a time - the new sources in this 398 condition from the model to avoid them interfering 399 in the results of our source of interest. We started
400 by excluding the farthest from the center of the RoI 401 and refitted the model. The normalizations of the 402 sources closest to the excluded one were left free, to403 gether with the normalizations of the Galactic diffuse 404 emission model, of the isotropic spectral template and 405 of 4FGL J1745.6-2859. We repeated this process until 406 there were no new sources with TS $<25$ in the model. 407 After excluding these sources, we ended up with 5 new 408 sources. They are listed in Appendix B. As we men409 tioned in Section 2.1, these new sources may be spurious 410 detections due to inaccuracies in the models. Finally, ${ }_{411}$ we used Fermipy's extension method to assess the lo${ }_{412}$ cation of 4FGL J1745.6-2859 in this energy range.

41

${ }_{414}$ This study has some similarities to the work of Maly${ }_{415}$ shev et al. (2015), with several improvements. The most ${ }_{416}$ obvious is the longer time baseline of the photon data417 more than 10.5 years of observations versus 6 years${ }_{418}$ which provides better statistics. This allows for sharper 419 images and better modelling with reduced source con${ }_{420}$ fusion. Also, we are using more-recent versions of Fer${ }_{421}$ mitools, Pass 8 response functions and the preliminary 422 version of 4FGL catalog (accompanied with improved ${ }_{423}$ versions of the diffuse models), all of which were released ${ }_{424}$ after their work. Finally, we chose to use a stricter event 425 type selection than Malyshev et al. (2015).

\section{RESULTS}

${ }_{427}$ In our work, we subdivided the analysis into four ${ }_{428}$ energy ranges. In Figure 2 we compare the results 429 of these four models with the log-parabola spectral 430 model adopted in the 4FGL Catalogue for 4FGL ${ }_{431} \mathrm{~J} 1745.6-2859$. We can see there is a considerable dis432 crepancy between the low $(60-300 \mathrm{MeV})$ and higher 433 energy $(>300 \mathrm{MeV})$ spectral models. This is the result 434 of the different modelling for the $60-300 \mathrm{MeV}$ band, ${ }_{435}$ as discussed in Section 2. Only part of this difference 436 can be explained by the addition in our $60-300 \mathrm{MeV}$ 437 model of a new source (not included in 4FGL but listed ${ }_{438}$ as PS J1750.6-2723 in Appendix B) at a distance $<2^{\circ}$ 439 from 4FGL J1745.6-2859. This difference means that ${ }_{440}$ the $\gamma$-ray flux for this energy band might be underesti${ }_{441}$ mated. It is important to notice that the GC is among 442 the most complicated regions in the sky to study with 443 Fermi data: in addition to the high density of sources, 444 the region is also engulfed by the Galactic diffuse emis${ }_{445}$ sion. These factors are enhanced in lower energies due 446 to the large PSF.

${ }_{447}$ The results of our fitting steps discussed in Section ${ }_{448} 2$ can be seen in Figure 3. These residual maps are 449 useful to assess goodness-of-fit. The colors indicate the 


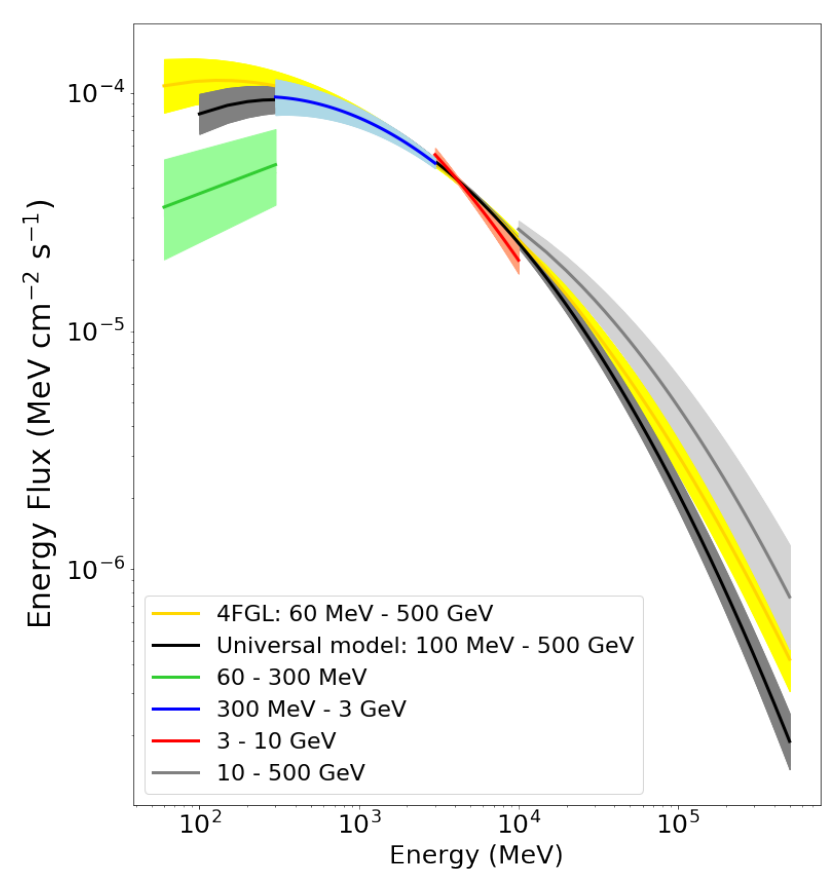

Figure 2. Comparison of spectral models for 4FGL J1745.6-2859. The black line shows the universal model created in the $100 \mathrm{MeV}-500 \mathrm{GeV}$ energy range. It was later used as basis to create models in three different energy bands: $300 \mathrm{MeV}-3 \mathrm{GeV}$ (blue), 3-10 GeV (red) and 10-500 GeV (gray). They were created with log-parabolas templates. A power-law model was used in the $60-300 \mathrm{MeV}$ energy range (green). The log-parabola spectral model used by the 4FGL Catalogue for this source is also shown (yellow).

${ }_{450}$ significance $^{2}(\sigma)$ of the residual (calculated as the dif${ }_{451}$ ference between the data and the model) in each en452 ergy band used in this work. Positive residuals indicate 453 regions that are underpredicted whereas negative ones 454 indicate overpredicted regions. There are some regions 455 with $|\sigma|>5$ in the models with energies $>300 \mathrm{MeV}$, ${ }_{456}$ especially in the $300 \mathrm{MeV}-3 \mathrm{GeV}$ energy band. They ${ }_{457}$ are the result of the minimal fitting we did when split${ }_{458}$ ting the analysis in the different energy bands. This pre459 vented us from getting better models but provided mod460 els that are comparable between the 3 highest-energy 461 bands. To assess the impact of this modelling on the 462 results, we created unique models from scratch for each 463 energy band and performed the same analysis. The re${ }_{464}$ sults we got were compatible in $1 \sigma$ with the ones pre465 sented within this work both in terms of the $\gamma$-ray fluxes 466 and the position of the source.

\footnotetext{
2 For a description on how $\sigma$ is calculated: https://fermipy.readthedocs.io/en/latest/advanced/residmap.html
}

${ }_{467}$ The four panels in Figure 4 show the TS map for each ${ }_{468}$ energy range. They were constructed using the tsmap 469 tool. This tool moves a putative point source through 470 the RoI and performs a maximum likelihood fit at each 471 point. We used a power-law spectral model with a spec472 tral index of -2 (with $d N / d E \propto E^{\alpha}$ where $d N / d E$ is 473 the differential photon flux and $\alpha$ is the spectral index). ${ }_{474}$ In the maps, the source position in each energy range is 475 marked by a colored circle in the center of the images. 476 The central point source itself is not visible in the maps 477 since it is part of the model. For the $60-300 \mathrm{MeV}$ range 478 there is no region with $\mathrm{TS} \geq 25$ (i.e. no emission con479 sistent with a point source with a power-law spectrum 480 with index -2 has significance $\gtrsim 5 \sigma$ ). This shows that 481 the models include all significant sources in this field. ${ }_{482}$ But in the maps of the high-energy $(>300 \mathrm{MeV})$ mod${ }_{483}$ els this is not true. These models were created with 484 minimal fitting based on the universal model (Section 4852.1 ) which led to regions that could be better modeled 486 if its sources were refitted. Nevertheless, the results we ${ }_{487}$ got for these energy models are compatible within $1 \sigma$ 488 with the ones obtained through models created specifi489 cally for each energy band (and that had no residual TS $490>25)$.

491 The central source was detected in the four energy 492 ranges used in the analysis with TS varying from $\approx 300$ ${ }_{493}$ to $\approx 10000$, corresponding to detections with signifi494 cance above background ranging from $\approx 17 \sigma$ to $\approx 100 \sigma$. 495 Its photon and energy flux were also measured and the 496 results are shown in Table 1.

${ }_{497}$ Assuming a distance to 4FGL J1745.6-2859 of $8.2 \mathrm{kpc}$ 498 (Abuter et al. 2019) and isotropic emission, the energy 499 flux of $(3.26 \pm 0.05) \times 10^{-10} \mathrm{erg} \mathrm{cm}^{-2} \mathrm{~s}^{-1}$ measured in 500 the $100 \mathrm{MeV}$ to $500 \mathrm{GeV}$ energy range corresponds to 501 a $\gamma$-ray luminosity of $(2.61 \pm 0.05) \times 10^{36} \mathrm{erg} \mathrm{s}^{-1}$. This 502 luminosity is comparable to the observed radio-to-X-ray ${ }_{503}$ luminosity of Sgr $\mathrm{A}^{*} \sim 10^{36} \mathrm{erg} \mathrm{s}^{-1}$ (Genzel et al. 2010). ${ }_{504}$ The four panels of Figure 5 show the TS maps of the 505 inner $8^{\circ} \times 8^{\circ}$ of the RoI, showing the presence of the 506 central point source. They were constructed using the 507 tsmap tool (again considering a putative point with a 508 spectral index of -2 ) and excluding the source 4FGL 509 J1745.6-2859 from the models. TS excesses are ob510 served in every panel and are always coincident with 511 4FGL J1745.6-2859's position. The apparent size of 512 the excess in the TS maps seems to decrease with en513 ergy, but this does not correspond to any variation of 514 the physical extension of the source. It is, instead, an 515 outcome of the improvement of Fermi LAT's PSF with 516 energy, as mentioned in Section 2.

517 The same diagnostic plots shown in Figures 3, 4 and 5185 for the different energy bands were used to evaluate 

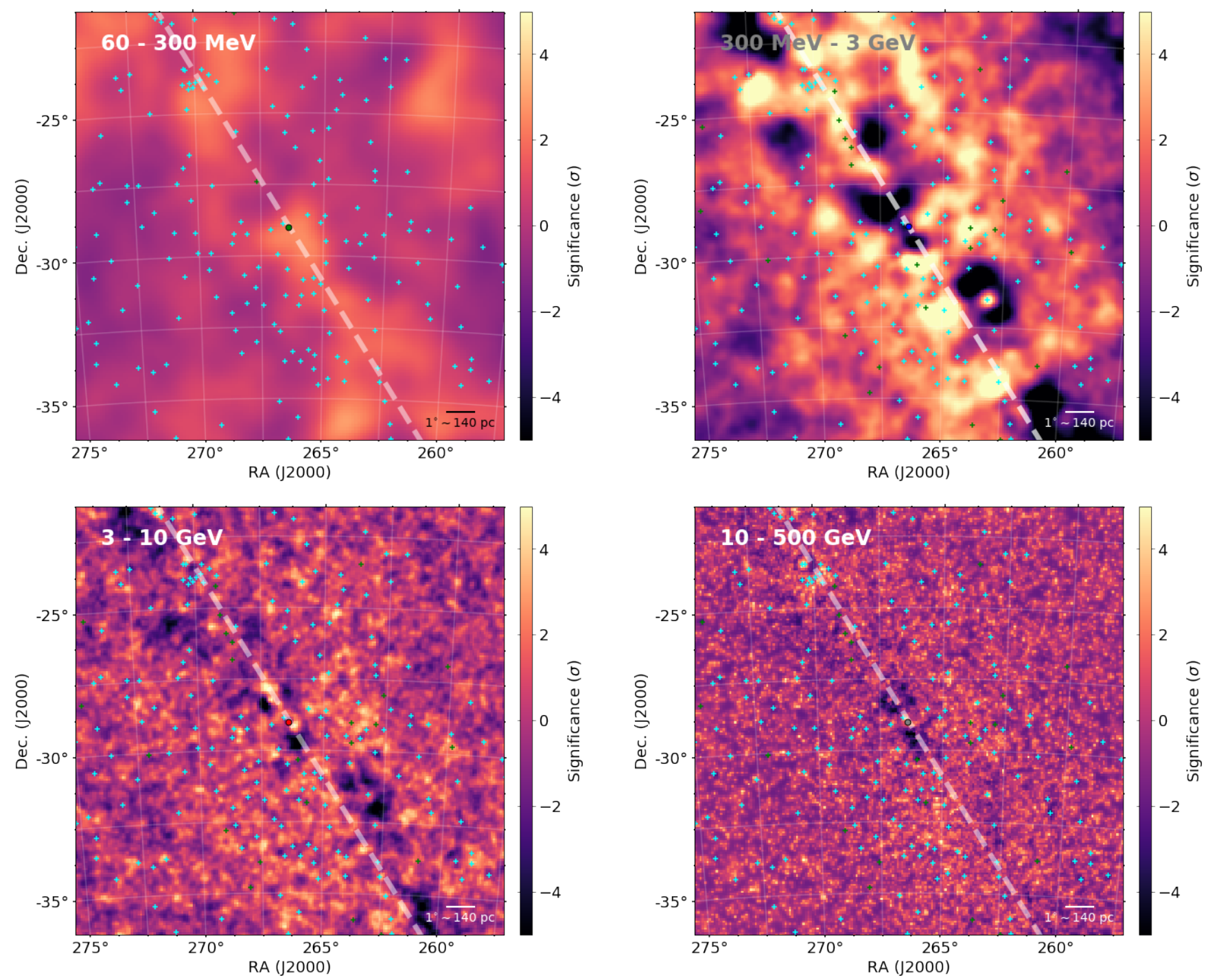

Figure 3. Residual maps for the four different energy ranges. The colors show the significance of the residual. The point at the center of each panel corresponds to the central source position obtained in each energy range. 4FGL point sources are displayed as cyan crosses and new sources found during the analysis as green crosses. The gray dashed lines indicate the direction of the Galactic equator. An angular separation of $1^{\circ}$ corresponds to $\sim 140 \mathrm{pc}$ at Sgr A*'s distance (8.2 kpc).

519 the quality of the fit of the universal model. They are 520 shown in Appendix C.

${ }_{521}$ We used the localize tool to constrain the point 522 source location in each energy range. Figure 6 shows ${ }_{523}$ the dependence of the source location on energy, to${ }_{524}$ gether with the radio position of $\mathrm{Sgr} \mathrm{A}^{*}$ as measured 525 by the Very Long Baseline Array (Petrov et al. 2011) ${ }_{526}$ and locations of other potential $\gamma$-ray emitters in the $527 \mathrm{GC}$.

${ }_{528}$ To calculate the total errors $\Delta_{t o t}$ on the location of 529 the source we followed the approach used by Abdollahi 530 et al. (2020) for the creation of the 4FGL Catalog:

$$
\Delta_{\text {tot }}^{2}=\left(f_{\text {rel }} \Delta_{\text {stat }}\right)^{2}+\Delta_{\text {abs }}^{2}
$$

${ }_{531}$ For the absolute precision $\Delta_{a b s}$ we used the value of $5320 .^{\circ} 0068$ (Abdollahi et al. 2020) in the two energy bands 533 below $3 \mathrm{GeV}$ and $0 .^{\circ} 0075$ (Ajello et al. 2017) for higher534 energy bands. For the systematic factor $f_{r e l}$ we used 5351.1 for the three lower-energy bands and 1.2 for the ${ }_{536} 10-500 \mathrm{GeV}$ band, these are conservative values based 537 on Fermi-LAT's reported PSF systematic uncertainty ${ }^{3}$.

\footnotetext{
${ }^{3}$ https://fermi.gsfc.nasa.gov/ssc/data/analysis/ LAT_caveats.html
} 

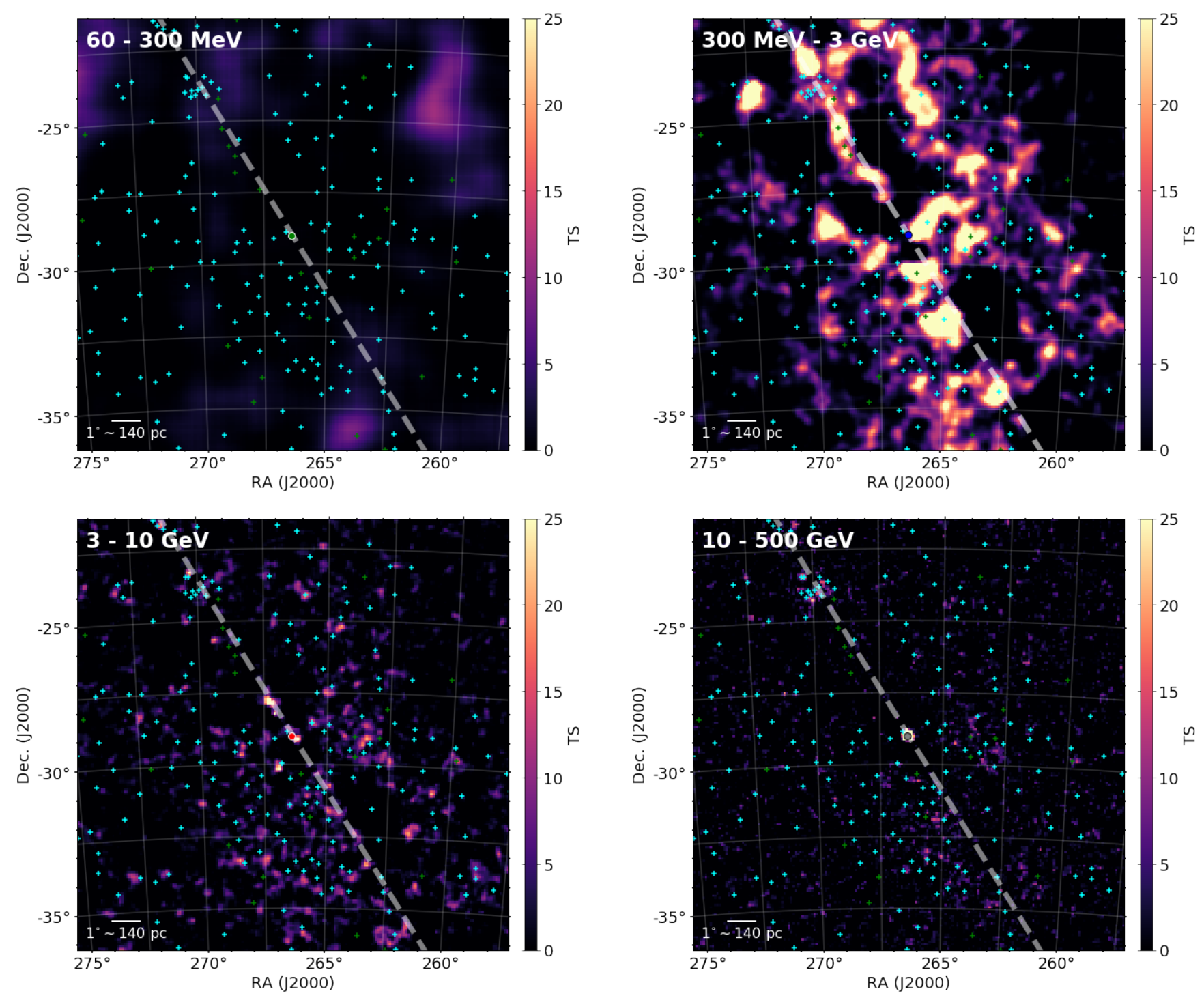

Figure 4. TS maps of the RoI for the four different energy ranges. The central point source is not prominent in these maps since it is part of the model. The circles at the center of the panels correspond to the central point source position obtained in each energy range. 4FGL point sources are displayed as cyan crosses and new sources found during the analysis as green crosses. The gray dashed lines indicate the direction of the Galactic equator. An angular separation of $1^{\circ}$ corresponds to $\sim 140$ pc at $\operatorname{Sgr} \mathrm{A}^{*}$ 's distance $(8.2 \mathrm{kpc})$.

${ }_{538}$ The statistical errors $\Delta_{\text {stat }}$ are the $68 \%$ positional un539 certainties obtained reported by the localize tool.

${ }_{540}$ In Figure 6 we can see that the position uncertainty of 541 the source in the lowest energy range is the largest. This ${ }_{542}$ is the result of a combination of factors: the broadening ${ }_{543}$ of Fermi LAT's PSF at lower energies, the energy de544 pendence of the instrument's field of view and effective 545 area, the central source's spectrum and the impact of 546 the Galactic diffuse emission which is more prominent 547 at lower energies. The position of the source is consis548 tent within $1 \sigma$ with $\mathrm{Sgr} \mathrm{A}^{*}$ in the energy ranges $60-300$ ${ }_{549} \mathrm{MeV}$ and $10-500 \mathrm{GeV}$. The $\gamma$-centroid recedes from Sgr
${ }_{550} \mathrm{~A}^{*}$ as the energy decreases. This is also seen in Figure 7, 551 which shows the distance between 4FGL J1745.6-2859 ${ }_{552}$ and $\mathrm{Sgr} \mathrm{A}^{*}$ as a function of energy.

553 We also tested the likelihood of the source being ex554 tended versus a point source using the extension tool. ${ }_{555}$ Figure 8 shows the $95 \%$ upper limits on the spatial ex556 tension of the source for each energy range. We find no 557 conclusive evidence for any spatially extended emission 558 from the central source.

\section{DISCUSSION}




\begin{tabular}{ccccccc}
\hline \hline $\begin{array}{c}\text { Energy } \\
\text { range }(\mathrm{GeV})\end{array}$ & TS $^{1}$ & $\begin{array}{c}\text { Photon flux } \\
\left(\mathrm{cm}^{-2} \mathrm{~s}^{-1}\right)\end{array}$ & $\begin{array}{c}\text { Energy flux } \\
\left(\mathrm{erg} \mathrm{cm}^{-2} \mathrm{~s}^{-1}\right)\end{array}$ & $\begin{array}{c}\text { Centroid } \\
\left({ }^{\circ}\right)\end{array}$ & $\begin{array}{c}\text { Positional Uncertainty }^{3} \\
\text { statistical }\end{array}$ \\
\hline $0.06-0.3$ & 2246 & $\left.(5.17 \pm 0.16) \times 10^{-7}\right)$ & $(1.06 \pm 0.03) \times 10^{-10}$ & $266.407,-29.013$ & 0.045 & 0.050 \\
total $\left(^{\circ}\right)$
\end{tabular}

$1 \sqrt{T S} \approx$ detection significance of the source in each energy range

2 RA and Dec (in the J2000 epoch) corresponding to the emission centroid in degrees

$368 \%$ positional uncertainty

Table 1. Results from the likelihood modeling of central point source. The last line presents the results for the universal model. The photon and energy flux uncertainties are statistical only. 5612859 (i.e., that it is located at the GC) we obtain the in562 teresting result that its $\gamma$-ray luminosity is similar to $\mathrm{Sgr}$ ${ }_{563} \mathrm{~A}^{*}$ 's radio to X-ray luminosity, about $10^{36} \mathrm{erg} \mathrm{s}^{-1}$. The 564 strong similarity between the electromagnetic energetics 565 of 4FGL J1745.6-2859 and Sgr A*, combined with the 566 positional coincidence, naturally suggests that the $\gamma$-ray 567 point source investigated in this work is associated with 568 the accreting SMBH. Nevertheless, the $0.1^{\circ}-1^{\circ}$ PSF of ${ }_{569}$ Fermi LAT encompasses a region of size $\sim 10-100 \mathrm{pc}$ 570 around the GC. Even the more constraining limit of $\lesssim$ ${ }_{571} 0^{\circ} .24$ for the central source's extension upper limit (Fig572 ure 8) corresponds to $\lesssim 35 \mathrm{pc}$ at the distance of Sgr A*, 573 thus allowing for several other potential candidates for 574 the $\gamma$-ray production site. Here, we list the most promis575 ing ones and discuss their likelihood at accounting for 576 4FGL J1745.6-2859.

577 The SMBH: Aharonian \& Neronov (2005a) argue that 578 due to Sgr A*'s low bolometric luminosity compared to 579 other SBMHs, the $\gamma$-rays produced close to the event 580 horizon - or in the inner parts of the accretion flow${ }_{581}$ can escape the source and be detected by Fermi LAT 582 because the absorption through photon-photon pair pro583 duction is low. Aharonian \& Neronov (2005a) consid584 ered three scenarios for the $\mathrm{TeV}$ photons detected by 585 H.E.S.S., two being hadronic and one leptonic. The first 586 hadronic model considers emission related to accelerated 587 protons producing $\gamma$-rays through synchrotron and cur588 vature radiation. It predicts an energy flux lower than 589 a few $10^{-12} \mathrm{erg} \mathrm{cm}^{-2} \mathrm{~s}^{-1}$ in the energy range studied 590 in this work, below the values we observed. The second 591 hadronic scenario considers lower energy protons accel592 erated by the electric field close to the event horizon ${ }_{593}$ or by shocks in the accretion disks. Some parametriza594 tions of this model predict very peaked spectral energy 595 distributions (SEDs) in the energy ranges used in this ${ }_{596}$ work. Since these SEDs are very narrow, the energy 597 fluxes they predict are consistent only with the obser598 vations in one of the four energy ranges we used in this
599 work. Their leptonic model, in its turn, also fails to ex600 plain Fermi-LAT's observation of 4FGL J1745.6-2859: 601 its SED shows $E^{2} d N / d E \approx 4 \times 10^{-9} \mathrm{erg} \mathrm{cm}^{-2} \mathrm{~s}^{-1}$ at ${ }_{602} \approx 1 \mathrm{GeV}$, thus overpredicting our observed energy flux 603 around this energy.

${ }_{604}$ A "plerion" produced by electrons in Sgr A*'s winds: 605 Atoyan \& Dermer (2004) propose a model for Sgr A* in 606 which the quiescent radio and the flaring NIR and X-ray 607 emissions are generated by synchrotron radiation from ${ }_{608}$ the RIAF. The wind from the RIAF, in a process sim609 ilar to pulsar-powered plerions, generates the quiescent ${ }_{610} \mathrm{X}$-ray and $\mathrm{TeV}$ emissions at the wind termination shock ${ }_{611}$ at about $3 \times 10^{16} \mathrm{~cm}$ from the SMBH. Although it can ${ }_{612}$ explain H.E.S.S.' TeV observations, their model is not ${ }_{613}$ sufficient to explain the $\mathrm{MeV}-\mathrm{GeV}$ reported in this work. ${ }_{614}$ Even if we consider Sagittarius A West bremsstrahlung ${ }_{615}$ emission and the emission from a larger plerion (inflated ${ }_{616}$ to pc scales), which are prominent in the energy range 617 used in this work (e.g., their Figure 1), the energy flux ${ }_{618}$ we detected is still about one order of magnitude higher. ${ }_{619}$ On the other hand, Kusunose \& Takahara (2012) used 62025 months of Fermi LAT's data for the GC (reported by ${ }_{621}$ Chernyakova et al. 2011) and proposed a similar leptonic ${ }_{622}$ model in which electrons escaping from the vicinity of ${ }_{623} \mathrm{Sgr} \mathrm{A^{* }}$ accumulate in a region with a size of $10^{18} \mathrm{~cm}$ ${ }_{624}$ where the $\gamma$-rays are produced by IC scattering of soft 625 photons emitted by stars and dust around the GC. Im${ }_{626}$ portantly, they obtain energy fluxes similar $\left(\sim 10^{-10} \mathrm{erg}\right.$ ${ }_{627} \mathrm{~cm}^{-2} \mathrm{~s}^{-1}$ ) to the values observed here.

${ }_{628}$ The interaction between the dense molecular clouds 629 with cosmic rays: As an explanation for the $\gamma$-ray emis${ }_{630}$ sion from the GC, Aharonian \& Neronov (2005b) pre${ }_{631}$ sented a model of proton-proton interactions between ${ }_{632}$ the protons accelerated near the SMBH and the dense ${ }_{633}$ gas in the central $10 \mathrm{pc}$ of the Galaxy which are fol${ }_{634}$ lowed by $\pi^{0}$ decay to $\gamma$-rays. Aharonian \& Neronov's ${ }_{635}$ work was published before the beginning of operations ${ }_{636}$ of Fermi LAT. Their results are inconsistent with our 637 observations. 

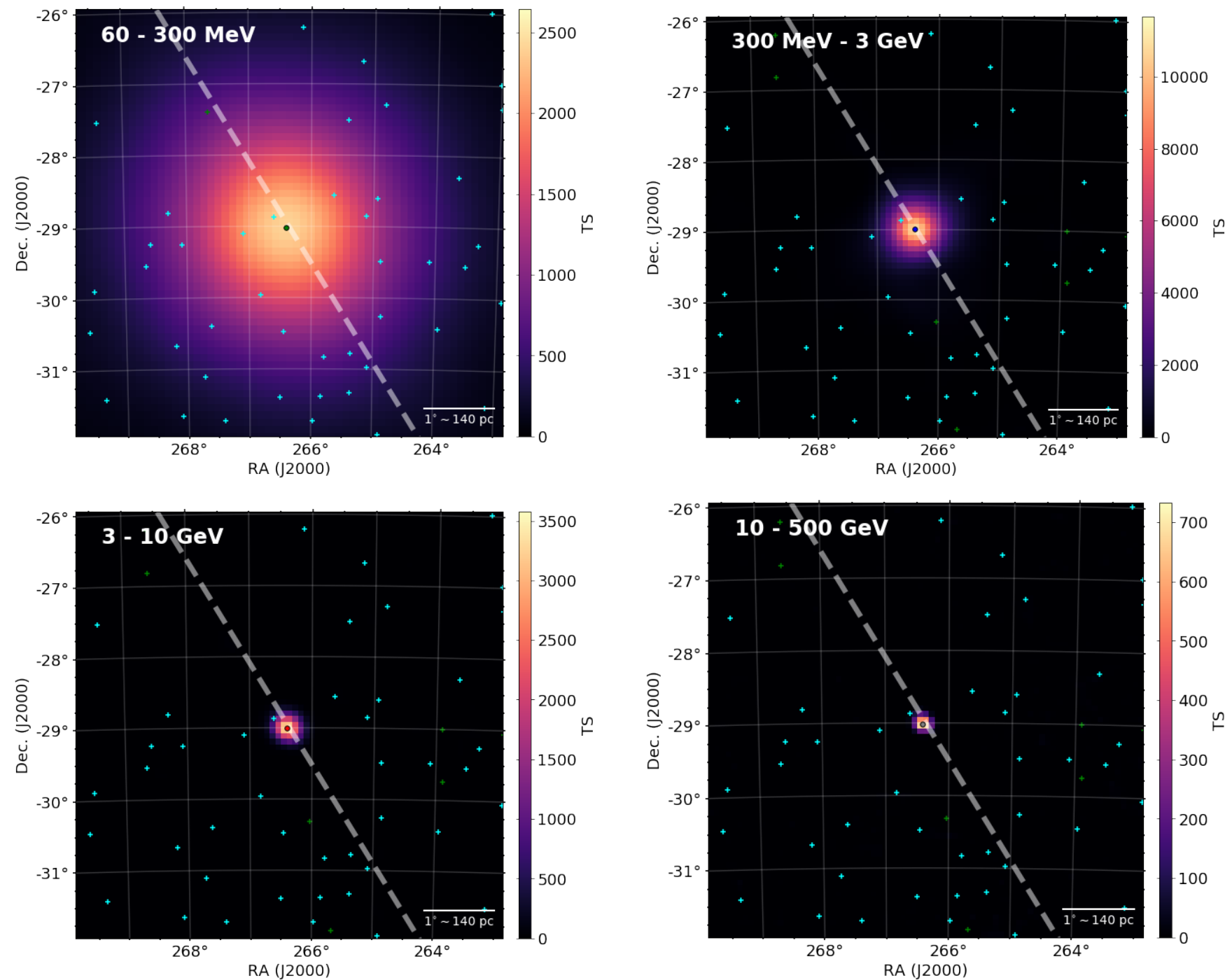

Figure 5. TS maps of the inner $8^{\circ} \times 8^{\circ}$ of the RoI showing the contribution of the central point source. They were constructed after excluding 4FGL J1745.6-2859 from the models, as explained in the text. The point at the center of each panel corresponds to the source position obtained in each energy range. The other 4FGL point sources are shown as cyan crosses and new sources found during the analysis are show as green crosses. The apparent sizes of the excesses decrease with energy, but this does not mean any variation of the physical extension of the source. Instead, it is an outcome of the broadening of Fermi LAT's PSF in low energies. The gray dashed lines indicate the direction of the Galactic equator. An angular separation of $1^{\circ}$ corresponds to $\sim 140 \mathrm{pc}$ at Sgr $\mathrm{A}^{*}$ 's distance $(8.2 \mathrm{kpc})$.

${ }_{638}$ More recent models take into account Fermi-LAT ob639 servations. For instance, Chernyakova et al. (2011) use 640 the first 25 months of Fermi-LAT and H.E.S.S. data 641 to create a hadronic model in which relativistic protons ${ }_{642}$ (presumably accelerated near Sgr $\mathrm{A}^{*}$ ) interact with the 643 gas in the inner parsecs of the Galaxy. Linden et al. ${ }_{644}$ (2012) developed a similar model. Another hadronic 645 model is proposed by Fatuzzo \& Melia (2012) where ${ }_{646}$ they consider a two-phase environment surrounding Sgr ${ }_{647} \mathrm{~A}^{*}$ : an inner high-density "torus" and the surround648 ing interstellar medium filled with shocked stellar winds
${ }_{649}$ which they call the "wind zone". Fermi $\gamma$-rays would be 650 produced in the "torus" and the higher energies would 651 come mostly from the "wind zone". Guo et al. (2013) ${ }_{652}$ propose a hybrid model. In their scenario, protons and ${ }_{653}$ electrons are accelerated in the GC (possibly around Sgr ${ }_{654} \mathrm{~A}^{*}$ ). Collisions between the protons and the interstel${ }_{655}$ lar gas would produce the $\mathrm{TeV} \gamma$-rays and the electrons ${ }_{656}$ would IC scatter the soft background photons.

${ }_{657}$ The four "Fermi-era" models mentioned above658 namely, Chernyakova et al. 2011; Linden et al. 2012; 659 Fatuzzo \& Melia 2012; Guo et al. 2013-are consistent 


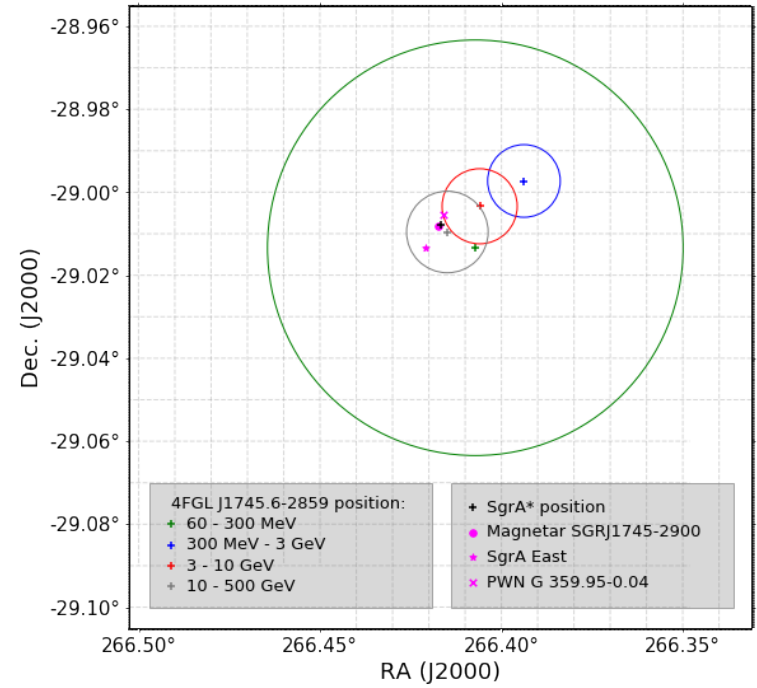

Figure 6. The position of the central source as a function of the energy range used in the analysis: green (100-300 MeV), blue $(300 \mathrm{MeV}-3 \mathrm{GeV})$, red $(3-10 \mathrm{GeV})$ and gray $(10-500$ $\mathrm{GeV})$. The circles represent the $68 \%$ positional uncertainty. The radio position of $\mathrm{Sgr} \mathrm{A}^{*}$ is indicated by the black cross The positions of other $\gamma$-ray-emitters in the GC are also indicated.

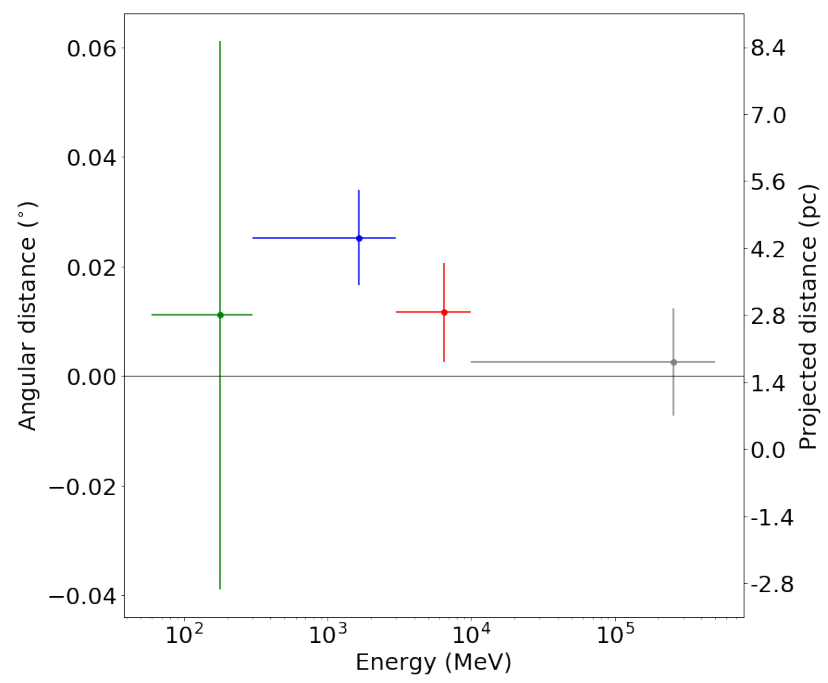

Figure 7. The distance between the central source and Sgr $\mathrm{A}^{*}$ as a function of energy.

660 with our observations, except in the lower energy band ${ }_{661}$ we used. This energy band is the one most subject ${ }_{662}$ to source confusion and to the impacts of the Galactic ${ }_{663}$ diffuse emission. This could explain why we observe ${ }_{664}$ greater energy fluxes in the $60-300 \mathrm{MeV}$ energy band ${ }_{665}$ than the prediction of these models: in addition to the ${ }_{666} \gamma$-rays created by the interaction between cosmic rays ${ }_{667}$ originated by (or nearby) Sgr A*, there is also a contri-

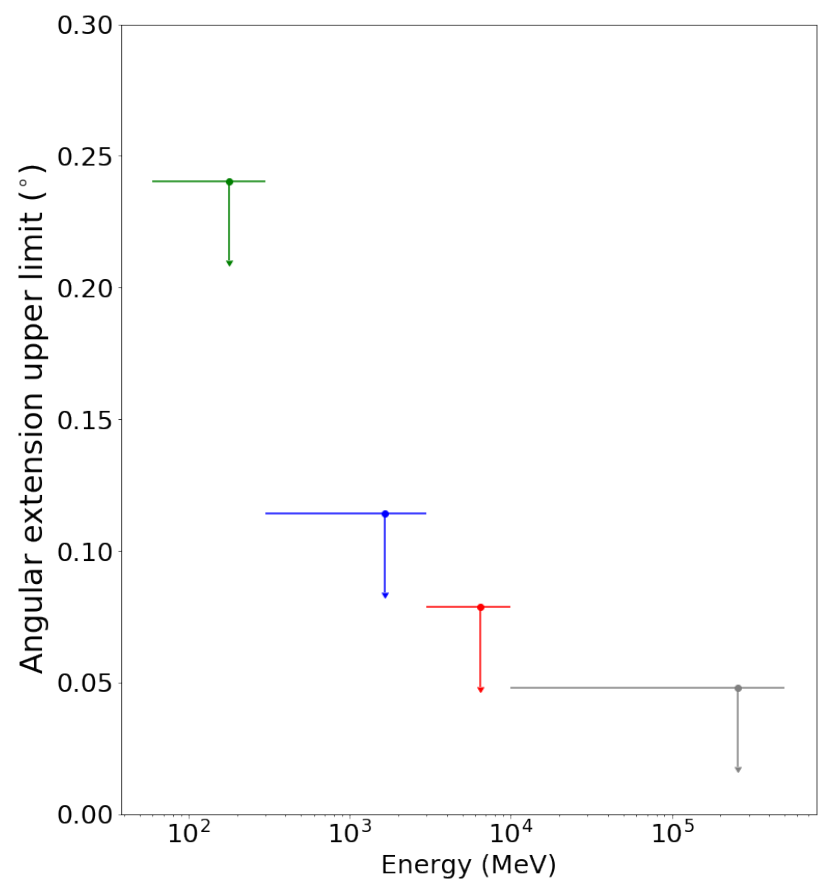

Figure 8. Upper limit on the spatial extension of 4FGL J1745.6-2859 as a function of energy. Upper limits correspond to the $95 \%$ confidence level.

668 bution from other sources. When Ahnen et al. (2017) 669 compared Fermi LAT's data reported by Malyshev et al. 670 (2015) with the "Fermi-era" models, the lower energy 671 data $(\lesssim 200 \mathrm{MeV})$ also showed greater fluxes than the 672 models' predictions.

${ }_{673}$ The PWN G 359.95-0.04: This X-ray nebula was dis674 covered by Wang et al. (2006) with a projected dis675 tance of only $0.32 \mathrm{pc}$ from Sgr $\mathrm{A}^{*}$ and was proposed 676 as an explanation for the $\mathrm{TeV}$ emission observed in the ${ }_{677}$ GC. Hinton \& Aharonian (2007) constructed theoreti678 cal SEDs for this source based on Chandra's detection 679 and supposing that the $\mathrm{TeV}$ emission of the H.E.S.S. 680 source HESS J1745-290 is from the PWN. Their mod681 els (e.g., their Figure 4) predicts energy fluxes on the ${ }_{682}$ order of $\sim 10^{-12} \mathrm{erg} \mathrm{cm}^{-2} \mathrm{~s}^{-1}$ for the energy ranges 683 studied in this work. This is more than one order of 684 magnitude lower than the energy fluxes we measured for ${ }_{685}$ 4FGL J1745.6-2859. This indicates that G 359.95-0.04 ${ }_{686}$ is not a good candidate to explain 4FGL J1745.6-2859's 687 emission.

${ }_{688}$ The supernova remnant Sagittarius A East: Sagit689 tarius A East is usually explained as a supernova rem690 nant, although other interpretations have also been sug${ }_{691}$ gested (Yusef-Zadeh \& Morris 1987; Mezger et al. 1989; ${ }_{692}$ Khokhlov \& Melia 1996). It is located in the inner par${ }_{693}$ secs of the Galaxy. Crocker et al. (2005) proposed it as 694 the source of the $\mathrm{TeV} \gamma$-rays from the GC. On the other 
695 hand, Aharonian et al. (2009) and Acero et al. (2010) 696 ruled out this association based on H.E.S.S. observations ${ }_{697}$ that show the origin of this emission as significantly dis698 placed from the position of Sagittarius A East. Posi699 tions of the central source found for the energy bands $700300 \mathrm{MeV}-3 \mathrm{GeV}$ and $3-10 \mathrm{GeV}$ suggest the same con701 clusion, as shown in Figure 6. But that is not the case 702 for the lowest and the highest energy bands (whose po703 sitions are coincident with Sagittarius A East). So we 704 can not completely rule out the association of the central 705 source with Sagittarius A East, although the connection 706 appears weak.

707 The magnetar SGR J1745-2900: This object was first 708 detected during a flare in 2013 with Swift's X-Ray Tele709 scope (Kennea et al. 2013) and NuSTAR (Mori et al. 710 2013). 4FGL J1745.6-2859's $\gamma$-ray light curve shows ${ }_{711}$ no sign of variability during this period (Malyshev et al. 712 2015; Ahnen et al. 2017; Cafardo et al., in preparation). 713 Also, it is predicted that the high-energy portion of the 714 spectra of magnetars peak at a few $\mathrm{MeV}$ (Thompson \& 715 Beloborodov 2005), while our work clearly shows emis716 sion from 4FGL J1745.6-2859 at energies > $10 \mathrm{GeV}$.

717 Self-annihilating dark matter particles accumulating 718 at the GC: Self-annihilating dark matter particles could 719 explain the GC $\gamma$-ray excess (Hooper \& Goodenough 720 2011; Hooper \& Linden 2011 and see Di Mauro 2020, 721 in preparation, for a recent analysis of the GC excess), 722 a surplus of $\sim \mathrm{GeV}$ diffuse emission that cannot be ex723 plained by the known catalogued sources. But is not a 724 satisfactory explanation for 4FGL J1745.6-2859 since 725 this is a point-like source rather than extended in space. 726 A population of pulsars surrounding the GC: The $\gamma$ 727 ray spectra of pulsars and millisecond pulsars can be 728 described as a power-law with an exponential cutoff 729 above a few GeV (Abdo et al. 2010). de Menezes et al. 730 (2019) studied the $\gamma$-ray emission of globular clusters in 731 the Milky Way - attributed to their large population of 732 millisecond pulsars - and found no significant flux above $733 \sim 10 \mathrm{GeV}$. In contrast with that, the point source 4FGL 734 J1745.6-2859 is detected at energies above that as indi735 cated in Table 1 and Figure 5. Its hard spectrum is also 736 not consistent with a pulsar (or population of them) in 737 the line of sight.

738

739 Except for the models constructed around Fermi 740 LAT's data, most of the candidates listed above are 741 unlikely to be solely responsible (if responsible at all) 742 for 4FGL J1745.6-2859's emission. It is possible that 743 some of them could explain, individually or together, 744 the lower energy emission where Fermi LAT's PSF is 745 broadest. One way to separate the contributions of 746 different candidates at lower energies is through model-
747 ing the MeV-to-GeV spectral energy distribution of the 748 point source. This is beyond the scope of the present 749 paper, and left for a forthcoming work.

750 From this work, we have the following main three re751 sults: (i) the energetics of 4FGL J1745.6-2859 is com752 parable to the radio-to-X-ray bolometric luminosity of 753 Sgr A*, (ii) among all the possible candidates for the $754 \gamma$-ray flux of the point source, only the models invoking 755 cosmic rays accelerated by Sgr A* or a nearby source can 756 explain the observations and (iii) the emission centroid 757 moves toward the position of Sgr A* as the energy is in758 creased. From these results, we conclude that $\mathrm{Sgr} \mathrm{A*}$ is 759 the most likely candidate to explain 4FGL J1745.6-2859 760 and its properties.

761 If we consider only the three highest-energy ranges 762 used in this analysis, the centroid emission moves in the 763 direction of $\operatorname{Sgr} \mathrm{A}^{*}$ as the photon energy is increased ${ }_{764}$ (Figures 6 and 7). Assuming a distance of $8.2 \mathrm{kpc}$, the 765 projected distances to $\mathrm{Sgr} \mathrm{A}^{*}$ as a function of energy 766 varies from $3.6 \pm 1.3 \mathrm{pc}(300 \mathrm{MeV}-3 \mathrm{GeV})$ to $0.4 \pm 1.4$ 767 pc $(10-500 \mathrm{GeV})$. This suggests that the particle popu768 lations responsible for the $\gamma$-rays detected in the three 769 bands are accelerated by the same process, originating 770 in the surroundings of $\mathrm{Sgr} \mathrm{A}^{*}$. The location centroid for 771 the lower-energy band is also consistent within $1 \sigma$ with 772 Sgr A*'s.

${ }_{773}$ As discussed in Section 2, the impact of source con774 fusion on Fermi-LAT observations is greater at lower 775 energies due to the PSF broadening. The localization 776 uncertainties are considerably larger at low energies, as 777 well as the limit on the angular extension, which creates 778 the possibility that several other sources and processes 779 are contributing to the lower-energy flux.

${ }_{780}$ We performed extension analysis of the source in the 781 four energy bands used in this work. In Figure 8, we re${ }_{782}$ port the $95 \%$ confidence level upper limit for the spatial 783 extension of the source. Again, we observe the impact of 784 energy on the results: in lower energies the source tends 785 to be more spatially extended. This can be a result of 786 the degradation of Fermi's PSF and source confusion.

\section{SUMMARY}

${ }_{788} \mathrm{Sgr} \mathrm{A}^{*}$ - the accreting SMBH at the center of our 789 galaxy - has been observed in virtually every band of 790 the electromagnetic spectrum. In $\gamma$-rays, H.E.S.S. and 791 Fermi LAT detected point sources coincident with Sgr ${ }_{792} \mathrm{~A}^{*}$. Nevertheless the connection between these point 793 sources and Sgr A* remained inconclusive. In this work 794 we have used about 10.5 years of Fermi LAT observa795 tions of the point source 4FGL J1745.6-2859 at the 796 Galactic Center with the aim of constraining the na797 ture of its emission. We divided the analysis into four 
798 different energy bands between $60 \mathrm{MeV}$ and $500 \mathrm{GeV}$, 799 performing a detailed imaging analysis of the surround800 ings of the point source. Our main conclusions can be 801 summarized as follows:

(i) The $0.1-500 \mathrm{GeV}$ luminosity of the point source 803 is $(2.61 \pm 0.05) \times 10^{36} \mathrm{erg} \mathrm{s}^{-1}$ assuming it is located 804 at the Galactic Center; this value is comparable to the 805 observed luminosity of Sgr A* from radio to X-rays.

806 (ii) The point source location approaches Sgr A*'s po807 sition as the photon energy is increased. For instance, 808 at energies $>10 \mathrm{GeV}$ the source location is consistent 809 with $\operatorname{Sgr} \mathrm{A}^{*}$ within $1 \sigma$.

${ }_{810}$ (iii) Among several possible candidates to the $\gamma$-ray 811 flux of the point source, only models invoking cosmic 812 rays - either hadronic or leptonic - accelerated by Sgr ${ }_{813} \mathrm{~A}^{*}$ or a nearby source can explain our observations.

${ }_{814}$ (v) Other processes not associated with the SMBH 815 could be contributing to the flux at energies $<300 \mathrm{MeV}$ ${ }_{816}$ (e.g., pulsars) due to the larger positional uncertainty of 817 the source as the instrument PSF deteriorates at lower 818 energies.

819 Taken together, our results support the picture in 820 which the point source 4FGL J1745.6-2859 observed ${ }_{821}$ by Fermi LAT at the GC is the manifestation of Sgr A* 822 in the $\mathrm{MeV}$-to-GeV range.

823 The advent of the Cherenkov Telescope Array (CTA, ${ }_{824}$ Cherenkov Telescope Array Consortium et al. 2019) will 825 allow for a deep exposure of the GC in energies up 826 to $\sim 300 \mathrm{TeV}$. This will permit studies in spatial and 827 spectral details unavailable today, with arc-minute reso828 lution at energies above Fermi's operational range, po829 tentially enabling a firmer association between the very 830 high-energy point source in the GC with Sgr A* or other 831 nearby candidate. Correspondingly, proposed $\gamma$-ray 832 missions focusing on $\mathrm{MeV}$ bands such as the AMEGO 833 mission (McEnery et al. 2019) and e-ASTROGAM (de 834 Angelis et al. 2018) should improve the observational 835 sensitivity, helping to better constrain the properties of 836 the GC emission in the $60-300 \mathrm{MeV}$ energy band and 837 shed light on the contribution of Sgr A*.

838

839 In a forthcoming work, we will analyze the $\gamma$-ray vari840 ability and SED of 4FGL J1745.6-2859 in order to fur${ }_{841}$ ther constrain its physical origin.

842

843 We acknowledge useful discussions with John Hewitt, ${ }_{844}$ Raniere de Menezes, Teddy Cheung, Matthew Kerr, Ju845 lia C. Santos., Giacomo Principe Jeremy S. Perkins and 846 Seth Digel.

${ }_{847}$ This work was supported by CNPq (Conselho Na848 cional de Desenvolvimento Científico e Tecnológico) un-
849 der grant 142320/2016-1 and FAPESP (Fundação de 850 Amparo à Pesquisa do Estado de São Paulo) under grant 851 2017/01461-2.

852 The Fermi LAT Collaboration acknowledges gener853 ous ongoing support from a number of agencies and 854 institutes that have supported both the development 855 and the operation of the LAT as well as scientific data 856 analysis. These include the National Aeronautics and 857 Space Administration and the Department of Energy in 858 the United States, the Commissariat à l'Energie Atom${ }_{859}$ ique and the Centre National de la Recherche Scien860 tifique / Institut National de Physique Nucléaire et de ${ }_{861}$ Physique des Particules in France, the Agenzia Spaziale ${ }_{862}$ Italiana and the Istituto Nazionale di Fisica Nucleare in 863 Italy, the Ministry of Education, Culture, Sports, Sci${ }_{864}$ ence and Technology (MEXT), High Energy Accelerator ${ }_{865}$ Research Organization (KEK) and Japan Aerospace Ex866 ploration Agency (JAXA) in Japan, and the K. A. Wal${ }_{867}$ lenberg Foundation, the Swedish Research Council and 868 the Swedish National Space Board in Sweden.

${ }_{869}$ Additional support for science analysis during the 870 operations phase is gratefully acknowledged from the 871 Istituto Nazionale di Astrofisica in Italy and the Cen872 tre National d'Études Spatiales in France. This work 873 performed in part under DOE Contract DE-AC02$87476 \mathrm{SF} 00515$.

\section{${ }_{875}$ Facilities: Fermi LAT}

${ }_{876}$ Software: Fermipy (Wood et al. 2017), APLpy (Ro877 bitaille \& Bressert 2012; Robitaille 2019). 


\section{APPENDIX}

\section{A. NEW SOURCES FOUND IN THE ANALYSIS BETWEEN 100 MEV AND 500 GEV}

880 Here we present the new sources encountered in the RoI with Fermipy's find_sources function in the energy range 881 between $100 \mathrm{MeV}$ and $500 \mathrm{GeV}$. The maximum likelihood parameters for the power-law (with $d N / d E \propto N_{0} \times E^{\alpha}$, ${ }_{882}$ where $d N / d E$ is the differential photon flux, $N_{0}$ is the prefactor and $\alpha$ is the spectral index) used to model their 883 spectra are also shown, together with their TS and position.

${ }_{884}$ Some of these newly detected source might be spurious due to unmodeled or inadequately modeled background 885 emission.

\begin{tabular}{cccccc}
\hline \hline Source name & Index & Prefactor & TS & RA & Dec \\
\hline PS J1719.1-2945 & -5.0 & $2.8 \times 10^{-14}$ & 236 & 259.80 & -29.75 \\
PS J1720.6-2655 & -2.1 & $4.5 \times 10^{-13}$ & 40 & 260.16 & -26.93 \\
PS J1723.8-3347 & -2.0 & $5.8 \times 10^{-13}$ & 31 & 260.95 & -33.79 \\
PS J1729.5-3623 & -1.9 & $4.7 \times 10^{-13}$ & 40 & 262.39 & -36.40 \\
PS J1730.5-2801 & -2.0 & $3.8 \times 10^{-13}$ & 31 & 262.64 & -28.03 \\
PS J1731.6-2903 & -1.8 & $2.9 \times 10^{-13}$ & 34 & 262.92 & -29.05 \\
PS J1733.8-2114 & -2.0 & $2.8 \times 10^{-13}$ & 30 & 263.47 & -21.24 \\
PS J1734.4-3555 & -2.2 & $5.8 \times 10^{-13}$ & 34 & 263.62 & -35.93 \\
PS J1734.6-2328 & -2.2 & $4.9 \times 10^{-13}$ & 37 & 263.66 & -23.48 \\
PS J1735.5-2944 & -1.8 & $3.3 \times 10^{-13}$ & 31 & 263.88 & -29.74 \\
PS J1735.6-2900 & -5.0 & $1.6 \times 10^{-14}$ & 36 & 263.91 & -29.01 \\
PS J1742.7-3150 & -2.0 & $8.0 \times 10^{-13}$ & 50 & 265.68 & -31.85 \\
PS J1744.1-3019 & -2.2 & $2.7 \times 10^{-12}$ & 124 & 266.03 & -30.32 \\
PS J1747.2-2114 & -2.3 & $5.8 \times 10^{-13}$ & 40 & 266.81 & -21.25 \\
PS J1750.4-3355 & -2.2 & $5.6 \times 10^{-13}$ & 40 & 267.61 & -33.93 \\
PS J1752.0-3447 & -2.2 & $4.1 \times 10^{-13}$ & 34 & 268.02 & -34.79 \\
PS J1752.6-2105 & -5.0 & $4.0 \times 10^{-14}$ & 303 & 268.15 & -21.09 \\
PS J1754.4-2612 & -2.1 & $7.6 \times 10^{-13}$ & 32 & 268.60 & -26.21 \\
PS J1754.4-2649 & -2.0 & $5.8 \times 10^{-13}$ & 30 & 268.60 & -26.82 \\
PS J1754.7-3730 & -2.2 & $3.7 \times 10^{-13}$ & 38 & 268.70 & -37.51 \\
PS J1755.3-2553 & -2.0 & $9.3 \times 10^{-13}$ & 49 & 268.83 & -25.90 \\
PS J1756.0-3248 & -2.1 & $4.1 \times 10^{-13}$ & 31 & 269.02 & -32.81 \\
PS J1756.3-2515 & -2.3 & $2.1 \times 10^{-12}$ & 99 & 269.08 & -25.25 \\
PS J1756.8-2413 & -1.9 & $5.4 \times 10^{-13}$ & 36 & 269.21 & -24.22 \\
PS J1758.0-2421 & -2.2 & $1.1 \times 10^{-12}$ & 38 & 269.52 & -24.36 \\
PS J1808.2-3003 & -2.2 & $4.5 \times 10^{-13}$ & 45 & 272.06 & -30.05 \\
PS J1817.4-2516 & -2.4 & $5.7 \times 10^{-13}$ & 50 & 274.35 & -25.27 \\
PS J1818.6-2812 & -2.6 & $5.0 \times 10^{-13}$ & 57 & 274.65 & -28.22 \\
PS J1823.9-2341 & -2.4 & $5.1 \times 10^{-13}$ & 47 & 275.99 & -23.69 \\
\hline & & & & & \\
\hline
\end{tabular}

Table 2. New sources found in the $100 \mathrm{MeV}$ to $500 \mathrm{GeV}$ energy range. RA and Dec are in the J2000 epoch. 
36 B. NEW SOURCES FOUND IN THE ANALYSIS WITH THE CUSTOM MODEL BETWEEN 60 AND 300 MEV

${ }_{887}$ Five new sources were encountered in the RoI with Fermipy's find_sources function in the energy range between 88860 and $300 \mathrm{MeV}$. They are listed in Table 3. The best parameters for the power-law (with $d N / d E \propto N_{0} \times E^{\alpha}$, where ${ }_{889} d N / d E$ is the differential photon flux, $N_{0}$ is the prefactor and $\alpha$ is the spectral index) used to model their spectra are 890 also shown, together with their TS and position.

891 These might be spurious findings due to imperfections in the model.

\begin{tabular}{cccccc}
\hline \hline Source name & Index & Prefactor & TS & RA & Dec \\
\hline PS J1639.5-2448 & -1.7 & $4.4 \times 10^{-12}$ & 37 & 249.90 & -24.81 \\
PS J1750.6-2723 & -2.0 & $4.8 \times 10^{-12}$ & 27 & 267.65 & -27.40 \\
PS J1753.7-2127 & -0.9 & $2.9 \times 10^{-12}$ & 57 & 268.44 & -21.47 \\
PS J1820.5-2113 & -2.0 & $4.3 \times 10^{-12}$ & 61 & 275.14 & -21.22 \\
PS J1835.0-1804 & -5.0 & $8.8 \times 10^{-15}$ & 183 & 278.76 & -18.07 \\
\hline
\end{tabular}

Table 3. New sources found in the 60 to $300 \mathrm{MeV}$ energy range. RA and Dec are in the J2000 epoch. 


\section{DIAGNOSTIC PLOTS FOR THE UNIVERSAL MODEL}

893 We present the residual map, the TS maps with and without 4FGL J1745.6-2859 in the universal model created in 894 the $100 \mathrm{MeV}$ to $500 \mathrm{GeV}$ energy range.
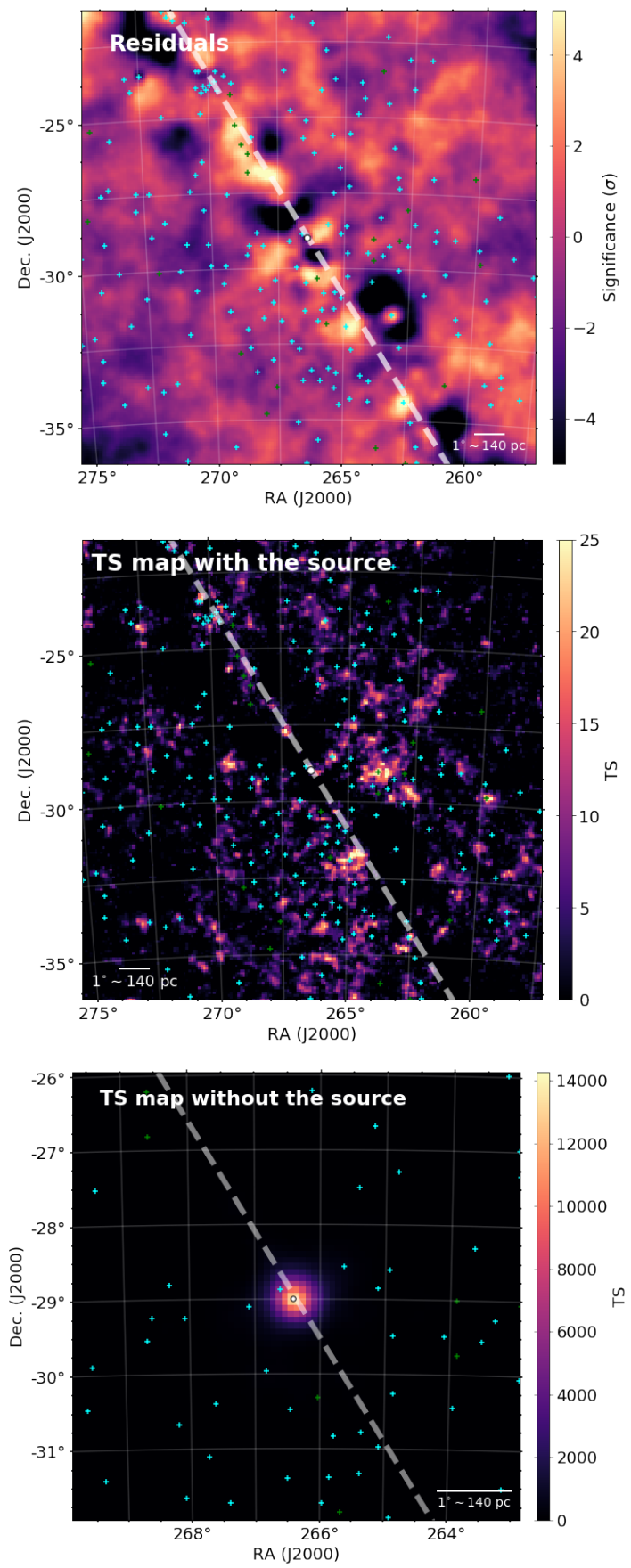

Figure 9. Diagnostic plots for the universal model. In the upper panel, the colors show the significance of the residual. On the other panels, the colors indicate the TS value in each position. The point at the center of each panel corresponds to 4FGL J1745.6-2859 position in the 4FGL Catalogue. 4FGL point sources are displayed as cyan crosses and new sources found during the analysis as green crosses. The gray dashed lines indicate the direction of the Galactic equator. An angular separation of $1^{\circ}$ corresponds to $\sim 140 \mathrm{pc}$ at $\mathrm{Sgr} \mathrm{A}^{*}$ 's distance $(8.2 \mathrm{kpc})$. 


\section{REFERENCES}

895 Abdo, A. A., Ackermann, M., Ajello, M., et al. 2010, ApJS,

896 187, 460, doi: 10.1088/0067-0049/187/2/460

897 Abdollahi, S., Acero, F., Ackermann, M., et al. 2020, The

898 Astrophysical Journal Supplement Series, 247, 33,

899 doi: $10.3847 / 1538-4365 / a b 6 b c b$

900 Abuter, R., Amorim, A., Bauboeck, M., et al. 2019, arXiv e-prints, arXiv:1904.05721.

https://arxiv.org/abs/1904.05721

903 Acero, F., Aharonian, F., Akhperjanian, A. G., et al. 2010,

904 MNRAS, 402, 1877,

905 doi: $10.1111 /$ j.1365-2966.2009.16014.x

906 Acero, F., Ackermann, M., Ajello, M., et al. 2015, ApJS,

907 218, 23, doi: 10.1088/0067-0049/218/2/23

908 Aharonian, F., \& Neronov, A. 2005a, ApJ, 619, 306,

909 doi: $10.1086 / 426426$

${ }_{910}$-. 2005b, Astrophysics and Space Science, 300, 255,

911 doi: $10.1007 /$ s10509-005-1209-4

912 Aharonian, F., Akhperjanian, A. G., Anton, G., et al. 2009

913 A\&A, 503, 817, doi: 10.1051/0004-6361/200811569

914 Ahnen, M. L., Ansoldi, S., Antonelli, L. A., et al. 2017,

${ }_{915}$ A\&A, 601, A33, doi: 10.1051/0004-6361/201629355

916 Ajello, M., Atwood, W. B., Baldini, L., et al. 2017, ApJS,

$917 \quad 232$, 18, doi: 10.3847/1538-4365/aa8221

918 Atoyan, A., \& Dermer, C. D. 2004, ApJL, 617, L123,

919 doi: $10.1086 / 427390$

920 Atwood, W., Albert, A., Baldini, L., et al. 2013, arXiv

921 preprint arXiv:1303.3514

922 Atwood, W. B., Abdo, A. A., Ackermann, M., et al. 2009,

${ }_{923}$ ApJ, 697, 1071, doi: 10.1088/0004-637X/697/2/1071

${ }_{924}$ Baganoff, F. K., Bautz, M. W., Brandt, W. N., et al. 2001,

925 Nature, 413, 45, doi: 10.1038/35092510

926 Balick, B., \& Brown, R. L. 1974, ApJ, 194, 265,

927 doi: $10.1086 / 153242$

${ }_{928}$ Ball, D., Özel, F., Psaltis, D., \& Chan, C.-k. 2016, ApJ,

$929 \quad 826,77$, doi: 10.3847/0004-637X/826/1/77

930 Ballantyne, D. R., Schumann, M., \& Ford, B. 2011,

931 Monthly Notices of the Royal Astronomical Society, 410,

932 1521, doi: 10.1111/j.1365-2966.2010.17533.x

933 Barrière, N. M., Tomsick, J. A., Baganoff, F. K., et al.

934 2014, The Astrophysical Journal, 786, 46,

935 doi: $10.1088 / 0004-637 x / 786 / 1 / 46$

936 Boehle, A., Ghez, A. M., Schödel, R., et al. 2016, ApJ, 830,

937 17, doi: 10.3847/0004-637X/830/1/17

938 Boyce, H., Haggard, D., Witzel, G., et al. 2018, arXiv

939 e-prints, arXiv:1812.05764.

940 https://arxiv.org/abs/1812.05764

941 Brinkerink, C. D., Falcke, H., Law, C. J., et al. 2015, A\&A,

$942 \quad$ 576, A41, doi: 10.1051/0004-6361/201424783
${ }_{943}$ Cherenkov Telescope Array Consortium, Acharya, B. S.,

944 Agudo, I., et al. 2019, Science with the Cherenkov

${ }_{945}$ Telescope Array, doi: 10.1142/10986

946 Chernyakova, M., Malyshev, D., Aharonian, F. A., Crocker,

${ }_{947}$ R. M., \& Jones, D. I. 2011, ApJ, 726, 60,

948 doi: $10.1088 / 0004-637 \mathrm{X} / 726 / 2 / 60$

949 Clavel, M., Terrier, R., Goldwurm, A., et al. 2013, A\&A,

$950 \quad$ 558, A32, doi: 10.1051/0004-6361/201321667

${ }_{951}$ Crocker, R. M., Fatuzzo, M., Jokipii, J. R., Melia, F., \&

952 Volkas, R. R. 2005, ApJ, 622, 892, doi: 10.1086/427972

953 de Angelis, A., Tatischeff, V., Grenier, I. A., et al. 2018,

954 Journal of High Energy Astrophysics, 19, 1,

955 doi: 10.1016/j.jheap.2018.07.001

956 de Menezes, R., Cafardo, F., \& Nemmen, R. 2019, Monthly

957 Notices of the Royal Astronomical Society, 486, 851,

958 doi: $10.1093 / \mathrm{mnras} / \mathrm{stz} 898$

959 Dodds-Eden, K., Porquet, D., Trap, G., et al. 2009, ApJ,

960 698, 676, doi: 10.1088/0004-637X/698/1/676

961 Eckart, A., Zajacek, M., Parsa, M., et al. 2018, arXiv

${ }_{962}$ e-prints, arXiv:1806.00284.

963 https://arxiv.org/abs/1806.00284

964 Eisenhauer, F., Genzel, R., Alexander, T., et al. 2005, The

965 Astrophysical Journal, 628, 246, doi: 10.1086/430667

966 Event Horizon Telescope Collaboration, Akiyama, K.,

967 Alberdi, A., et al. 2019, ApJ, 875, L1,

968 doi: $10.3847 / 2041-8213 /$ ab0ec7

969 Fatuzzo, M., \& Melia, F. 2012, ApJ, 757, L16,

970 doi: 10.1088/2041-8205/757/1/L16

971 Fazio, G. G., Hora, J. L., Witzel, G., et al. 2018, ApJ, 864,

972 58, doi: $10.3847 / 1538-4357 / \operatorname{aad} 4 \mathrm{a} 2$

973 Genzel, R., Eisenhauer, F., \& Gillessen, S. 2010, Reviews of

$974 \quad$ Modern Physics, 82, 3121,

975 doi: 10.1103/RevModPhys.82.3121

976 Genzel, R., Schödel, R., Ott, T., et al. 2003, Nature, 425,

977 934, doi: 10.1038/nature02065

978 Ghez, A. M., Wright, S. A., Matthews, K., et al. 2004,

979 ApJL, 601, L159, doi: 10.1086/382024

${ }_{980}$ Ghez, A. M., Salim, S., Weinberg, N. N., et al. 2008, ApJ,

$981 \quad 689,1044$, doi: 10.1086/592738

982 Gillessen, S., Eisenhauer, F., Trippe, S., et al. 2009, ApJ,

${ }_{983} 692,1075$, doi: 10.1088/0004-637X/692/2/1075

984 Gravity Collaboration, Abuter, R., Amorim, A., et al. 2018,

985 A\&A, 618, L10, doi: 10.1051/0004-6361/201834294

986 Guo, F., \& Mathews, W. G. 2012, ApJ, 756, 181,

987 doi: 10.1088/0004-637X/756/2/181

988 Guo, Y.-Q., Yuan, Q., Liu, C., \& Li, A.-F. 2013, Journal of

989 Physics G Nuclear Physics, 40, 065201,

990 doi: 10.1088/0954-3899/40/6/065201 
991 Heckman, T. M., \& Best, P. N. 2014, ARA\&A, 52, 589,

992 doi: 10.1146/annurev-astro-081913-035722

993 HESS Collaboration, Abramowski, A., Aharonian, F., et al.

994 2016, Nature, 531, 476, doi: 10.1038/nature17147

995 Hinton, J. A., \& Aharonian, F. A. 2007, ApJ, 657, 302,

996 doi: $10.1086 / 510283$

997 Hooper, D., \& Goodenough, L. 2011, Physics Letters B,

$998 \quad 697,412$, doi: 10.1016/j.physletb.2011.02.029

999 Hooper, D., \& Linden, T. 2011, PhRvD, 84, 123005 ,

1000 doi: 10.1103/PhysRevD.84.123005

1001 Hora, J. L., Witzel, G., Ashby, M. L. N., et al. 2014, The

1002 Astrophysical Journal, 793, 120,

1003 doi: $10.1088 / 0004-637 x / 793 / 2 / 120$

1004 Hornstein, S. D., Matthews, K., Ghez, A. M., et al. 2007,

1005 The Astrophysical Journal, 667, 900, doi: 10.1086/520762

1006 Kennea, J. A., Burrows, D. N., Kouveliotou, C., et al. 2013,

1007 The Astrophysical Journal, 770, L24,

1008 doi: 10.1088/2041-8205/770/2/124

1009 Khokhlov, A., \& Melia, F. 1996, ApJL, 457, L61,

1010 doi: $10.1086 / 309895$

1011 Kormendy, J., \& Richstone, D. 1995, ARA\&A, 33, 581,

1012 doi: 10.1146/annurev.aa.33.090195.003053

1013 Kusunose, M., \& Takahara, F. 2012, ApJ, 748, 34,

1014 doi: 10.1088/0004-637X/748/1/34

1015 Linden, T., Lovegrove, E., \& Profumo, S. 2012, ApJ, 753,

1016 41, doi: 10.1088/0004-637X/753/1/41

1017 Lynden-Bell, D. 1969, Nature, 223, 690,

1018 doi: $10.1038 / 223690 \mathrm{a0}$

1019 Macquart, J.-P., Bower, G. C., Wright, M. C. H., Backer,

1020 D. C., \& Falcke, H. 2006, The Astrophysical Journal,

1021 646, L111, doi: 10.1086/506932

1022 Malyshev, D., Chernyakova, M., Neronov, A., \& Walter, R.

1023 2015, A\&A, 582, A11, doi: 10.1051/0004-6361/201526120

1024 Marrone, D. P., Baganoff, F. K., Morris, M. R., et al. 2008,

1025 ApJ, 682, 373, doi: 10.1086/588806

1026 Mattox, J. R., Bertsch, D. L., Chiang, J., et al. 1996, ApJ,

1027 461, 396, doi: 10.1086/177068

1028 Mauerhan, J. C., Morris, M., Walter, F., \& Baganoff, F. K.

1029 2005, ApJL, 623, L25, doi: 10.1086/429960

1030 McEnery, J., van der Horst, A., Dominguez, A., et al. 2019,

1031 in Bulletin of the American Astronomical Society,

1032 Vol. 51, 245

1033 Mezger, P. G., Zylka, R., Salter, C. J., et al. 1989, A\&A,

$1034 \quad 209,337$

1035 Miyazaki, A., Tsutsumi, T., \& Tsuboi, M. 2004, The

1036 Astrophysical Journal, 611, L97, doi: 10.1086/424004

1037 Miyoshi, M., Moran, J., Herrnstein, J., et al. 1995, Nature,

$1038 \quad 373,127$, doi: $10.1038 / 373127 \mathrm{a} 0$
1039 Mori, K., Gotthelf, E. V., Zhang, S., et al. 2013, The

1040 Astrophysical Journal, 770, L23,

1041 doi: $10.1088 / 2041-8205 / 770 / 2 / 123$

1042 Morris, M. R., Meyer, L., \& Ghez, A. M. 2012, Research in

1043 Astronomy and Astrophysics, 12, 995,

1044 doi: 10.1088/1674-4527/12/8/007

1045 Narayan, R., Yi, I., \& Mahadevan, R. 1995, Nature, 374,

1046 623, doi: $10.1038 / 374623 \mathrm{aO}$

1047 Neilsen, J., Nowak, M. A., Gammie, C., et al. 2013, ApJ,

1048 774, 42, doi: 10.1088/0004-637X/774/1/42

1049 Neilsen, J., Markoff, S., Nowak, M. A., et al. 2015, ApJ,

1050 799, 199, doi: 10.1088/0004-637X/799/2/199

1051 Nolan, P. L., Abdo, A. A., Ackermann, M., et al. 2012,

1052 ApJS, 199, 31, doi: 10.1088/0067-0049/199/2/31

1053 Nowak, M. A., Neilsen, J., Markoff, S. B., et al. 2012, The

1054 Astrophysical Journal, 759, 95,

1055 doi: $10.1088 / 0004-637 x / 759 / 2 / 95$

1056 Petrov, L., Kovalev, Y. Y., Fomalont, E. B., \& Gordon, D.

1057 2011, AJ, 142, 35, doi: 10.1088/0004-6256/142/2/35

1058 Plambeck, R., Dexter, J., Bower, G. C., et al. 2014 ,

1059 Monthly Notices of the Royal Astronomical Society, 442,

1060 2797, doi: 10.1093/mnras/stu1039

1061 Ponti, G., Terrier, R., Goldwurm, A., Belanger, G., \& Trap,

1062 G. 2010, ApJ, 714, 732,

1063 doi: $10.1088 / 0004-637 \mathrm{X} / 714 / 1 / 732$

1064 Ponti, G., De Marco, B., Morris, M. R., et al. 2015,

1065 MNRAS, 454, 1525, doi: 10.1093/mnras/stv1537

1066 Robitaille, T. 2019, APLpy v2.0: The Astronomical

1067 Plotting Library in Python, doi: 10.5281/zenodo.2567476.

1068 https://doi.org/10.5281/zenodo.2567476

1069 Robitaille, T., \& Bressert, E. 2012, APLpy: Astronomical

1070 Plotting Library in Python, Astrophysics Source Code

1071 Library. http://ascl.net/1208.017

1072 Stone, J. M., Marrone, D. P., Dowell, C. D., et al. 2016,

1073 The Astrophysical Journal, 825, 32,

1074 doi: $10.3847 / 0004-637 x / 825 / 1 / 32$

1075 Su, M., Slatyer, T. R., \& Finkbeiner, D. P. 2010, ApJ, 724,

1076 1044, doi: 10.1088/0004-637X/724/2/1044

1077 Thompson, C., \& Beloborodov, A. M. 2005, ApJ, 634, 565,

1078 doi: $10.1086 / 432245$

1079 von Fellenberg, S. D., Gillessen, S., Graciá-Carpio, J., et al.

1080 2018, The Astrophysical Journal, 862, 129,

1081 doi: $10.3847 / 1538-4357 /$ aacd4b

1082 Wang, Q. D., Lu, F. J., \& Gotthelf, E. V. 2006, MNRAS,

$1083 \quad 367,937$, doi: 10.1111/j.1365-2966.2006.09998.x

1084 Witzel, G., Eckart, A., Bremer, M., et al. 2012, The

1085 Astrophysical Journal Supplement Series, 203, 18,

1086 doi: 10.1088/0067-0049/203/2/18 
1087 Witzel, G., Martinez, G., Hora, J., et al. 2018, The

1088 Astrophysical Journal, 863, 15,

1089 doi: $10.3847 / 1538-4357 /$ aace 62

1090 Wood, M., Caputo, R., Charles, E., et al. 2017, arXiv

1091 preprint arXiv:1707.09551

1092 Yang, H. Y., Ruszkowski, M., \& Zweibel, E. 2018, Galaxies, 1093 6, 29, doi: 10.3390/galaxies6010029

1094 Yuan, F., \& Narayan, R. 2014, ARA\&A, 52, 529,
1096 Yuan, F., Quataert, E., \& Narayan, R. 2003, ApJ, 598, 301, 1097 doi: $10.1086 / 378716$

1098 Yusef-Zadeh, F., \& Morris, M. 1987, ApJ, 320, 545,

1099 doi: $10.1086 / 165572$

1100 Yusef-Zadeh, F., Roberts, D., Wardle, M., Heinke, C. O., \&

1101 Bower, G. C. 2006, ApJ, 650, 189, doi: 10.1086/506375

1102 Yusef-Zadeh, F., Bushouse, H., Wardle, M., et al. 2009,

1103 ApJ, 706, 348, doi: 10.1088/0004-637X/706/1/348

1104 Zhao, J.-H., Young, K. H., Herrnstein, R. M., et al. 2003,

1105 ApJL, 586, L29, doi: 10.1086/374581 
Appendix B

\section{Additional Publications}

Other than the publications already mentioned in Section 1.7, and whose results are the core of this work, there were additional achievements, in therms of publications, obtained during this $\mathrm{PhD}$.

The first one is Gutiérrez et al. (2020) (already mentioned in Section 1.3.3.3 and reproduced in the following pages), from which the doctoral candidate was a coauthor. The Article explains an unprecedented NIR flare from Sgr A* in May 2019 as the result of particle acceleration to nonthermal energies in the innermost parts of the accretion flow. It was published in the The Astrophysical Journal Letters in March 2020. The results of this work are not part of this thesis: it is only mentioned as part of the literature in Section 1.3.3.3 and in this Section for the sake of completeness.

A contribution with Dr. Raniere de Menezes, who recently completed his $\mathrm{PhD}$ at this Institute and presented these results in his thesis, was also an accomplishment of the PhD. In this work (de Menezes et al. 2019) the gamma-ray emission of every known globular cluster in the Milky Way was estimated with Fermi-LAT data. This emission is attributed to their populations of millisecond pulsars, which are efficient gamma-ray emitters, and can be used as a tool to explore the dynamical processes leading to binary system formation in these environments. Although this work is not immediately related to the subject of this thesis, the observational characteristics of the gamma-ray emission of millisecond pulsars were used in Section 4.1.1 to rule out the possibility that the observed emission of 4FGL J1745.6-2859 is from one of these objects (or population) in the line of sight. This work was published in the Monthly Notices of the Royal Astronomical Society in March 2019.

Finally, as a member of the Fermi-LAT Collaboration, the doctoral candidate made a minor contribution in Ajello et al. (2020). This work is also not directly related to the 
major theme of this thesis. It is a catalog of AGNs detected by Fermi-LAT, published in The Astrophysical Journal, in April 2020. The candidate contribution was part of a collaborative effort to identify the synchrotron peak in the SED of sources candidates to be part of this catalog. The identification was performed visually as a first step for a following stricter fitting of the fitting.

In the following pages, we reproduce the published version of an Article lead by M.Sc. Eduardo Gutiérrez, and with this $\mathrm{PhD}$ candidate as a coauthor, which was published in The Astrophysical Journal Letters in March 2020. 


\title{
A Nonthermal Bomb Explains the Near-infrared Superflare of Sgr $\mathbf{A}^{*}$
}

\author{
Eduardo M. Gutiérrez ${ }^{1}$ (D), Rodrigo Nemmen ${ }^{2}$ (D) , and Fabio Cafardo ${ }^{2}$ (i)
}

${ }^{1}$ Instituto Argentino de Radioastronomía (IAR, CCT La Plata, CONICET/CIC), C.C.5, (1984) Villa Elisa, Buenos Aires, Argentina; emgutierrez@iar.unlp.edu.ar

${ }^{2}$ Universidade de São Paulo, Instituto de Astronomia, Geofísica e Ciências Atmosféricas, Departamento de Astronomia, São Paulo, SP 05508-090, Brazil Received 2019 December 20; revised 2020 February 22; accepted 2020 February 24; published 2020 March 12

\begin{abstract}
The Galactic center supermassive black hole, Sgr A*, has experienced a strong, unprecedented flare in 2019 May when its near-infrared luminosity reached much brighter levels than ever measured. We argue that an explosive event of particle acceleration to nonthermal energies in the innermost parts of the accretion flow-a nonthermal bomb-explains the near-infrared light curve. We discuss potential mechanisms that could explain this event such as magnetic reconnection and relativistic turbulence acceleration. Multiwavelength monitoring of such superflares in radio, infrared, and X-rays should allow a concrete test of the nonthermal bomb model and put better constraints on the mechanism that triggered the bomb.
\end{abstract}

Unified Astronomy Thesaurus concepts: Galactic center (565); Black hole physics (159); Non-thermal radiation sources (1119); Accretion (14)

\section{Introduction}

At the center of the Milky Way lies Sagittarius A* $\left(\operatorname{Sgr} A^{*}\right)$, a supermassive black hole (SMBH) with a mass of $M=4 \times$ $10^{6} M_{\odot}$ located at a distance of $8.2 \mathrm{kpc}$ (Abuter et al. 2019). Given its proximity, Sgr A* presents one of the best laboratories for studying the physics of black hole $(\mathrm{BH})$ accretion flows (Falcke \& Markoff 2013). Sgr $A^{*}$ has been detected in most of the electromagnetic spectrum (e.g., Dibi et al. 2014). The extremely low accretion rate and low luminosity observed in its quiescent state $\left(L_{\text {bol }} \sim 10^{36} \mathrm{erg} \mathrm{s}^{-1} \sim 2 \times 10^{-9} L_{\text {Edd }}\right.$ where $L_{\text {Edd }}$ is the Eddington luminosity) implies that the accretion flow is in a radiatively inefficient accretion flow (RIAF) state (e.g., Yuan \& Narayan 2014).

On top of the quiescent emission, Sgr $\mathrm{A}^{*}$ also exhibits frequent flares in X-rays (e.g., Neilsen et al. 2013; Ponti et al. 2015) and near-infrared (NIR; e.g., Genzel et al. 2003; Boyce et al. 2018). About one X-ray flare is seen per day with a typical duration of a few tens of minutes (Neilsen et al. 2013). The brightest observed X-rays flares are $\sim 100$ times above the quiescent level (e.g., Nowak et al. 2012). The NIR flares are even more frequent. X-ray flares usually follow the NIR ones after a few tens of minutes, but there are multiple NIR flares without an X-ray counterpart (e.g., Eckart et al. 2006; YusefZadeh et al. 2012; Ponti et al. 2017; but see Fazio et al. 2018). Flares are also observed in millimeter and submillimeter wavelengths (e.g., Yusef-Zadeh et al. 2006; Stone et al. 2016). They last from hours to days with amplitudes of $\sim 25 \%$ of the quiescent level (Yusef-Zadeh et al. 2008; Fazio et al. 2018).

In 2019 May, Do et al. (2019) observed an unprecedented NIR flare from Sgr A*-hereafter the "superflare"-with the Keck telescope. The peak flux exceeded the maximum historical value by a factor of two and the light curve (LC) afterward showed a factor of 75 drop in flux over a $2 \mathrm{hr}$ time span. Do et al. (2019) suggested that an increase in the SMBH accretion rate $\dot{M}$ could be responsible for the superflare, possibly due to additional gas deposited by the passage of the G2 object in 2014 or a windy star such as S0-2 in 2018. Nevertheless, Ressler et al. (2018) argued that the effect of S02 on the RIAF structure should be negligible. This, combined with the fact that the S-star cluster has no known stars more massive than S0-2 close to Sgr A* spells trouble for the "windy star" scenario.

Here, we propose an entirely different scenario for the superflare that does not rely on an $\dot{M}$-increase: an explosive event of particle acceleration to nonthermal energies in the innermost parts of the accretion flow-a nonthermal bomb. This model explains quantitatively the NIR LC and makes testable predictions at other wavelengths.

\section{Model}

Our model for the emission involves an RIAF with populations of thermal and nonthermal electrons, following the height-integrated approach of Yuan et al. (2003). For simplicity, we assume that the dynamical structure of the flow (i.e., $\rho, v, T$ ) does not vary with time, but we consider the possibility that an unspecified acceleration mechanism may change the number of particles following a nonthermal energy distribution.

We take into account the presence of outflows by allowing the accretion rate to decrease with radius as $\dot{M}(r)=\dot{M}_{\max }\left(r / r_{\max }\right)^{s}$ (Blandford \& Begelman 1999), with $s=0.25$. We are only interested in the inner parts of the flow, so we only consider the accretion flow up to $r_{\max }=10^{3} r_{\mathrm{S}}$ where we set $\dot{M}_{\text {out }} \approx 10^{-7} M_{\odot} \mathrm{yr}^{-1}$. The other parameters are the fraction of turbulent energy directly transferred to electrons $\delta=0.33$, the viscosity parameter $\alpha=0.1$, and the gas pressure to magnetic pressure ratio $\beta=9$.

\subsection{Quiescent State}

To reproduce the quiescent state of the spectral energy distribution (SED), we assume that in each shell of the RIAF a fraction $\eta_{\mathrm{q}}=0.4 \%$ of the thermal energy density of electrons is in a nonthermal population with a broken power-law distribution:

$$
N_{\mathrm{q}}(\gamma ; r)=\left\{\begin{array}{ll}
K_{\mathrm{q}}(r) \gamma^{-p}, & \text { if } \gamma_{\min } \leqslant \gamma \leqslant \gamma_{\mathrm{c}}, \\
K_{\mathrm{q}}(r)(p-1) \gamma_{\mathrm{c}} \gamma^{-(p+1)}, & \text { if } \gamma_{\mathrm{c}} \leqslant \gamma \leqslant \gamma_{\max }
\end{array},\right.
$$




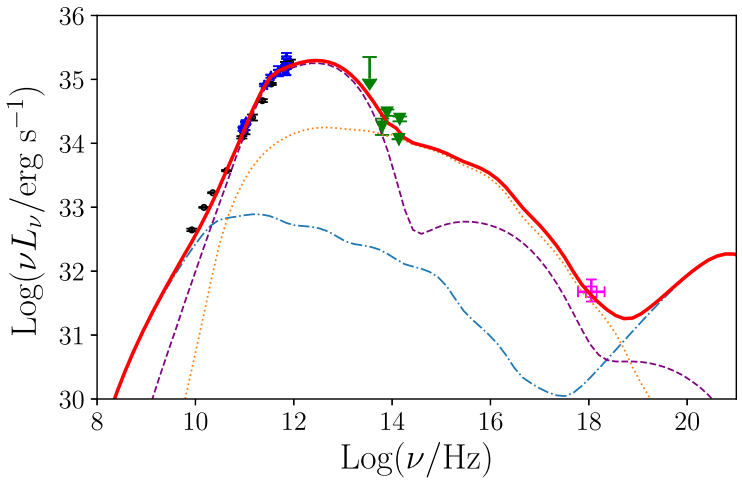

Figure 1. SED of Sgr $\mathrm{A}^{*}$ in the quiescent state. The dotted line is the emission of nonthermal electrons from the inner parts of the flow $\left(r<15 r_{\mathrm{S}}\right)$. The dashed line is the thermal synchrotron and inverse Compton emission. The dotteddashed line is the emission from the outer parts of the flow $\left(r>15 r_{\mathrm{S}}\right)$, including thermal bremsstrahlung and nonthermal synchrotron. The solid line is the total emission.

where $N_{\mathrm{q}}$ is the number density of electrons in the quiescent state, $\gamma$ is the electron Lorentz factor, $p$ is the spectral index at injection, $\gamma_{\mathrm{c}}$ is the "cooling break" Lorentz factor at which the the accretion time is equal to the cooling time, $t_{\mathrm{acc}}=t_{\mathrm{cool}}\left(\gamma_{\mathrm{c}}\right)$ (cf. Section 4), and $\gamma_{\min }$ and $\gamma_{\max }$ denote the minimum and maximum Lorentz factors, respectively. We assume that thermal electrons radiate locally through synchrotron, bremsstrahlung, and inverse Compton processes. For nonthermal electrons, we only consider synchrotron emission and adopt $p=3.6$.

Figure 1 shows the quiescent state SED for the parameters given above. The observations are from Liu et al. (2016) (radio, dark circles), Shcherbakov et al. (2012) (radio, blue dots), Schödel et al. (2011) (IR, green triangles), and Roberts et al. (2017) (X-rays, magenta square). The submillimeter bump is due to thermal synchrotron, and the radio and IR excess are nonthermal synchrotron radiation.

\subsection{Flare}

Our model for flaring emission assumes that an unspecified process converts a fraction of electrons from the Maxwellian distribution to a nonthermal one during a short burst-a "nonthermal bomb." In Section 4 we discuss about the possible physical mechanisms that might have produced such an event.

We consider that the burst occurs over an extended region ranging from radius $r_{\text {in }}$ to $r_{\text {out }}$. The injection function of nonthermal particles during a burst is

$$
\dot{N}_{\mathrm{b}}(\gamma, r ; t)=\dot{N}_{\mathrm{b}}(\gamma, r) \delta(t),
$$

where $\dot{N}_{\mathrm{b}}(\gamma, r)=K_{\mathrm{b}}(r) \gamma^{-p_{\mathrm{b}}}$, and $K_{\mathrm{b}}(r)$ is determined imposing that at each shell a fraction $\eta_{\mathrm{b}}>\eta_{\mathrm{q}}$ of the thermal energy goes to nonthermal particles. We follow the population while it is accreted onto the event horizon and compute the time evolution of the synchrotron emission. The transport equation that governs the evolution of this population is

$$
\begin{aligned}
& \frac{\partial N_{\mathrm{b}}(\gamma, r ; t)}{\partial t}+\frac{1}{r^{2}} \frac{\partial}{\partial r}\left[r^{2} v(r) N_{\mathrm{b}}(\gamma, r ; t)\right] \\
& +\frac{\partial}{\partial \gamma}\left[\left(\frac{d \gamma}{d t}\right)_{\mathrm{syn}} N_{\mathrm{b}}(\gamma, r ; t)\right]=\dot{N}_{\mathrm{b}}(\gamma, r) \delta(t),
\end{aligned}
$$

where $d \gamma / d t(\gamma, r)$ is the rate of energy losses by synchrotron emission and $v(r)$ is the radial velocity of the flow. We solve Equation (3) by the method of characteristics. There are five free parameters in the flare model: $\eta_{\mathrm{b}}$, the spectral index $p_{\mathrm{b}}, r_{\mathrm{in}}$, $r_{\text {out }}$, and $t_{0}$, which is the time at which the burst occurs.

\section{Results}

Figure 2 contains the main result of this Letter: we successfully explain the unprecedented bright state of Sgr A* observed in the NIR in 2019 May as an injection burst of nonthermal particles in the RIAF, which subsequently undergo radiative cooling as they get advected onto the $\mathrm{BH}$. The figure shows three models with different initial sizes of the burst region that reproduce well the decay in the NIR emission. The models reproduce the abrupt decrease in the flux in the last 10 minutes of observations. This is interpreted as the accretion of the last nonthermal particles accelerated in the burst-those near $r_{\text {out }}$ at $t=0$.

Our nonthermal bomb model predicts that the duration of the flare-determined by the accretion time-is the same across all wavelengths. The model also predicts that the slope of the LC following the initial burst depends on the wavelength. Both of these features are seen in Figure 3, which shows LCs in three different wavelengths: NIR, $1.3 \mathrm{~mm}$ (the Event Horizon Telescope wavelength), and $2-8 \mathrm{keV}$ (the Chandra and XMM-Newton energy band). The NIR LC is relatively insensitive to the slope of the electron energy distribution function, such that $L_{\mathrm{NIR}} \propto t^{-0.7}$. On the other hand, we find that the radio emission at millimeter wavelengths depends modestly on the power-law index $p_{\mathrm{b}}$. This dependence can be approximated as $L_{\mathrm{mm}} \propto t^{0.4-0.25 p_{\mathrm{b}}}$. The X-ray LC follows $L_{\mathrm{X}} \propto t^{0.4}$ and depends weakly on $p_{\mathrm{b}}$. Therefore, a campaign of multiwavelength monitoring of Sgr A*s $\mathrm{LC}$ following a superflare in radio, NIR, and X-rays should allow a concrete test of our model.

Figure 3 also demonstrates that there is more than one combination of parameters capable of reproducing the NIR observations. For instance, the effect of the parameters $p_{\mathrm{b}}$ and $\eta_{\mathrm{b}}$ on the LC is degenerate: a change in any of these parameters affects only the total luminosity at the $K_{\mathrm{s}}$ band but does not modify the slope of the LC. This degeneracy can be broken by monitoring $\mathrm{Sgr} \mathrm{A}^{*}$ following the outset of the nonthermal bomb at other wavelengths. A change in $\eta_{b}$ only, leaving $p_{\mathrm{b}}$ fixed, modifies the total amount of energy in the bomb, and thus the luminosity at all times and wavelengths. This is shown in Figure 3.

\section{Discussion}

\subsection{Acceleration Mechanism}

What is the mechanism responsible for for triggering the nonthermal bomb in $\mathrm{Sgr} \mathrm{A}^{*}$ ? $\mathrm{BH}$ accretion flows are highly turbulent, highly magnetized, relativistic environments (e.g., Porth et al. 2019). Thus, plausible culprits are magnetic reconnection events and/or turbulence acceleration. In fact, magnetic reconnection has been invoked to explain the recurring IR and X-ray flares observed in Sgr $\mathrm{A}^{*}$ (e.g., Ball et al. 2018). Shocks are unlikely because while being efficient at dissipating energy, they do not accelerate particles far beyond thermal energies (e.g., Sironi et al. 2015).

Numerical solutions of the Vlasov equation for astrophysical plasmas-i.e., particle-in-cell (PIC) simulations-are showing 


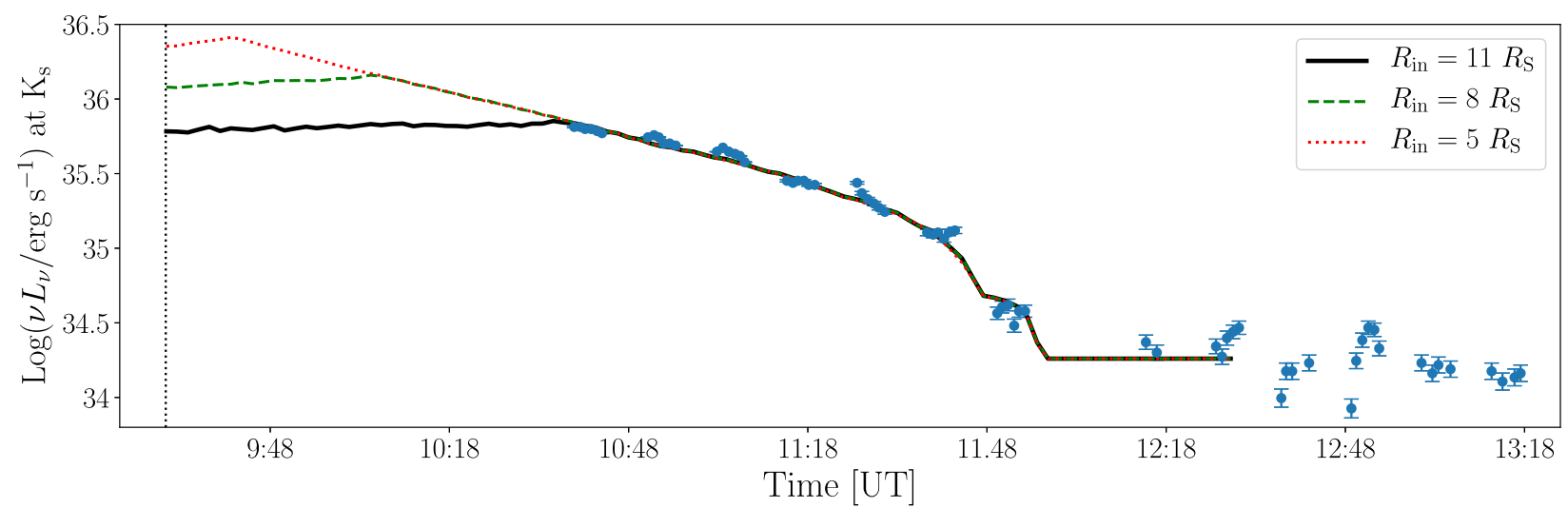

Figure 2. NIR LC of the superflare of Sgr A*. Points correspond to the Keck Telescope observations of Do et al. (2019) and lines indicate different nonthermal bomb models. The model parameters are $\eta_{b}=0.25, p_{\mathrm{b}}=2.05$, and $r_{\text {out }}=16 r_{\mathrm{S}}$, for three different values of $r_{\text {in }}$.
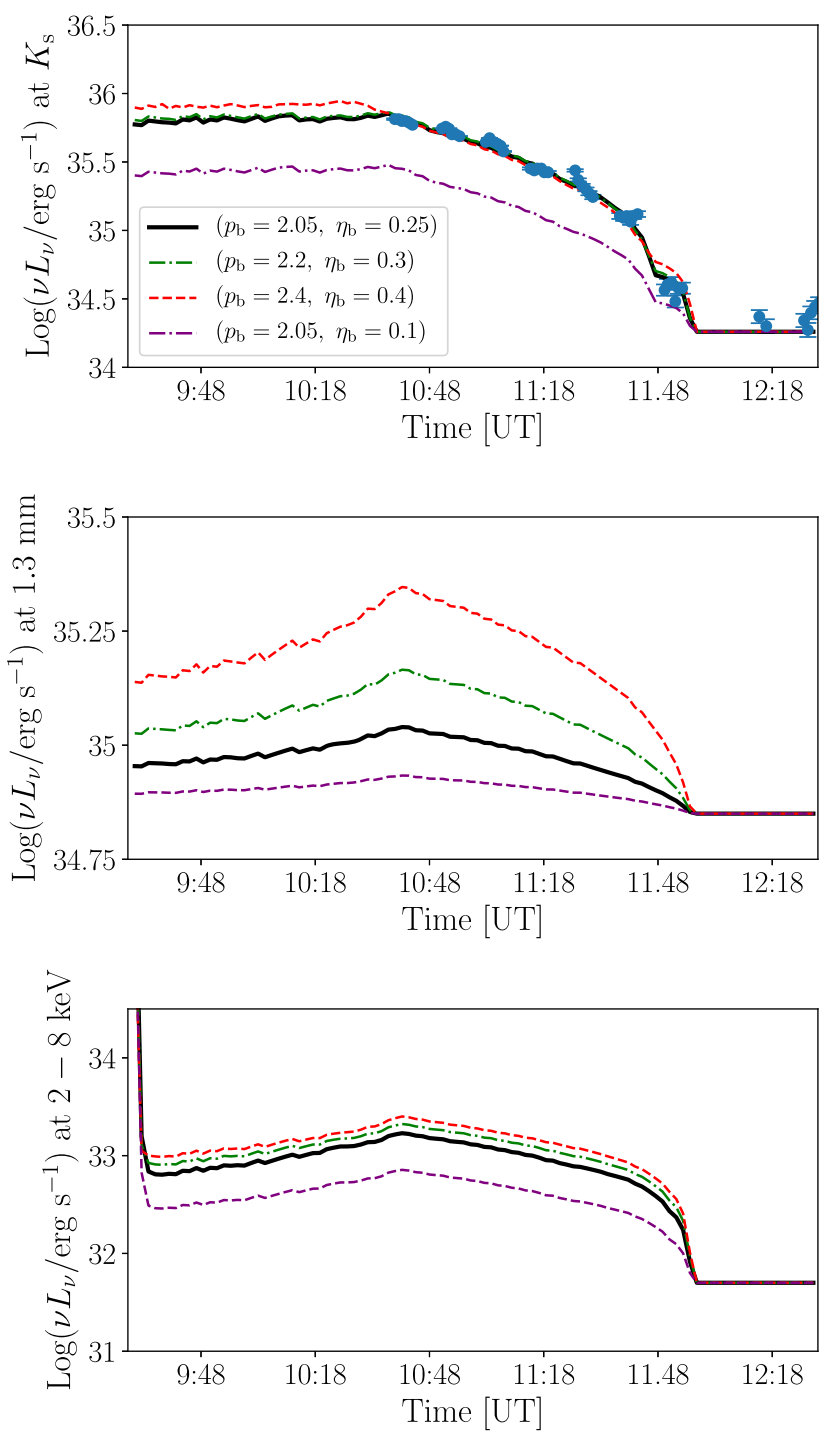

Figure 3. Predicted flare emission at three wavelengths: NIR (upper panel), 1.3 $\mathrm{mm}$ (middle panel), and X-rays (2-8 keV; lower panel). Three different values of the spectral index of the nonthermal distribution are displayed plus a model with the same spectral index as our fiducial model but with a lower value of $\eta_{\mathrm{b}}$.

that: (i) magnetic reconnection events with high magnetizations ${ }^{3}$ of $\sigma \gtrsim 10$ lead to particles following power-law energy distributions with an index $p$ ranging from 1 to 2 (e.g.,
Guo et al. 2014; Sironi \& Spitkovsky 2014), (ii) the presence of relativistic ${ }^{4}$ turbulence acceleration leads to a power-law index closer to 2 (Comisso \& Sironi 2019), and (iii) reconnection can deposit a large fraction (up to about 50\%) of the dissipated energy in nonthermal electrons.

We have found that models with $p$ between 2 and 2.5 and $\eta_{b} \approx 0.25$ can account for the NIR flare evolution. Energy distributions with these parameters are consistent with having been produced within 10 gravitational radii of the event horizon by either a magnetic reconnection event, or a reconnection event followed by relativistic turbulence acceleration.

For instance, according to the PIC simulations of Petropoulou et al. (2016) a lone reconnection event with $\sigma \approx 10$ should produce nonthermal electrons with the required values of $p$ and $\eta_{\mathrm{b}}$. Global GRMHD simulations such as those carried out by Ball et al. (2018) demonstrate that $\sigma$ is correlated with the plasma- $\beta, \beta \equiv P_{\text {gas }} / P_{\text {magnetic. The values of } \sigma \gtrsim 10 \text { required to }}$ explain the superflare are only attained in configurations with high amounts of magnetic flux near the event horizon-i.e., the magnetically arrested disk (MAD) state-in regions of the accretion flow at which $\beta \sim 0.1$ (Ball et al. 2018). In our fiducial LC model, the total amount of magnetic energy involved in the burst is $\sim 3 \times 10^{40} \mathrm{erg} \mathrm{s}^{-1}$. The MAD models of Ball et al. reach at most $\sim 10^{39} \mathrm{erg} \mathrm{s}^{-1}$ for $\sigma \approx 10$; therefore, a nonthermal bomb needs unusually large values of $B$-three times larger than the peak values of $B$ reached in MAD models. This would explain why superflares such as the one observed in 2019 May should be quite rare.

\subsection{Timescales}

The relevant timescales for our problem are the electron cooling time and the accretion time. Interestingly, during the nonthermal bomb these timescales should be comparable. The synchrotron cooling time for an electron of Lorentz factor $\gamma$ is

$$
t_{\mathrm{syn}} \approx 7.74 \times 10^{6}\left(\frac{B}{10 \mathrm{G}}\right)^{-2} \gamma^{-1} \mathrm{~s} .
$$

\footnotetext{
3 The magnetization parameter is defined as $\sigma \equiv B^{2} / 4 \pi \rho c^{2}$, where $B$ is the magnetic field intensity and $\rho$ is the mass density-all quantities measured in the rest frame of the fluid.

4 Hereafter, by relativistic we mean that the mean magnetic energy per particle is larger than the rest-mass energy.
} 
The cooling time corresponds to

$$
t_{\mathrm{syn}} \approx\left(\frac{B}{10 \mathrm{G}}\right)^{-3 / 2} \mathrm{hr} \text {. }
$$

For magnetic fields of the order of $10 \mathrm{G}$, as appropriate for Sgr $\mathrm{A}^{*}$ at $\approx 10 r_{\mathrm{S}}$, the cooling time is of the order of one hour. The accretion timescale is defined as $t_{\text {acc }}=R /|v|$. Using the selfsimilar RIAF solution (Narayan \& Yi 1994) we obtain a firstorder estimate of this timescale as

$$
t_{\mathrm{acc}} \approx 3 \alpha r^{3 / 2} \mathrm{hr} \text {. }
$$

For $\alpha=0.1$ and $r \approx 10, t_{\mathrm{acc}} \sim 10 \mathrm{hr}$. In the models displayed in Figure 2, the duration of the flare is determined mainly by the accretion time, but the slope also depends on the electron cooling. However, we find that a model only taking into account cooling with electrons remaining at a fixed distance from the hole-i.e., undergoing convective motion-also fits well the data. This shows that cooling can have an effect as important as accretion in our model.

\section{Summary}

Sgr A* has experienced a strong, unprecedented flare in 2019 May when its near-IR luminosity reached much brighter levels than ever measured. We have explained this superflare with a nonthermal bomb model, where an unspecified process accelerates over a very short time a small fraction of the electrons into a nonthermal distribution; these electrons subsequently cool and are advected onto the BH. Besides explaining the NIR LC, our model predicts that the radio and $\mathrm{X}$-ray fluxes should decay over time in a similar fashion. In particular, the radio LC at millimeter wavelengths is sensitive to the particle energy distribution and dissipation efficiency.

The nonthermal bomb detonated in a region spanning a length $5 R_{S}$ in the innermost parts of the accretion flow, and is likely due to a magnetic reconnection event involving unusually strong magnetic fields and high magnetization, i.e., $\sigma$ $\gtrsim 10$, or such a reconnection event followed by turbulence acceleration.

A multiwavelength monitoring of such superflares in radio, NIR, and X-rays should allow a concrete test of the nonthermal bomb model and better constrain the mechanism that triggered the bomb. Future theoretical research should investigate the observational signatures of relativistic reconnection and relativistic turbulence acceleration using realistic magnetic field configurations appropriate for the SMBH in our Galactic Center, combining the tools of multidimensional GRMHD and PIC simulations.

We thank Reinaldo Santos de Limia for useful discussions, Fan Guo for providing us useful references, and the anonymous referee for very constructive comments that led to improvements in this Letter. E.G. thanks Gustavo Romero and Florencia Vieyro for useful discussions about relativistic processes in the vicinity of black holes. This work was supported by the Argentine National Scientific and Technical Research Council (CONICET, grant PIP 2014-00338), the National Agency for Scientific and Technological Promotion (PICT 2017-0898), Fundação de Amparo à Pesquisa do Estado de São Paulo (FAPESP, grant 2017/01461-2), and Conselho Nacional de Desenvolvimento Científico e Tecnológico (CNPq, grant 142320/2016-1).

\section{ORCID iDs}

Eduardo M. Gutiérrez (10 https://orcid.org/0000-00017941-801X

Rodrigo Nemmen (iD https://orcid.org/0000-0003-3956-0331

Fabio Cafardo (iD https://orcid.org/0000-0002-7910-2282

\section{References}

Abuter, R., Amorim, A., Bauboeck, M., et al. 2019, in Proc. XIII Scientific Meeting of the Spanish Astronomical Society, Highlights on Spanish Astrophysics X, ed. B. Montesinos et al. (Berlin: Springer), 609

Ball, D., Özel, F., Psaltis, D., Chan, C.-K., \& Sironi, L. 2018, ApJ, 853, 184 Blandford, R. D., \& Begelman, M. C. 1999, MNRAS Letters, 303, L1

Boyce, H., Haggard, D., Witzel, G., et al. 2018, arXiv:1812.05764

Comisso, L., \& Sironi, L. 2019, ApJ, 886, 122

Dibi, S., Markoff, S., Belmont, R., et al. 2014, MNRAS, 441, 1005

Do, T., Witzel, G., Gautam, A. K., et al. 2019, ApJL, 882, L27

Eckart, A., Baganoff, F. K., Schödel, R., et al. 2006, A\&A, 450, 535

Falcke, H., \& Markoff, S. B. 2013, CQGra, 30, 244003

Fazio, G. G., Hora, J. L., Witzel, G., et al. 2018, ApJ, 864, 58

Genzel, R., Schödel, R., Ott, T., et al. 2003, Natur, 425, 934

Guo, F., Li, H., Daughton, W., \& Liu, Y.-H. 2014, PhRvL, 113, 155005

Liu, H. B., Wright, M. C. H., Zhao, J.-H., et al. 2016, A\&A, 593, A107

Narayan, R., \& Yi, I. 1994, ApJL, 428, L13

Neilsen, J., Nowak, M. A., Gammie, C., et al. 2013, ApJ, 774, 42

Nowak, M. A., Neilsen, J., Markoff, S. B., et al. 2012, ApJ, 759, 95

Petropoulou, M., Giannios, D., \& Sironi, L. 2016, MNRAS, 462, 3325

Ponti, G., De Marco, B., Morris, M. R., et al. 2015, MNRAS, 454, 1525

Ponti, G., George, E., Scaringi, S., et al. 2017, MNRAS, 468, 2447

Porth, O., Chatterjee, K., Narayan, R., et al. 2019, ApJS, 243, 26

Ressler, S. M., Quataert, E., \& Stone, J. M. 2018, MNRAS, 478, 3544

Roberts, S. R., Jiang, Y.-F., Wang, Q. D., \& Ostriker, J. P. 2017, MNRAS, 466, 1477

Schödel, R., Morris, M. R., Muzic, K., et al. 2011, A\&A, 532, A83

Shcherbakov, R. V., Penna, R. F., \& McKinney, J. C. 2012, ApJ, 755, 133

Sironi, L., Keshet, U., \& Lemoine, M. 2015, SSRv, 191, 519

Sironi, L., \& Spitkovsky, A. 2014, ApJL, 783, L21

Stone, J. M., Marrone, D. P., Dowell, C. D., et al. 2016, ApJ, 825, 32

Yuan, F., \& Narayan, R. 2014, ARA\&A, 52, 529

Yuan, F., Quataert, E., \& Narayan, R. 2003, ApJ, 598, 301

Yusef-Zadeh, F., Arendt, R., Bushouse, H., et al. 2012, ApJL, 758, L11

Yusef-Zadeh, F., Roberts, D., Wardle, M., Heinke, C. O., \& Bower, G. C. 2006, ApJ, 650, 189

Yusef-Zadeh, F., Wardle, M., Heinke, C., et al. 2008, ApJ, 682, 361 
Appendix $\mathrm{C}$

\section{New sources found in the analysis between $100 \mathrm{MeV}$ and $500 \mathrm{GeV}$}

Here we present the new sources encountered in the RoI with Fermipy's find_sources function in the energy range between $100 \mathrm{MeV}$ and $500 \mathrm{GeV}$ (our Universal Model). The best parameters for the power-law used to model their spectra are also shown together with their TS and position. 


\begin{tabular}{ccccccc}
\hline \hline Source name & Index & Prefactor & Scale & TS & RA & Dec \\
\hline PS J1719.1-2945 & -5.0 & $2.8 \times 10^{-14}$ & 1000 & 236 & 259.80 & -29.75 \\
PS J1720.6-2655 & -2.1 & $4.5 \times 10^{-13}$ & 1000 & 40 & 260.16 & -26.93 \\
PS J1723.8-3347 & -2.0 & $5.8 \times 10^{-13}$ & 1000 & 31 & 260.95 & -33.79 \\
PS J1729.5-3623 & -1.9 & $4.7 \times 10^{-13}$ & 1000 & 40 & 262.39 & -36.40 \\
PS J1730.5-2801 & -2.0 & $3.8 \times 10^{-13}$ & 1000 & 31 & 262.64 & -28.03 \\
PS J1731.6-2903 & -1.8 & $2.9 \times 10^{-13}$ & 1000 & 34 & 262.92 & -29.05 \\
PS J1733.8-2114 & -2.0 & $2.8 \times 10^{-13}$ & 1000 & 30 & 263.47 & -21.24 \\
PS J1734.4-3555 & -2.2 & $5.8 \times 10^{-13}$ & 1000 & 34 & 263.62 & -35.93 \\
PS J1734.6-2328 & -2.2 & $4.9 \times 10^{-13}$ & 1000 & 37 & 263.66 & -23.48 \\
PS J1735.5-2944 & -1.8 & $3.3 \times 10^{-13}$ & 1000 & 31 & 263.88 & -29.74 \\
PS J1735.6-2900 & -5.0 & $1.6 \times 10^{-14}$ & 1000 & 36 & 263.91 & -29.01 \\
PS J1742.7-3150 & -2.0 & $8.0 \times 10^{-13}$ & 1000 & 50 & 265.68 & -31.85 \\
PS J1744.1-3019 & -2.2 & $2.7 \times 10^{-12}$ & 1000 & 124 & 266.03 & -30.32 \\
PS J1747.2-2114 & -2.3 & $5.8 \times 10^{-13}$ & 1000 & 40 & 266.81 & -21.25 \\
PS J1750.4-3355 & -2.2 & $5.6 \times 10^{-13}$ & 1000 & 40 & 267.61 & -33.93 \\
PS J1752.0-3447 & -2.2 & $4.1 \times 10^{-13}$ & 1000 & 34 & 268.02 & -34.79 \\
PS J1752.6-2105 & -5.0 & $4.0 \times 10^{-14}$ & 1000 & 303 & 268.15 & -21.09 \\
PS J1754.4-2612 & -2.1 & $7.6 \times 10^{-13}$ & 1000 & 32 & 268.60 & -26.21 \\
PS J1754.4-2649 & -2.0 & $5.8 \times 10^{-13}$ & 1000 & 30 & 268.60 & -26.82 \\
PS J1754.7-3730 & -2.2 & $3.7 \times 10^{-13}$ & 1000 & 38 & 268.70 & -37.51 \\
PS J1755.3-2553 & -2.0 & $9.3 \times 10^{-13}$ & 1000 & 49 & 268.83 & -25.90 \\
PS J1756.0-3248 & -2.1 & $4.1 \times 10^{-13}$ & 1000 & 31 & 269.02 & -32.81 \\
PS J1756.3-2515 & -2.3 & $2.1 \times 10^{-12}$ & 1000 & 99 & 269.08 & -25.25 \\
PS J1756.8-2413 & -1.9 & $5.4 \times 10^{-13}$ & 1000 & 36 & 269.21 & -24.22 \\
PS J1758.0-2421 & -2.2 & $1.1 \times 10^{-12}$ & 1000 & 38 & 269.52 & -24.36 \\
PS J1808.2-3003 & -2.2 & $4.5 \times 10^{-13}$ & 1000 & 45 & 272.06 & -30.05 \\
PS J1817.4-2516 & -2.4 & $5.7 \times 10^{-13}$ & 1000 & 50 & 274.35 & -25.27 \\
PS J1818.6-2812 & -2.6 & $5.0 \times 10^{-13}$ & 1000 & 57 & 274.65 & -28.22 \\
PS J1823.9-2341 & -2.4 & $5.1 \times 10^{-13}$ & 1000 & 47 & 275.99 & -23.69 \\
\hline
\end{tabular}

Table C.1 - New sources found in the $100 \mathrm{MeV}$ to $500 \mathrm{GeV}$ energy range. 


\section{Appendix D}

\section{Examples of discontinuous models}

Here we show, for the sake of clarity only, a few examples of discontinuous models that we obtained in analyses in which the "minimal fitting" procedure was not used. In Figure D.1 we plot 4 examples of the energy flux models $\left(\mathrm{E}^{2} \mathrm{dN} / \mathrm{dE}\right)$ we obtained for single sources. Each energy range was treated as an isolated analysis. It is possible to see that the models are not continuous in every energy band (we are not showing the statistical uncertainties). These models were not used in our work. We developed the "minimal fitting" methodology to avoid results like these. 

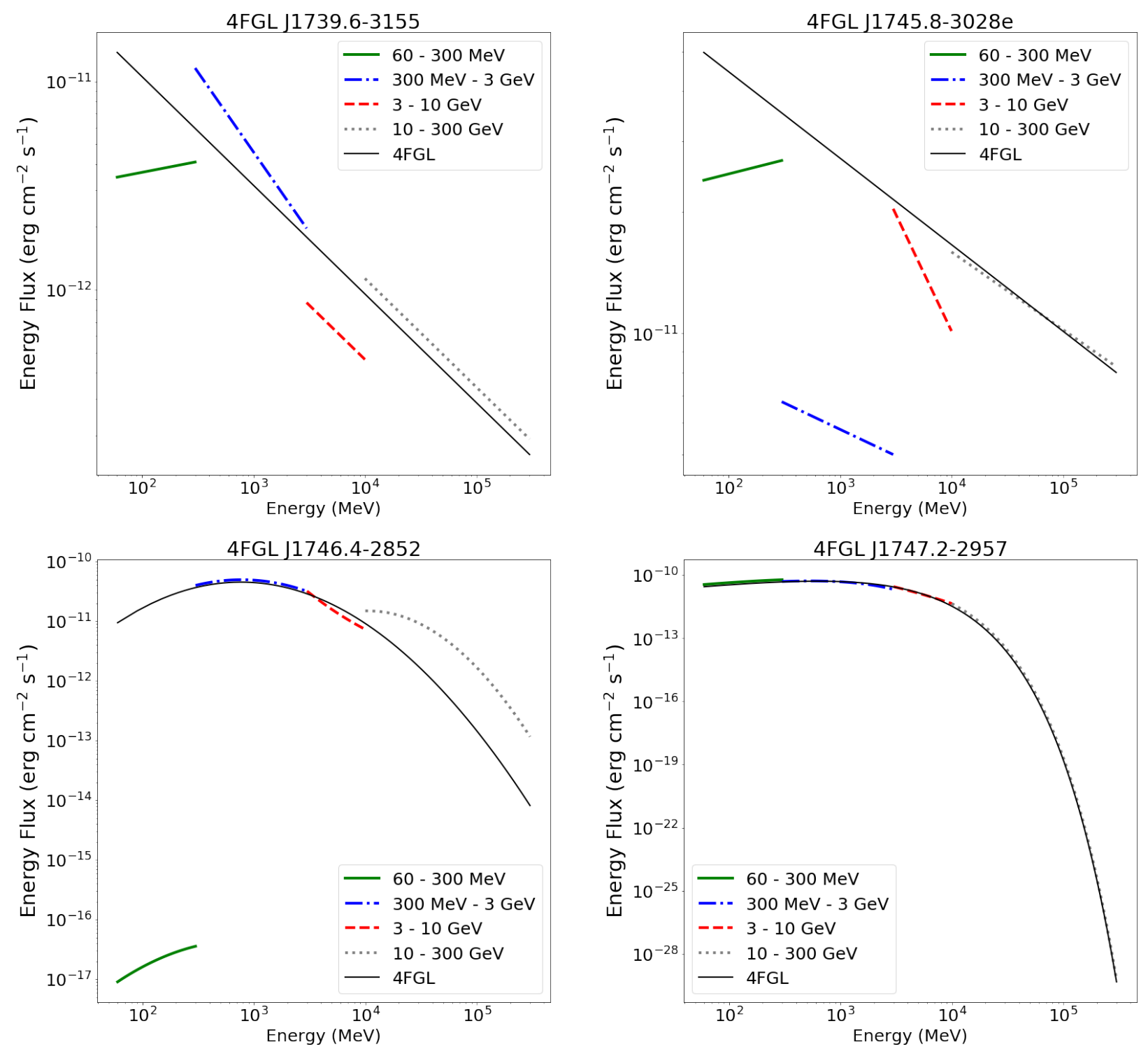

Figure D.1: Examples of energy flux models $\left(\mathrm{E}^{2} \mathrm{dN} / \mathrm{dE}\right)$ we obtained for single sources when we separated the analyses into four independent energy band. These models were not used in our work. 
Appendix E

\section{Comparing the results obtained through the minimal fitting method to a more standard approach}

Here we present the results of previous analyses performed for the three energy bands (i.e., $300 \mathrm{MeV}-3 \mathrm{GeV}, 3-10 \mathrm{GeV}$ and $10-500 \mathrm{GeV}$ ), based on models created form scratch to each one. In other words: models that were not created by splitting the Universal Model into tighter bands with the minimal fitting.

The photon selection for this analyses were very similar with the one used for the Universal Model, but not exactly equal: mainly, we used only $~ 10.5$ years of data and, for the two highest energy bands only, we used all event types available: PSF0 + PSF1 + PSF2 + PSF3, which means all photons. Regardless these (and other minor) differences in the data selection and in the analyses, the results we obtained were coincident within 1 $\sigma$ with the what we achieve with the reported in the Section 3.

We start with the diagnostic plots. In Figure E.1 we show the residual maps for the models created from scratch for these 3 energy bands. In Figure E.2 we show the TS maps with the central source included in the models. And, in Figure E.3 we show the TS maps without the source in the models. Theses Figures were created following the same procedure described in Section 2.3.3 for the creation of Figures 2.4, 2.6 and 2.8, respectively.

The gamma-ray fluxes measured in these previous analyses are presented in Table E.1. All of them are compatible within $1 \sigma$ with the results presented in Table 3.1, that we obtained through the splitting of the Universal Model.

We can conclude, then, that the results we obtained with the offspring of the Universal Model are consistent even though their diagnostic plots are not flawless. 

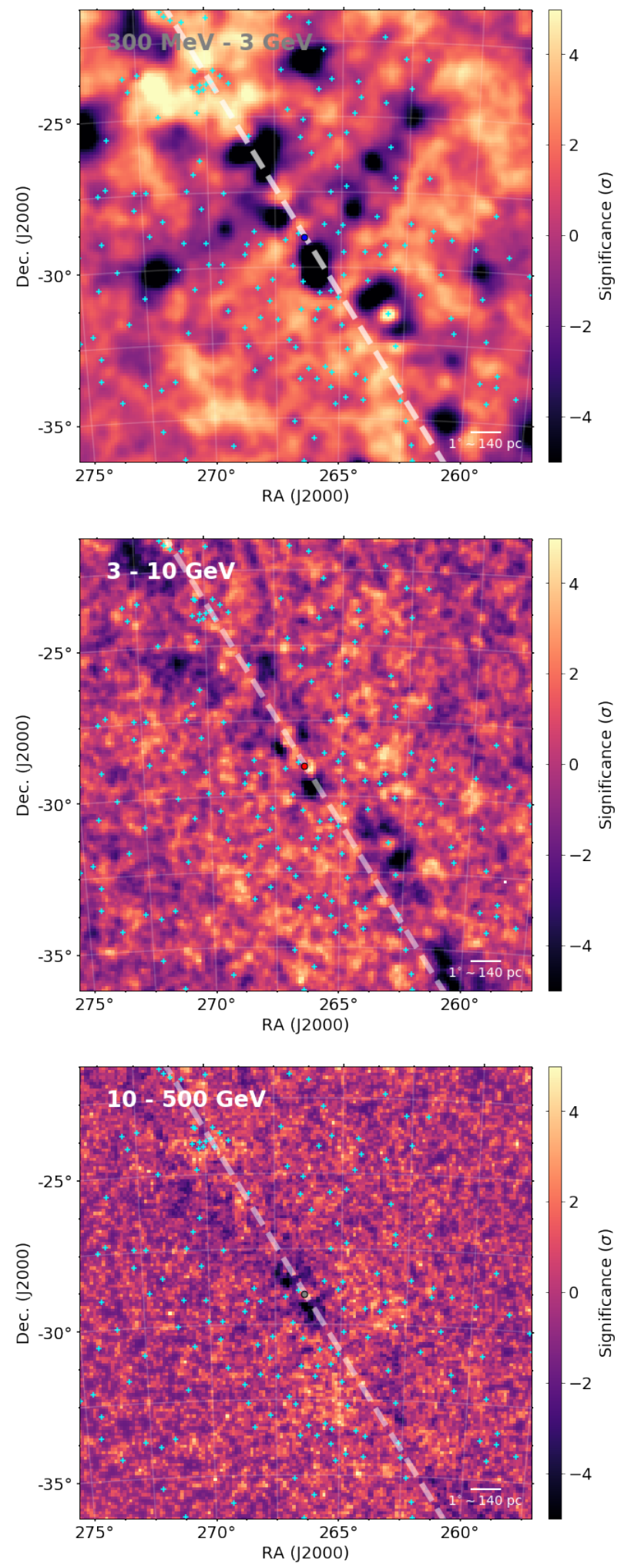

Figure E.1: Residuals maps for previous analyses in the 3 highest energy ranges. The colors show the significance of the residual. The point at the center of each panel corresponds to the source position obtained in each energy range. 4FGL point sources are displayed as cyan crosses. The white dashed lines indicate the direction of the Galactic plane. 

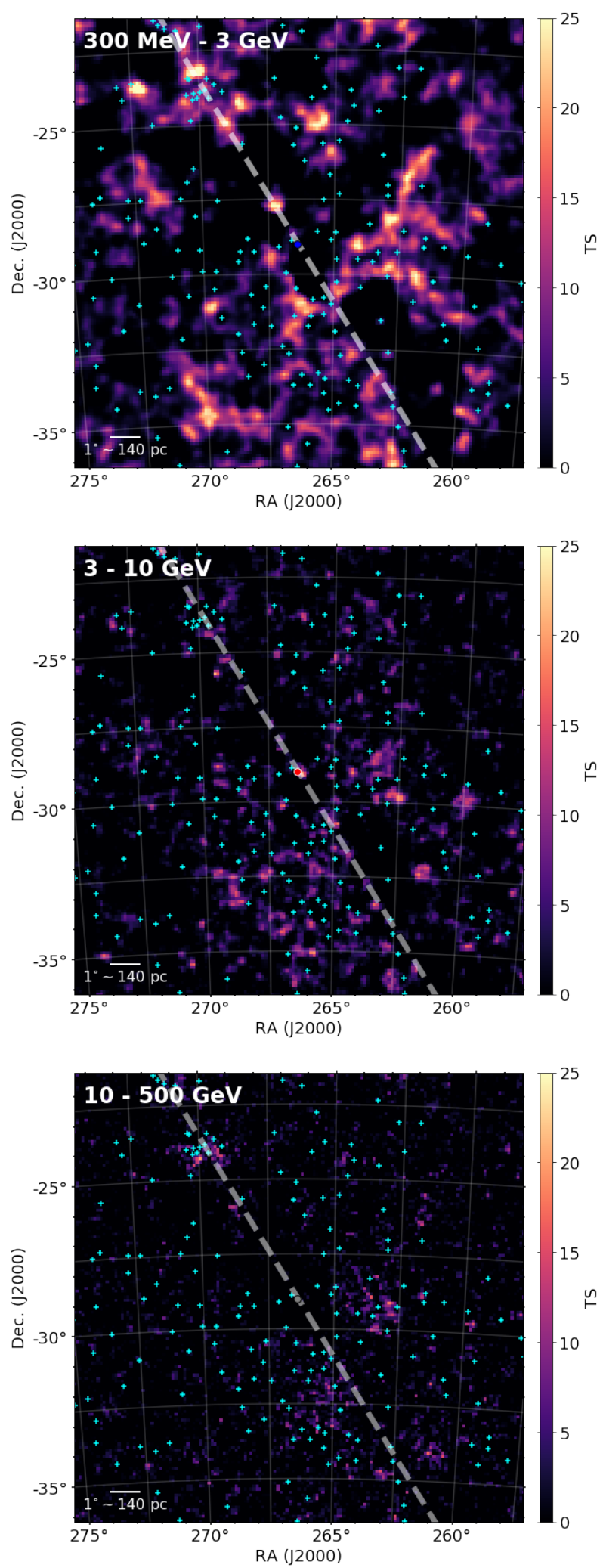

Figure E.2: TS maps of the RoI for previous analyses in the 3 highest energy ranges. The circles at the center of the panels correspond to the central point source position obtained in each energy range. 4FGL point sources are displayed as cyan crosses. The white dashed lines indicate the direction of the Galactic plane. 

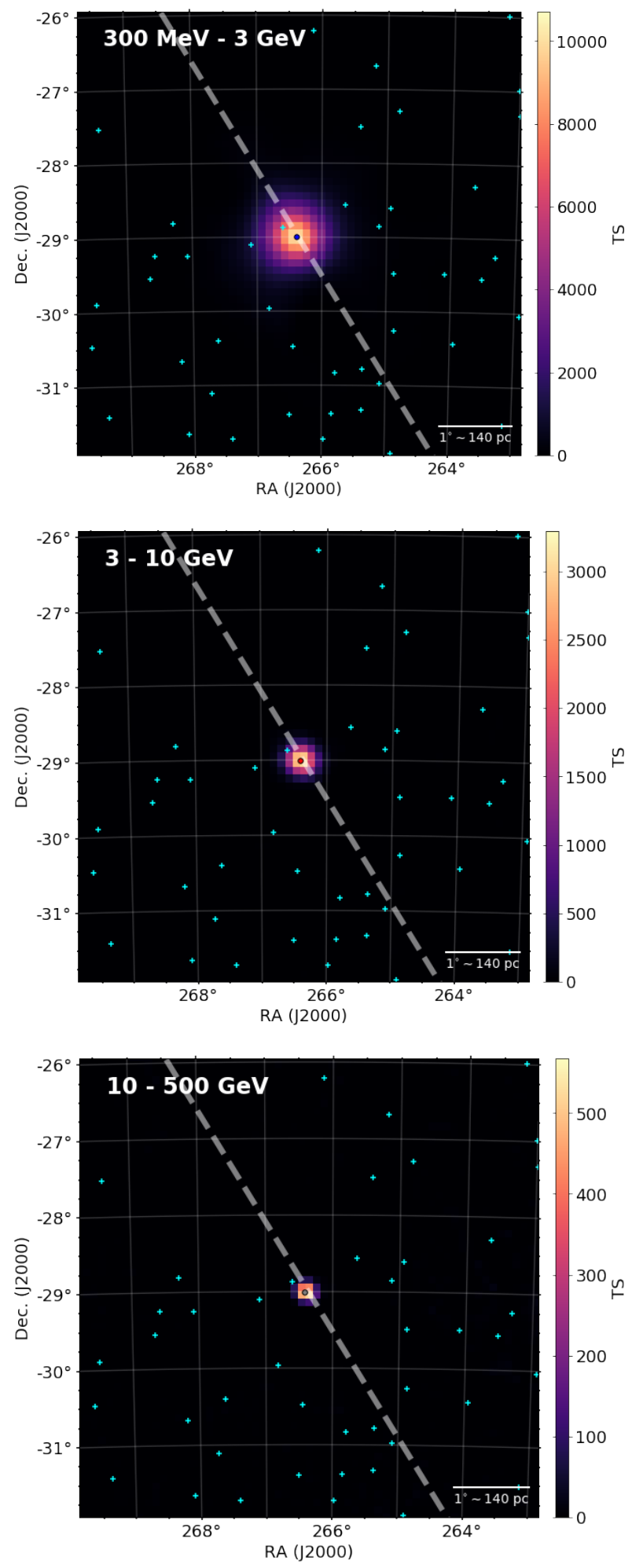

Figure E.3: TS maps of the inner $8^{\circ} \times 8^{\circ}$ of the RoI evidencing the contribution of the central point source. They were constructed after excluding 4FGL J1745.6-2859 from the models, as explained in Section 2.3.3 The point at the center of each panel corresponds to the source position obtained in each energy range. The other 4FGL point sources are shown as cyan crosses. The white dashed lines indicate the direction of the Galactic plane. 
Table E.1 - Results from likelihood modeling of the central point source for previous analyses in the 3 highest energy bands. The photon and energy flux uncertainties are statistical only. All the results are compatible within $1 \sigma$ with the results presented in Table 3.1 .

\begin{tabular}{ccccccc}
\hline \hline $\begin{array}{c}\text { Energy } \\
\text { range }(\mathrm{GeV})\end{array}$ & TS $^{1}$ & $\begin{array}{c}\text { Photon flux } \\
\left(\mathrm{cm}^{-2} \mathrm{~s}^{-1}\right)\end{array}$ & $\begin{array}{c}\text { Energy flux } \\
\left(\mathrm{erg} \mathrm{cm}^{-2} \mathrm{~s}^{-1}\right)\end{array}$ & $\begin{array}{c}\text { Centroid }^{2} \\
\left({ }^{\circ}\right)\end{array}$ & \multicolumn{2}{c}{ Positional Uncertainty $^{3}$} \\
statistical $\left(^{\circ}\right)$ & total $\left.^{\circ}\right)$ \\
\hline $0.3-3$ & 10132 & $(1.67 \pm 0.03) \times 10^{-7}$ & $(2.04 \pm 0.03) \times 10^{-10}$ & $266.403,-28.995$ & 0.005 & 0.009 \\
$3-10$ & 3398 & $(8.86 \pm 0.24) \times 10^{-9}$ & $(6.66 \pm 0.18) \times 10^{-11}$ & $266.404,-29.002$ & 0.005 & 0.009 \\
$10-500$ & 720 & $(1.14 \pm 0.07) \times 10^{-9}$ & $(4.22 \pm 0.42) \times 10^{-11}$ & $266.411,-29.006$ & 0.006 & 0.010 \\
\hline
\end{tabular}

$\sqrt{T S} \approx$ detection significance of the source in each energy range

${ }^{2} \mathrm{RA}$ and Dec. corresponding to the emission centroid in degrees

${ }^{3} 68 \%$ positional uncertainty 
Appendix F

\section{New sources found in the analysis between 60 and 300 $\mathrm{MeV}$}

Five new sources were encountered in the RoI with Fermipy's find_sources function in the energy range between 60 and $300 \mathrm{MeV}$. They are listed in this section. The best parameters for the power-law used to model their spectra are also shown together with their TS and position.

Table F.1 - New sources found in the 60 to $300 \mathrm{MeV}$ energy range.

\begin{tabular}{ccccccc}
\hline \hline Source name & Index & Prefactor & Scale & TS & RA & Dec \\
\hline PS J1639.5-2448 & -1.7 & $4.4 \times 10^{-12}$ & 1000 & 37 & 249.90 & -24.81 \\
PS J1750.6-2723 & -2.0 & $4.8 \times 10^{-12}$ & 1000 & 27 & 267.65 & -27.40 \\
PS J1753.7-2127 & -0.9 & $2.9 \times 10^{-12}$ & 1000 & 57 & 268.44 & -21.47 \\
PS J1820.5-2113 & -2.0 & $4.3 \times 10^{-12}$ & 1000 & 61 & 275.14 & -21.22 \\
PS J1835.0-1804 & -5.0 & $8.8 \times 10^{-15}$ & 1000 & 183 & 278.76 & -18.07 \\
\hline
\end{tabular}


Appendix G

\section{Comparing different event type selection for the low energy model}

Here we present the results we obtained with preliminary analyses for the $60-300 \mathrm{MeV}$ energy range. We were interested in testing the impact of different event types selection. The event types we used in the preliminary tests were:

- PSF1 + PSF2 + PSF3: which is the selection we decided to use in the main analysis

- PSF2 + PSF3: which means the $50 \%$ photons with better PSF in the data

- front: that consider only the events that converted in the Fermi-LAT's "front", a part of the instrument that provides counts with better spatial resolution, as explained in section 1.5 .1

Since these were just tests, we tried to be as quick as possible. So we performed only a few rounds of fitting, among other differences in the modeling process presented in Section 2 (for instance, we did not use Fermipy's function find_sources and did not change the log-parabola spectral model to a power-law).

In Figure G.1 we show the position of the source in the three tests and, also, the position we are reporting in this work (Section 3.2). The results are very similar among every photon selection: the point sources are all coincident within their $68 \%$ positional uncertainty (statistical and systematic uncertainties).

The tests results are all clustered in the same region, about $\sim 0^{\circ} .05$ away from the result we obtained later with a complete fitting (green line). The fact that the result of the test with event type PSF1 + PSF2 + PSF3 is also clustered with the others tests indicates 
that the offset of our final result was not caused by the photon selection but, instead, is a result of the better modeling.

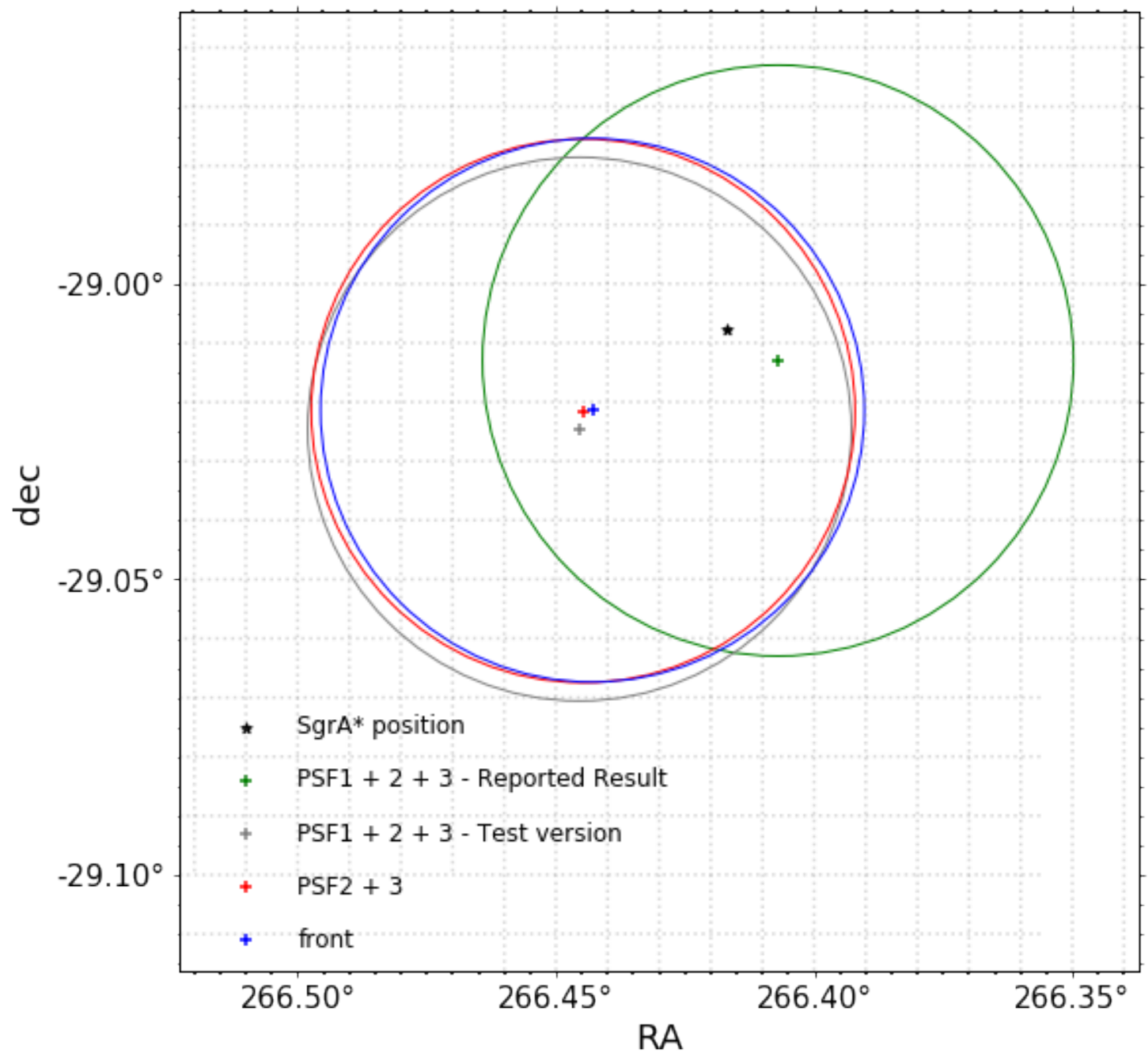

Figure G.1: The position of the central source in the 60-3000 MeV energy band for as reported in this work (green) and for the event type tests (gray, red and blue). The circles represent the $68 \%$ positional uncertainty. The radio position of Sgr A* is indicated by the black cross.

In Table G.1 we report the results we obtained for the central source's photon and energy fluxes in these tests. All the results for the PSF1 + PSF2 + PSF3 test are compatible within $1 \sigma$ with the final results presented in Table 3.1 for the $60-300 \mathrm{MeV}$. For the other tests, only the photon flux is compatible within $1 \sigma$, while the energy flux is compatible within $2 \sigma$ with the results presented in Table 3.1 for the same energy range. We are reporting only the statistical uncertainties, so they are likely underestimated. 
Table G.1 - Results from the event types testing for the 60-300 MeV energy interval. The photon and energy flux uncertainties are statistical only. All the results for the PSF1 + PSF2 + PSF3 test are compatible within $1 \sigma$ with the results presented in Table 3.1. For the other tests, only the photon flux is compatible within $1 \sigma$, while the energy flux is compatible within $2 \sigma$ with the results presented in Table 3.1 for the same energy range.

\begin{tabular}{cccc}
\hline \hline Event type & TS $^{1}$ & $\begin{array}{c}\text { Photon flux } \\
\left(\mathrm{cm}^{-2} \mathrm{~s}^{-1}\right)\end{array}$ & $\begin{array}{c}\text { Energy flux } \\
\left(\mathrm{erg} \mathrm{cm}^{-2} \mathrm{~s}^{-1}\right)\end{array}$ \\
\hline PSF1 + PSF2 + PSF3 & 2525 & $(5.25 \pm 0.13) \times 10^{-7}$ & $(1.09 \pm 0.02) \times 10^{-10}$ \\
PSF2 + PSF3 & 2250 & $(5.50 \pm 0.17) \times 10^{-7}$ & $(1.14 \pm 0.04) \times 10^{-10}$ \\
front & 2302 & $(5.62 \pm 0.21) \times 10^{-7}$ & $(1.17 \pm 0.04) \times 10^{-10}$ \\
\hline $1 \sqrt{T S} \approx$ detection significance of the source in each energy range
\end{tabular}


Appendix $\mathrm{H}$

Residuals distribution for the Universal Model offspring

In Figure H.1 we show the resulting residuals distribution after fitting the three offspring of the Universal Models. 

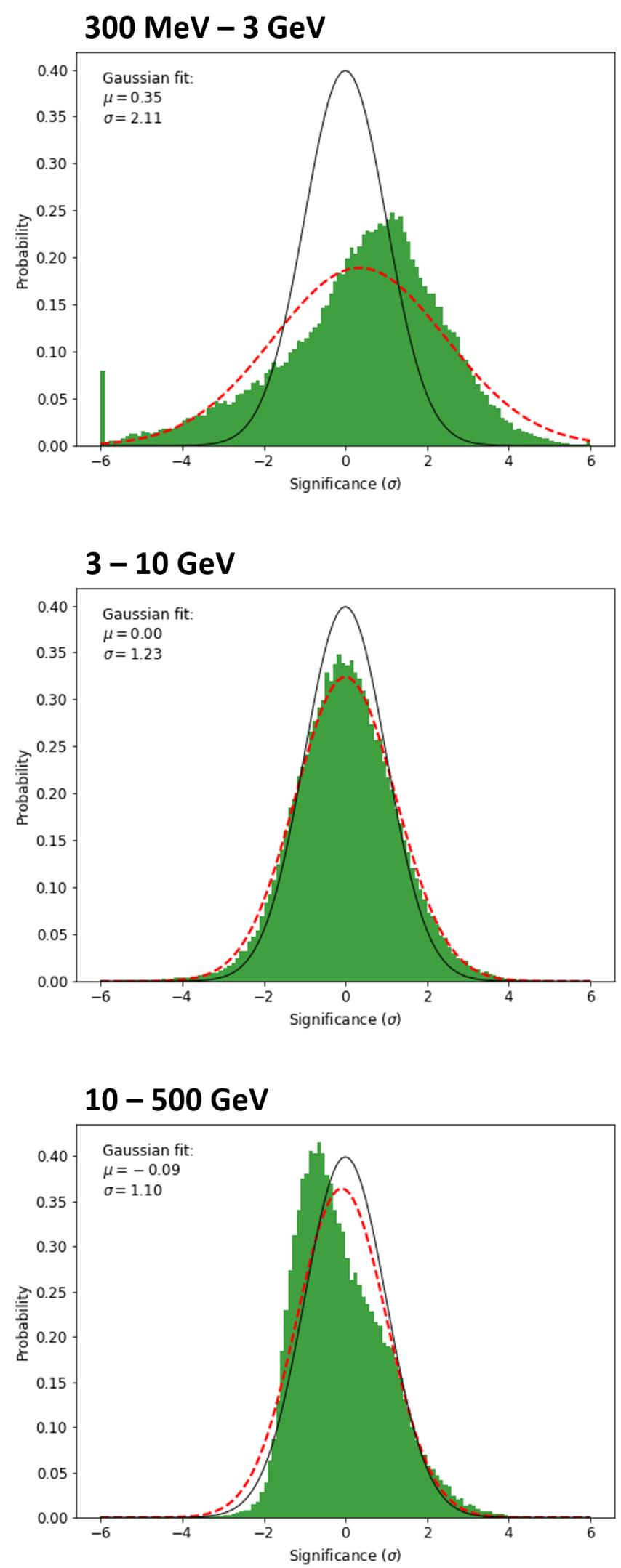

Figure H.1: Resulting residuals histograms obtained after fitting the three offspring of the Universal Model (i.e., top: $300 \mathrm{MeV}-3 \mathrm{GeV}$, middle: $3-10 \mathrm{GeV}$ and bottom: $10-500 \mathrm{GeV}$ ). The black lines are a standard normal distribution and the red dashed lines are the distributions for the best-fit Gaussian to the data. 


\section{Appendix I}

\section{Estimating the systematic uncertainties in the 15 days photon flux LC}

Here we perform the same analysis as in Section 2.5.2, but for the photon flux LC of 4FGL J1747.2-2957 in order to estimate the systematic uncertainty in the 15 days LC created with data in the $100 \mathrm{MeV}-500 \mathrm{GeV}$ energy range. We will only focus on showing the relevant plots, since the details of the analysis are in the main text.

As a result of this analysis, we obtained a systematic uncertainty of $14.8 \%$ for the photon flux 15 days LC. 


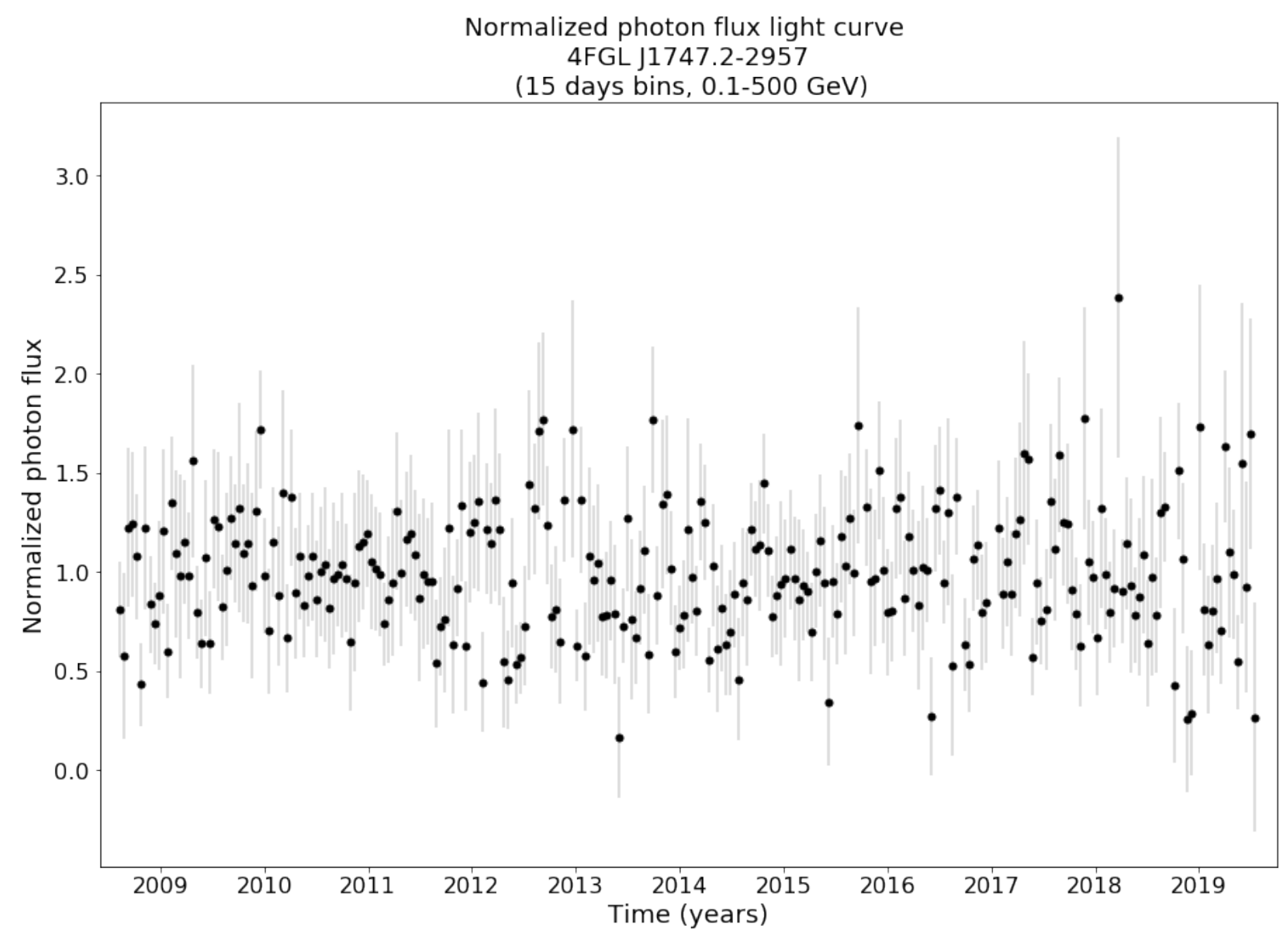

Figure I.1: 15 days average normalized photon flux LC of 4FGL J1747.2-2957 (pulsar PSR J1747-2958). This is a presumably steady sources and its flux variability is used to estimate the systematic uncertainty in the data. This LC was created following the exact same data selection and methods used to create 4FGL J1745.6-2859 LC. 


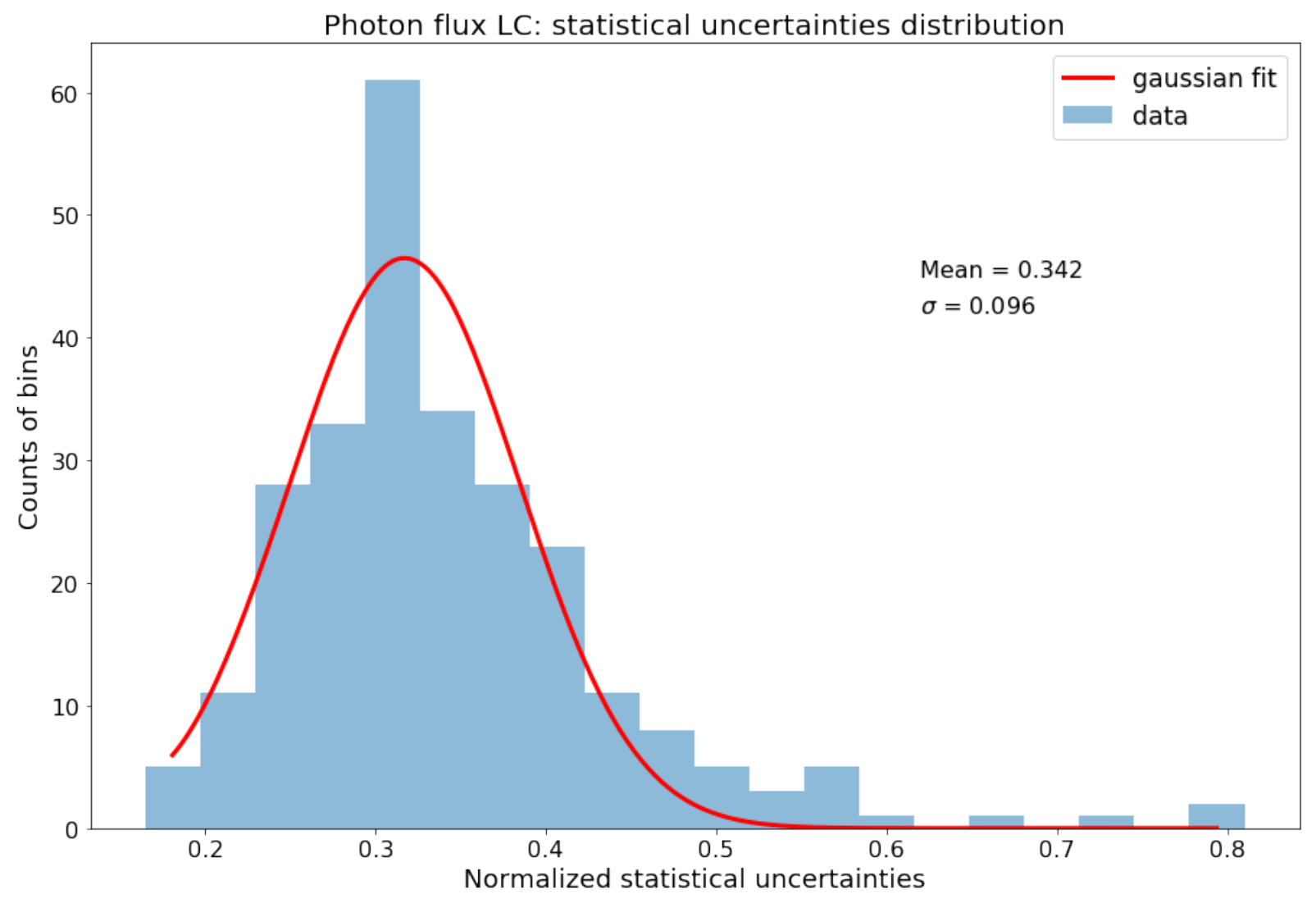

Figure I.2: Histogram of the statistical uncertainties of the 15 days average normalized photon flux LC of 4FGL J1747.2-2957 (Figure I.1). The dotted red line is a normal distribution with the same mean and standard deviation of the statistical uncertainties (the values are shown in the Figure). 


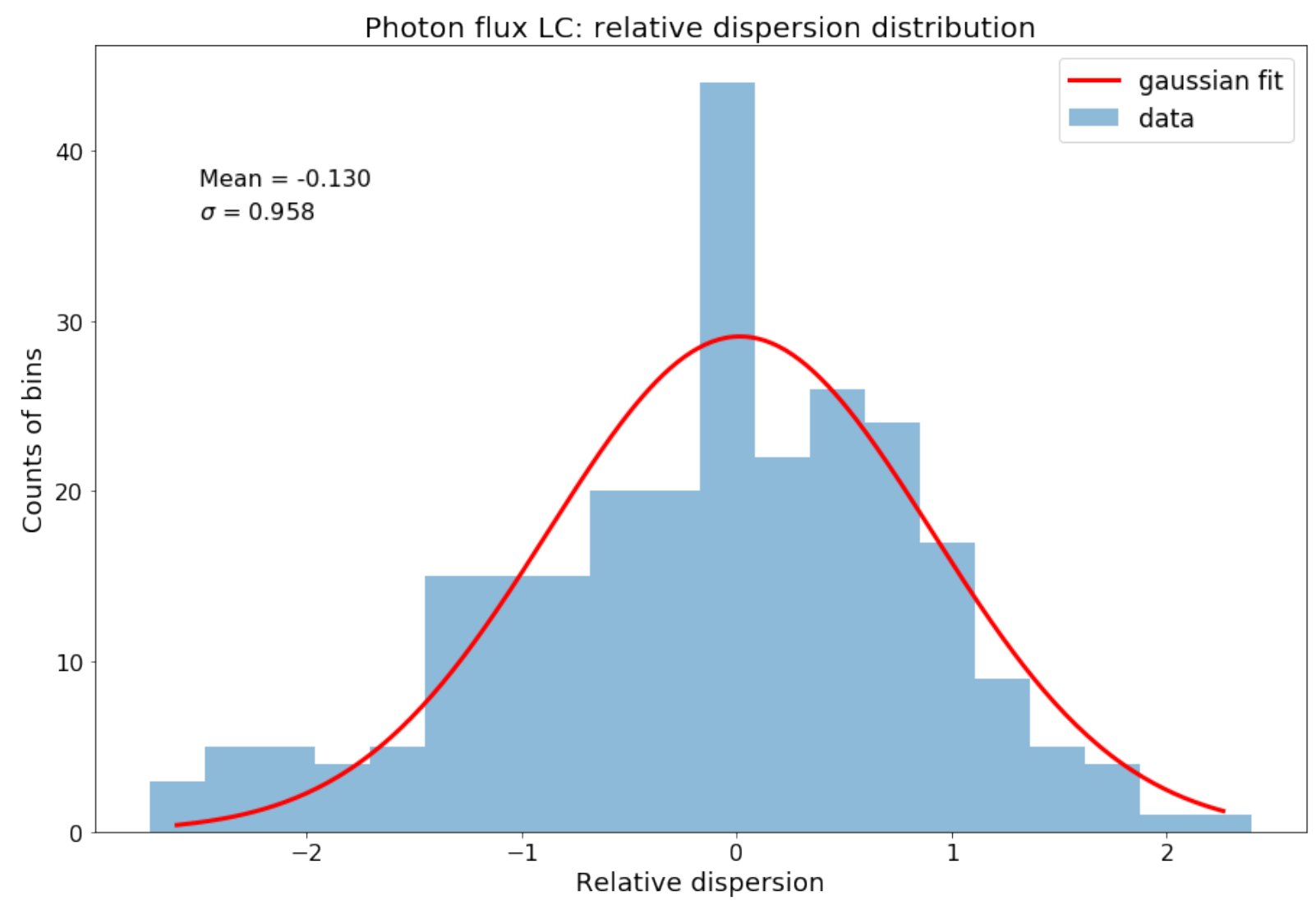

Figure I.3: Histogram of the relative dispersion distribution of the 15 days average normalized photon flux LC of 4FGL J1747.2-2957 (Figure I.1) calculated following Equation 2.13 The mean and standard deviation of this distribution are also shown in the Figure. 


\section{Appendix J}

\section{Estimating the systematic uncertainties in the LCs}

Here we perform the same analysis as in Section 2.5.2, but for the different LCs intervals and energy ranges, in order to estimate the systematic uncertainty in the data. We will only focus on showing the relevant plots, since the details of the analysis are in the main text.

\section{J.1 Systematic uncertainties in the 45 days $L C$}

Below, the systematic uncertainties estimate of the LCs in the $100 \mathrm{MeV}-500 \mathrm{GeV}$ energy range and with 45 days bins. 


\section{J.1.1 Energy flux}

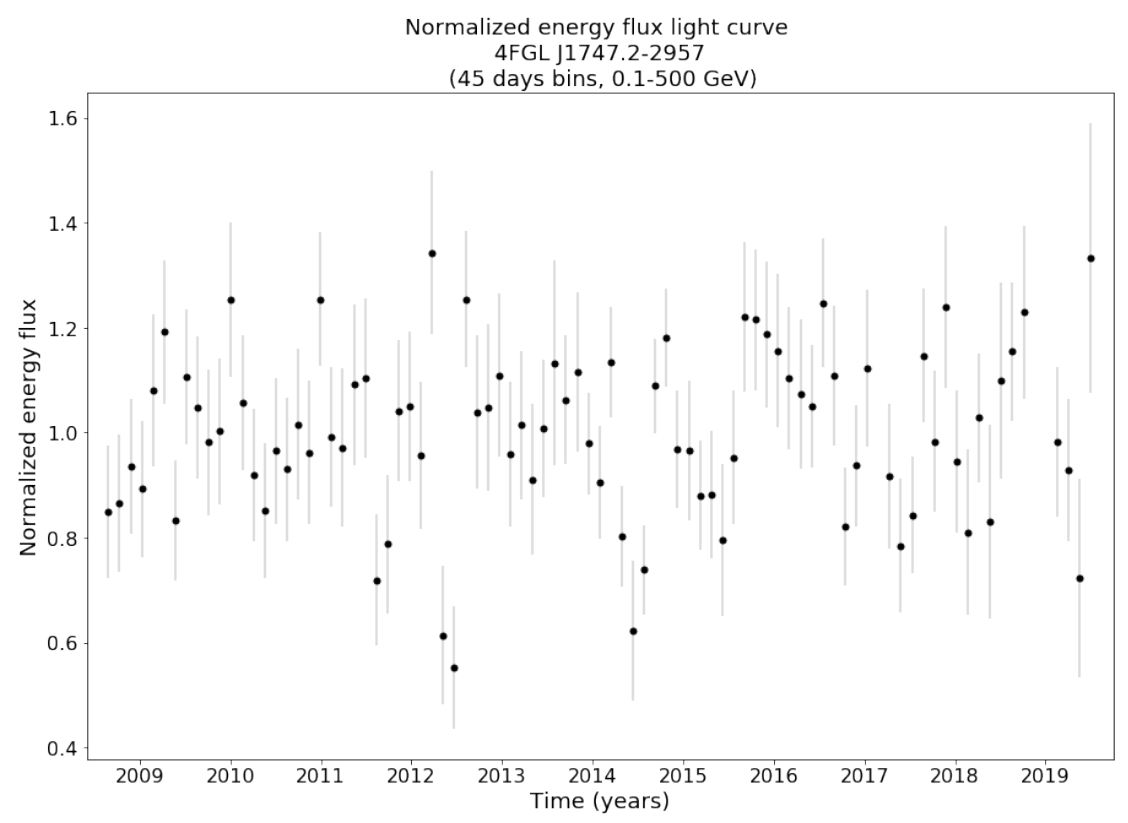

Figure J.1: 15 days average normalized energy flux LC of 4FGL J1747.2-2957 (pulsar PSR J1747-2958) in the $100 \mathrm{MeV}-500 \mathrm{GeV}$ energy range.

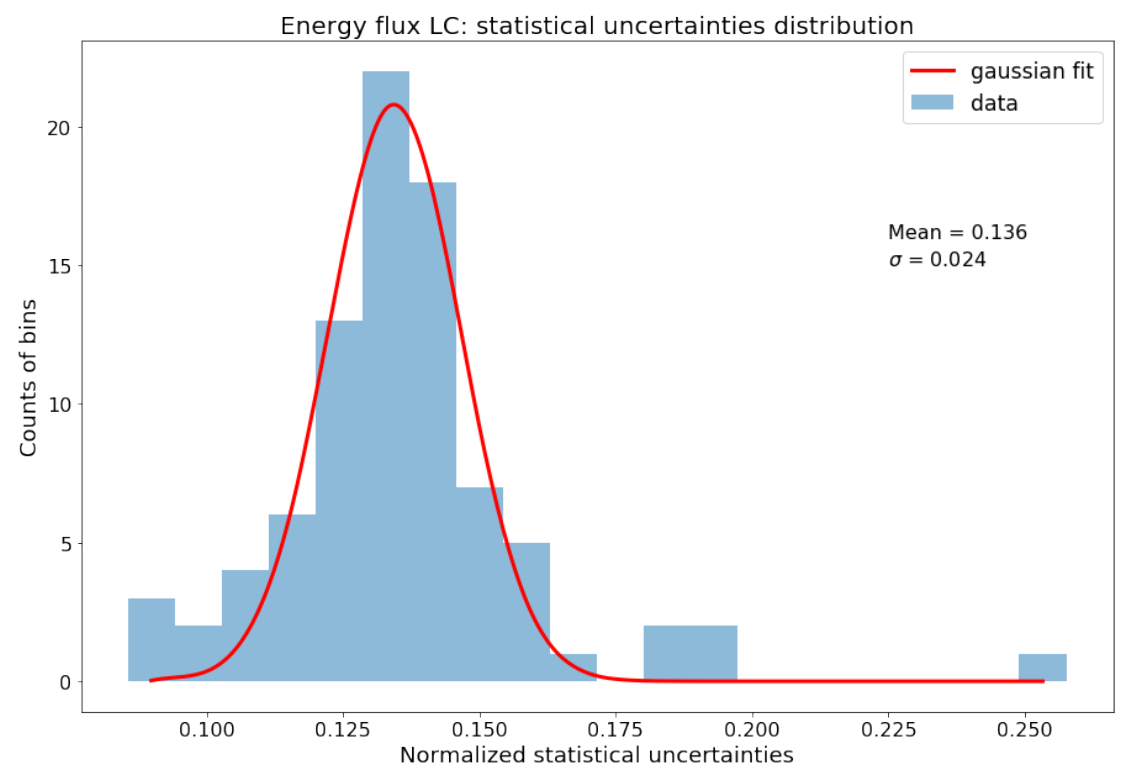

Figure J.2: Histogram of the statistical uncertainties of the 45 days average normalized energy flux LC of 4FGL J1747.2-2957 in the $100 \mathrm{MeV}-500 \mathrm{GeV}$ energy range. The red line is a normal distribution with the same mean and standard deviation of the statistical uncertainties (the values are shown in the Figure). 


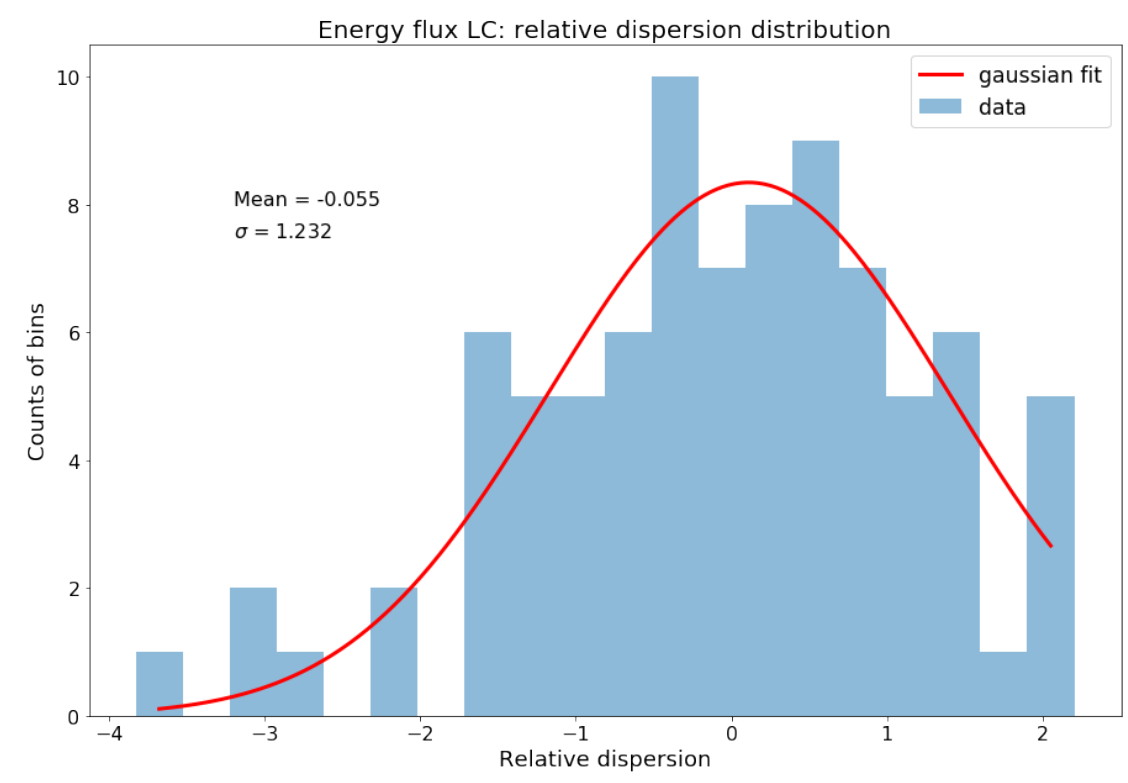

Figure J.3: Histogram of the relative dispersion distribution of the 45 days average normalized energy flux LC of 4FGL J1747.2-2957 in the $100 \mathrm{MeV}-500 \mathrm{GeV}$ energy range. The mean and standard deviation of this distribution are also shown in the Figure.

\section{J.1.2 Photon flux}

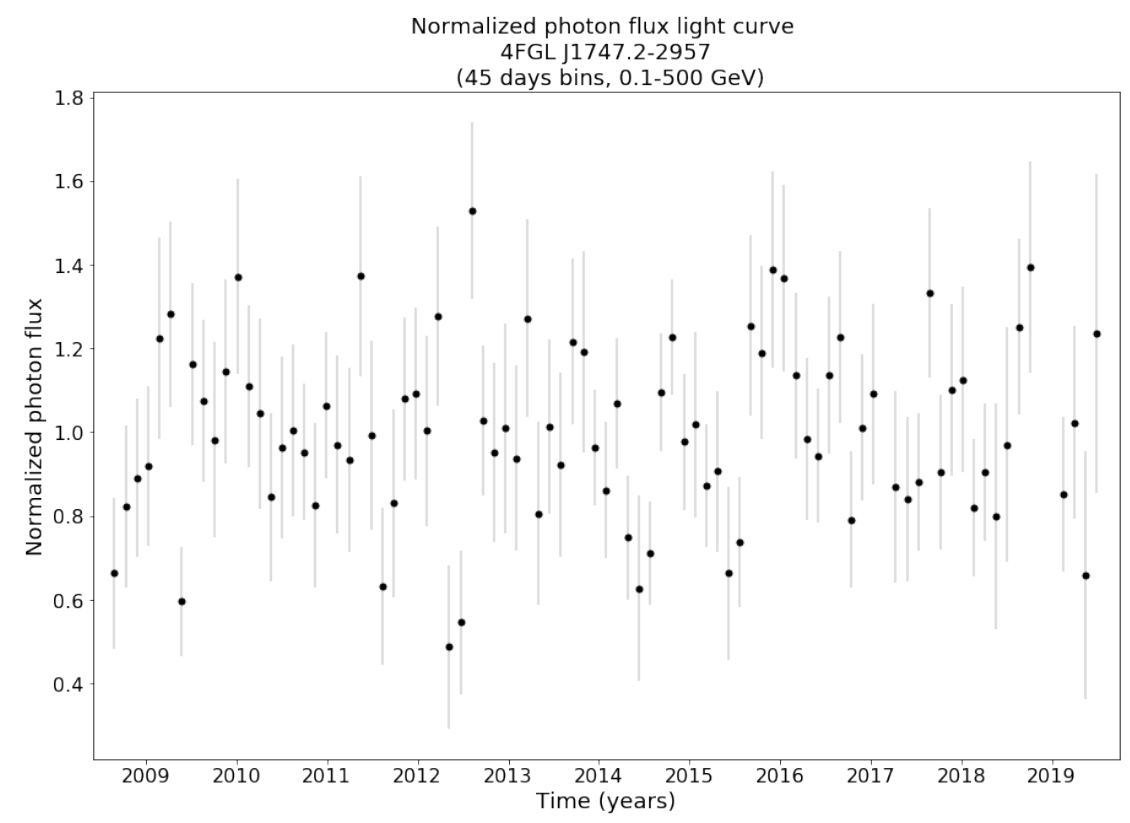

Figure J.4: 15 days average normalized photon flux LC of 4FGL J1747.2-2957 (pulsar PSR J1747-2958) in the $100 \mathrm{MeV}-500 \mathrm{GeV}$ energy range. 


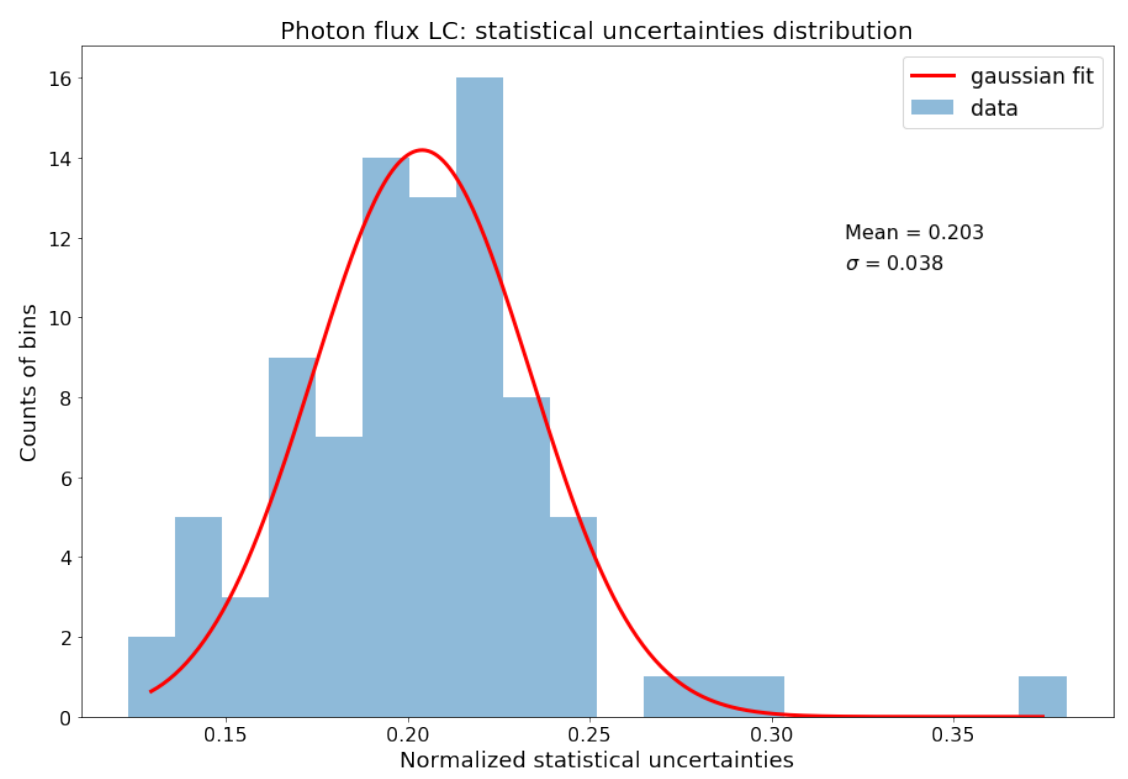

Figure J.5: Histogram of the statistical uncertainties of the 45 days average normalized photon flux LC of 4FGL J1747.2-2957 in the $100 \mathrm{MeV}-500 \mathrm{GeV}$ energy range. The red line is a normal distribution with the same mean and standard deviation of the statistical uncertainties (the values are shown in the Figure).

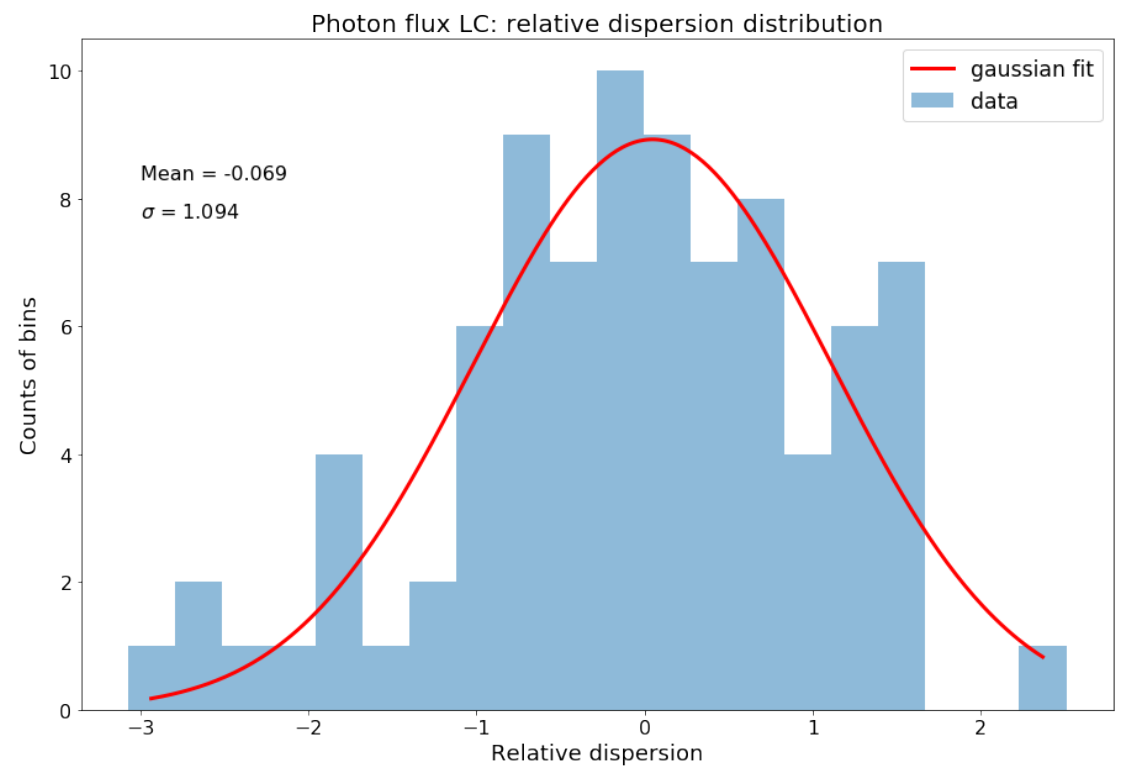

Figure J.6: Histogram of the relative dispersion distribution of the 45 days average normalized photon flux LC of 4FGL J1747.2-2957 in the $100 \mathrm{MeV}-500 \mathrm{GeV}$ energy range. The mean and standard deviation of this distribution are also shown in the Figure. 


\section{J.2 Systematic uncertainties in the 90 days, $300 \mathrm{MeV}-3 \mathrm{GeV} L C$}

Below, the systematic uncertainties estimate of the LCs in the $300 \mathrm{MeV}-3 \mathrm{GeV}$ energy range and with 90 days bins.

\section{J.2.1 Energy flux}

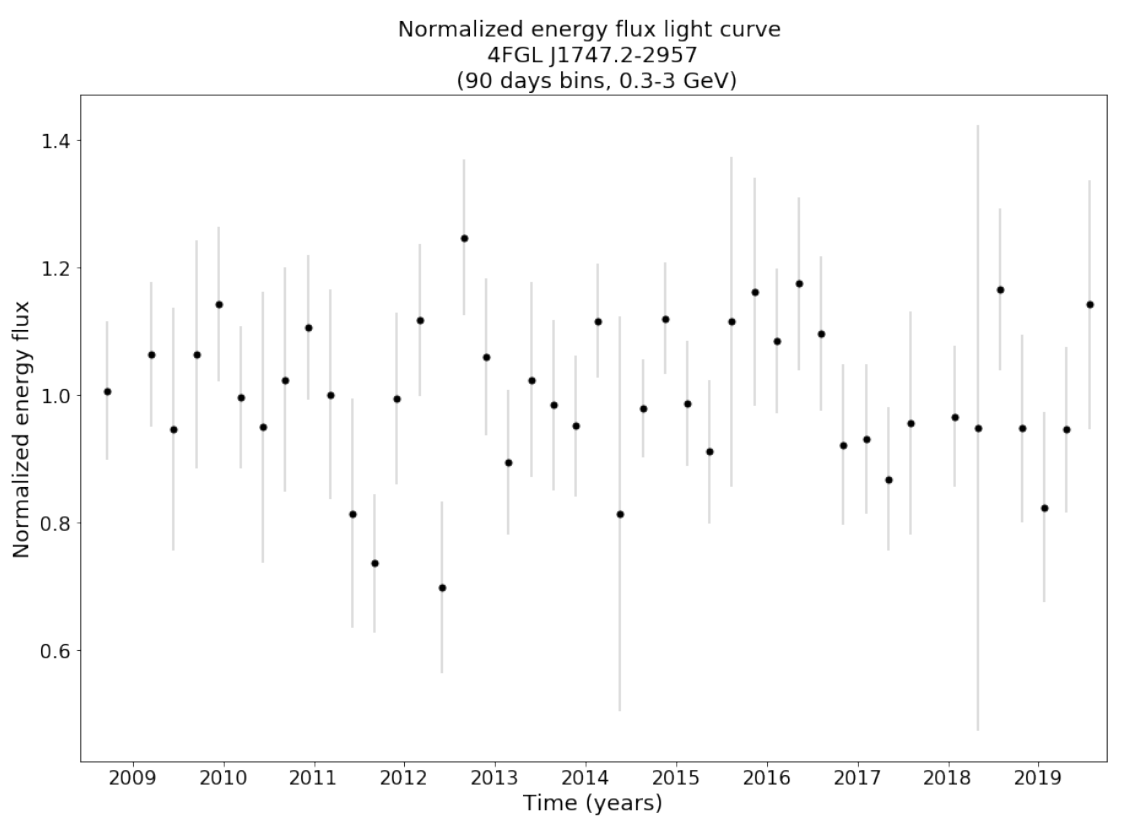

Figure J.7: 90 days average normalized energy flux LC of 4FGL J1747.2-2957 (pulsar PSR J1747-2958) in the $300 \mathrm{MeV}-3 \mathrm{GeV}$ energy range. 


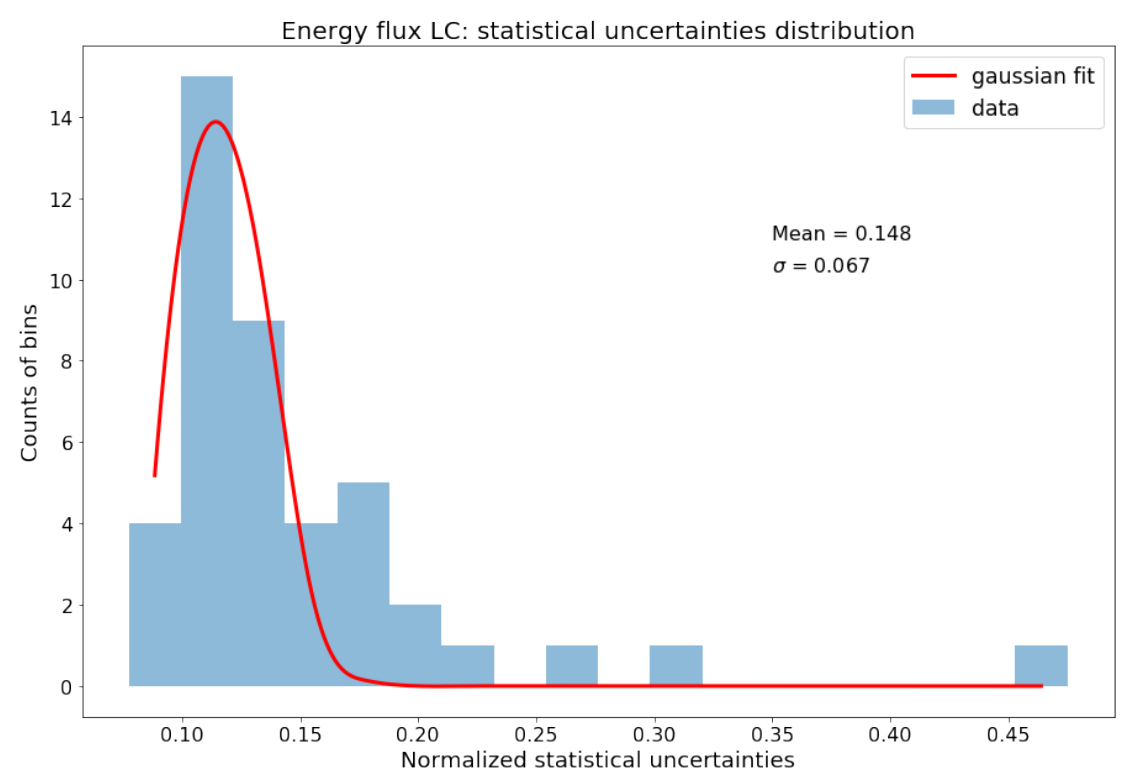

Figure J.8: Histogram of the statistical uncertainties of the 90 days average normalized energy flux LC of 4FGL J1747.2-2957 in the $300 \mathrm{MeV}-3 \mathrm{GeV}$ energy range. The red line is a normal distribution with the same mean and standard deviation of the statistical uncertainties (the values are shown in the Figure).

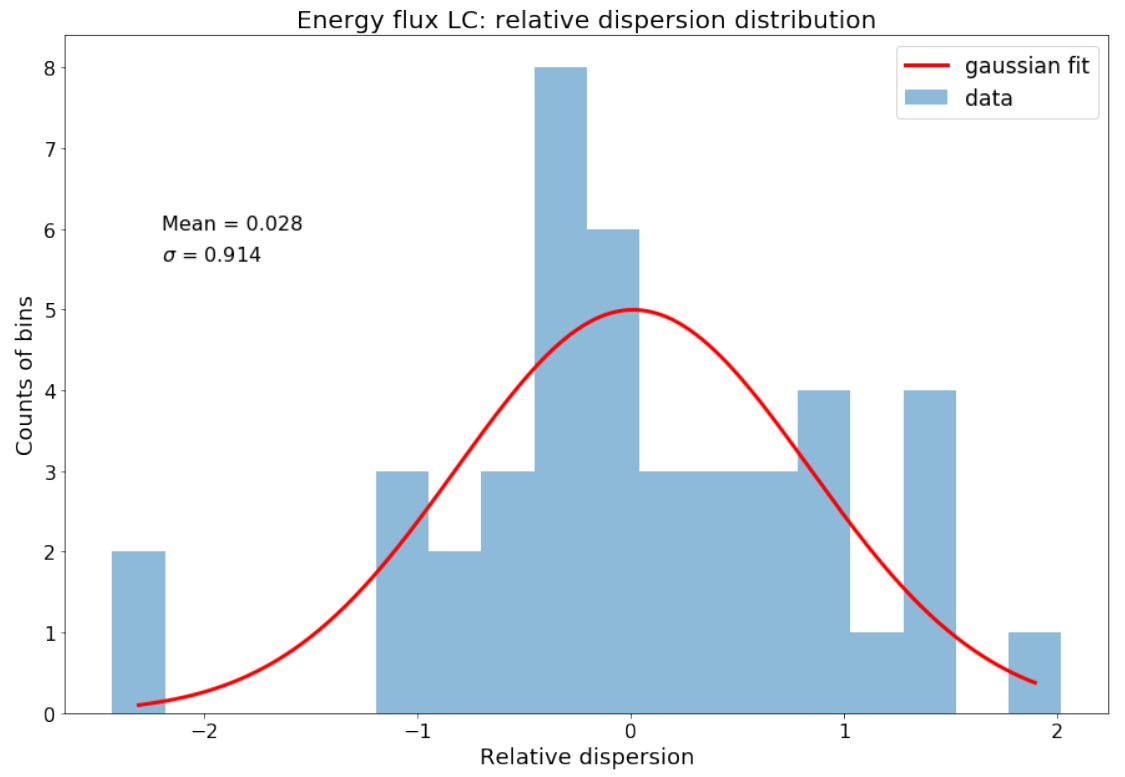

Figure J.9: Histogram of the relative dispersion distribution of the 90 days average normalized energy flux LC of 4FGL J1747.2-2957 in the $300 \mathrm{MeV}-3 \mathrm{GeV}$ energy range. The mean and standard deviation of this distribution are also shown in the Figure. 


\section{J.2.2 Photon flux}

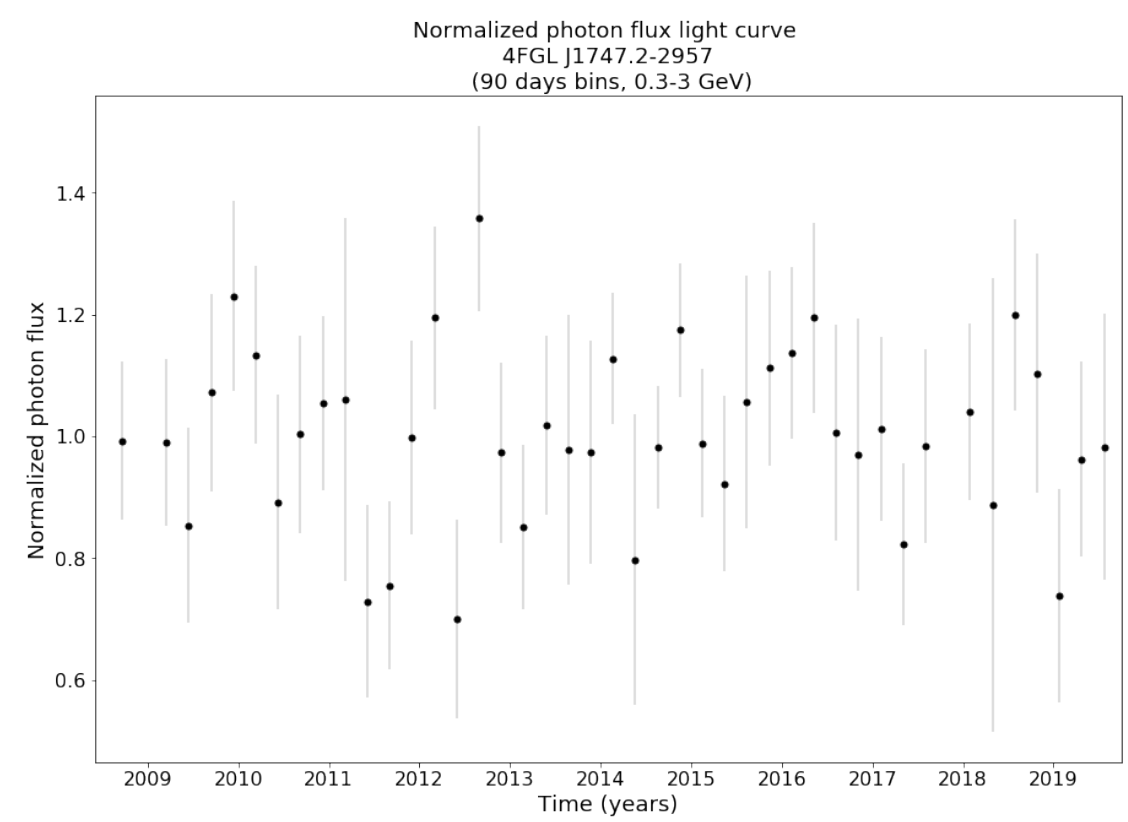

Figure J.10: 90 days average normalized photon flux LC of 4FGL J1747.2-2957 (pulsar PSR J1747-2958) in the $300 \mathrm{MeV}-3 \mathrm{GeV}$ energy range.

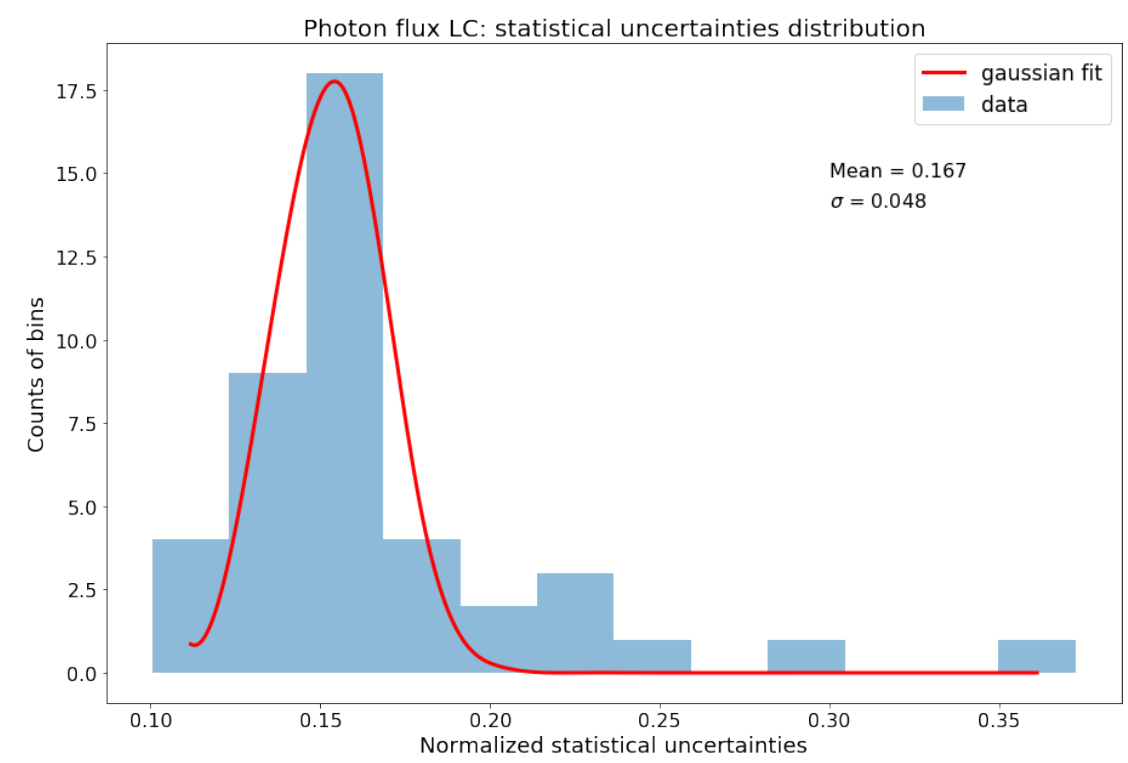

Figure J.11: Histogram of the statistical uncertainties of the 90 days average normalized photon flux LC of 4FGL J1747.2-2957 in the $300 \mathrm{MeV}-3 \mathrm{GeV}$ energy range. The red line is a normal distribution with the same mean and standard deviation of the statistical uncertainties (the values are shown in the Figure). 


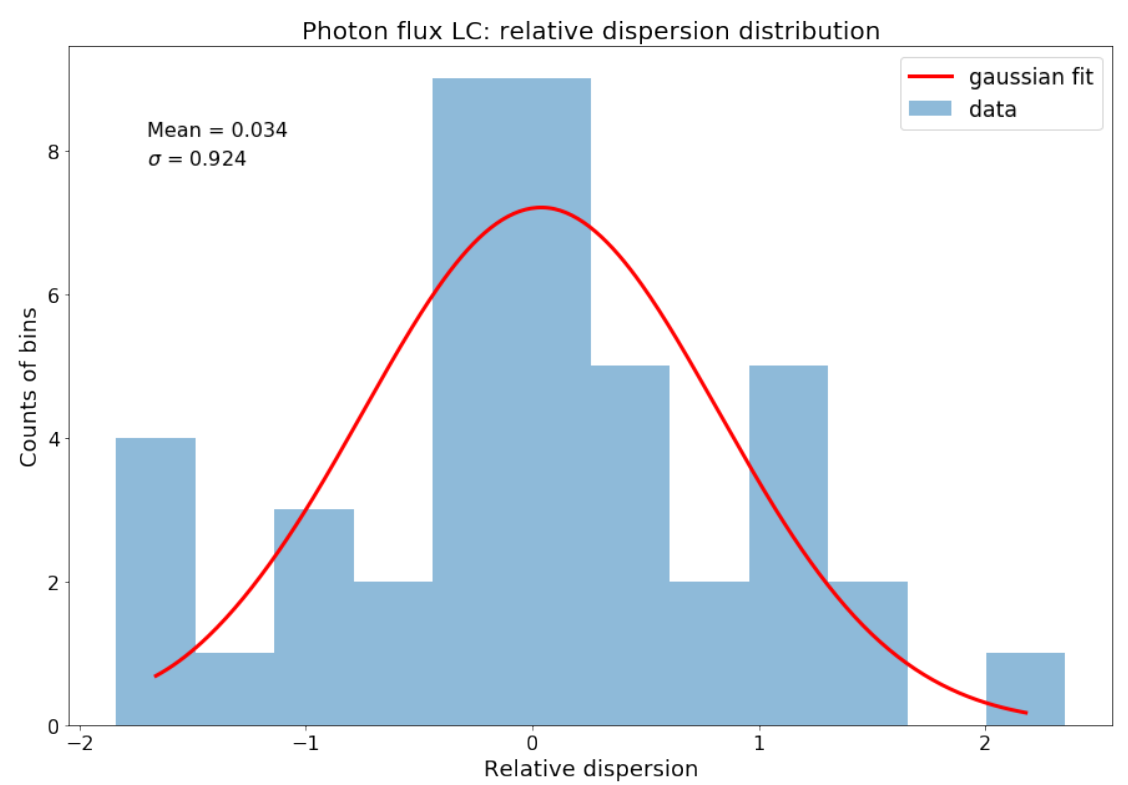

Figure J.12: Histogram of the relative dispersion distribution of the 90 days average normalized photon flux LC of 4FGL J1747.2-2957 in the $300 \mathrm{MeV}-3 \mathrm{GeV}$ energy range calculated following Equation 2.13 The mean and standard deviation of this distribution are also shown in the Figure.

\section{J.3 Systematic uncertainties in the 90 days, 3-10 GeV LC}

Below, the systematic uncertainties estimate of the LCs in the 3-10 GeV energy range and with 90 days bins. 


\section{J.3.1 Energy flux}

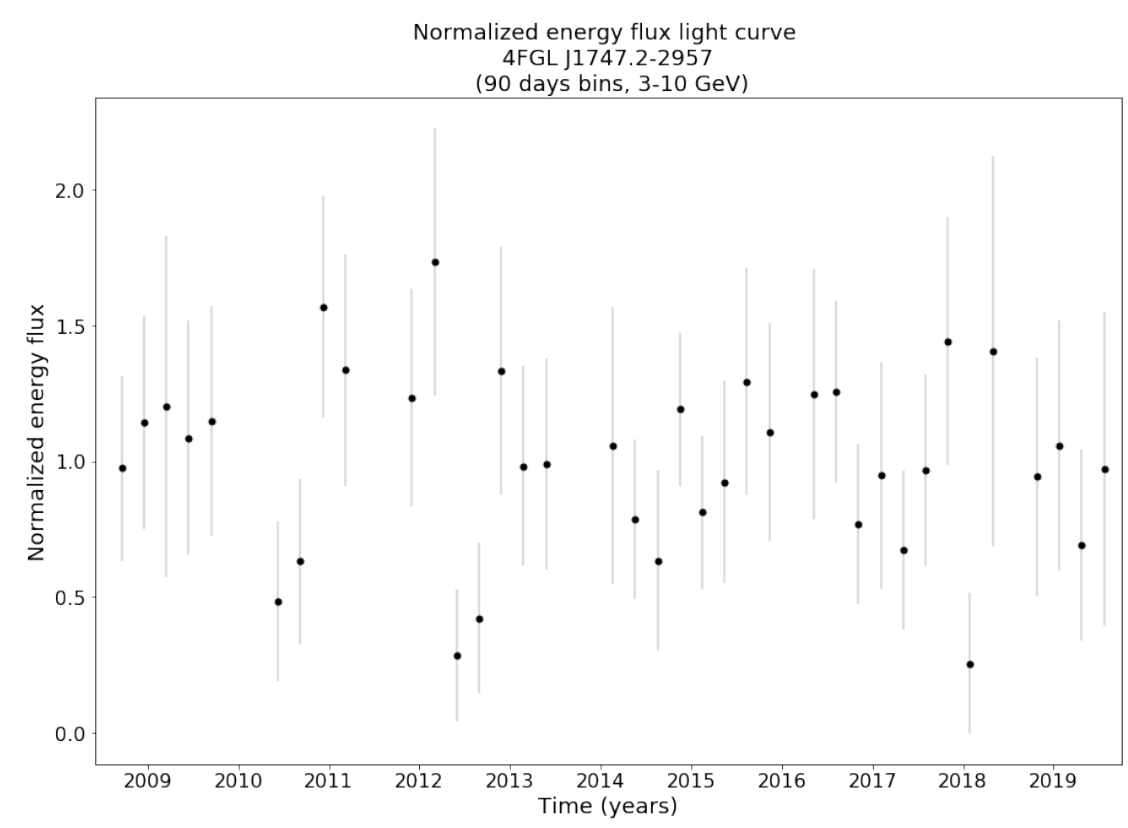

Figure J.13: 90 days average normalized energy flux LC of 4FGL J1747.2-2957 (pulsar PSR J1747-2958) in the $3-10 \mathrm{GeV}$ energy range.

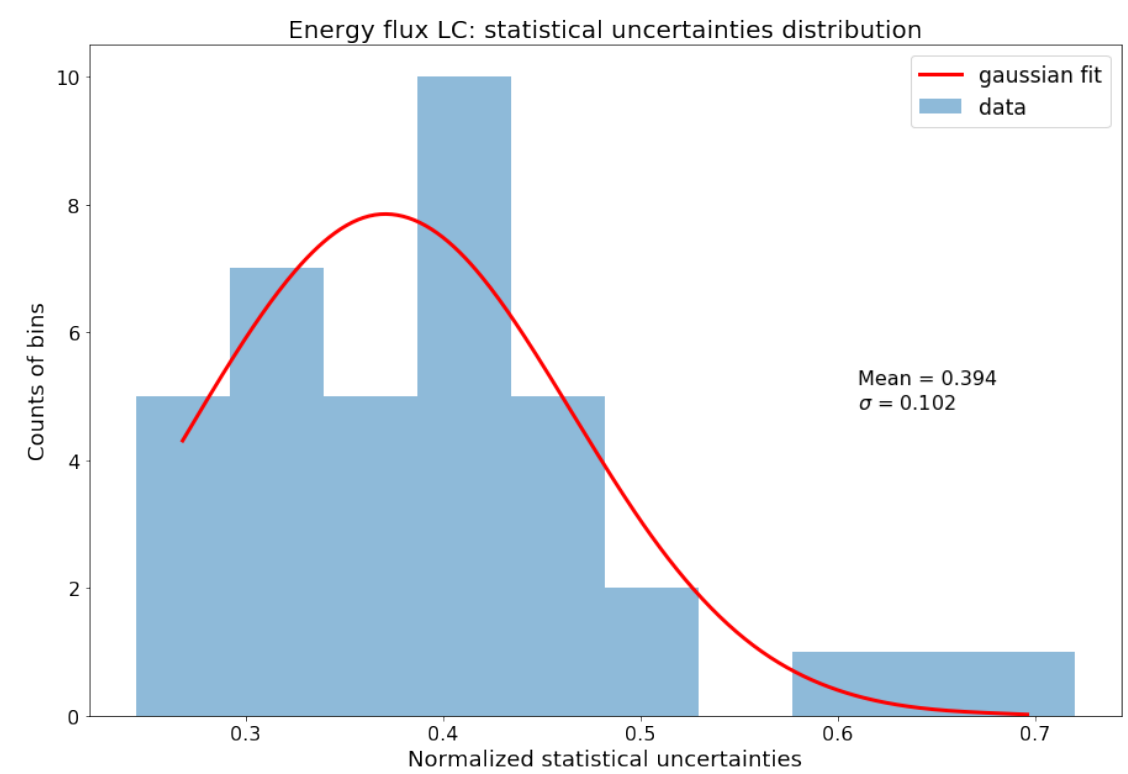

Figure J.14: Histogram of the statistical uncertainties of the 90 days average normalized energy flux LC of 4FGL J1747.2-2957 in the 3-10 GeV energy range. The red line is a normal distribution with the same mean and standard deviation of the statistical uncertainties (the values are shown in the Figure). 


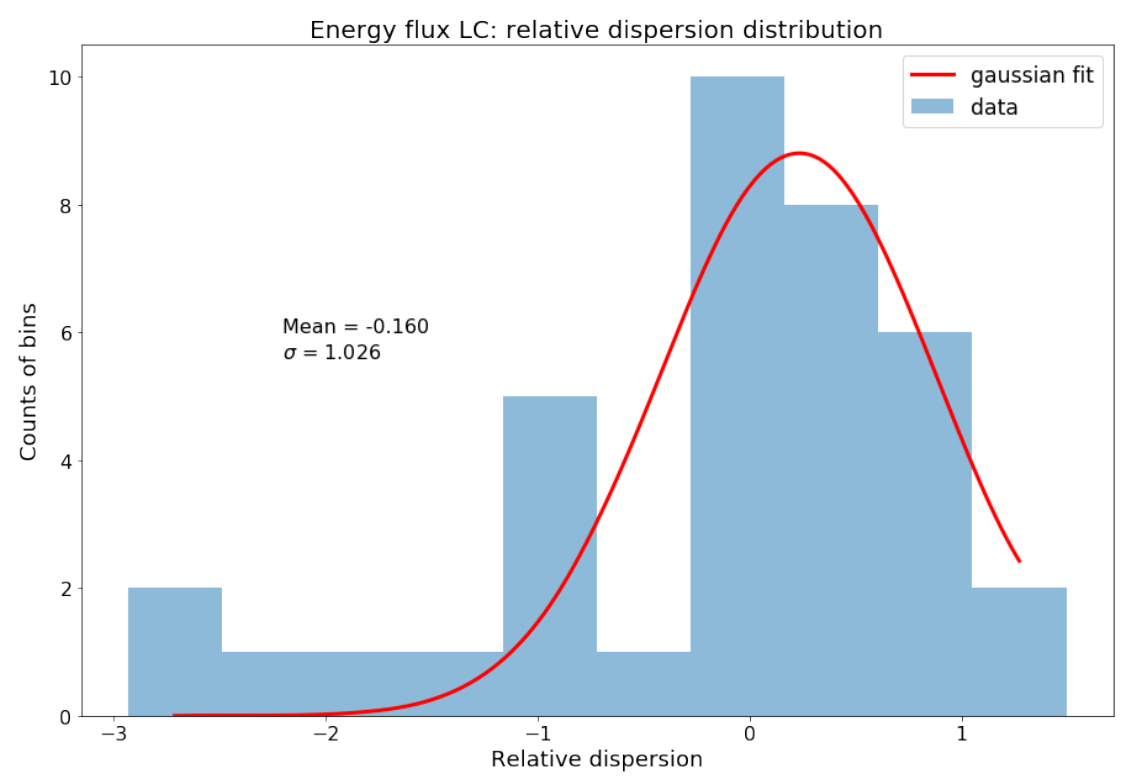

Figure J.15: Histogram of the relative dispersion distribution of the 90 days average normalized energy flux LC of 4FGL J1747.2-2957 in the 3-10 GeV energy range. The mean and standard deviation of this distribution are also shown in the Figure.

\section{J.3.2 Photon flux}

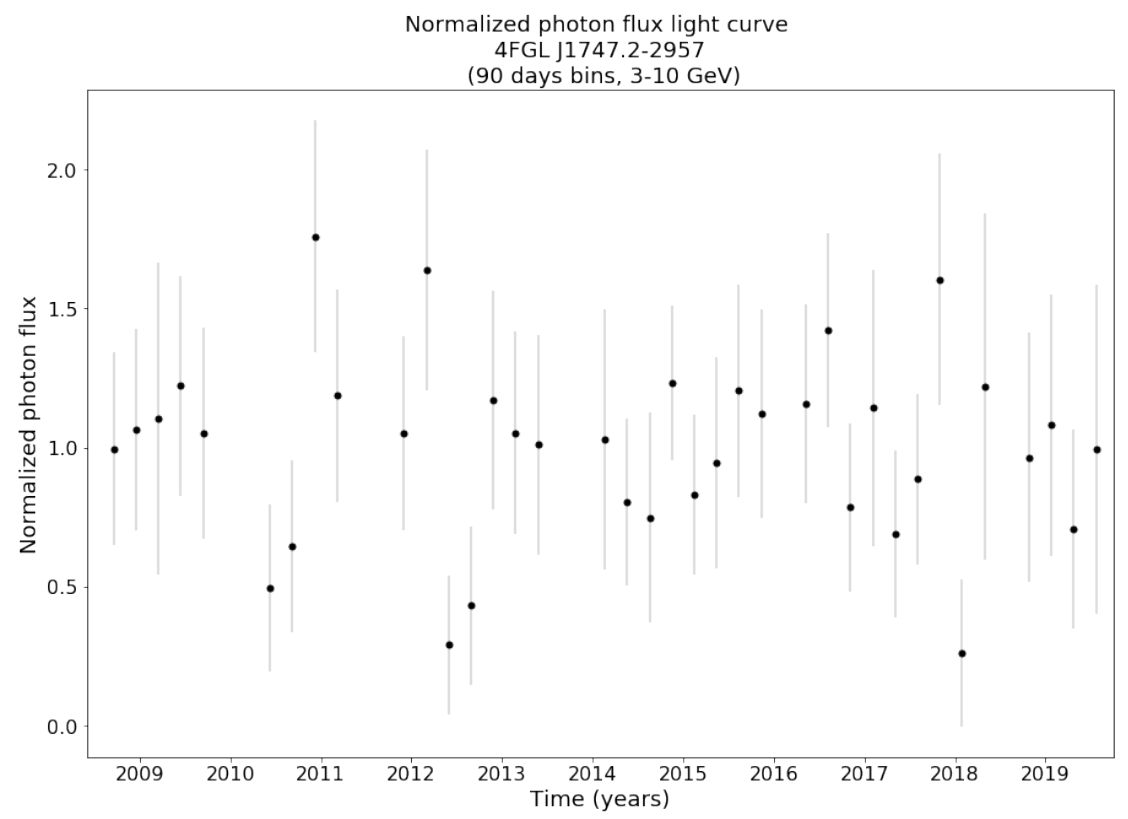

Figure J.16: 90 days average normalized photon flux LC of 4FGL J1747.2-2957 (pulsar PSR J1747-2958) in the 3-10 GeV energy range. 


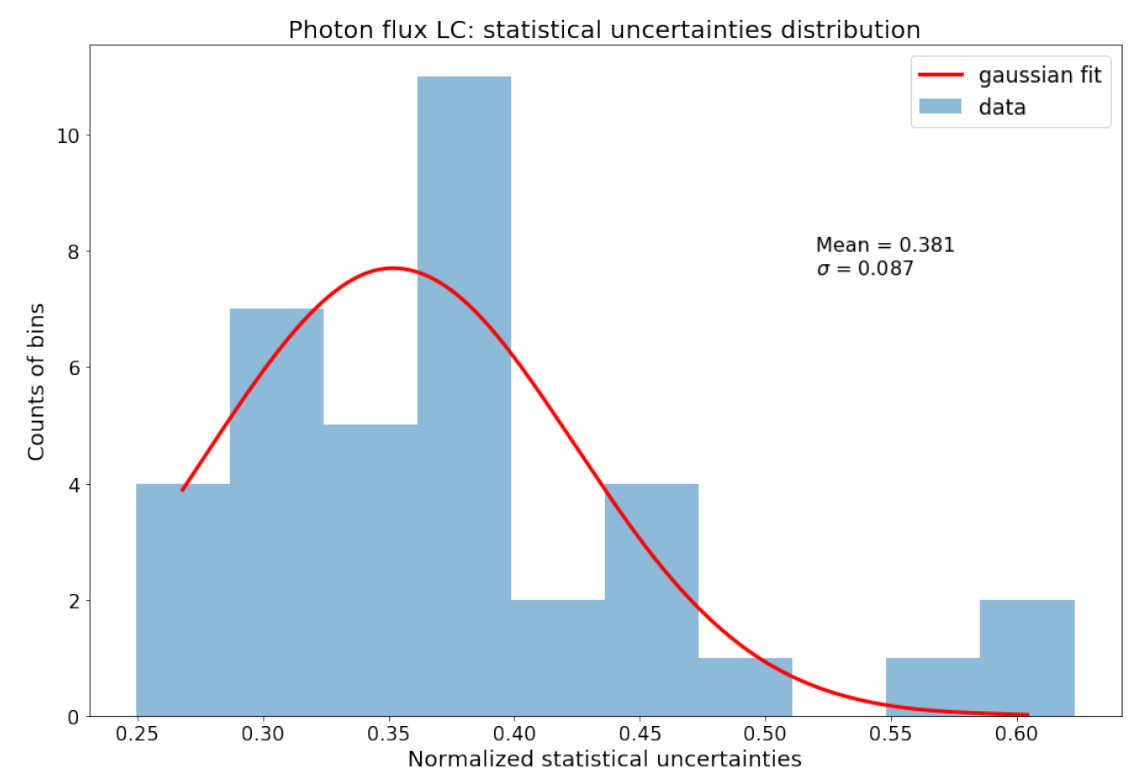

Figure J.17: Histogram of the statistical uncertainties of the 90 days average normalized photon flux LC of 4FGL J1747.2-2957 in the 3-10 GeV energy range. The red line is a normal distribution with the same mean and standard deviation of the statistical uncertainties (the values are shown in the Figure).

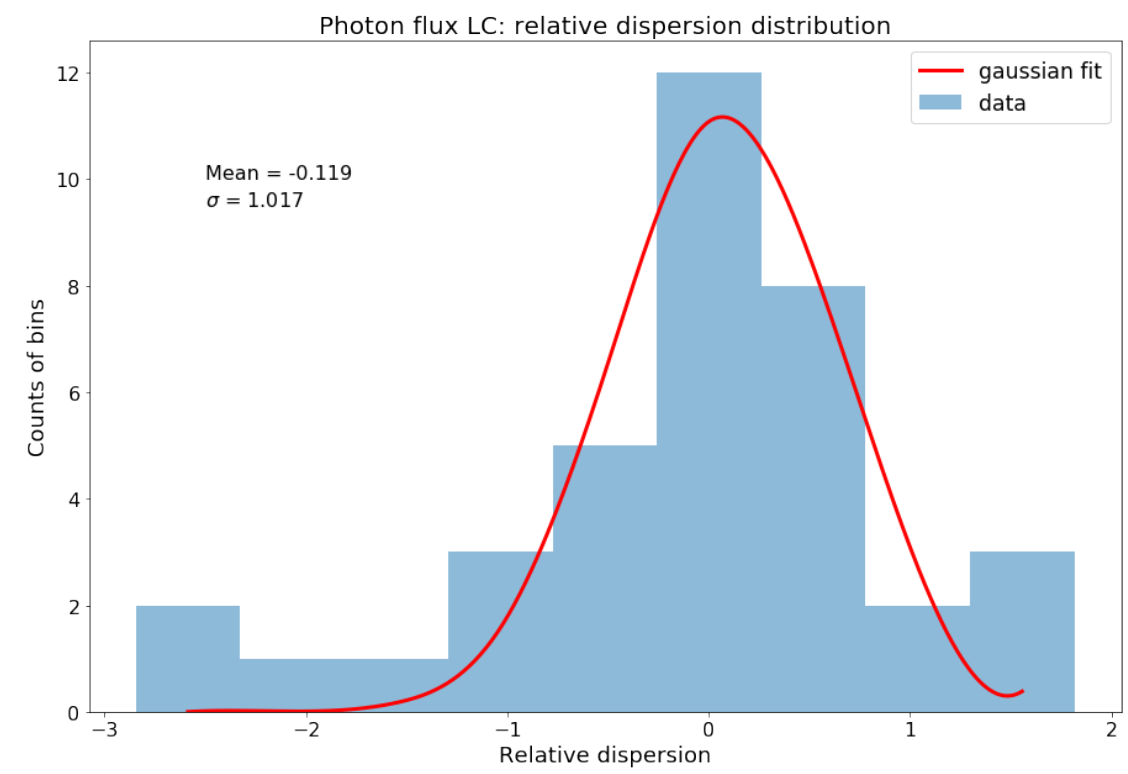

Figure J.18: Histogram of the relative dispersion distribution of the 90 days average normalized photon flux LC of 4FGL J1747.2-2957 in the 3-10 GeV energy range calculated following Equation 2.13. The mean and standard deviation of this distribution are also shown in the Figure. 


\section{Appendix K}

\section{The 45 days bins LC created based on the Universal Model $(100 \mathrm{MeV}-500 \mathrm{GeV})$}

Here we show the plots for the 45 days bins LC created based on the Universal Model (100 MeV-500 GeV energy range). They are similar to the plots shown in Section 3.3 for the 15 days bins LC in the same energy band.

The TS of the 45 days bins LC bins are shown in Appendix M. 

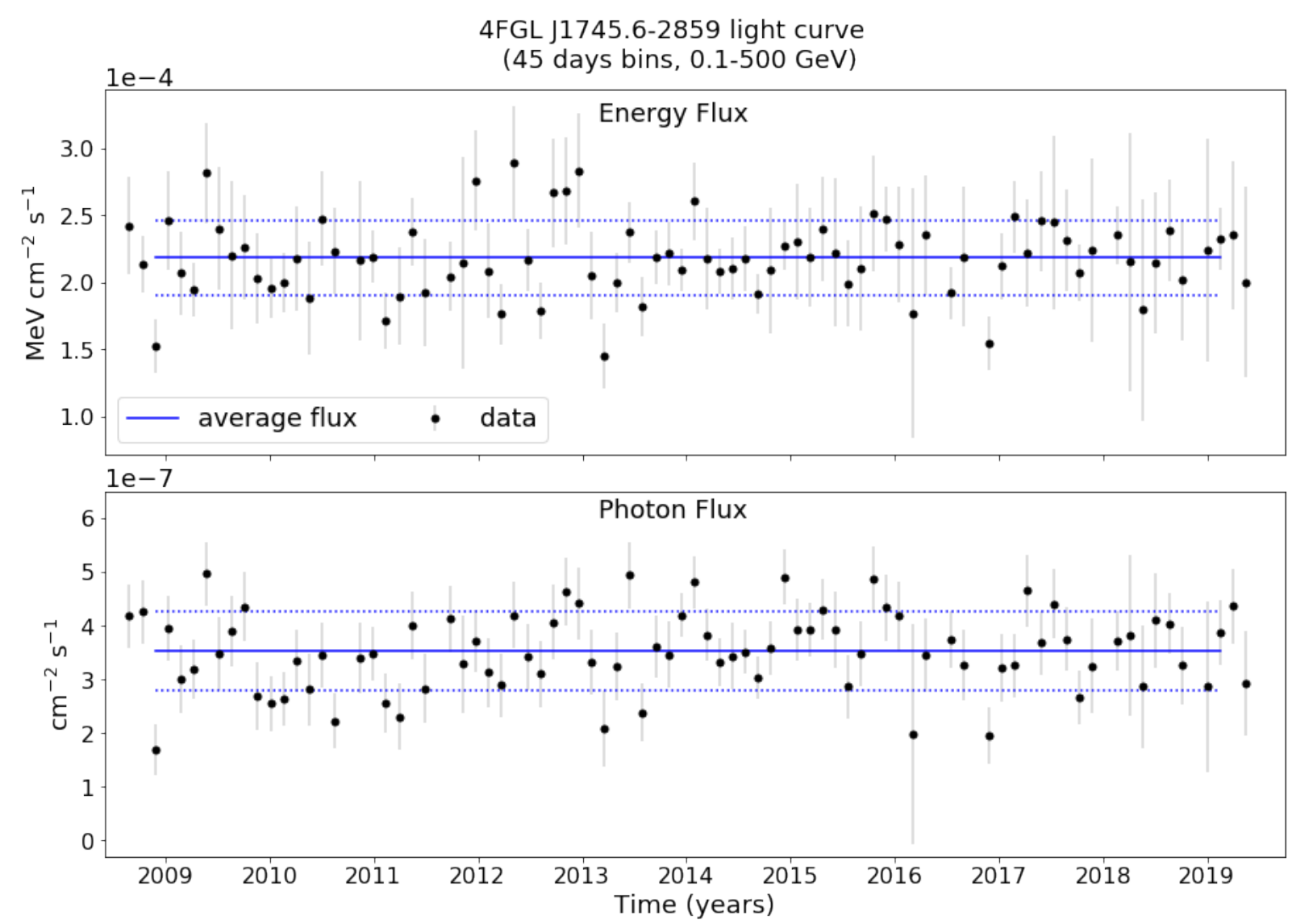

Figure K.1: 45 days LCs of 4FGL J1745.6-2859 in the $100 \mathrm{MeV}-500 \mathrm{GeV}$ energy band. The error bars correspond to the $68 \%$ confidence level uncertainty. In every bin the source was detected with TS $>16$. The dotted blue lines correspond to the $1 \sigma$ uncertainty of the average flux. 

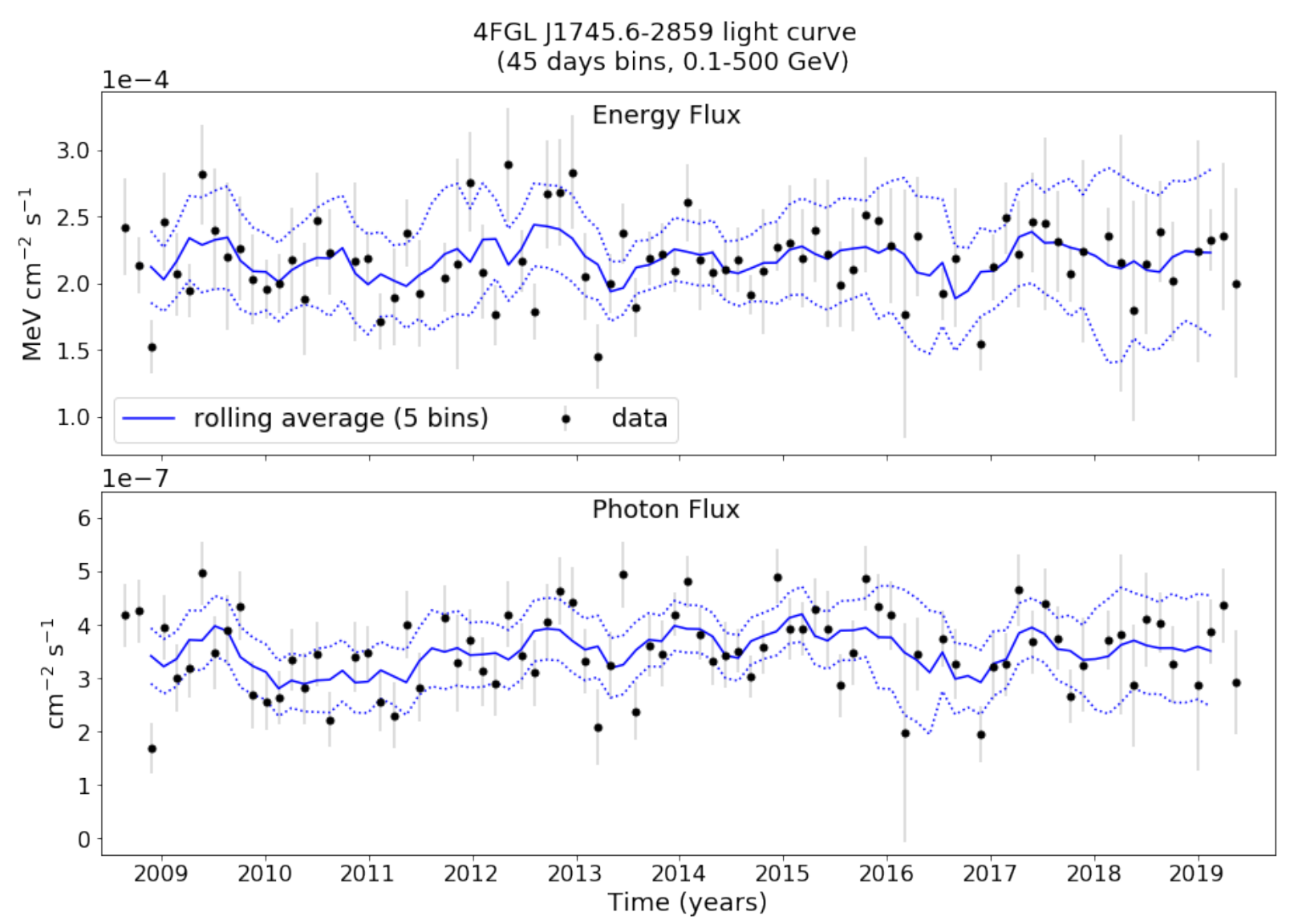

Figure K.2: 45 days LCs of 4FGL J1745.6-2859 in the $100 \mathrm{MeV}-500 \mathrm{GeV}$ energy band. The error bars correspond to the $68 \%$ confidence level uncertainty. The dotted lines correspond to the $2 \sigma$ uncertainty of the rolling average flux. 

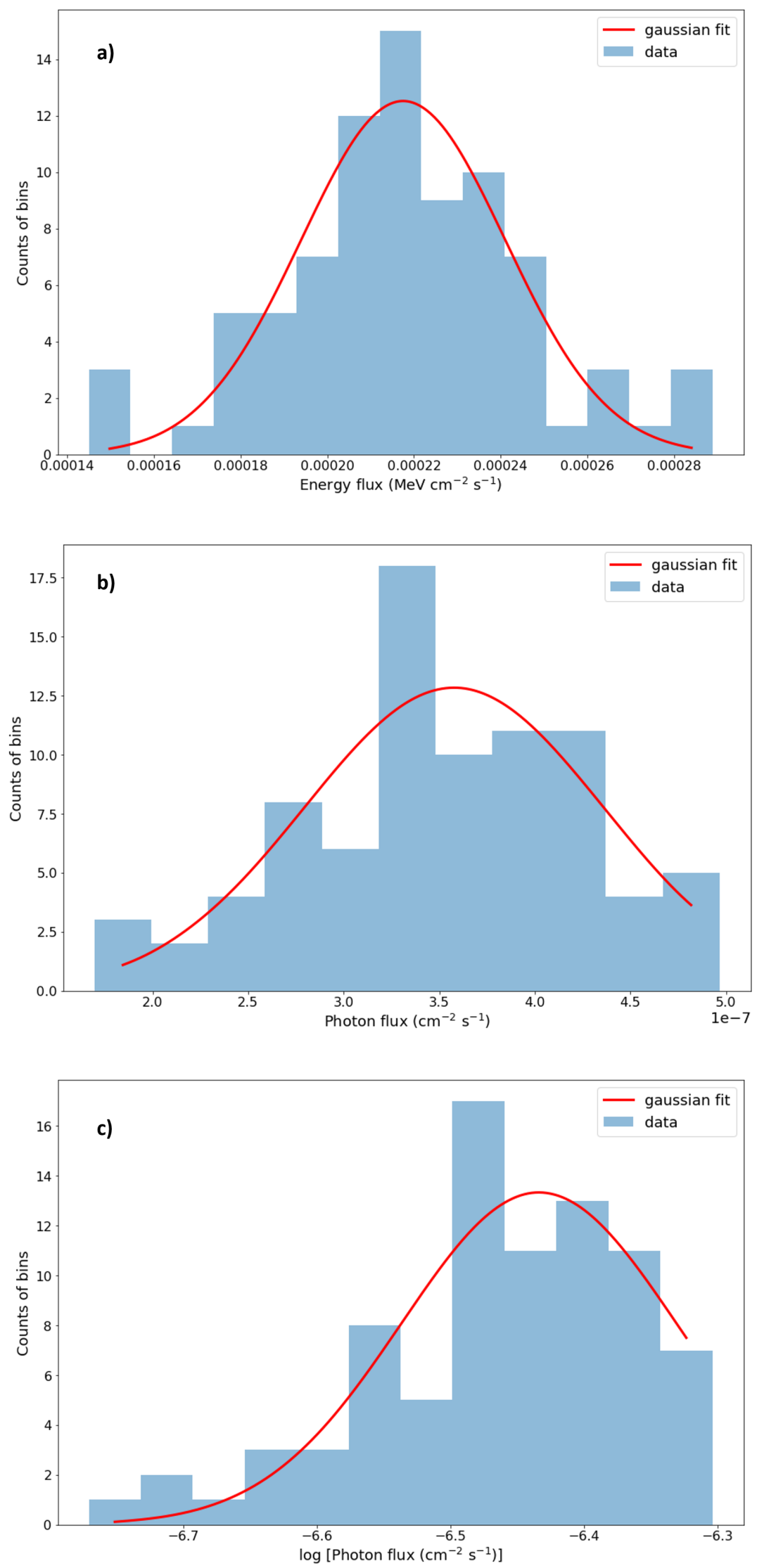

Figure K.3: The flux distribution for the 45 days bins LCs in the $0.1 \mathrm{MeV}-500 \mathrm{GeV}$. We show the distributions for the energy flux, the photon flux and the logarithm of the photon flux in panels a), b) and c), respectively. The red curve corresponds to the Gaussian fits to the distribution (a log-normal fit in panel c)). A Gaussian fit is clearly preferable for the fluxes distribution (see Tables K.1 and K.22. 
Table K.1 - Results of the Shapiro-Wilk and the D'Agostino's K ${ }^{2}$ normality tests for the 45 days LCs in the $0.1 \mathrm{MeV}-500 \mathrm{GeV}$ energy range. The flux distribution is compatible with a Gaussian fit.

\begin{tabular}{|c|c|c|c|c|c|c|}
\hline & \multicolumn{2}{|c|}{ Energy flux } & \multicolumn{2}{|c|}{ Photon flux } & \multicolumn{2}{|c|}{ Log(Photon flux) } \\
\hline & \multicolumn{2}{|c|}{$H_{0}$ : normal distribution } & \multicolumn{2}{|c|}{$H_{0}:$ normal distribution } & \multicolumn{2}{|c|}{$H_{0}$ : log-normal distributior } \\
\hline Test & p-value & result & p-value & result & p-value & result \\
\hline Shapiro-Wilk & 0.4615 & fail to reject $H_{0}$ & 0.6432 & fail to reject $H_{0}$ & 0.007 & reject $H_{0}$ \\
\hline D'Agostino's K ${ }^{2}$ & 0.562 & fail to reject $H_{0}$ & 0.684 & fail to reject $H_{0}$ & 0.009 & reject $H_{0}$ \\
\hline
\end{tabular}

Table K.2 - Results of the Anderson-Darling normality test for the 45 days LCs in the $0.1 \mathrm{MeV}-500 \mathrm{GeV}$ energy range. The flux distribution is better described by a Gaussian fit.

\begin{tabular}{|c|c|c|c|c|c|c|c|c|c|}
\hline & \multicolumn{3}{|c|}{ Energy flux } & \multicolumn{3}{|c|}{ Photon flux } & \multicolumn{3}{|c|}{ Log(Photon flux) } \\
\hline Sig. & \multicolumn{3}{|c|}{$H_{0}:$ normal distribution } & \multicolumn{3}{|c|}{$H_{0}:$ normal distribution } & \multicolumn{3}{|c|}{$H_{0}: \log$-normal distribution } \\
\hline level & statistic & crit. value & result & statistic & crit. value & result & statistic & crit. value & result \\
\hline 15 & & 0.551 & fail to reject $H_{0}$ & & 0.551 & fail to reject $H_{0}$ & & 0.551 & reject $H_{0}$ \\
\hline 10 & & 0.628 & fail to reject $H_{0}$ & & 0.628 & fail to reject $H_{0}$ & & 0.628 & reject $H_{0}$ \\
\hline 5 & 0.4178 & 0.753 & fail to reject $H_{0}$ & 0.1867 & 0.753 & fail to reject $H_{0}$ & 0.834 & 0.753 & reject $H_{0}$ \\
\hline 2.5 & & 0.878 & fail to reject $H_{0}$ & & 0.878 & fail to reject $H_{0}$ & & 0.878 & fail to reject $H_{0}$ \\
\hline 1 & & 1.045 & fail to reject $H_{0}$ & & 1.045 & fail to reject $H_{0}$ & & 1.045 & fail to reject $H_{0}$ \\
\hline
\end{tabular}



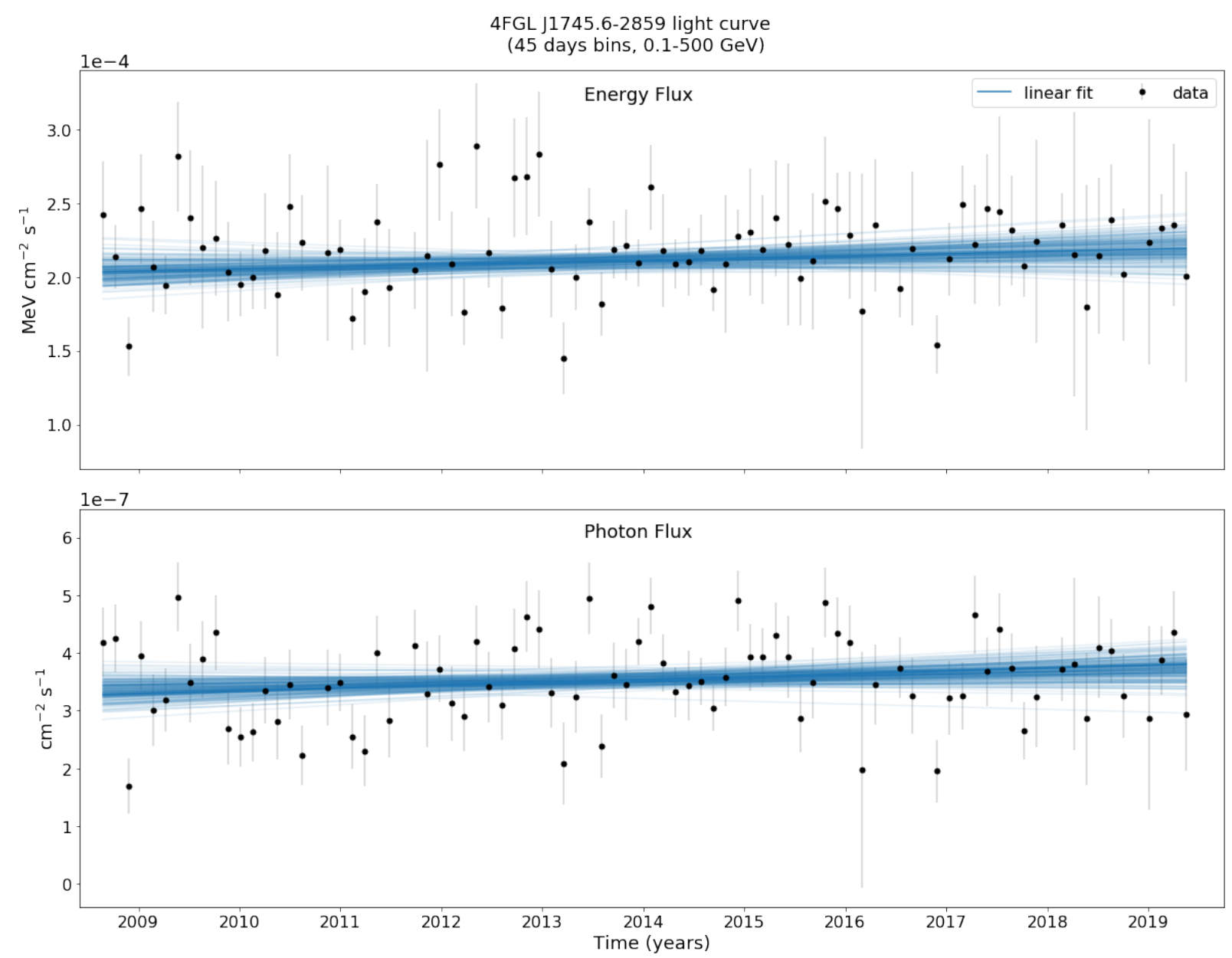

Figure K.4: These are the same 45 days LCs of 4FGL J1745.6-2859 in the $100 \mathrm{MeV}-500 \mathrm{GeV}$ energy band shown in Figure K.1 fitted with linear functions. We plotted in blue 200 randomized selected MCMC linear fits to the data. The results of the fit are in Table K.3

Table K.3 - Results of a linear fit to the $100 \mathrm{MeV}-500 \mathrm{GeV}$ energy range 45 days bins LCs.

\begin{tabular}{ccc}
\hline \hline Parameter & \multicolumn{2}{c}{ Light curve } \\
& Energy flux & Photon flux \\
\hline Angular coefficient & $(4.2 \pm 4.2) \times 10^{-14}$ & $(12.9 \pm 9.0) \times 10^{-17}$ \\
Linear coefficient & $(19.2 \pm 1.7) \times 10^{-5}$ & $(30.2 \pm 3.7) \times 10^{-8}$ \\
\hline
\end{tabular}


Appendix L

\section{The $95 \%$ confidence level central point position as a function of energy}

Figure L.1 shows the dependence of the source location on energy, together with the radio position of Sgr A* as measured by the Very Long Baseline Array (Petrov et al., 2011) and locations of other potential gamma-ray emitters in the GC. The solid and dashed circles represent the $68 \%$ and $95 \%$ confidence level positional uncertainties. 


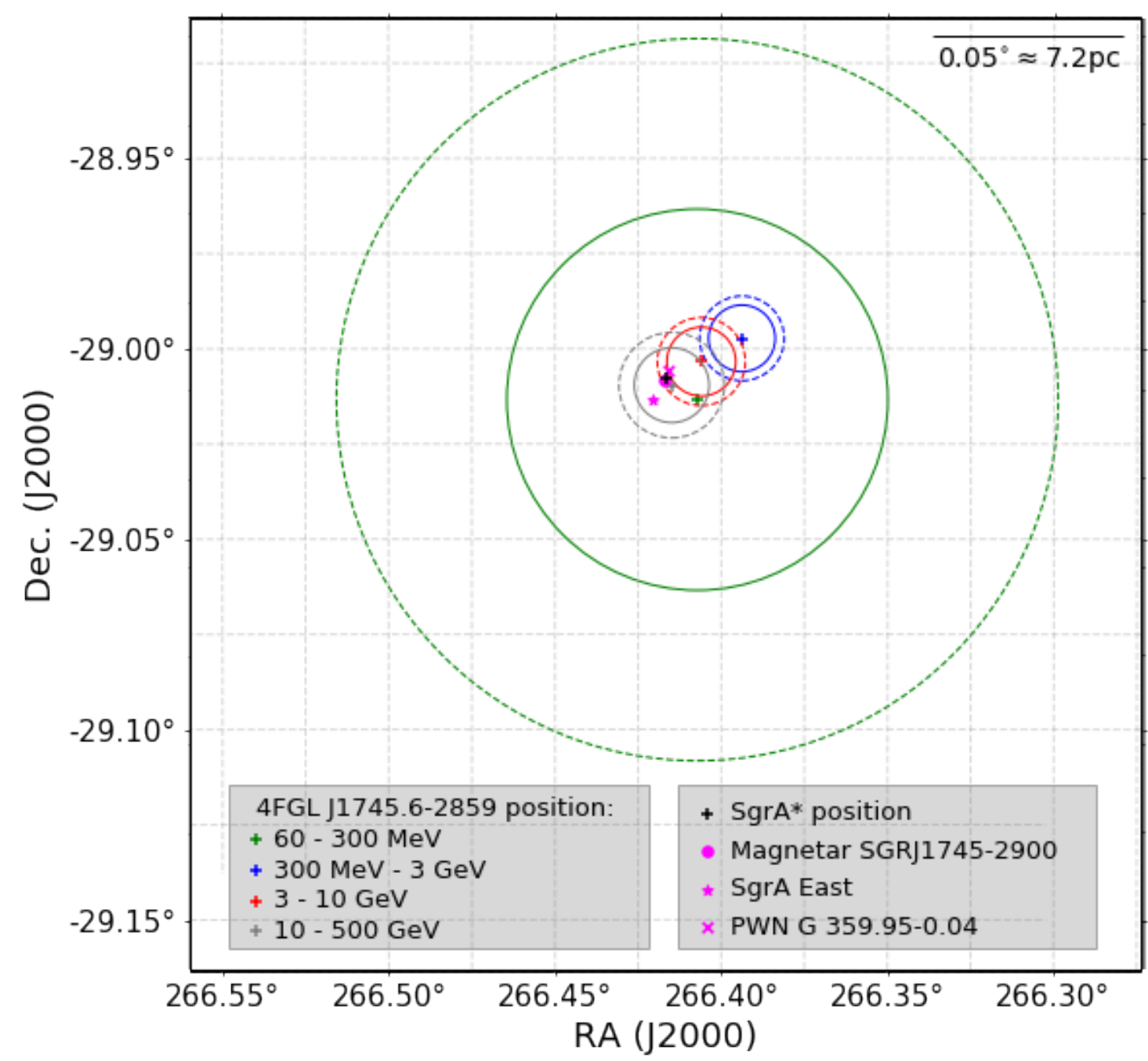

Figure L.1: The position of the central source as a function of the energy range used in the analysis: green (60-300 MeV), blue $(300 \mathrm{MeV}-3 \mathrm{GeV})$, red $(3-10 \mathrm{GeV})$ and gray $(10-500 \mathrm{GeV})$. The solid and dashed circles represent the $68 \%$ and $95 \%$ confidence level positional uncertainties. The radio position of Sgr A* is indicated by the black cross. The positions of other gamma-ray-emitters in the GC are also indicated. 


\section{Appendix M}

\section{The TS of the LCs}

Here we present the $T S$ of every bin of the LCs created in this work. For the bins with $T S<16$, indicated by a red line in the Figures, we showed the $95 \%$ confidence level UL in the LCs.

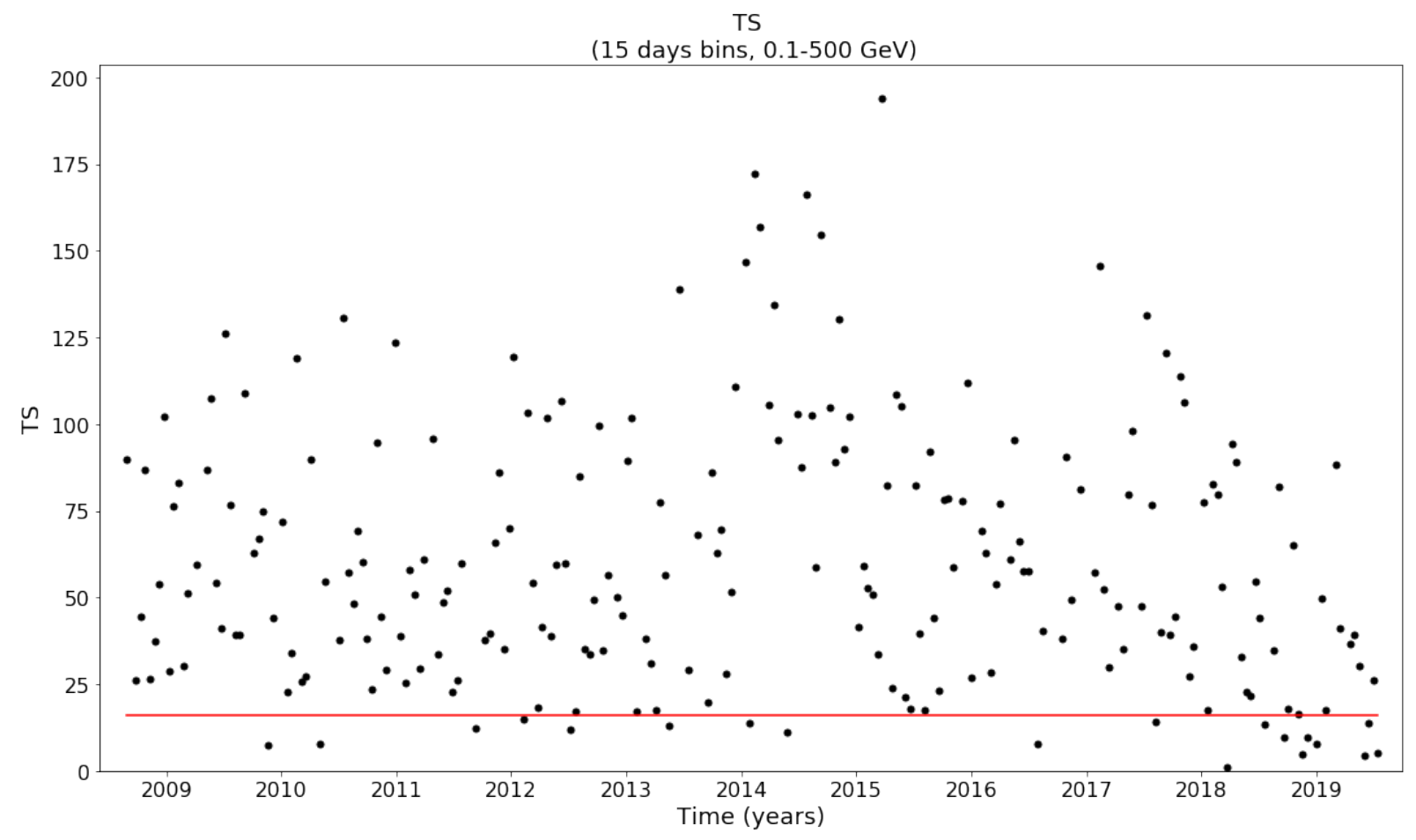

Figure M.1: The TS of the 15 days LC of 4FGL J1745.6-2859 in the $100 \mathrm{MeV}-500 \mathrm{GeV}$ energy band. A red line indicates the limit of $T S=16$ under which we showed the $95 \%$ confidence level UL in the LCs in this work. 
TS

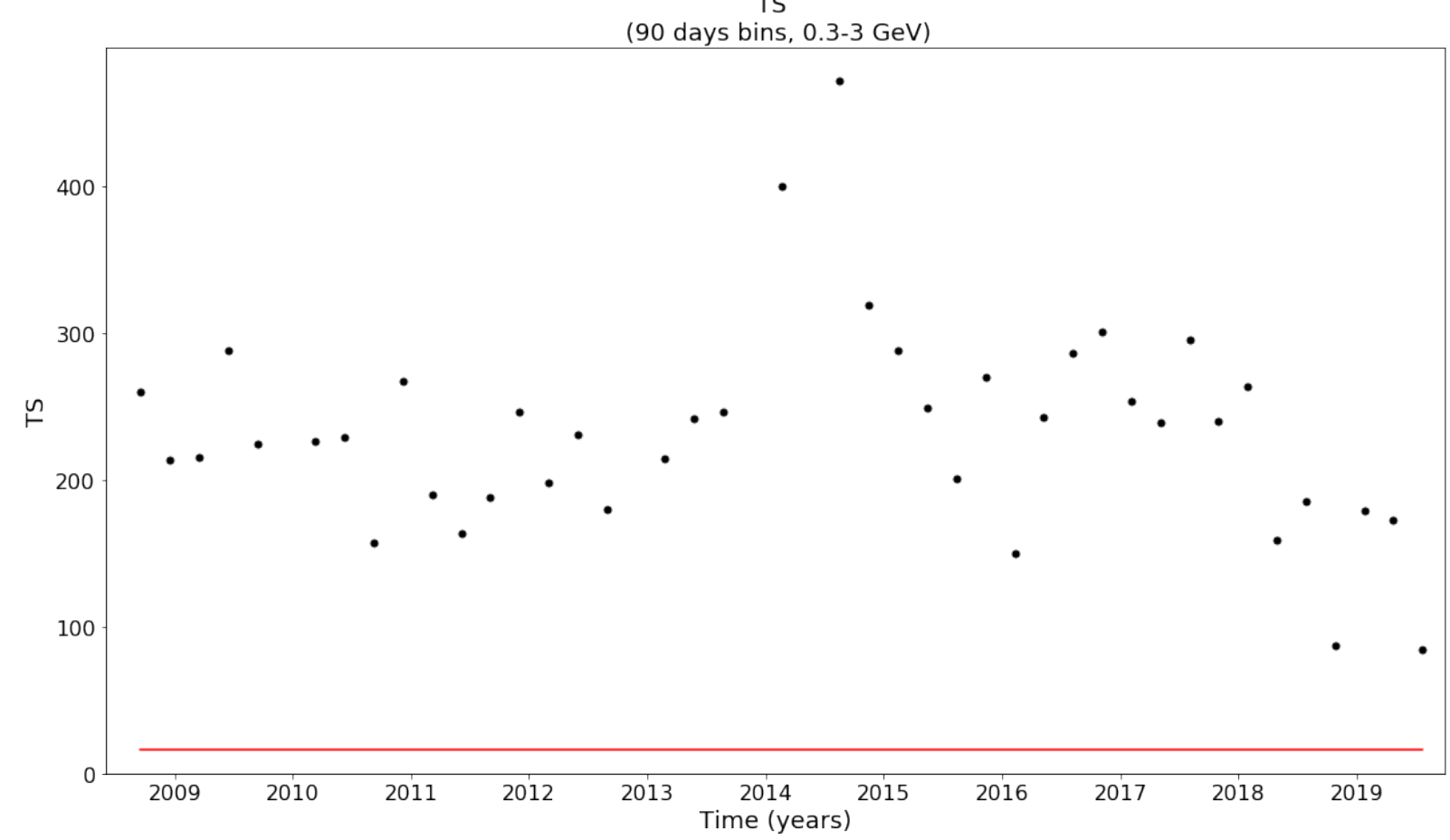

Figure M.2: The TS of the 90 days LC of 4FGL J1745.6-2859 in the $300 \mathrm{MeV}-3 \mathrm{GeV}$ energy band. The red line indicate the limit of $T S=16$.

TS

(90 days bins, 3-10 GeV)

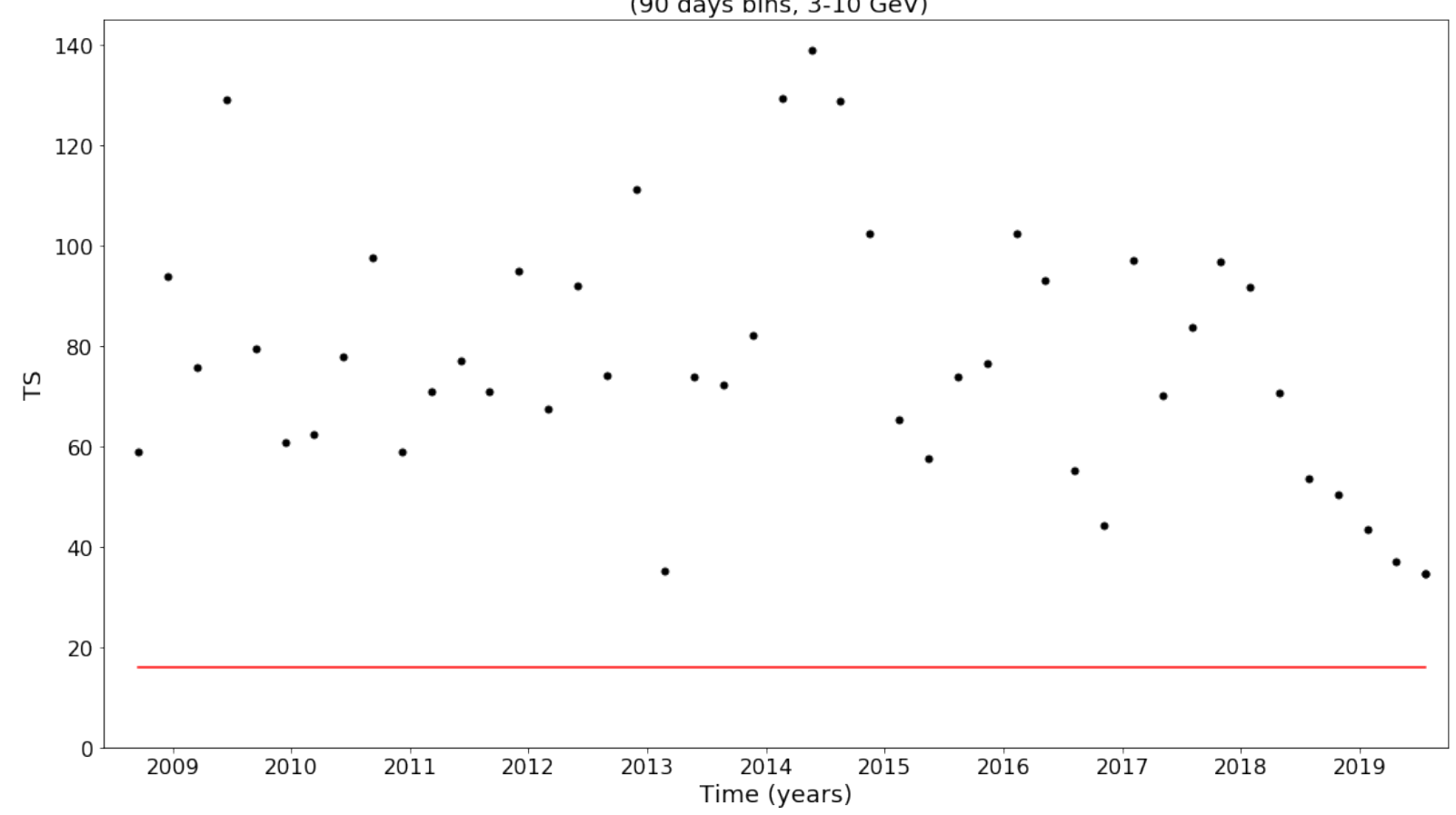

Figure M.3: The TS of the 90 days LC of 4FGL J1745.6-2859 in the 3-10 GeV energy band. The red line indicate the limit of $T S=16$. 
TS

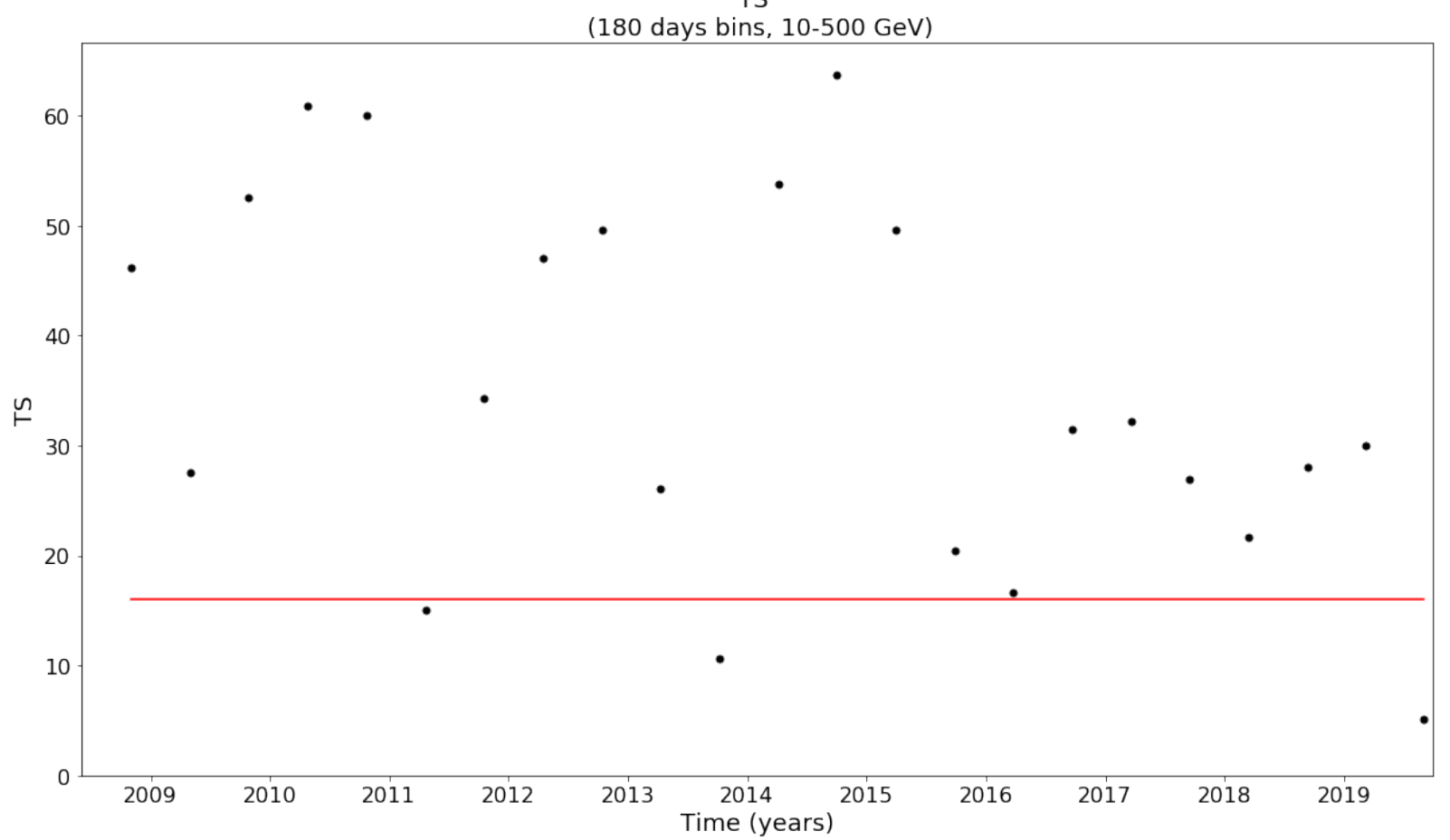

Figure M.4: The TS of the 180 days LC of 4FGL J1745.6-2859 in the 10-500 GeV energy band. The red line indicate the limit of $T S=16$.

TS

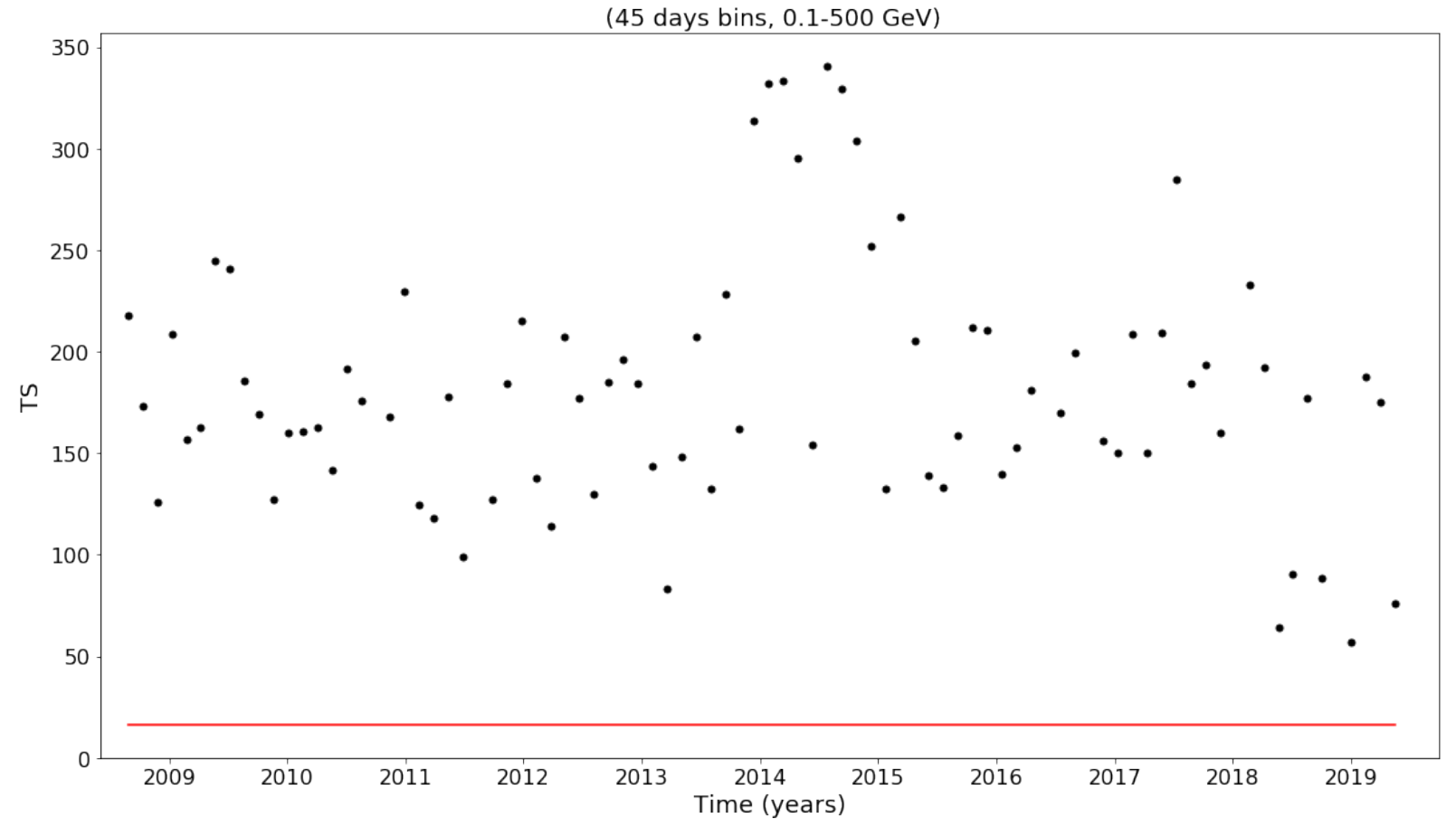

Figure M.5: The TS of the 45 days LC of 4FGL J1745.6-2859 in the $100 \mathrm{MeV}-500 \mathrm{GeV}$ energy band. The red line indicate the limit of $T S=16$. 